\title{
FY-2011 Status Report for Thermodynamics and Kinetics of Advanced Separations Systems
}

Leigh R. Martin

Peter R. Zalupski

Travis S. Grimes

September 2011

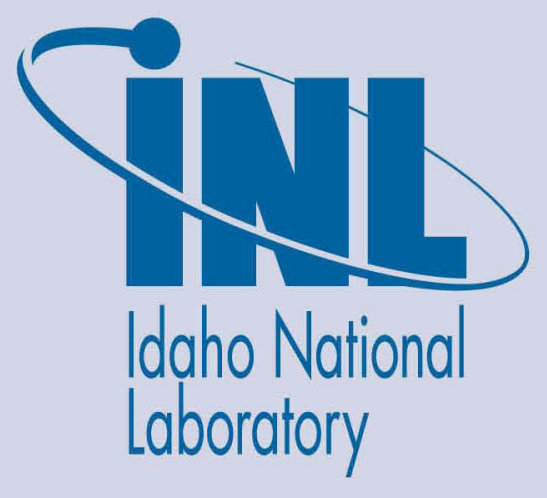

The INL is a U.S. Department of Energy National Laboratory operated by Battelle Energy Alliance 
INL/EXT-11-23681

FCRD-SWF-2011-000367

\title{
FY-2011 Status Report for Thermodynamics and Kinetics of Advanced Separations Systems
}

\author{
Leigh R. Martin \\ Peter R. Zalupski \\ Travis S. Grimes
}

September 2011

\section{Idaho National Laboratory \\ Fuel Cycle Research \& Development Idaho Falls, Idaho 83415}

http://www.inl.gov

Prepared for the

U.S. Department of Energy

Office of Nuclear Energy

Under DOE Idaho Operations Office

Contract DE-AC07-05ID14517 


\section{DISCLAIMER}

This information was prepared as an account of work sponsored by an agency of the U.S. Government. Neither the U.S. Government nor any agency thereof, nor any of their employees, makes any warranty,

expressed or implied, or assumes any legal liability or responsibility for the accuracy, completeness, or usefulness, of any information, apparatus, product, or process disclosed, or represents that its use would not infringe privately owned rights. References herein to any specific commercial product, process, or service by trade name, trade mark, manufacturer, or otherwise, does not necessarily constitute or imply its endorsement, recommendation, or favoring by the U.S. Government or any agency thereof. The views and opinions of authors expressed herein do not necessarily state or reflect those of the U.S. Government or any agency thereof. 


\section{SUMMARY}

This report presents a summary of the work performed in the area of thermodynamics and kinetics of advanced separations systems under the Fuel Cycle Research and Development (FCR\&D) program during FY 2011 at the INL.

On the thermodynamic front, investigations of liquid-liquid distribution of lanthanides at TALSPEAK-related conditions continued in FY11. It has been determined that a classical ion-exchanging phase transfer mechanism, where three HDEHP dimers solvate the metal ion in the organic phase, dominates metal extraction for systems that contain up to $0.1 \mathrm{M}$ free lactate in solution. The correct graphical interpretation of the observed data in those regions relied on incorporating corrections for nonideal behavior of HDEHP dimer in aliphatic diluents as well as sodium extraction equilibria. When aqueous conditions enter the complex regions of high lactate concentrations, slope analysis is no longer possible. When normalized metal distribution ratios were studied along the increasing concentration of free lactate, a slope of -1 was apparent. Such dependency either indicates aqueous complexing competition from lactate, or, a more likely scenario, a participation of lactate in the extracted metal complex. This finding agrees with our initial assessment of postulated changes in the extraction mechanism as a source of the lactate-mediated loss of extraction efficiency. The observed shape in the lanthanide distribution curve in our studies of TALSPEAK systems was the same for solutions containing no lactate or $2.3 \mathrm{M}$ lactate. As such we may conclude that the mechanism of phase transfer is not altered dramatically and remains similarly sensitive to effective charge density of the metal ion. In addition to these thermodynamics studies, this report also summarizes the first calorimetric determination of heat of extraction of ${ }^{248} \mathrm{Cm}$ in a bi-phasic system. The heat of extraction measured by isothermal titration calorimetry is compared to that determined using van't Hoff calculations.

Further investigations on the kinetics of extraction in TALSPEAK with macro quantities of lanthanides present in the initial aqueous phase composition have been performed. These results have been summarized and compared to previous work performed for FCR\&D. In addition, the effects of HDEHP concentration in the organic phase on europium extraction have been studied. 


\section{CONTENTS}

SUMMARY iv

ACRONYMS xii

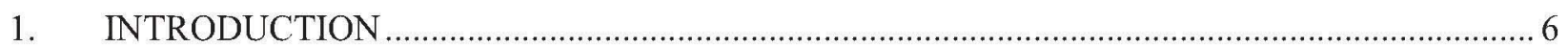

2. Results and Discussion ..................................................................................................... 7

2.1 : Liquid-liquid Distribution of Lanthanides as Mediated by Lactate and HDEHP in TALSPEAK Solutions

2.2 Studies on the Thermodynamic Balance of the TALSPEAK Distribution System.

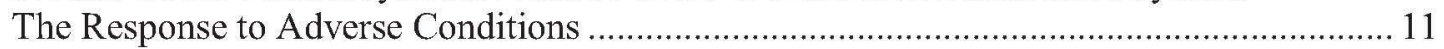

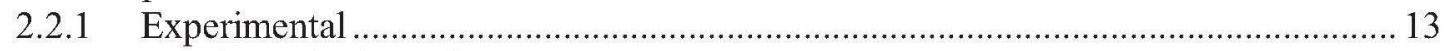

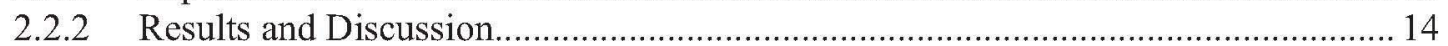

2.3 First Calorimetric Determination of Heat of Extraction of ${ }^{248} \mathrm{Cm}$ in a Bi-phasic

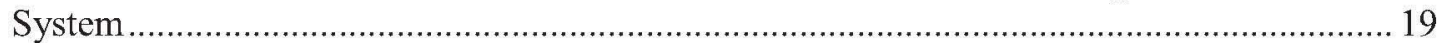

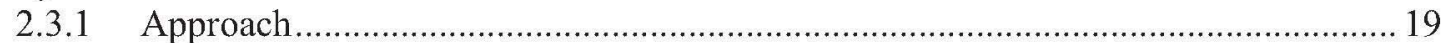

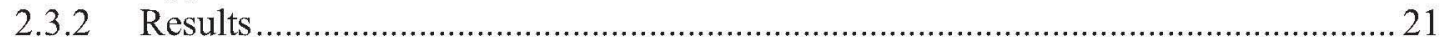

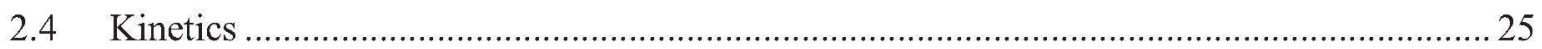

2.4.1 Aqueous Phase Complexation Kinetics .................................................................... 25

2.4.2 TALSPEAK Solvent Extraction Kinetics ............................................................. 28

2.4.3 Effect of HDEHP Concentration on Eu Solvent Extraction Kinetics ......................... 30

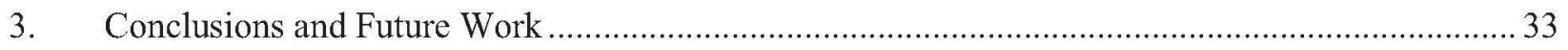

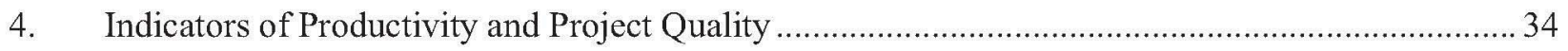

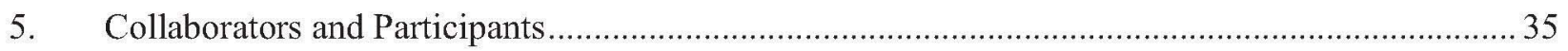

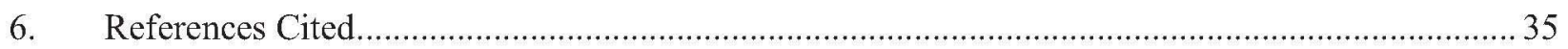

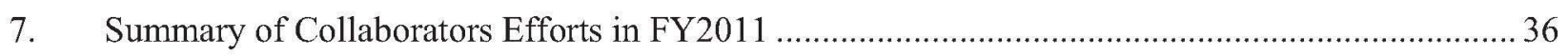

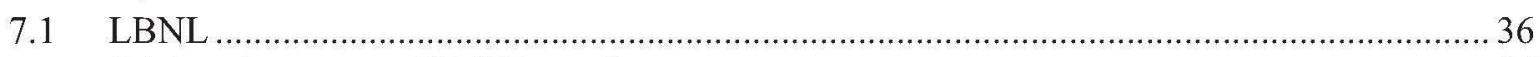

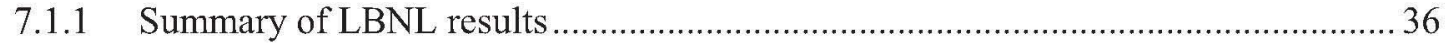

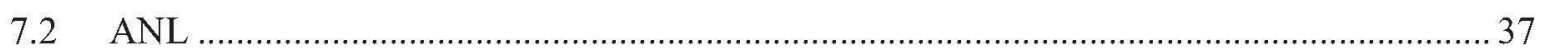

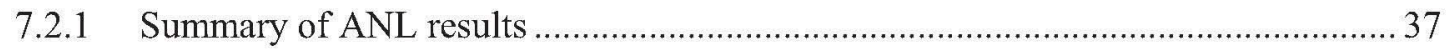

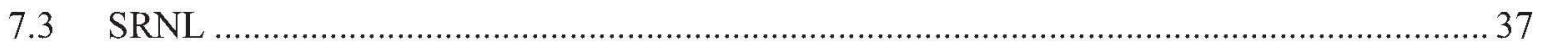

7.3.1 Electrochemical and Computation Investigation of TALSPEAK Chemistry …........ 37

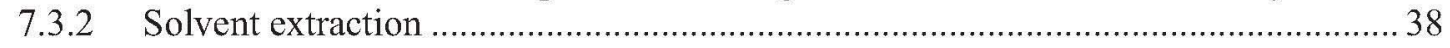

Appendix A Understanding Actinide/Lanthanide Speciation under TALSPEAK Conditions ................. 39

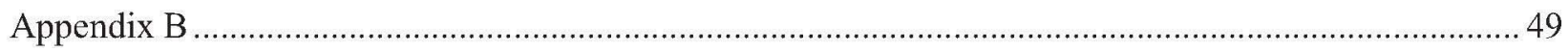

Separation Process Thermodynamics and Kinetics: Development of microfluídic devices for solvent extraction studies and radioanalytical applications .................... 49

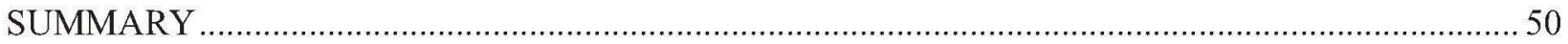




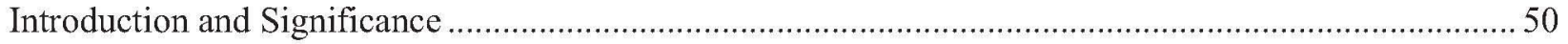

Comparison of Kinetics Experimental Methods ...................................................................... 50

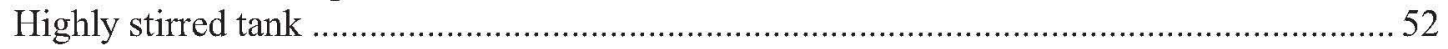

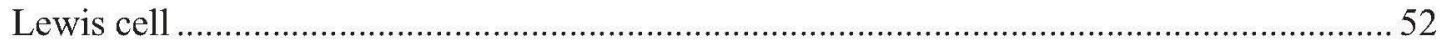

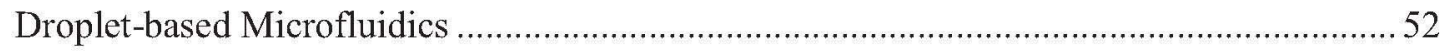

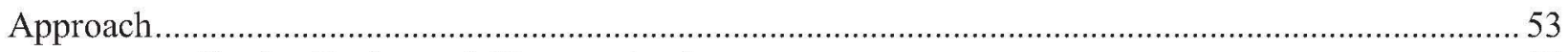

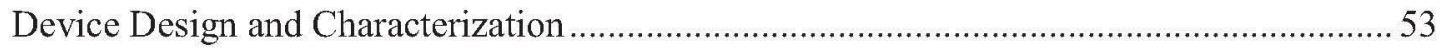

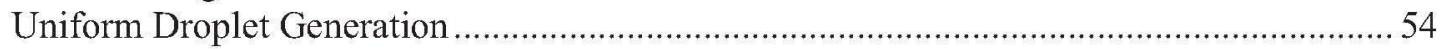

Rapid Mixing: Operation in the Kinetic Regime ............................................................ 55

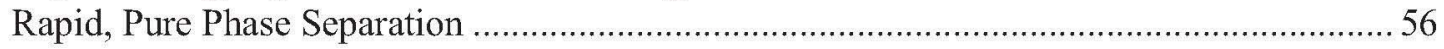

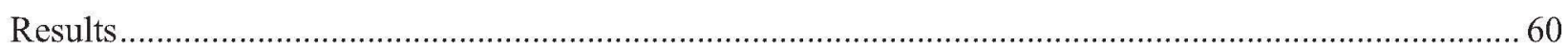

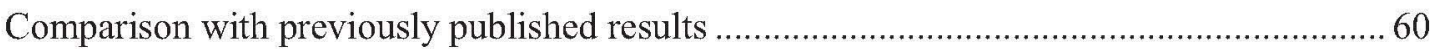

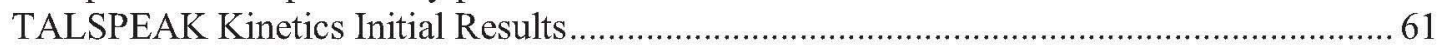

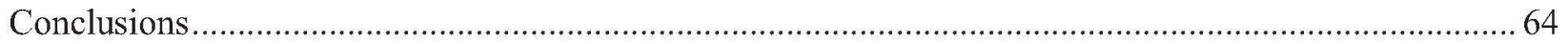

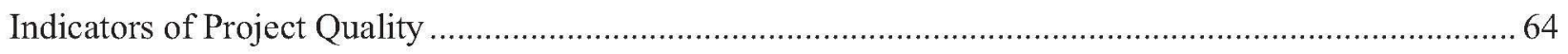

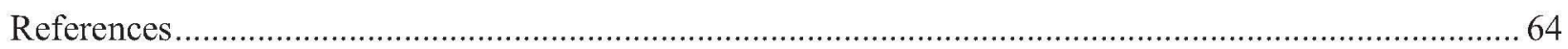

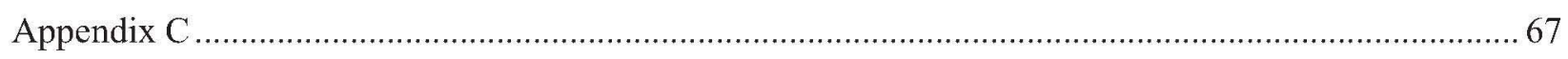

Electrochemical and Computation Investigation of the TALSPEAK Processes .....................................6 67

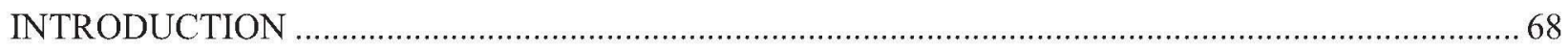

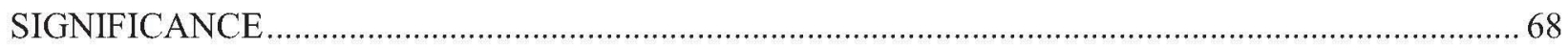

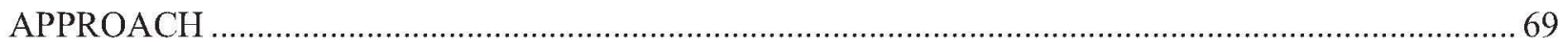

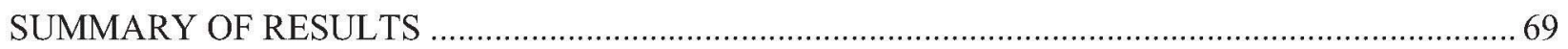

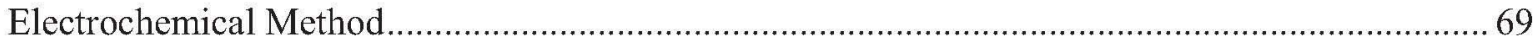

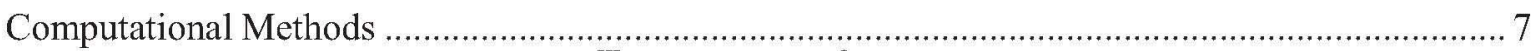

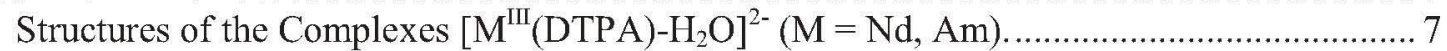

Calculated Change in Gibbs Free Energy for the Complexation of

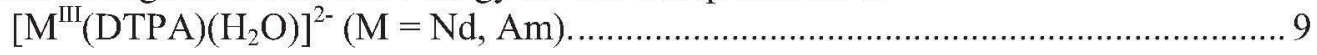

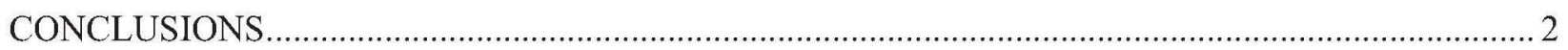

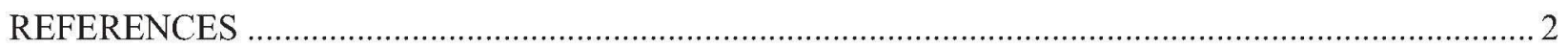

INDICATORS OF PROJECT QUALITY AND PRODUCTIVITY …............................................. 3

Appendix D Thermodynamic and Kinetic Studies of the TALSPEAK Solvent Extraction Process.......... 4

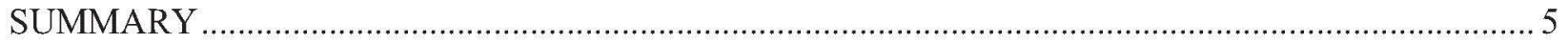




\section{FIGURES}

Figure 1. (a) Lactate dependency for the liquid-liquid distribution of $\mathrm{Ce}^{3+}$. Organic phase: HDEHP in n-dodecane. Aqueous phase: $\left[\mathrm{Ln}^{3+}\right]$ total $=1 \mathrm{mM}, \mathrm{p}\left[\mathrm{H}^{+}\right]=3.5,[\mathrm{DTPA}]=$ $0.05 \mathrm{M}$, varying $\left[\mathrm{Lac}^{-}\right]_{\mathrm{eq}}, \mathrm{I}=1-2 \mathrm{M}$ after $\mathrm{pH}$ adjustment. (b) The comparison of the extraction of lanthanide mixture $\left(\mathrm{La}^{3+}-\mathrm{Ho}^{3+}\right)$ by $0.2 \mathrm{M}$ HDEHP in n-dodecane from pH 3.5 aqueous solutions containing 0.05 M DTPA, and either no lactate or $1.0 \mathrm{M}$ free lactate at equilibrium.

Figure 2. (a) Extractant dependencies for the extraction of $\mathrm{Eu}^{3+}$ from TALSPEAK mixtures containing 0,0.5 and $1.0 \mathrm{M}$ free lactate at equilibrium. (b) Liquid-liquid distribution of $\mathrm{Eu}^{3+}$ from mineral acid solutions before and after the correction for the non-ideal solution behavior of HDEHP dimer in n-dodecane. (c) HDEHP dimer activity correction for the extractant dependency for the extraction of $\mathrm{Eu}^{3+}$ from TALSPEAK mixtures containing no lactate in solution.

Figure 3 (a) Extractant dependencies for the extraction of $\mathrm{Eu}^{3+}$ from TALSPEAK mixtures containing 0 and $1.0 \mathrm{M}$ free lactate at equilibrium using HDEHP in $n$-dodecane. (b) Extractant dependencies for the extraction of $\mathrm{Eu}^{3+}$ from TALSPEAK mixtures containing 0 and $1.0 \mathrm{M}$ free lactate at equilibrium using HDEHP in DIPB. (c) Sodium extraction corrected effective extractant dependencies for the extraction of $\mathrm{Eu}^{3+}$ from TALSPEAK mixtures containing no lactate in solution.

Figure 4 (a) Combined lactate dependencies for the liquid-liquid distribution of $\mathrm{Eu}^{3+}$. Organic phase: $0.1 \mathrm{M}$ HDEHP in $n$-dodecane. Aqueous phase: $\left[\mathrm{Ln}^{3+}\right]$ total $=1 \mathrm{mM}, \mathrm{p}\left[\mathrm{H}^{+}\right]=3.5$, $[\mathrm{DTPA}]=0.05 \mathrm{M}$, varying $\left[\mathrm{Lac}^{-}\right]_{\mathrm{eq}}, \mathrm{I}=1-2 \mathrm{M}$ after $\mathrm{pH}$ adjustment. (b) Effect of free lactate on the distribution $\left(\log \mathrm{D} / \mathrm{D}^{\circ}\right)$ of $\mathrm{Eu}^{3+}$ calculated for 2 of 3 collected datasets that did not fall drastically to negative logarithms of metal distribution.

Figure 5. Structures of key components of TALSPEAK-type actinide/lanthanide separation, i.e. diethylenetriamine-N,N,N',N",N"'-pentaacetic acid and di(2-ethylhexyl)phosphoric acid.

Figure 6 (a) Opposing thermodynamic forces in the TALSPEAK separation as quantified for lanthanide elements by extraction constants (HDEHP) and complex dissociation constants (DTPA), plotted as a function of reverse ionic radius. For extraction constants, org: HDEHP in n-dodecane, aq: $1.0 \mathrm{M}\left(\mathrm{H}^{+}, \mathrm{Na}^{+}\right) \mathrm{ClO}_{4}$. Dissociation constants were taken from NIST database. (b) Final liquid-liquid distribution of actinides and lanthanides - a result of the thermodynamic struggle for balance between DTPA and HDEHP.

Figure 7. Distribution of Am(III), Ce(III) and Eu(III) for DTPA-containing TALSPEAK-type liquid-liquid systems. The distribution curve for lanthanide ions $(-)$ was reconstructed based on $\mathrm{D}_{\mathrm{Ce}}$ and $\mathrm{D}_{\mathrm{Eu}}$. Org: 0.1 M HDEHP in $n$-dodecane. Aq: 0.05 M DTPA, $1.0 \mathrm{M}$ appropriate buffer adjusted to $\mathrm{pH}$ under investigation.

Figure 8. Structures of the aqueous phosphonic acid complexing agents investigated within TALSPEAK-type actinide/lanthanide separation framework, i.e. diethylenetriamineN,N,N',N",N"-pentaphosphonic acid, DTPP, and the ethylenediaminetetraphosphonic acid, EDTP

Figure 9. Distribution of Am(III), Ce(III) and Eu(III) for DTPP-containing TALSPEAK-type liquid-liquid systems. The distribution curve for lanthanide ions $(-)$ was reconstructed based on $\mathrm{D}_{\mathrm{Ce}}$ and $\mathrm{D}_{\mathrm{Eu}}$. Org: 0.1 M HDEHP in $n$-dodecane. Aq: $0.05 \mathrm{M}$ DTPA, $1.0 \mathrm{M}$ appropriate buffer adjusted to $\mathrm{pH}$ under investigation. 
Figure 10. Distribution of Am(III), Ce(III) and Eu(III) for EDTP-containing TALSPEAK-type liquid-liquid systems. The distribution curve for lanthanide ions (-) was reconstructed based on $\mathrm{D}_{\mathrm{Ce}}$ and $\mathrm{D}_{\mathrm{Eu}}$. Org: 0.1 M HDEHP in $n$-dodecane. Aq: $0.05 \mathrm{M}$ DTPA, $1.0 \mathrm{M}$ appropriate buffer adjusted to $\mathrm{pH}$ under investigation.

Figure 11. The comparison of distribution ratios for $\mathrm{Am}$ (III), $\mathrm{Ce}(\mathrm{III})$ and $\mathrm{Eu}(\mathrm{III})$ collected at normal aqueous TALSPEAK conditions (0.05 M DTPA, 1.0 $\left.\mathrm{M} \mathrm{H}^{+} / \mathrm{Na}^{+}, \mathrm{Lac}^{-}, \mathrm{pH} 3.5\right)$, where an organic diluent varies between aliphatic hydrocarbon, $n$-dodecane, and aliphatic alcohol, 1-dodecanol.

Figure 12. Schematic illustration of the calorimetric titration vessel, and calorimetric cup to scale.

Figure 13. Calorimetric power compensation trace collected for the liquid-liquid distribution of $\mathrm{Sm}(\mathrm{III})$. Aqueous phase: $\mathrm{pH} 3.0,\left[\mathrm{NaNO}_{3}\right]=1.0 \mathrm{M},[\mathrm{Sm}(\mathrm{III})]=5 \mathrm{mM}$. Organic phase: $0.2 \mathrm{M}$ HDEHP in $n$-dodecane, titrant injection size $=20 \mu \mathrm{L}$.

Figure 14. Calorimetric power compensation trace collected for the liquid-liquid distribution of $\mathrm{Cm}(\mathrm{III})$. Aqueous phase: $\mathrm{pH} 3.0,\left[\mathrm{NaNO}_{3}\right]=1.0 \mathrm{M},[\mathrm{Cm}(\mathrm{III})]=10.55 \mathrm{mM}$. Organic phase: $0.2 \mathrm{M}$ HDEHP in $n$-dodecane, titrant injection size $=20 \mu \mathrm{L}$.

Figure 15. Calorimetric power compensation trace collected for the liquid-liquid distribution of $\mathrm{Cm}(\mathrm{III})$. Aqueous phase: $\mathrm{pH} 3.0,\left[\mathrm{NaNO}_{3}\right]=1.0 \mathrm{M},[\mathrm{Cm}(\mathrm{III})]=10.55 \mathrm{mM}$. Organic phase: 0.2 M HDEHP in $n$-dodecane, titrant injection size $=10 \mu \mathrm{L}$.

Figure 17. Liquid-liquid distribution of tracer $\mathrm{Cm}$ (III) as a function of temperature. Aqueous phase: $\mathrm{pH} 1.58,\left[\mathrm{NaNO}_{3}\right]=1.0 \mathrm{M},[\mathrm{Cm}(\mathrm{III})]=$ tracerM . Organic phase: $0.2 \mathrm{M}$ HDEHP in $n$-dodecane.

Figure 19 UV-visible spectra of a) bound and unbound EBT $[\mathrm{Eu}]=1.0 \times 10^{-5} \mathrm{M},[\mathrm{EBT}]=1 \times 10^{-5}$ $\mathrm{M}$, and $\mathrm{b}$ ) bound and unbound $\mathrm{XO}[\mathrm{Eu}]=1 \times 10^{-5} \mathrm{M},[\mathrm{XO}]=1 \times 10^{-5} \mathrm{M}, \ldots$

Figure 20. Sample kinetics trace from the stopped flow spectrophotometer measuring the rate of reaction of Eu-EBT complex with DTPA. $[\mathrm{Eu}]=5 \times 10^{-6} \mathrm{M},[\mathrm{EBT}]=1 \times 10^{-5} \mathrm{M}$, $[$ DTPA $]=1.25 \times 10^{-3} \mathrm{M}, \mathrm{pH} 3.6$.

Figure 22. Influence of [DTPA $]_{(\mathrm{aq})}$ on rate of extraction of cerium by $0.17 \mathrm{M}$ HDEHP in dodecane using a rotary mixer to agitate the two phases. Aqueous phase composition 1 $\mathrm{M}$ lactic acid at pH 3.8, [DTPA] 10-50 mM, [Ce] $10 \mathrm{mM}$.

Figure 23. Influence of [DTPA $]_{(\mathrm{aq})}$ on rate of extraction of tracer Eu by $0.17 \mathrm{M}$ HDEHP in dodecane using a rotary mixer to agitate the two phases. Aqueous phase composition $1.0 \mathrm{M}$ lactic acid at $\mathrm{pH} 3.8$, [DTPA] $10-50 \mathrm{mM}$, [Ce] $10 \mathrm{mM}$.

Figure 24. Influence of [DTPA $]_{(\mathrm{aq})}$ on rate of extraction of cerium by $0.17 \mathrm{M}$ HDEHP in dodecane using a rotary mixer to agitate the two phases. Aqueous phase composition 1 $\mathrm{M}$ lactic acid at pH 3.8, [DTPA] 10-50 mM, [Ce] $10 \mathrm{mM}$, [Eu] $10 \mathrm{mM}$.

Figure 25. Influence of [DTPA $]_{(\mathrm{aq})}$ on rate of extraction of tracer Eu by $0.17 \mathrm{M}$ HDEHP in dodecane using a rotary mixer to agitate the two phases. Aqueous phase composition 1.0 M lactic acid at pH 3.8, [DTPA] $10-50 \mathrm{mM},[\mathrm{Ce}] 10 \mathrm{mM},[\mathrm{Eu}] 10 \mathrm{mM}$.

Figure 26. Influence of [HDEHP] $]_{(\text {org })}$ on rate of extraction of Eu using a vortex mixer to agitate the two phases. Aqueous phase composition $1 \mathrm{M}$ lactic acid at $\mathrm{pH} 3.6,[\mathrm{DTPA}]=$ $50 \mathrm{mM}$. 
Figure27. Plots of distribution ratio as a function of Equation 11 vs time for HDEHP concentrations of a) $0.2 \mathrm{M}$, b) $0.5 \mathrm{M}$ and c) $1.0 \mathrm{M}$. Aqueous phase composition $1 \mathrm{M}$ lactic acid at $\mathrm{pH} 3.6,[\mathrm{DTPA}]=50 \mathrm{mM}$.

Figure 2. Device design and operation to measure rate constants of interfacial mass transfer in solvent extraction. (top) A schematic shows the design of the device. A single piece of Teflon ${ }^{\circledR}$ FEP tubing was micromachined to contain a droplet generator (i), a channel where the extraction occurs (reaction channel), and a phase separator (ii). One syringe pump (left, gray box) is used to control the inflow rates, and a second syringe pump (right, gray box) is used to set the rate of outflow of separated organic phase. The aqueous outflow is collected in aliquots for offline analysis. Additional Swagelok hardware (not shown above but detailed in SI) is utilized to properly seal the multiple pieces of tubing, and direct the output capillaries from the syringe pump (bottom) Photos of the regions in the schematic outlined by black dashes show the micromachined holes in the inner FEP tubing: one set of five holes for the droplet generator (i) and two parallel sets of five holes for the phase separator (ii). 


\section{TABLES}

Table 1. Heats of extraction and errors determined for samarium and curium in the biphasic

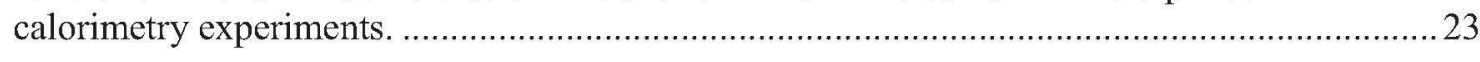

Table 2. Average kinetic parameters determined for the reaction of DTPA with Eu ............................28

Table 3. Calculated kinetic parameters for Eu extraction at various HDEHP concentrations in the organic phase 


\section{ACRONYMS}

\begin{tabular}{|c|c|}
\hline EBT & Eriochrome Black T (EBT) \\
\hline $\mathrm{D}$ & Distribution coefficient \\
\hline DIAMEX & DIAMide EXtraction \\
\hline DIBP & 1,4-diisopropylbenzene \\
\hline DTPA & Di 2-ethylenetriamine-N,N,N',N",N"'-pentaacetic acid \\
\hline FCR\&D & Fuel Cycle Research and Development \\
\hline HDEHP & Di ethyl hexyl phosphoric acid \\
\hline ICPMS & Inductively Coupled Plasma Mass Spectrometry \\
\hline INL & Idaho National Laboratory \\
\hline MOX & Mixed Oxide Fuel \\
\hline ORNL & Oak Ridge National Laboratory \\
\hline PUREX & Plutonium Uranium Reduction EXtraction \\
\hline SANEX & Selective ActiNide EXtraction \\
\hline TALSPEAK & $\begin{array}{l}\text { Trivalent Actinide Lanthanide Separations by Phosphorus-reagent Extraction } \\
\text { from Aqueous Komplexes }\end{array}$ \\
\hline US DOE & United States Department of Energy \\
\hline UV-vis & Ultra Violet-visible \\
\hline WSU & Washington State University \\
\hline $\mathrm{XO}$ & Xylenol orange \\
\hline
\end{tabular}




\section{SEPARATIONS CAMPAIGN}

\section{FY-2011 STATUS REPORT FOR THERMODYNAMICS AND KINETICS OF ADVANCED SEPARATIONS SYSTEMS}

\section{INTRODUCTION}

The distribution of a solute between two immiscible solvents is one of the most important technologies for the separations industry. Liquid-liquid distributions have long been used for purification of chemicals in a wide variety of industrial applications from pharmaceuticals to industrial waste treatment.[1] To successfully develop and understand new separations systems, the role of thermodynamics and kinetics in all of the chemical processes involved in both the aqueous and organic phases needs to be clearly understood. Even for simple systems this can be an expansive task. When applying this to solvent extraction processes for the nuclear industry, it becomes significantly more challenging as reliable fundamental data on the behavior of actinides in solution are not widely available.

If the US is to develop a sustainable closed nuclear fuel cycle, significant developments in the area of fuel utilization and reducing the radiotoxicity in the final waste-forms will have to be made. To reduce the radiotoxicity in the final wasteforms, some level of used fuel processing will be required to remove the minor actinides for re-use or burning in a reactor. There are currently two main philosophies for the recycle of the minor actinides using solvent extraction. The first is the use of ligands that contain soft donor atoms (such as $\mathrm{N}$ or $\mathrm{S}$ ) for the group separation of the trivalent actinides and lanthanides. Examples of this group separation strategy exist in the DIAMEX-SANEX[2-4] process under development in Europe and TRUEX-TALSPEAK[5-7] in the US. The second approach utilizes the higher oxidation states of americium for its selective separation from curium and the lanthanides.[8]

Regardless of the approach taken, both the thermodynamic driving forces behind these separations $(\Delta \mathrm{G}, \Delta \mathrm{H}$ and $\Delta \mathrm{S}$ ) and the kinetics of extraction would have to be thoroughly understood before any process could be implemented even at a pilot scale. The aim of the Thermodynamics and Kinetics research program as a whole is to not only grow expertise in actinide solution chemistry, but to perform fundamental chemical measurements on actinide bearing systems so that any actinide separations system can be understood at a fundamental level. By concentrating on the thermodynamic and kinetic features of a chemical separations process it is envisaged that more accurate models for any process can be built.

This report documents the research performed at the INL in FY 2011 that supports the Thermodynamics and Kinetics of Advanced Separations Systems research program. The techniques that have been employed this year include traditional solvent extraction studies for both thermodynamic and kinetic studies, isothermal titration calorimetry and stopped flow spectrophotometry. These techniques have been utilized to expand the understanding of curium extraction thermodynamics and lanthanide extraction kinetics in TALSPEAK solvent extraction systems. In addition a brief summary of the research efforts at LBNL, ANL and SRNL conducted under the Thermodynamics and Kinetics of Advanced Separations Systems are presented. 


\section{Results and Discussion}

\subsection{Liquid-liquid Distribution of Lanthanides as Mediated by Lactate and HDEHP in TALSPEAK Solutions}

FY11 investigations into the effects of increasing complexity of the organic phase on the fundamental aspects of TALSPEAK chemistry focused on studying two problematic observations that developed during initial work. The reader is referred to the FY10 report for the detailed description of the collected liquid-liquid distribution studies that served as a platform for current investigations presented in this report [9]. In summary, the distribution of lanthanide metal ions between aqueous systems of varying total lactate concentration and constant $\mathrm{pH}$ and organic systems of varying concentration of liquid cation exchanger - HDEHP - was monitored. The results for the distribution of cerium are summarized in Figure 1 and represent trends observed for all investigated lanthanides $\left(\mathrm{La}^{3+}-\mathrm{Ho}^{3+}\right)$.
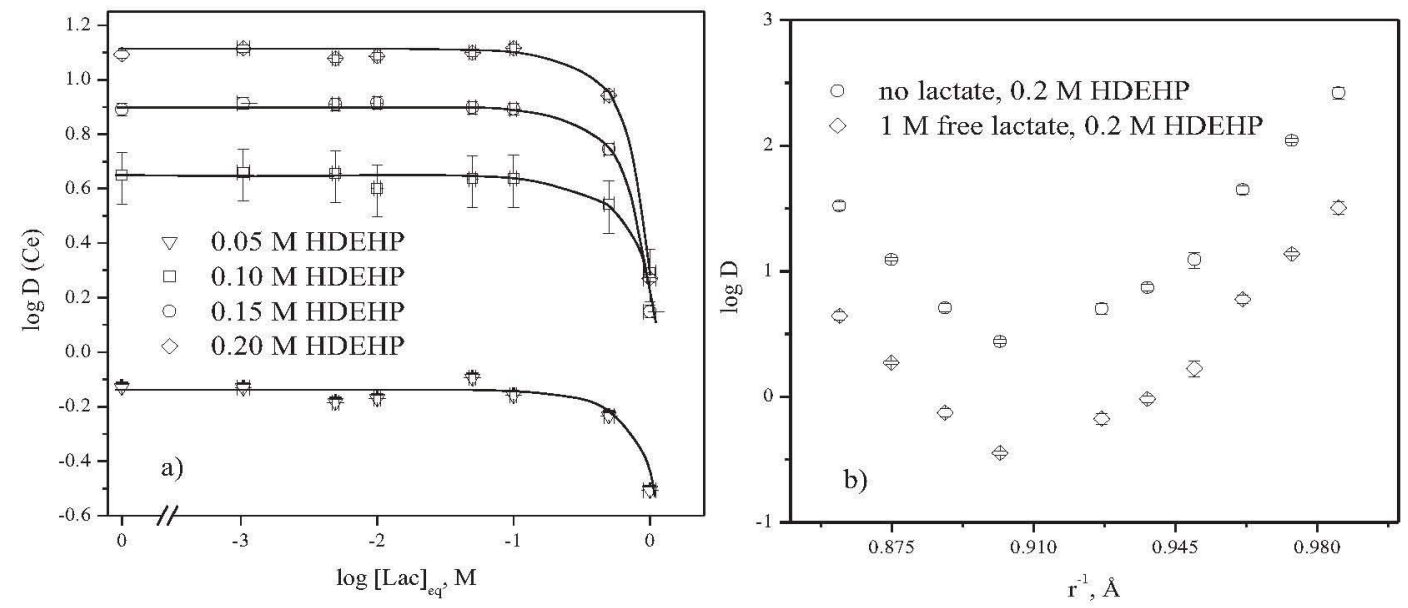

Figure 1. (a) Lactate dependency for the liquid-liquid distribution of $\mathrm{Ce}^{3+}$. Organic phase: HDEHP in $\mathrm{n}^{-}$ dodecane. Aqueous phase: $\left[\mathrm{Ln}^{3+}\right]$ total $=1 \mathrm{mM}, \mathrm{p}\left[\mathrm{H}^{+}\right]=3.5,[\mathrm{DTPA}]=0.05 \mathrm{M}$, varying $\left[\mathrm{Lac}^{-}\right]_{\mathrm{eq}}, \mathrm{I}=1-2$ $\mathrm{M}$ after $\mathrm{pH}$ adjustment. (b) The comparison of the extraction of lanthanide mixture $\left(\mathrm{La}^{3+}-\mathrm{Ho}^{3+}\right)$ by $0.2 \mathrm{M}$ HDEHP in n-dodecane from pH 3.5 aqueous solutions containing $0.05 \mathrm{M}$ DTPA, and either no lactate or $1.0 \mathrm{M}$ free lactate at equilibrium.

The distribution of lanthanide ions follows a characteristic trend across the investigated range of aqueous lactate concentrations. The initial flat dependency is indicative of lactate's inertness in the system where the liquid-liquid distribution of metal ions is dictated by a thermodynamic balance between a phase transfer reagent - HDEHP - and an aqueous polyamioncarboxylate complexing reagent - DTPA. The distribution ratios are independent of lactate up to $0.1 \mathrm{M}$ concentration. As the concentration of free lactate enters those complex buffer regions, corresponding with the operational conditions of TALSPEAK, the distribution ratios decrease despite constant $\mathrm{pH}$ of the aqueous phase. This characteristic dip in the metal ion partitioning was observed across the entire investigated series of lanthanides, suggesting that the mechanism of phase transfer must be related to that of usual HDEHP-mediated cation exchange. A change in the stoichiometry of the extracted complex has to be considered, however, when seeking origins of the diminished extraction.

The FY11 research activities targeted the observed complexities summarized above. The chemistry of TALSPEAK-type separation was monitored by exploring several system variables. While the variation of the concentration of HDEHP played a central role in all studies the effects of growing lactate 
concentration, the nature of the carboxylate buffer and diluent effects were all explored. Here those investigations are sequentially discussed with an overall intent to gain further understanding of the complexities of the TALSPEAK process.
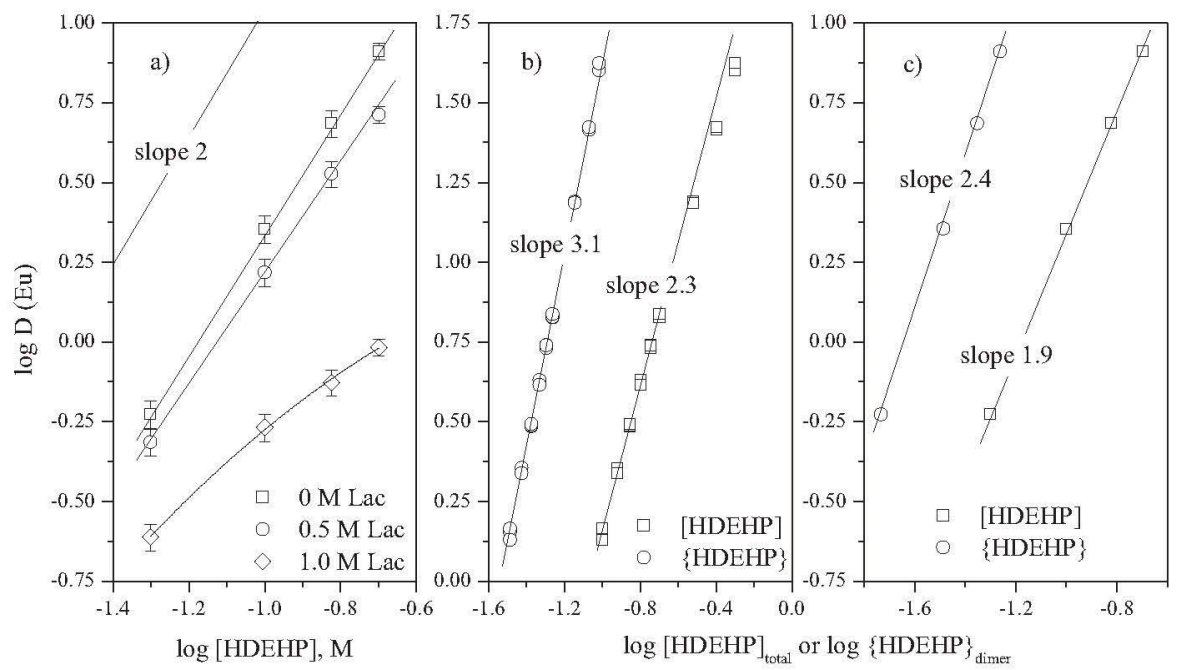

Figure 2. (a) Extractant dependencies for the extraction of $\mathrm{Eu}^{3+}$ from TALSPEAK mixtures containing $0,0.5$ and $1.0 \mathrm{M}$ free lactate at equilibrium. (b) Liquid-liquid distribution of $\mathrm{Eu}^{3+}$ from mineral acid solutions before and after the correction for the non-ideal solution behavior of HDEHP dimer in $\mathrm{n}$-dodecane. (c) HDEHP dimer activity correction for the extractant dependency for the extraction of $\mathrm{Eu}^{3+}$ from TALSPEAK mixtures containing no lactate in solution.

Figure 2 summarizes the slope analyses for the selected europium distribution data as a function of HDEHP in $n$-dodecane. The observed slope of 2 for the extractant dependency (Figure 2a) persists for the initial six aqueous conditions where free lactate concentrations range from 0 to $0.1 \mathrm{M}$. The slope is suppressed further for the final two conditions, where 0.5 and $1.0 \mathrm{M}$ free lactate is present. Based on the expected stoichiometry of the phase transfer reaction, where a trivalent cation is extracted by a cation exchanger, a slope of 3 is expected from the distribution ratio data presented as a function of extractant concentration. A slope suppression for this type of data is common, however, and corresponds with a nonideal behavior of the extractant dimer in aliphatic diluents such as n-dodecane. A dimer activity correction, developed by Danesi and Vandegrift, accounts for this observed non-ideal chemistry, allowing unambiguous assignment of the stoichiometry of extraction [10]. Figure $2 \mathrm{~b}$ illustrates the correction for the extraction of europium from the mineral acid solutions by HDEHP in $n$-dodecane. Interestingly, when a similar exercise was applied to the collected TALSPEAK-type liquid-liquid systems, a problematic slope of 2.4 resulted as illustrated by Figure $2 \mathrm{c}$. When lactate buffer was replaced by a malonate complexing medium no differences in slope behavior were observed, suggesting that an equilibrium unassociated with the buffering agent may be responsible for this slope suppression. To further examine this issue the effects of the diluents were investigated. Liquid-liquid distribution of europium between aqueous mixtures of $0,0.1$ and $1.0 \mathrm{M}$ free lactate and $50 \mathrm{mM}$ DTPA and organic solutions of $0.1,0.2$ and 0.3 M HDEHP in 1,4-diisopropylbenzene, (DIPB), were collected. No non-ideal interactions between HDEHP dimers are expected in aromatic diluents such as DIPB [11]. As such a suppression of the slope for the extractant dependency should be eliminated in these systems. Figure 3 compares the observed diluents effects. The distribution of europium uncorrected for activity effects is presented in Figure $3 \mathrm{a}$ for mixtures without lactate and $1 \mathrm{M}$ free lactate. Figure $3 \mathrm{~b}$ collects the matching extractant dependencies in DIPB. As expected, the slope for the extractant dependency for a no-lactate scenario (representing all dilute lactate conditions) in aromatic diluent was higher than the corresponding value collected in the 
aliphatic mixtures. In fact, the slope matches that of the $n$-dodecane system after the non-ideal chemistry has been accounted for. Our diluent study successfully considered the effects of the non-ideal behavior of HDEHP dimers on the interpretation of the liquid-liquid distribution data. To explain the remaining slope deviation in TALSPEAK-type mixtures it is necessary to compare those aqueous conditions with aqueous mixtures of the 2-phase distribution systems where the activity correction was successfully incorporated in the slope analyses. Such aqueous phases consist of moderate concentrations the mineral acid $(0.1-0.5$ M). TALSPEAK-type mixtures are characterized by a much lower acidity (1 mM). As such it is appropriate to examine the extraction of sodium, whose $2^{\text {nd }}$ power dependency on aqueous acid concentration is quite steep, as the contributing source of the slope suppression. The extraction of sodium could thus occupy a fraction of the extractant and influence the extraction of trivalent europium.
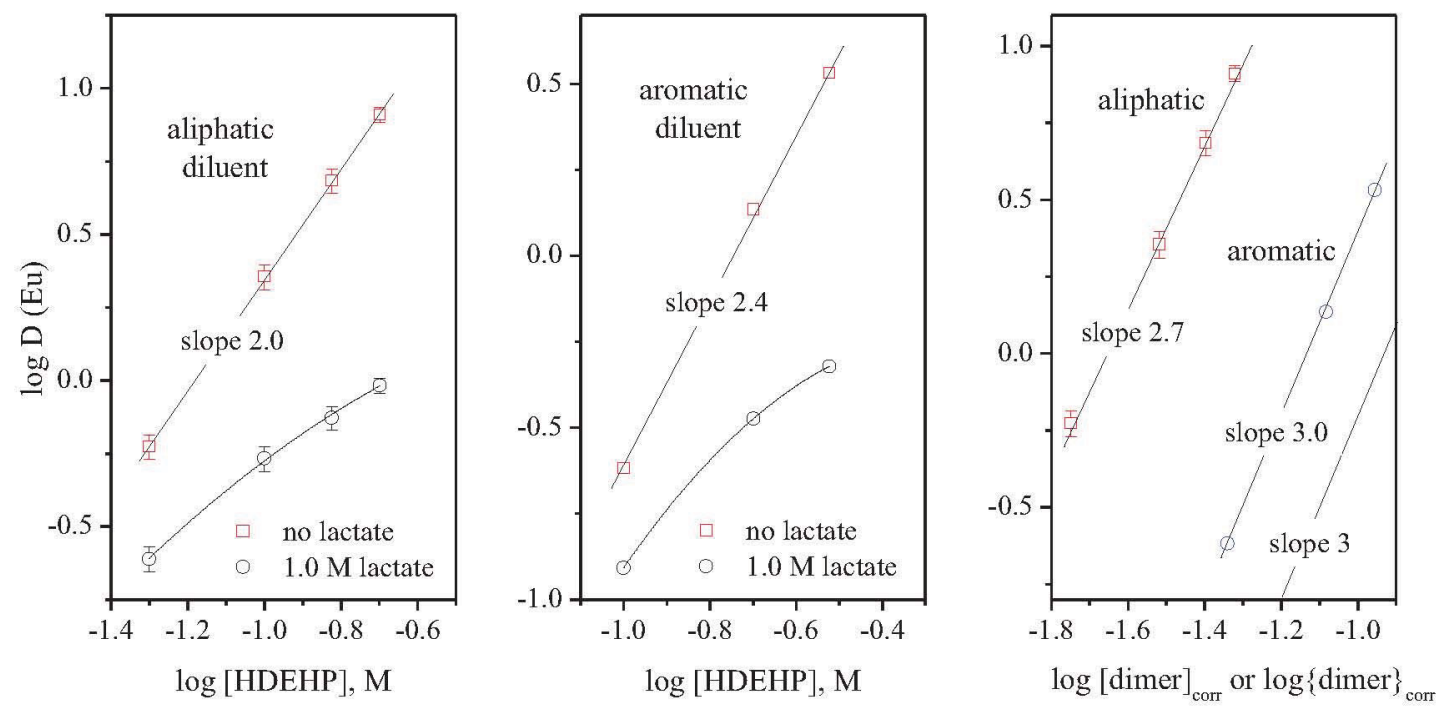

Figure 3 (a) Extractant dependencies for the extraction of $\mathrm{Eu}^{3+}$ from TALSPEAK mixtures containing 0 and $1.0 \mathrm{M}$ free lactate at equilibrium using HDEHP in $n$-dodecane. (b) Extractant dependencies for the extraction of $\mathrm{Eu}^{3+}$ from TALSPEAK mixtures containing 0 and $1.0 \mathrm{M}$ free lactate at equilibrium using HDEHP in DIPB. (c) Sodium extraction corrected effective extractant dependencies for the extraction of $\mathrm{Eu}^{3+}$ from TALSPEAK mixtures containing no lactate in solution.

The extraction equilibrium expression for liquid-liquid distribution of sodium is shown in Equation 1. The extraction constant used for the correction was reported by McDowell et.al. [12].

$$
K_{e x}=\frac{\left[N a L(H L)_{3}\right]\left[H^{+}\right]}{\left[N a^{+}\right]\left[(H L)_{2}\right]^{2}}, \log K_{e x}=-3.56
$$

Figure $3 \mathrm{c}$ shows the extractant dependencies for the distribution of europium after the calculation of the effective ligand concentration, i.e. consideration of sodium partitioning. Slopes for both diluent environments return to the expected third power dependencies, supporting a classical mechanism of extraction of trivalent metal ions in systems containing up to $\sim 0.3 \mathrm{M}$ total lactate. The phase transfer equilibrium can be written as:

$$
\operatorname{Ln}^{3+}+\overline{3(H A)_{2}} \Leftrightarrow \overline{\operatorname{Ln(HA_{2})_{3}}}+3 H^{+}
$$

Beyond those dilute buffering conditions, where total lactate concentration exceeds $0.5 \mathrm{M}$, the proper interpretation of the solvent extraction results using slope analysis methods is not possible. Those regions 
appear to be complicated by phase partitioning of lactate and water, along with the observed diminishing distribution of trivalent metal ions in a liquid-liquid system. Grimes et.al. studied the distribution of lactate and water at TALSPEAK-type conditions and concluded that the organic concentrations of both species significantly increase beyond the $0.5 \mathrm{M}$ total lactate content [13]. Similar conclusions may be drawn from our lactate dependency work. When 0.5 and $1.0 \mathrm{M}$ free lactate was present, the slopes for the extractant dependencies are suppressed further, which may be attributed to the observed decrease in the extraction efficiency at such conditions.
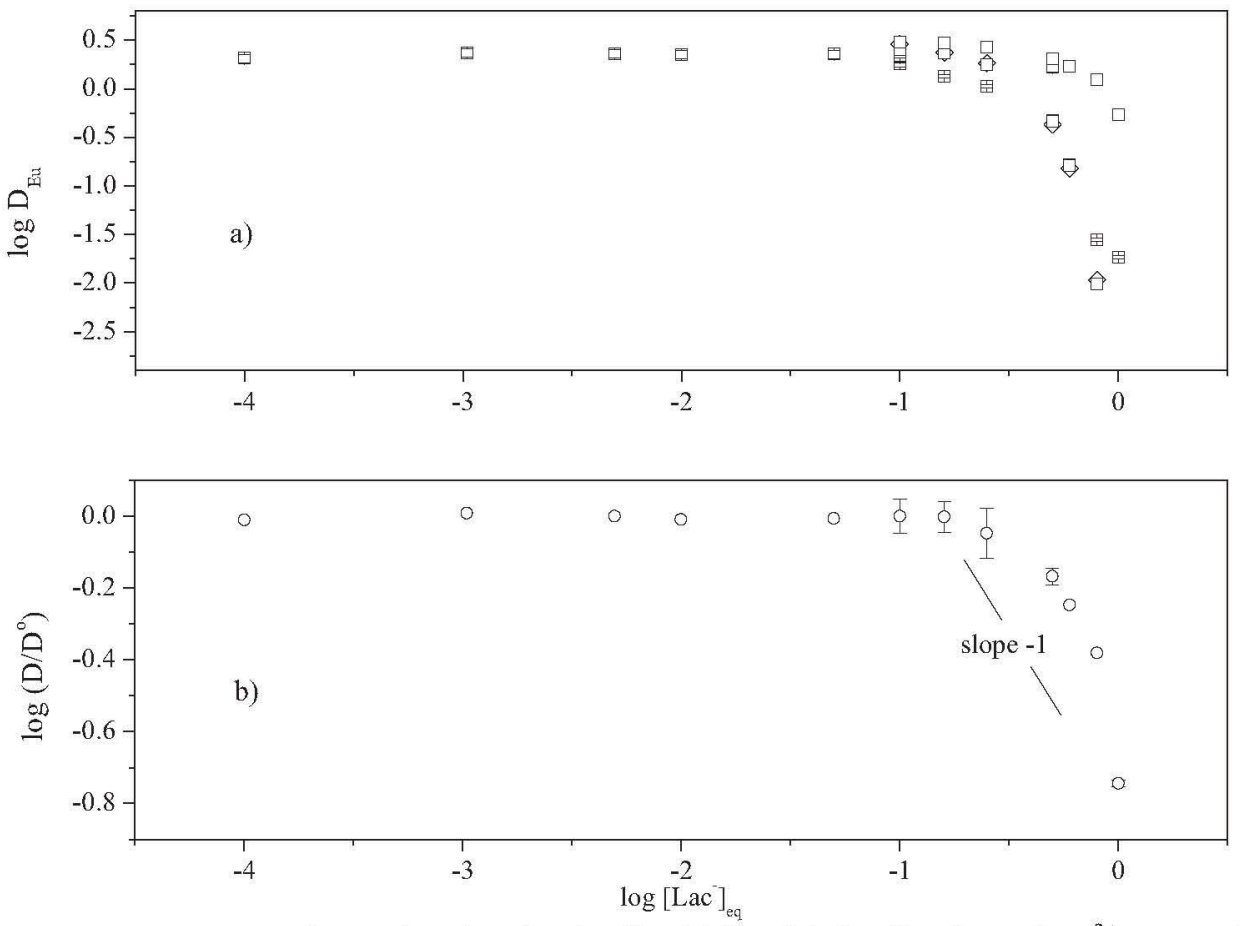

Figure 4 (a) Combined lactate dependencies for the liquid-liquid distribution of $\mathrm{Eu}^{3+}$. Organic phase: $0.1 \mathrm{M}$ HDEHP in $n$-dodecane. Aqueous phase: $\left[\mathrm{Ln}^{3+}\right]$ total $=1 \mathrm{mM}, \mathrm{p}\left[\mathrm{H}^{+}\right]=3.5$, [DTPA] $=0.05 \mathrm{M}$, varying $\left[\mathrm{Lac}^{-}\right]_{\mathrm{eq}}, \mathrm{I}=1-2 \mathrm{M}$ after $\mathrm{pH}$ adjustment. (b) Effect of free lactate on the distribution $\left(\log \mathrm{D} / \mathrm{D}^{\circ}\right)$ of $\mathrm{Eu}^{3+}$ calculated for 2 of 3 collected datasets that did not fall drastically to negative logarithms of metal distribution.

To look at this more closely we studied liquid-liquid distribution of europium in more detail in the region of high total lactate concentrations. Figure 4a summarizes the results of three independent studies on the europium extraction by $0.1 \mathrm{M}$ HDEHP in $n$-dodecane from TALSPEAK-type aqueous media, where $0.10,0.16,0.25,0.50,0.60,0.80$ and $1.0 \mathrm{M}$ aqueous free lactate concentrations were present. The characteristic descending trend in the metal ion distribution appears very unpredictable. Two out of three dependencies adopt a moderate trend of decreasing distribution ratios as lactate concentration is growing, relative to a very drastic fall of $\mathrm{D}$ values observed for one study. The origin of this discrepancy cannot be attributed to the metal ion concentration and remains unexplained. The collected distribution ratios for the two matching studies, when normalized using a $\mathrm{D} / \mathrm{D}^{\circ}$ analysis $\left(\mathrm{D}^{\circ}\right.$ is distribution of europium unaffected by the presence of lactate in solution), results in a direct relationship between the growing concentration of free lactate in solution and the descending trend of the europium distribution. Figure $4 \mathrm{~b}$ illustrates the linear relationship. The interpretation of the observed slope of -1 for this correlation summons two possibilities. The first possibility supports the existence of an aqueous complexation equilibrium capable of competing with the established HDEHP vs. DTPA thermodynamic balance. However, recent studies by Leggett and Jensen dispute the existence of any aqueous interference to the established thermodynamic 
balance that guides the actinide/lanthanide separations in the TALSPEAK process [14]. Their studies explored aqueous mixtures at $\mathrm{pH}$ conditions above 4, where the complexing power of DTPA is optimal. However, considering the currently accepted thermodynamic data, no reasonable theoretical calculation may support competitive complexation of trivalent metal ions by lactate in the presence of 0.05 M DTPA. The second possible explanation of the observed linear trend may indicate the participation of one lactate in the extracted metal complex. The negative dependency suggests that lactate participation decreases the overall thermodynamic stability of the complex, forming a less-polar shell around the metal ion and lowering the distribution ratios. The exact mechanism of the insertion of lactate into the metal complex remains uncertain. The metal may be extracted as a monolactate as postulated by Kosyakov and coauthors [15]. Lactate may also disrupt an HDEHP dimer and participate in the metal extraction as an aggregate. The studies by Grimes et.al. support this theory. They reported lactate partitioning data that does not stoichiometrically coincide with the mechanism of the extraction of the monolactate species, where a 1:1 metal/lactate concentration ratio would be expected in the organic phase.[13] In fact much greater concentrations of lactate exist in TALSPEAK-related non-aqueous mixtures.

No matter how lactate participates in the metal ion partitioning its behavior in TALSPEAK-type chemistry is problematic. Our studies clearly illustrate that a well-behaving 2-phase system, where metal ion partitioning may be described by a single equilibrium, may only be expected when dilute lactate conditions exist. TALSPEAK operational conditions venture into those unpredictable realms, where lactate tends to distribute in a liquid-liquid system, affecting the final destination of the metal ion. Since the mechanism(s) of lactate's behavior in TALSPEAK-type mixtures remain undefined the separation of actinides from lanthanides using this particular chemistry cannot be thermodynamically predicted when response to off-normal conditions is queried.

\subsection{Studies on the Thermodynamic Balance of the TALSPEAK Distribution System. The Response to Adverse Conditions}

With solvent extraction, the modification of the aqueous phase conditions to produce a holding-back influence that opposes the phase transfer process allows one to design of some of the most challenging of separations. An example of such a system positions an aqueous soft donor aminopolycarboxylate complexing agent, diethylenetriamine-N,N,N',N",N"'-pentaacetic acid, DTPA, against a powerful cation exchanger, di(2-ethylhexyl)phosphoric acid, HDEHP. Such is the backbone of the TALSPEAK process, a liquid-liquid distribution system designed to separate trivalent actinides from trivalent lanthanides [16]. Figure 5 shows the structures of the main components of the TALSPEAK process. The aqueous complexant preferentially holds the actinides in an aqueous lactate buffer solution, while the lanthanides are extracted into an organic phase by HDEHP. Weak covalent interactions between the soft Lewis base ligands such as DTPA and the actinides have been suggested as the source of the observed complexing preference over lanthanides [17]. Otherwise, $f$-element contraction renders the aqueous chemistries of $\mathrm{Am}$ (III) and $\mathrm{Cm}$ (III) equivalent to $\mathrm{Nd}$ (III), Pm(III) and $\mathrm{Sm}$ (III) when electrostatic interactions dominate the complexation by oxygen-donating ligands [18]. However, the actinide/lanthanide separation in this system is not accomplished solely due to the enhanced thermodynamic stability of DTPA $/ \mathrm{An}^{3+}$ complexes. Rather, it is the influence that the resultant product of the "DTPA vs. HDEHP battle" has on the lanthanide group extraction. The opposing complexing forces of DTPA and HDEHP illustrate a striking thermodynamic relationship, which, depending on the experimental conditions, favors either side and determines the final shape of the characteristic TALSPEAK lanthanide extraction curve. This relationship affects the U-shape of the curve similarly to a "see-saw" balancing act. Here we present several fundamental liquid-liquid distribution exercises that clearly illustrate this remarkable effect. 

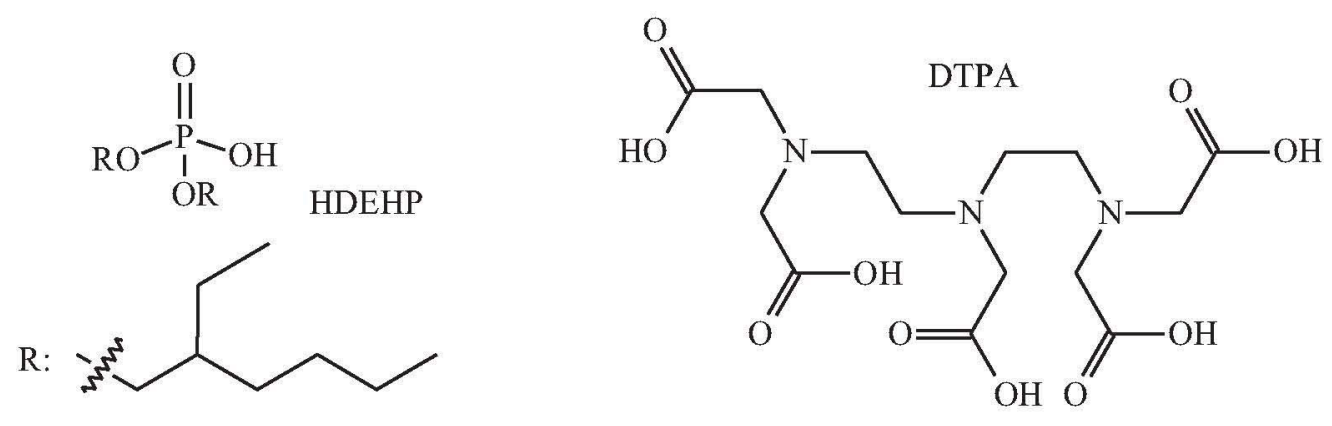

Figure 5. Structures of key components of TALSPEAK-type actinide/lanthanide separation, i.e. diethylenetriamine-N,N,N',N",N"-pentaacetic acid and di(2-ethylhexyl)phosphoric acid.

Figure 6 details the origins of the thermodynamic balance established between DTPA and HDEHP. When acting independently, the strength of complex formation for both reagents is governed predominantly by the increasing charge density of a trivalent lanthanide metal ion. The remarkable range of electrostatic interaction, spanning over 5 orders of magnitude across the lanthanide series, has been a signature of the phase transfer characteristics of HDEHP [19]. Examining the thermodynamic trend for the lanthanide de-complexation constants [20] conveys the influence of DTPA on the liquid-liquid distribution of lanthanides more clearly as such complexes must dissociate prior to phase transfer. The trend illustrates large differences in the bonding strength for the light members of the lanthanide series, while, largely due to the steric clashes, the complexation of heavier lanthanides by DTPA deviates from the predicted trend based on electrostatics. The thermodynamic trends presented this way illustrate the balancing relationship that guides the extraction of lanthanides in TALSPEAK-type separations. When a two-phase system lines up DTPA and HDEHP against each other the thermodynamic power struggle positions the reagents at the ends of the TALSPEAK lanthanide curve, where their influence is most prominent. The relative ease of dissociation of light lanthanide DTPA complexes enhances their partitioning into the organic phase, twisting up the linear $\mathrm{K}_{\mathrm{ex}}$ trend of HDEHP. This exact feature of DTPA-containing mixtures allows the separation of actinides from lanthanides and renders TALSPEAK successful. At the other end of this thermodynamic "see-saw" rests HDEHP, whose electrostatic interactions with the heavier lanthanides favour the extraction.

The clash between the holdback reagent and the phase transfer reagent presents an intriguing test ground for further examination of the described thermodynamic relationship. The TALSPEAK-type distribution system may be manipulated to study the shifting balance of lanthanide extraction curve as the experimental conditions interfere with an optimal performance of either reagent. Such strategic experimental complications illustrate a balancing act of thermodynamic forces that guide TALSPEAK separation. This study presents several liquid-liquid distribution exercises where the lanthanide complexation of DTPA was progressively switched-off as the $\mathrm{pH}$ of the aqueous phase was decreased. This interfering $\mathrm{pH}$ effect on the DTPA speciation was counterbalanced by employing polyaminophosphonates as more acidic holdback reagents to determine if the relationship persists. To disrupt the HDEHP-controlled end of the TALSPEAK lanthanide curve, the metal distribution was studied when the conventional aliphatic diluent was replaced by a depolymerizing aliphatic alcohol as an organic medium. 

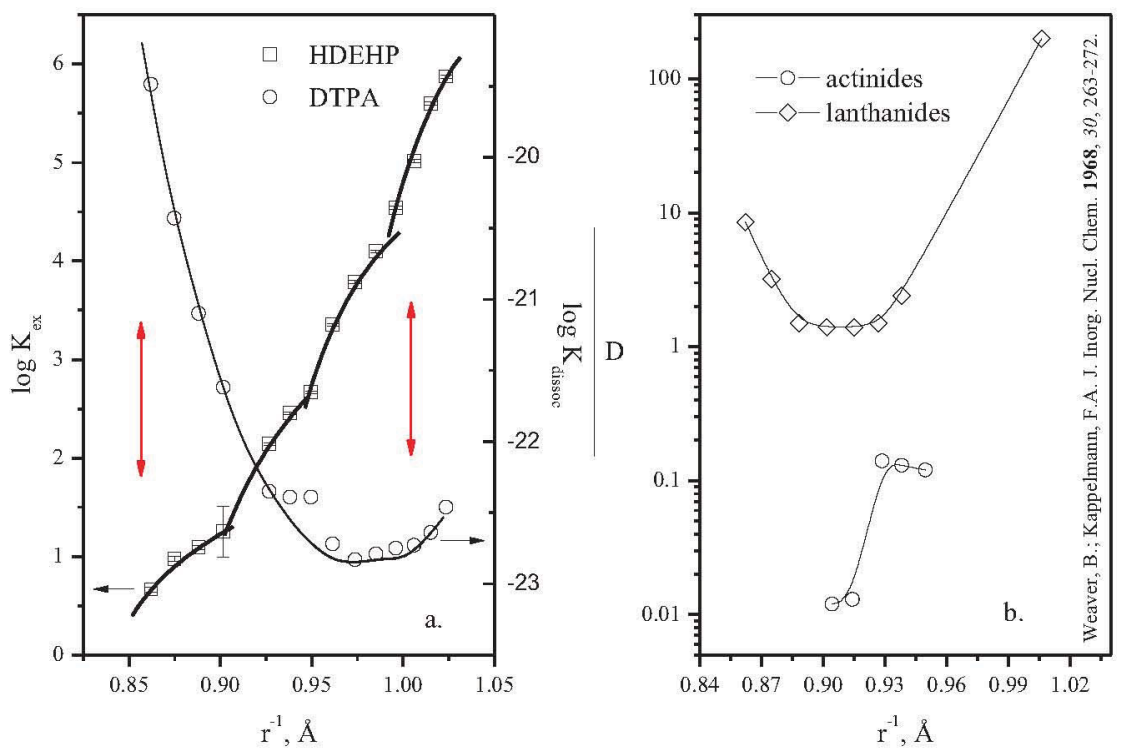

Figure 6 (a) Opposing thermodynamic forces in the TALSPEAK separation as quantified for lanthanide elements by extraction constants (HDEHP) and complex dissociation constants (DTPA), plotted as a function of reverse ionic radius. For extraction constants, org: HDEHP in n-dodecane, aq: $1.0 \mathrm{M}$ $\left(\mathrm{H}^{+}, \mathrm{Na}^{+}\right) \mathrm{ClO}_{4}$. Dissociation constants were taken from NIST database. (b) Final liquid-liquid distribution of actinides and lanthanides - a result of the thermodynamic struggle for balance between DTPA and HDEHP.

\subsubsection{Experimental}

The ${ }^{243} \mathrm{Am},{ }^{139} \mathrm{Ce}$ and ${ }^{154} \mathrm{Eu}$ radioisotopes were obtained from Idaho National Laboratory (INL) stocks. Pentasodium salt of DTPA (Alfa Aesar) and heptasodium salt of DTPP (Aldrich) were standardized using complexometric titration using a stock solution of europium nitrate (standardized using ICP-MS) and xylenol orange as an indicator. EDTP was purchased in acid form from Aldrich and sampled gravimetrically. Trisodium citrate, dimethyl glycine and ammonium sulfamate were purchased from Aldrich and used as received. Reagent grade $n$-dodecane (99\%), bis(2-ethylhexyl)phosphoric acid $(97 \%)$ and lactic acid $(85 \% \mathrm{w} / \mathrm{w})$ were purchased from Aldrich and used as received. Exxal 12 Dodecyl alcohol $(98 \%)$ was purchased from Exxon Mobile Chemical and used as received. Aqueous metal ion solutions were prepared using water from a Barnsted E-pure system and $70 \%(\mathrm{w} / \mathrm{w}) \mathrm{HNO}_{3}(\mathrm{Aldrich}$, Trace Metal Grade). All aqueous solutions contained 0.05 $\mathrm{M} \mathrm{Na}$ DTPA. Depending on the aqueous conditions a specific buffer was used, chosen for the proximity of a dissociation constant to $\mathrm{pH}$ under investigation. All solutions contained $1.0 \mathrm{M}$ buffer concentrations, and the $\mathrm{pH}$ was adjusted to 3.5 (lactate), 3 (citrate), 2 (dimethyl glycine) and 1 (sulfamate) using nitric acid (DTPA, DTPP) and sodium hydroxide (EDTP). The organic solutions of 0.1 M HDEHP in $n$-dodecane and 0.3 M HDEHP in Exxal 12 were prepared gravimetrically.

Metal extraction was quantified by the distribution ratio, D, calculated as the ratio of the equilibrium concentration of the radiotracer in the organic phase to that in the aqueous phase. The organic solutions of the extractant were pre-equilibrated with equal volumes of aqueous electrolyte solutions immediately prior to use. An aliquot of the pre-equilibrated organic phase was then contacted with an equal volume of the identical aqueous phase spiked with radiotracers. The two phases were vigorously shaken for 15 minutes at room temperature. The samples were centrifuged to facilitate phase separation and aliquot of each phase was taken for radiometric measurements using gamma spectrometry. 


\subsubsection{Results and Discussion}

Figure 7 illustrates the liquid-liquid distribution of $\mathrm{Am}(\mathrm{III}), \mathrm{Ce}(\mathrm{III})$ and $\mathrm{Eu}(\mathrm{III})$ for the investigated TALSPEAK-type systems, where the $\mathrm{pH}$ of aqueous phase was adjusted to 3,2 and 1, respectively. Acidity in the aqueous solutions was controlled by different buffering reagents, chosen for the proximity of their dissociation constants to the targeted $\mathrm{pH}$ condition. At $\mathrm{pH} 3$ the extraction behavior of the investigated metal ions parallels the expected outcome for TALSPEAK actinide/lanthanide separation.[16] Based on the distribution ratios for $\mathrm{Ce}(\mathrm{III})$ and $\mathrm{Eu}(\mathrm{III})$, an expected distribution curve for the entire lanthanide series may be re-constructed based on its usual proportions. Inspecting the trend, a separation factor between neodymium and americium was estimated at 21 , and the separation factor between cerium and americium was 68 . The accomplished separations, at the specified conditions, are indicative of well-balanced system of opposing forces in TALSPEAK. At $\mathrm{pH}$ of 3 an acid/base speciation of DTPA permits metal complexation, while HDEHP exerts a strong ion-exchanging influence. Consequently, all features of TALSPEAK separation are on display here, as the extraction of light lanthanides is enhanced and the americium is retained in the aqueous phase.
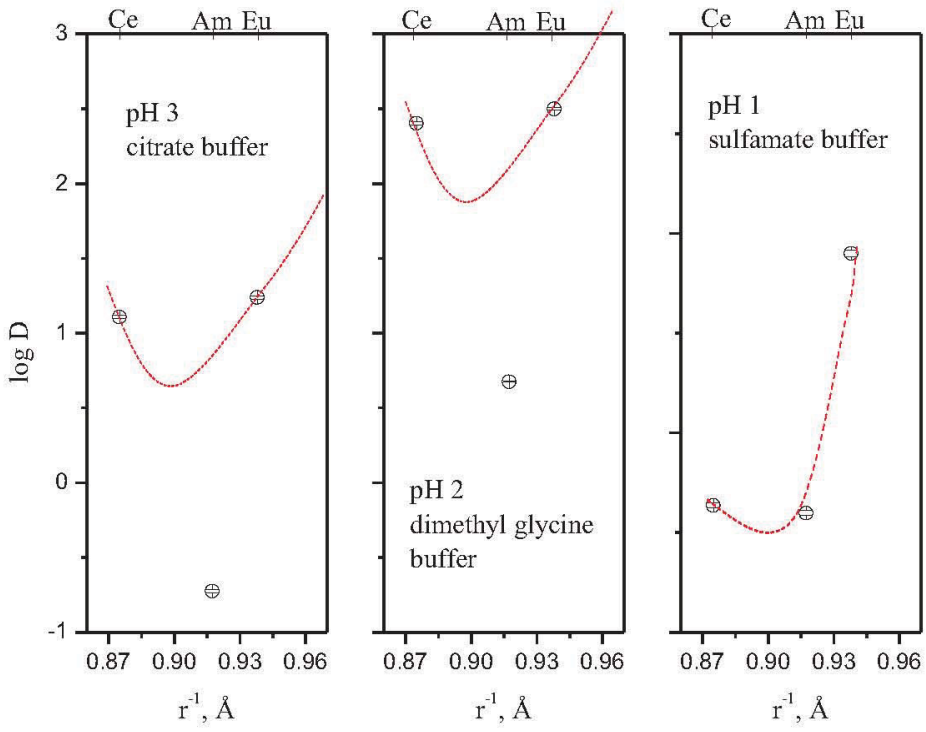

Figure 7. Distribution of Am(III), Ce(III) and Eu(III) for DTPA-containing TALSPEAK-type liquidliquid systems. The distribution curve for lanthanide ions (-) was reconstructed based on $\mathrm{D}_{\mathrm{Ce}}$ and $\mathrm{D}_{\mathrm{Eu}}$. Org: 0.1 M HDEHP in $n$-dodecane. Aq: $0.05 \mathrm{M}$ DTPA, 1.0 M appropriate buffer adjusted to $\mathrm{pH}$ under investigation.

When the $\mathrm{pH}$ of the aqueous phase was decreased to 2 , the separation failed. The thermodynamic balance between the DTPA and HDEHP is still maintained, judging from the consistent shape of the TALSPEAK lanthanide curve. However, aqueous complexation is now limited by the acid/base equilibria of DTPA (at pH of $250 \%$ exists as fully protonated acid) [20]. While the holdback influence of DTPA is compromised, it's preference for actinide coordination remains, yielding a separation factor of 19 between neodymium and americium. The dominance of HDEHP is exemplified by the significant enhancement of all metal distribution ratios, as the complexing power of DTPA is weakened by the increasing acidity.

The final and most adverse condition $(\mathrm{pH}=1)$ demonstrates a complete failure of the TALSPEAKtype separation, where beneficial complexing properties of DTPA are nearly completely switched off. 
The characteristic TALSPEAK lanthanide curve is distorted by the remaining coordinating force, tipping the balance of the thermodynamic "see-saw" in favour of HDEHP. As such the distribution ratios for the investigated metals shift in accordance with the expected linear trend for the extraction of the lanthanide series by HDEHP. The distribution of cerium decreases, relative to europium, and vice versa. As DTPA no longer interacts with americium its distribution is guided by electrostatic interaction with oxygendonating HDEHP. As the charge density of trivalent americium is similar to that of Pm(III) [21] no actinide/lanthanide separation is observed and the distribution ratio of americium is pulled up toward lanthanide trend. The overall decrease in the extraction efficiency for all metals may be attributed to a steep correlation $\left((\Delta \mathrm{D}) /\left(\Delta\left[\mathrm{H}^{+}\right]\right)=-3\right)$ between the HDEHP-facilitated ion-exchanging mechanism of phase transfer and the acidity of the aqueous phase.
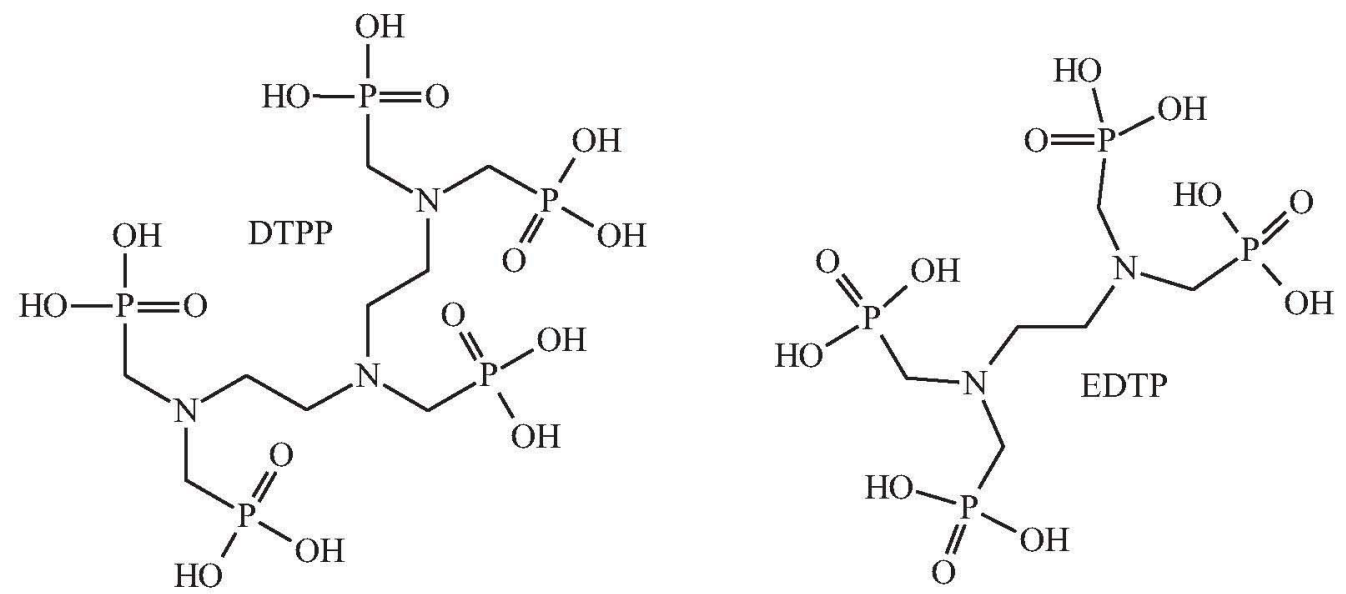

Figure 8. Structures of the aqueous phosphonic acid complexing agents investigated within TALSPEAKtype actinide/lanthanide separation framework, i.e. diethylenetriamine-N,N,N',N",N"'-pentaphosphonic acid, DTPP, and the ethylenediaminetetraphosphonic acid, EDTP.

To further investigate the effect of acidity, polyaminophosphonate holdback reagents were introduced into the system. Phosphonic acid functionality dissociates two protons and, due to the strong electroninducing influence of the initially dissociated proton, the second deprotonation step is much delayed, relative to the carboxylic acid. Accordingly, polyaminophosphonates are stronger acids, and, as such, their complexing behavior, i.e. holdback influence, should be extended into aqueous conditions of higher acidity. Figure 8 shows the structures of the diethylenetriamine-N,N,N',N",N"'-pentaphosphonic acid, DTPP, and the ethylenediaminetetraphosphonic acid, EDTP, employed in the study.

Figure 9 shows the distribution of $\mathrm{Am}$ (III), Ce(III) and Eu(III) between aqueous solutions of varying acidity, when DTPP was present as an aqueous complexant, and organic solution of HDEHP in $n$ dodecane. Inspecting the initial set of conditions ( $\mathrm{pH}$ 3), it is apparent that the phosphonate analog of DTPA fails to differentiate between actinides and lanthanides. As such, the presence of the phosphonic acid functionalities renders the complexing agent either too hard or too sterically hindered to allow for any weak covalent interactions with actinides. Also, the TALSPEAK lanthanide extraction curve is skewed in favour of HDEHP-controlled extraction at $\mathrm{pH}$ of 3 . Although the thermodynamic parameters for the complexation of lanthanides by aminopolyphosphonates are not available, it is reasonable to assume that at this acidity the holding-back capacity of DTPP is weaker, relative to DTPA, yielding to the phase transfer influence of HDEHP. Accordingly, a weaker influence of DTPP on the extraction of lanthanides results in the off-balanced thermodynamic relationship between the coordinating reagents. 

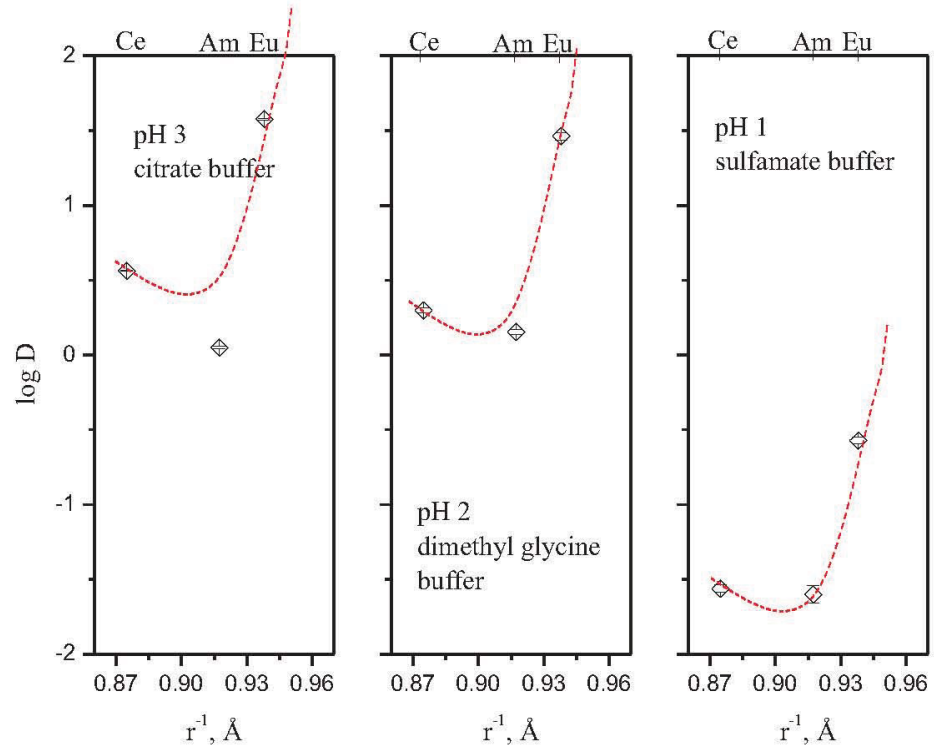

Figure 9. Distribution of $\mathrm{Am}$ (III), Ce(III) and $\mathrm{Eu}(\mathrm{III})$ for DTPP-containing TALSPEAK-type liquidliquid systems. The distribution curve for lanthanide ions $(-)$ was reconstructed based on $\mathrm{D}_{\mathrm{Ce}}$ and $\mathrm{D}_{\mathrm{Eu}}$. Org: 0.1 M HDEHP in $n$-dodecane. Aq: 0.05 M DTPA, 1.0 M appropriate buffer adjusted to $\mathrm{pH}$ under investigation.

As hypothesized, the holdback power of the aqueous complexing aminopolyphosphonate reagent is enhanced in the aqueous solutions of higher acidity. The metal distribution ratios are 1-2 orders of magnitude lower for the extraction of lanthanides from $\mathrm{pH} 2$ solutions, relative to those collected with DTPA in solution. However, as DTPP gains its aqueous complexation strength, the An/Ln differentiation is lacking, emphasizing the hardness of the phosphonate analog of DTPA. At a $\mathrm{pH}$ of 1 the combination of strong aqueous complexation and high hydrogen ion concentration significantly suppress the distribution ratios.

Very little difference in the liquid-liquid distribution of $\mathrm{Am}$ (III), $\mathrm{Ce}$ (III) and $\mathrm{Eu}$ (III) was observed when a smaller aminopolyphosphonate reagent - a phosphonic acid derivative of EDTA - was used as aqueous complexant. Figure 10 illustrates similarly-affected extraction trends, suggesting that steric crowding is a minor component of the observed solution behavior of these reagents. The comparable behavior of DTPP and EDTP indicate that both reagents are stronger acids, relative to DTPA, their holdback efficiency is higher at lower $\mathrm{pH}$, and their complexing powers are due to the purely electrostatic interactions. The aqueous complexation by EDTP is also reflected in the observed metal distribution ratios, which is a slightly weaker suppressant, relative to DTPP. 

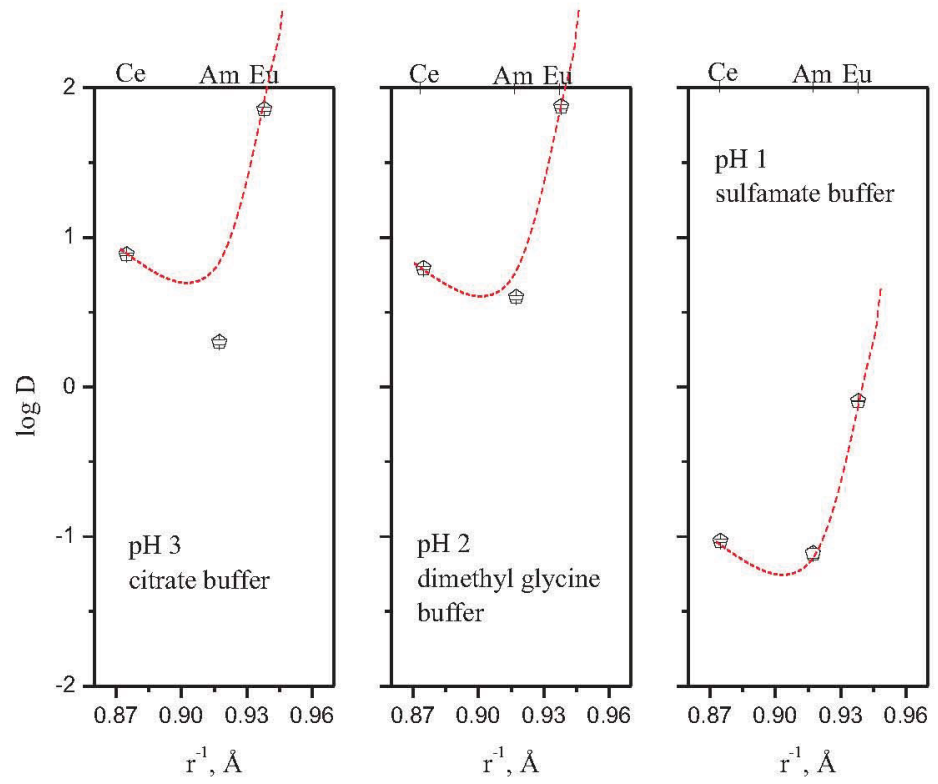

Figure 10. Distribution of Am(III), Ce(III) and Eu(III) for EDTP-containing TALSPEAK-type liquidliquid systems. The distribution curve for lanthanide ions (-) was reconstructed based on $\mathrm{D}_{\mathrm{Ce}}$ and $\mathrm{D}_{\mathrm{Eu}}$. Org: 0.1 M HDEHP in $n$-dodecane. Aq: 0.05 M DTPA, 1.0 M appropriate buffer adjusted to $\mathrm{pH}$ under investigation.

To shift the attention to the opposite end of the thermodynamic balancing act it is required to study the effects that complicate the extraction behavior of HDEHP. This organophosphate phase transfer reagent is a cation exchanger, which, in the non-aqueous environment, forms dimers and possibly higher aggregates at high HDEHP concentrations [22]. A well-characterized HDEHP-mediated mechanism of trivalent metal ion phase transfer involves a coordination of the metal by three extractant dimers and the release of three protons into the aqueous phase [23]. It has been shown previously that this mechanism of metal extraction is compromised in the presence of a depolymerizing alcoholic diluent, such as dodecanol [24]. As such, a substitution of $n$-dodecane for an aliphatic alcohol, when studying TALSPEAK-type separation systems, presents an opportunity to interfere with the normal phase transfer behavior of HDEHP.

Figure 11 compares the distribution of $\mathrm{Am}(\mathrm{III}), \mathrm{Ce}(\mathrm{III})$ and $\mathrm{Eu}(\mathrm{III})$ for TALSPEAK-type separation systems, where the aqueous phase is kept constant (0.05 M DTPA, $1.0 \mathrm{M} \mathrm{H}^{+} / \mathrm{Na}^{+}, \mathrm{Lac}^{-}, \mathrm{pH} 3.5,\left[\mathrm{Lac}^{-}\right]+$ $\left[\mathrm{Cl}^{-}\right]=1 \mathrm{M}$ ), and HDEHP is dissolved in aliphatic and alcoholic media. At $\mathrm{pH} 3.5$ the influence of DTPA's complexation chemistry grows as shown by the hypothesized TALSPEAK curve for lanthanide extraction. The trend in the distribution ratios for $\mathrm{Ce}(\mathrm{III})$ and $\mathrm{Eu}(\mathrm{III})$ is reversed, relative to the system studied at $\mathrm{pH} \mathrm{3}$, with cerium being extracted more strongely than europium. Separation factors are also reversed $\left(\mathrm{SF}_{\mathrm{Am}}{ }^{\mathrm{Ce}}=144, \mathrm{SF}_{\mathrm{Am}}{ }^{\mathrm{Eu}}=84\right.$ at $\mathrm{pH}=3.5, \mathrm{SF}_{\mathrm{Am}}{ }^{\mathrm{Ce}}=68, \mathrm{SF}_{\mathrm{Am}}{ }^{\mathrm{Eu}}=92$ at $\left.\mathrm{pH}=3.0\right)$ as DTPA strengthens its grip on light lanthanides. 


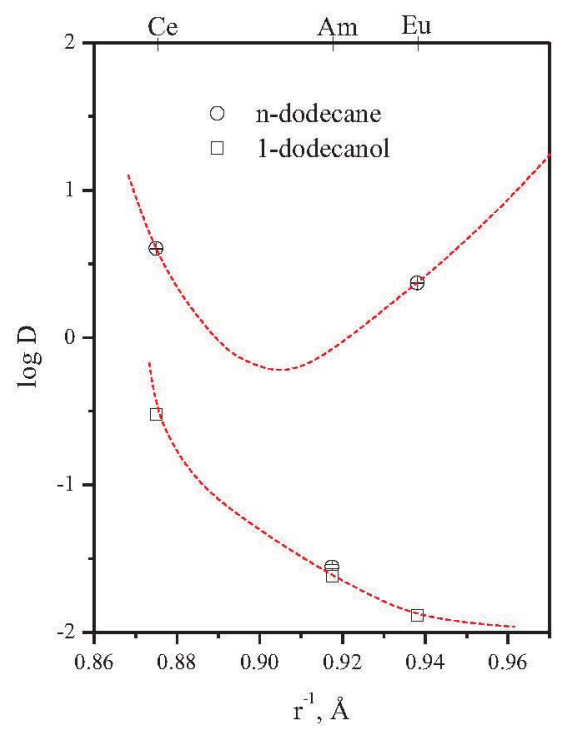

Figure 11. The comparison of distribution ratios for $\mathrm{Am}(\mathrm{III}), \mathrm{Ce}(\mathrm{III})$ and $\mathrm{Eu}(\mathrm{III})$ collected at normal aqueous TALSPEAK conditions (0.05 M DTPA, $\left.1.0 \mathrm{M} \mathrm{H}^{+} / \mathrm{Na}^{+}, \mathrm{Lac}^{-}, \mathrm{pH} 3.5\right)$, where an organic diluent varies between aliphatic hydrocarbon, $n$-dodecane, and aliphatic alcohol, 1-dodecanol.

A powerful phase transferring capacity of HDEHP is practically eliminated in a depolymerising alcoholic media. Dodecyl alcohol - a hydrogen bond donor - effectively coordinates with HDEHP, breaking up the extractant dimers. In this case the thermodynamic drive to metal ion transfer across the liquid-liquid boundary is significantly diminished as the formation of the polar solvation packet is more difficult. Figure 11 illustrates the significant loss of extraction efficiency by HDEHP. To collect measurable distribution ratios, the concentration of HDEHP was increased from $0.1 \mathrm{M}$ to $0.3 \mathrm{M}$ in the alcoholic media, further underlining the effect. Interestingly, the distribution of americium appears unaffected; reiterating that, at optimal acidity, liquid-liquid distribution of trivalent actinides in TALSPEAK systems is dominated by the aqueous complexation chemistry of aminopolycarboxylate reagent. In contrast, the extraction of lanthanides is significantly affected, with the efficiency of europium extraction suffering most.

With the elimination of strong the thermodynamic drive to phase transfer exerted by HDEHP, the thermodynamic balance is tipped in favour of the complexation by DTPA. At normal conditions the extraction of trivalent metals by HDEHP correlates well with the increasing effective charge density across the Ln series. As a result, the distribution of europium in the alcohol-modified TALSPEAK system drops significantly. Furthermore, owing to the relative ease of DTPA: $\mathrm{Ce}^{3+}$ complex dissociation the extraction of cerium is promoted, on a relative scale of the normal proportions of the TALSPEAK lanthanide curve. In consequence, the trend for the extraction of trivalent metal ions in a TALSPEAKtype system, where a depolymerising presence of alcohol turns off the usual mechanism of metal ion extraction by dimeric HDEHP, reverts to a thermodynamic trend of lanthanide/DTPA complex dissociation. It is worth noting that a stronger complexation of Am(III) by DTPA, relative to similar trivalent lanthanides, does not contribute to any meaningful enhancement in terms of $\mathrm{An} / \mathrm{Ln}$ separation unless HDEHP is correctly functioning in the 2-phase system. As such, it is the remarkable see-sawing balance between two powerful complexing agents that affords one of the most challenging of separations.

A superbly balanced thermodynamic struggle for metal ion coordination by the aqueous aminopolycarboxylate reagent DTPA and the non-aqueous organophosphorous phase transfer reagent, HDEHP, affords the separation of trivalent actinides from trivalent lanthanides. This thermodynamic 
relationship has been linked to an analogous "see-saw" behavior, where the balance is distorted when either of the key complexing players is subject to designed adverse conditions that interfere with their optimal operation. The thermodynamic balance is tipped in favour of HDEHP whenever increased acidity of the aqueous solution out-competes the metal ion complexation by aqueous complexing agent. When HDEHP is depolymerised due to the presence of aliphatic alcohol in the organic phase its phase transferring power is diminished. Such complication paves the way for DTPA to establish its dominance on the distribution of trivalent metal ions in the 2-phase system.

\subsection{First Calorimetric Determination of Heat of Extraction of ${ }^{248} \mathrm{Cm}$ in a Bi-phasic System}

\subsubsection{Approach}

As discussed previously, studying the thermodynamic properties of a solvent extraction system $\left(\Delta \mathrm{G}^{\circ}\right.$, $\Delta \mathrm{H}^{\circ}$ and $\Delta \mathrm{S}^{\circ}$ ) assists in providing an insight into the driving forces for the separation process, and possibly the end product. In some cases though, especially in the case of americium and curium separations, due to high radioactivity and limited supply of longer lived isotopes the physical and chemical measurements simply have not been performed. While tracer studies give a reasonable approximation of how the metal ion will behave in a separations system, they do not give absolute values. Those types of experiments require a direct measurement of the reaction under study.

Curium is one of the key actinides that is targeted for separation in the TALSPEAK process, but the chemical interactions of this element with the ligands that facilitate this process (lactic acid, DTPA, and di-2- ethyl hexyl phosphoric acid HDEHP) are, for reasons detailed above, simply not well defined. To that end, the study on the direct measurement of the heat of extraction of curium by HDEHP was highlighted as an area that could expand this field of research. By studying the thermochemistry of curium extraction from nitrate media by HDEHP using a calorimetric approach, we have begun to further expand our thermodynamic understanding of this liquid-liquid extraction system. Further it should be reiterated at this point that examples of these types of biphasic heats of extraction experiments are limited in the literature.

This is only the second such measurement with a transuranic element reported to date. To measure the biphasic heat of extraction of curium we have used the recently developed two-phase calorimetric methodology to study the thermochemical features of HDEHP-facilitated phase transfer of metal ions across a liquid-liquid boundary. This technique was successfully demonstrated with radioactive isotopes in FY-10 by measuring the heat of extraction of americium. In addition we have calculated $\Delta \mathrm{H}$ from the variation of the distribution ratio with temperature.[25] Isothermal titration calorimetry can accurately measure microjoule heat events inside of a $1 \mathrm{~mL}$ reaction vessel (Figure 12). The experiment followed the transfer of the $\mathrm{Cm}$ (III) ion from an aqueous nitrate solution into an organic phase containing bis(2ethylhexyl)phosphoric acid (HDEHP) - a hard electron donor dissolved in $n$-dodecane. The extraction of metal ions by HDEHP is a well characterized liquid-liquid partitioning system, utilizing a cation exchanging organophosphate phase transfer reagent. 


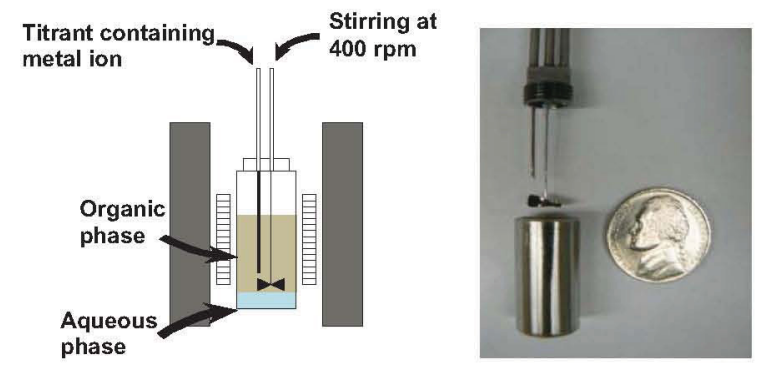

Figure 12. Schematic illustration of the calorimetric titration vessel, and calorimetric cup to scale.

When mildly acidic ( $\mathrm{pH} \sim 3$ ) aqueous solutions containing metal ions of the oxidation states II, III, and IV are contacted with the organic mixtures of HDEHP the hydration sphere of the metal is replaced by a number of coordinating oxygen donor groups of HDEHP. This reaction occurs at the aqueous/organic interface, where the metal is coordinated by three HDEHP dimers, the metal ion then crosses the phase boundary and enters the organic environment according to Equation 3 where HL is HDEHP and the horizontal bar represents species in the non-aqueous phase:[23]

$$
\mathrm{Cm}^{3+}+\overline{3(H A)_{2}} \Leftrightarrow \overline{\mathrm{Cm}\left(\mathrm{HA}_{2}\right)_{3}}+3 H^{+}
$$

Equation 3

The net enthalpy of reaction for the liquid-liquid partitioning of a metal ion across a phase boundary is the product of all of the enthalpy terms involved in the process including, but not limited to, metal ion dehydration, electrostatic bonding interactions and solvation of the newly formed extracted species in the organic phase. The overall change of energy in the system can be detected calorimetrically. The change in the enthalpy of transfer across the liquid-liquid boundary $-\Delta \mathrm{H}_{\mathrm{tr}}-$ describes the process quantitatively according to $\Delta \mathrm{H}_{\mathrm{tr}}=q / \Delta \mathrm{m}$, where $q$ is the calorimetrically detected heat associated with the reaction and $\Delta \mathrm{m}$ is the molar amount of solute that crosses the liquid-liquid interface.

Direct calorimetric measurement of $\Delta \mathrm{H}_{\mathrm{tr}}$ was afforded by careful design of experimental conditions to facilitate the liquid-liquid partitioning process. The calorimetric vessel contained both aqueous $(120 \mu \mathrm{L})$ and non-aqueous $(800 \mu \mathrm{L})$ solutions and the reference cell was also filled with $120 \mu \mathrm{L}$ nitrate solution and $800 \mu \mathrm{L}$ of organic phase. The aqueous phase consisted of $1.0 \mathrm{M}$ sodium nitrate adjusted to a $\mathrm{pH}$ of 3.0. The organic phase was a $0.2 \mathrm{M}$ solution of HDEHP in $n$-dodecane. The stirrer was rotating at $400 \mathrm{rpm}$ to achieve good mixing of the two phases. Sequential additions of $10 \mathrm{or} 20 \mathrm{uL}$ of the appropriate titrant solution introduced the metal ion under study into the calorimetric reaction vessel. Initially, the experiments were performed with samarium, a lanthanide element with an ionic radius similar to that of curium.[21] Two samarium conditions were tested prior to conducting the curium experiments. In the first experiment the samarium concentration in the titrant solution was $50 \mathrm{mM}$. As the curium concentration would be significantly lower than, this a further test experiment was conducted using $5 \mathrm{mM} \mathrm{Sm}$ in the titrant to observe if the heat responses would be adequate at these metal ion concentrations. The initial curium experiment, $20 \mathrm{uL}$ of $10.55 \mathrm{mM} \mathrm{Cm}$ in $1.0 \mathrm{M}$ sodium nitrate was injected into the calorimetric cup. The second experiment was performed using the same titrant but only injecting $10 \mathrm{uL}$ into the reaction vessel.

The van't Hoff analysis was conducted to measure the $\Delta \mathrm{H}$ of curium extraction from nitric acid media. Radio tracer concentrations of $\mathrm{Cm}^{3+}$ were added to an aqueous phase containing $0.2 \mathrm{M} \mathrm{HNO}_{3} / 0.8$ $\mathrm{M} \mathrm{NaNO}_{3}$ (creating an ionic strength of $1.0 \mathrm{M}$ ). The acidity of the aqueous phase was pH 1.58 to suppress the distribution ratios and prevent quantitative extraction of the $\mathrm{Cm}^{3+}$. The organic extractant di(2-ethylhexyl) phosphoric acid was dissolved in n-dodecane and the concentration was $0.2 \mathrm{M}$. All solutions were prepared by mass. 
The samples were all pre-equilibrated at room temperature and the extraction experiments were conducted at $10,20,25,30$, and $40^{\circ} \mathrm{C}$. The temperature dependence was achieved using a water jacketed beaker connected to a circulating water bath. The samples were allowed to come to thermal equilibration for $15 \mathrm{~min}$ prior to the vortex mixing. The samples were all shaken for a total of $10 \mathrm{~min}$ by alternating samples from the water jacketed beaker to the vortex mixer for 1 min intervals. After 10 min of shaking, the last sample was returned to the water jacketed beaker for 1 minute. This was followed by 1.5 minutes of centrifugation. The samples were again placed into the water jacketed beaker for $1 \mathrm{~min}$ before sampling of the phases to ensure the samples remained at the desired temperature throughout the duration of the experiments.

\subsubsection{Results}

Figure 13 shows the calorimetric heat response for the extraction of Sm(III) by HDEHP from sodium nitrate solution at $\mathrm{pH} 3.0([\mathrm{Sm}]=5 \mathrm{mM}$, injection volume $=20 \mu \mathrm{L})$. Each peak corresponds to the increasing heat transfer rate $(\mu \mathrm{W} / \mathrm{sec})$ as the calorimeter responds to the exothermic transfer of samarium ions across the liquid-liquid interface. Initial method development for calorimetric investigations of the heat of extraction of metal ions identified that other processes that accompany metal partitioning in a twophase system yield insignificant heat signatures. As such, the observed heat responses are solely responsible for the transfer of the metal ion across the phase boundary.[26]

The area under the peak can be integrated to give a net change of energy in the system $(q)$, which is directly related to the concentration of metal ion titrated into the calorimetric reaction vessel via Equation 4.

$$
\Delta H=\frac{q}{\Delta m}
$$

Equation 4

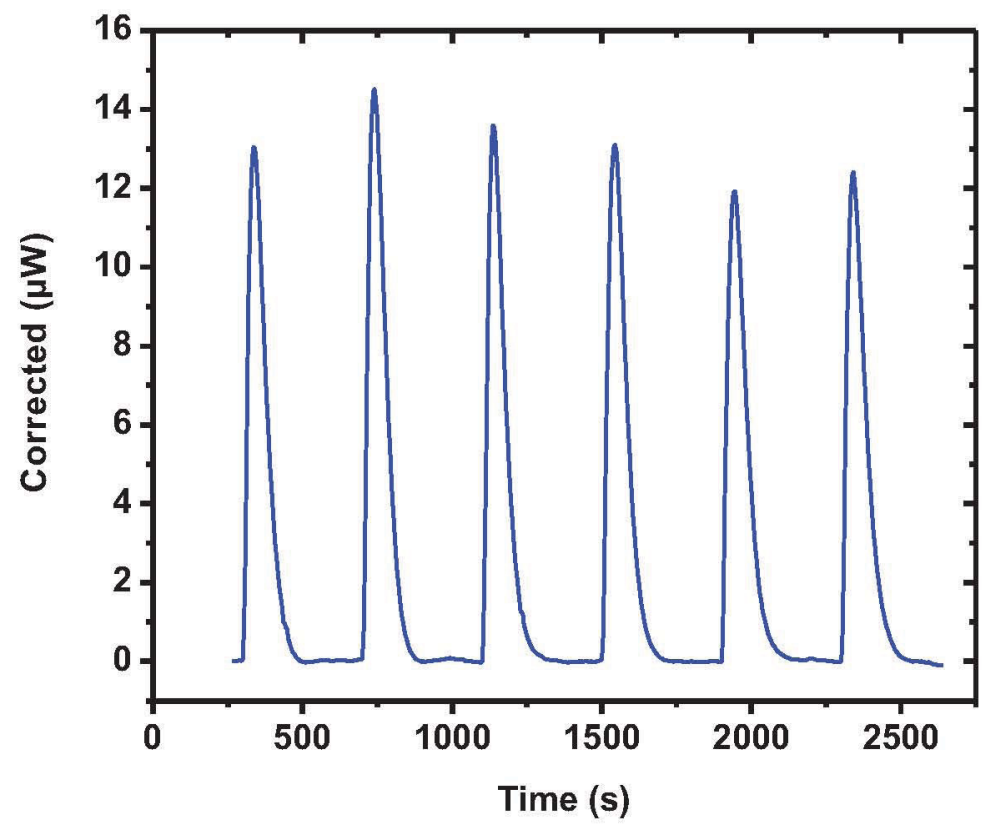

Figure 13. Calorimetric power compensation trace collected for the liquid-liquid distribution of Sm(III). Aqueous phase: $\mathrm{pH} 3.0,\left[\mathrm{NaNO}_{3}\right]=1.0 \mathrm{M},[\mathrm{Sm}(\mathrm{III})]=5 \mathrm{mM}$. Organic phase: $0.2 \mathrm{M}$ HDEHP in $n$ dodecane, titrant injection size $=20 \mu \mathrm{L}$. 
For the two samarium experimental conditions studied (titrant metal concentration of $50 \mathrm{mM}$ and 5 $\mathrm{mM}$ ) the average heat of extraction obtained was $-10.7 \pm 1.0 \mathrm{~kJ} \mathrm{~mol}^{-1}$. This value is in reasonable agreement with the previously obtained value $-10.9 \mathrm{~kJ} \mathrm{~mol}^{-1}$, obtained by Zalupski using the same methodology at Washington State University (WSU).[26] With the setup and methodology at INL reproducing viable results, the curium experiments were conducted. Figure 14 shows the power compensation trace for the extraction of $10.55 \mathrm{mM}$ curium into $0.2 \mathrm{M}$ HDEHP in dodecane. To obtain reasonable heat compensation traces in the initial investigation $20 \mu \mathrm{L}$ injections of titrant were made as with the samarium experiment. On inspection of Figure 14, it can be seen that the heat responses were significantly higher than those obtained in the samarium experiments. As such, the subsequent experiment performed used a $10 \mu \mathrm{L}$ sample injection size (Figure 15). One qualitative point of note is that in the curium experiments there is some tailing on the peaks observed. This feature notably extends the time to complete the experimental heat of extraction determination. It is worthy of mention here that this was not observed in the americium experiments that were conducted in FY-10 and possibly could be due to kinetic differences in the extraction of curium over the other elements that have been studied using this technique. Further solvent extraction experiments would be required to verify that this is the case. The results for all of the heats of extraction experiments are shown in Table 1. Figure 16 shows the experimentally determined heats of extraction for curium by isothermal titration calorimetry compared to those determined for the lanthanides and americium using the same technique.

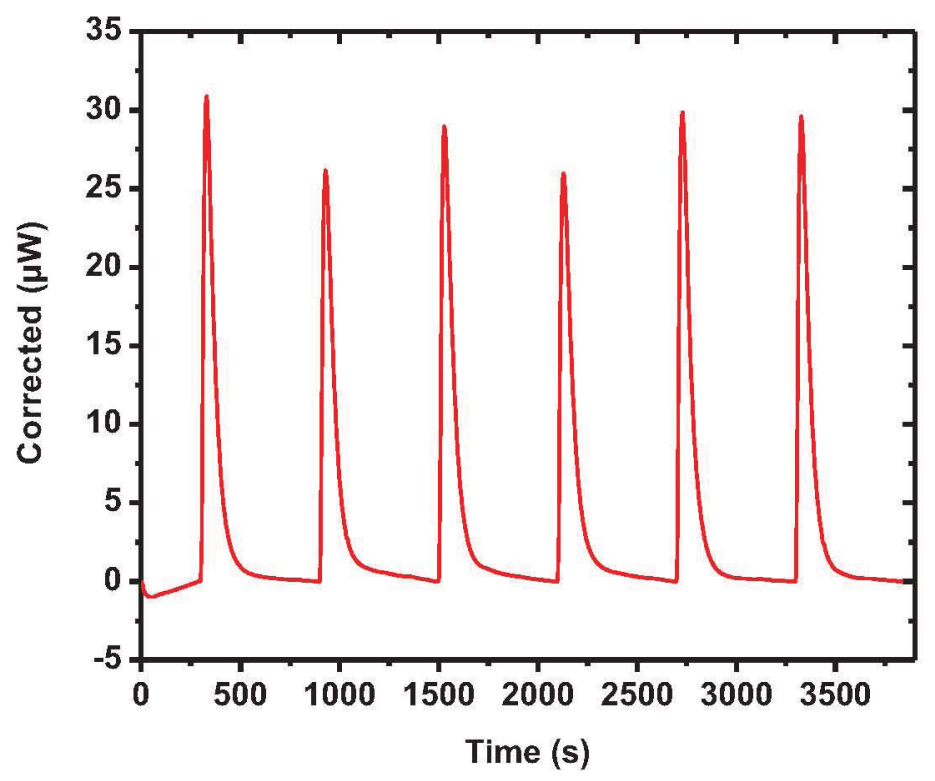

Figure 14. Calorimetric power compensation trace collected for the liquid-liquid distribution of Cm(III). Aqueous phase: $\mathrm{pH} 3.0,\left[\mathrm{NaNO}_{3}\right]=1.0 \mathrm{M},[\mathrm{Cm}(\mathrm{III})]=10.55 \mathrm{mM}$. Organic phase: $0.2 \mathrm{M}$ HDEHP in $n$ dodecane, titrant injection size $=20 \mu \mathrm{L}$. 


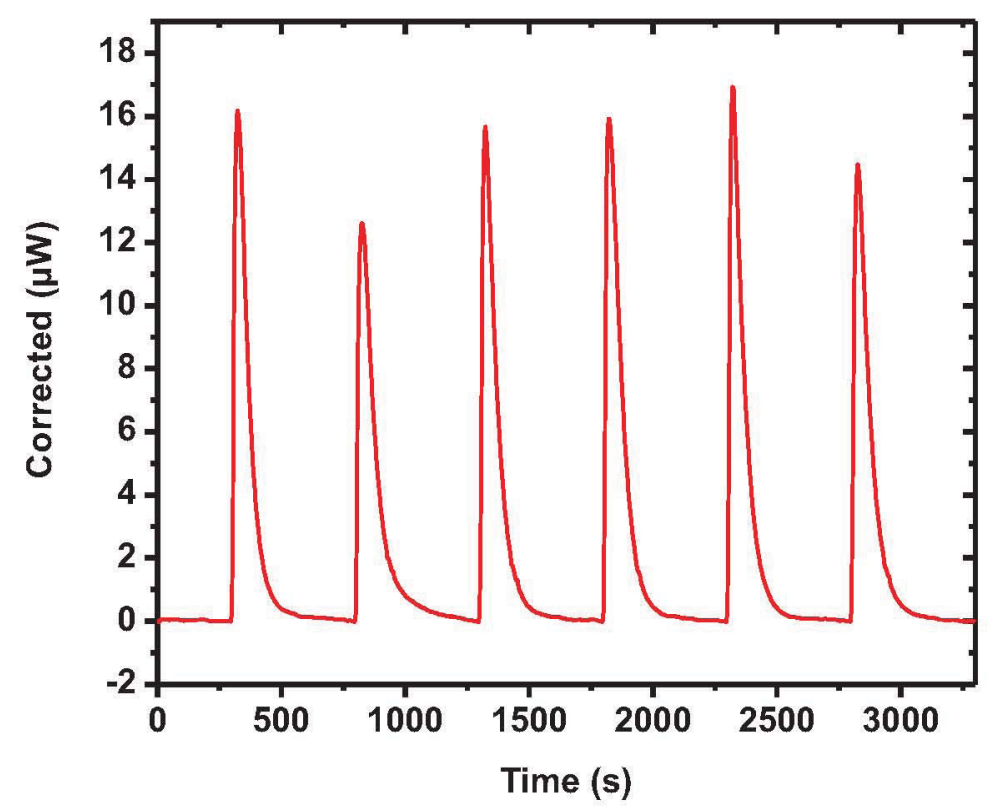

Figure 15. Calorimetric power compensation trace collected for the liquid-liquid distribution of $\mathrm{Cm}$ (III). Aqueous phase: $\mathrm{pH} 3.0,\left[\mathrm{NaNO}_{3}\right]=1.0 \mathrm{M},[\mathrm{Cm}(\mathrm{III})]=10.55 \mathrm{mM}$. Organic phase: $0.2 \mathrm{M}$ HDEHP in $n$ dodecane, titrant injection size $=10 \mu \mathrm{L}$.

Table 1. Heats of extraction and errors determined for samarium and curium in the biphasic calorimetry experiments.

\begin{tabular}{|c|c|c|c|c|}
\hline Experiment & $\begin{array}{c}\text { Average peak } \\
\text { area } / \mu \mathrm{J}\end{array}$ & $\begin{array}{l}\text { Standard } \\
\text { deviation }\end{array}$ & $\%$ RSD & $\begin{array}{c}\Delta \mathrm{H} \text { extraction } / \\
\mathrm{kJ} \mathrm{mol}^{-1}\end{array}$ \\
\hline $\begin{array}{l}{[\mathrm{Sm}]=50 \mathrm{mM}} \\
10 \mu \mathrm{L} \text { injection }\end{array}$ & -5838.08 & 228.96 & -3.9 & $-11.7 \pm 0.9$ \\
\hline $\begin{array}{l}{[\mathrm{Sm}]=5 \mathrm{mM},} \\
20 \mu \mathrm{L} \text { injection }\end{array}$ & -957.40 & 36.37 & -3.8 & $-9.6 \pm 0.7$ \\
\hline $\begin{array}{l}{[\mathrm{Cm}]=6.6 \mathrm{mM},} \\
20 \mu \mathrm{L} \text { injection }\end{array}$ & -2107.23 & 70.12 & -3.3 & $-10.0 \pm 0.7$ \\
\hline $\begin{array}{l}{[\mathrm{Cm}]=6.6 \mathrm{mM},} \\
10 \mu \mathrm{L} \text { injection }\end{array}$ & -1110.09 & 50.52 & -4.6 & $-10.5 \pm 1.0$ \\
\hline
\end{tabular}




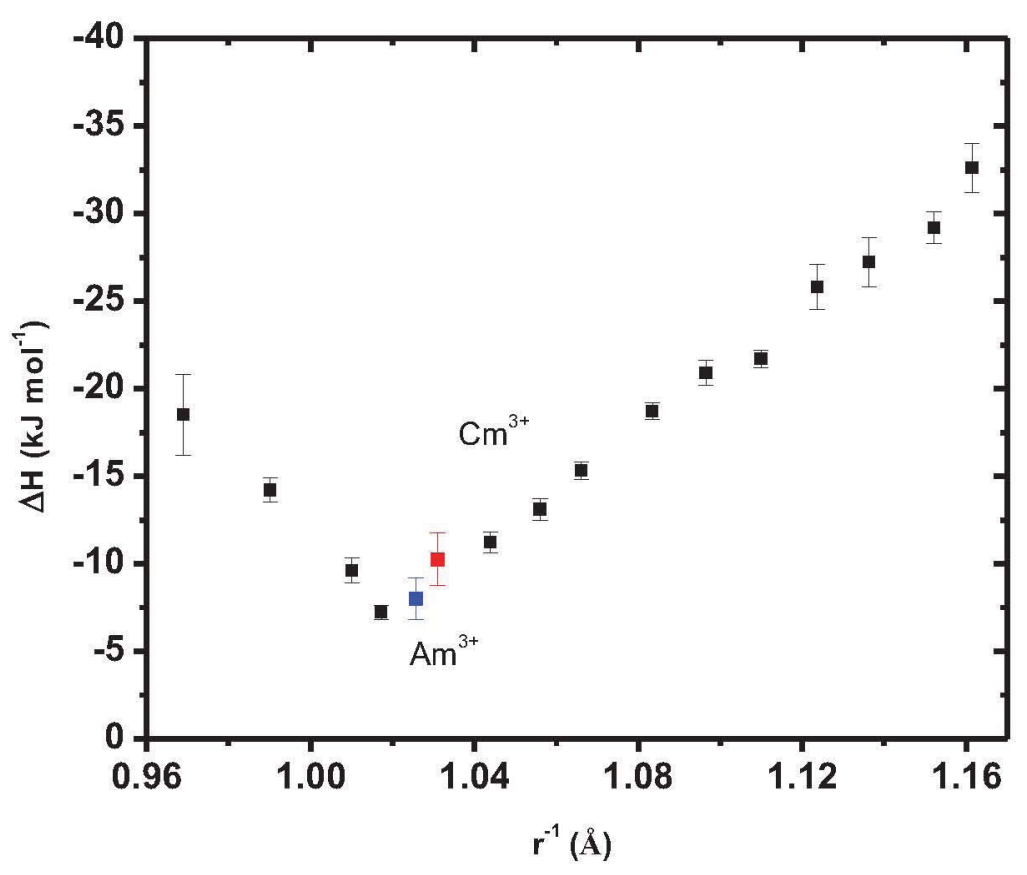

Figure 16. The comparison of the heat of extraction for the HDEHP-facilitated liquid-liquid partitioning of $\mathrm{Cm}$ and $\mathrm{Am}$ with those reported for the lanthanide series as a function of ionic radii for 6 coordinate metal ion.

The heats of extraction of curium measured using isothermal titration calorimetry using the two different injection volumes were internally consistent $\left(-10.00\right.$ and $\left.-10.50 \mathrm{~kJ} \mathrm{~mol}^{-1}\right)$ highlighting the consistency of this technique. To complement this work, solvent extraction experiments were performed as a function of temperature. The results of the variation of distribution ratio as a function of temperature are shown in Figure 17. The plot of $K_{\text {ex }}$ vs $1 / T$ results in a straight line with the slope of $-\Delta H / R$. The $\Delta H$ of extraction calculated from this set of experiments was determined to be $-7.2 \pm 0.38 \mathrm{~kJ} \mathrm{~mol}^{-1}$. The Gibbs free energy of curium transfer in this solvent extraction system was calculated using Equation 5:

$$
\Delta G_{t r}=-R T K_{e q}
$$

Equation 5

Using $\mathrm{K}_{\text {eq }}$ at $25^{\circ} \mathrm{C}$ the $\Delta \mathrm{G}_{\mathrm{tr}}$ was found to be $-1.58 \mathrm{~kJ} \mathrm{~mol}^{-1}$, the entropy term was calculated from the calorimetrically obtained $\Delta \mathrm{H}_{\mathrm{tr}}$ value and the above calculated $\Delta \mathrm{G}_{\mathrm{tr}}$ value using Equation 6

$$
\Delta S_{t r}=\left(\frac{\Delta H_{t r}-\Delta G_{t r}}{T}\right)
$$

Equation 6

The entropy at $25^{\circ} \mathrm{C}$ for this system was found to be $-23.23 \mathrm{~J} \mathrm{~mol}^{-1} \mathrm{~K}^{-1}$

Although the two methodologies for measuring the heat of extraction of curium resulted in statistically different values, they are in reasonable agreement with each other. The heat of extraction of americium that was determined in FY-10 using this technique, yielded a value of $-8.0 \pm 1.2 \mathrm{~kJ} / \mathrm{mol}( \pm 2 \sigma)$. When comparing the calorimetrically determined values alone we find good agreement in the trend that is observed with the extraction of the lanthanide series of elements. In the lanthanides series, it is observed that there is an increase of $\sim 2 \mathrm{~kJ} \mathrm{~mol}^{-1}$ for the heat of extraction between adjacent lanthanides.[26] 


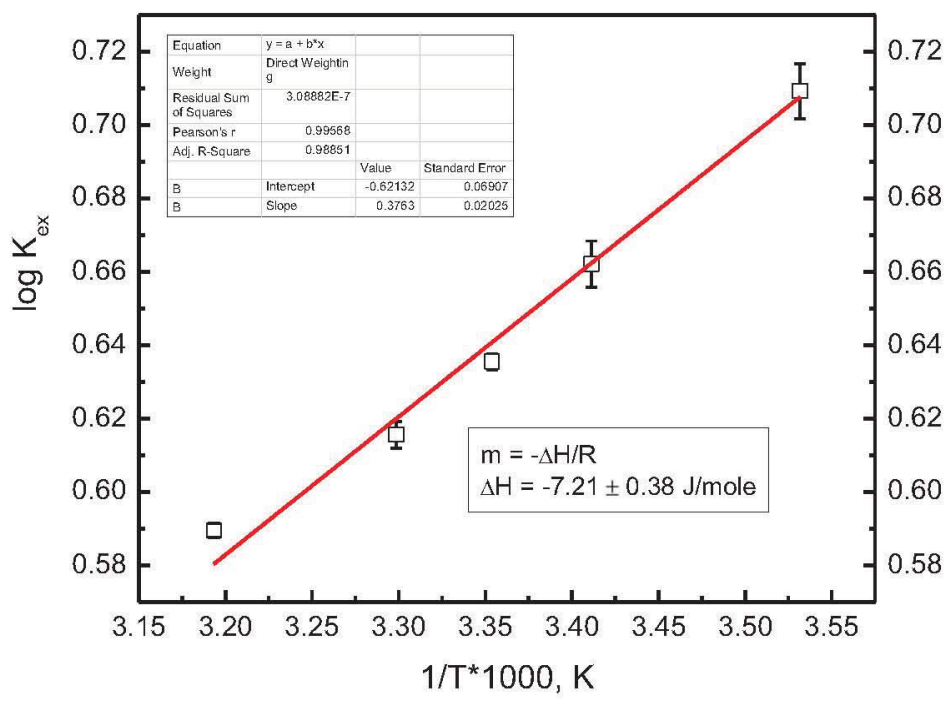

Figure 17. Liquid-liquid distribution of tracer $\mathrm{Cm}(\mathrm{III})$ as a function of temperature. Aqueous phase: $\mathrm{pH}$ $1.58,\left[\mathrm{NaNO}_{3}\right]=1.0 \mathrm{M},[\mathrm{Cm}(\mathrm{III})]=$ tracerM . Organic phase: $0.2 \mathrm{M}$ HDEHP in $n$-dodecane.

\subsection{Kinetics}

The success of a solvent extraction system for the separation of any metal ion from aqueous solution often relies on the kinetics of the chemical processes involved. If the chemical reactions that facilitate separations take too long the process has little scale-up potential. Further, studying kinetics can yield mechanistic information about the processes involved in the separation that thermodynamics simply cannot. In TALSPEAK complexation, reactions in the aqueous phase and phase transfer kinetics both have to be considered. As such, building on the interesting results observed in this program in FY-10 the work performed this year has continued to investigate improved ways to study aqueous phase complexation kinetics and further investigated the phase transfer kinetics of the lanthanide ions.

\subsubsection{Aqueous Phase Complexation Kinetics}

Aqueous phase complexation and decomplexation reactions of the actinides and lanthanides have been suggested as the rate determining step for the TALSPEAK separation.[10-11] However, studying these reactions using the complexometric dye arsenazo(III) has proved problematic, with multiple reactions being identified.[9] In FY 11 two new dyes, Eriochrome Black T (EBT) and Xylenol orange (XO) have been investigated for applicability in monitoring the aqueous phase binding kinetics of DTPA to the lanthanide elements. Initially UV-visible spectra of each dye and each Eu-dye complex were recorded to find sufficiently different absorbances to allow kinetic measurements to be performed. These spectra can be seen in Figure 18 a and b. Subsequent experiments were performed using different concentrations of each dye to determine the extinction coefficients for both EBT and XO ( Figure $19 \mathrm{c}$ and $\mathrm{d}$ ). 

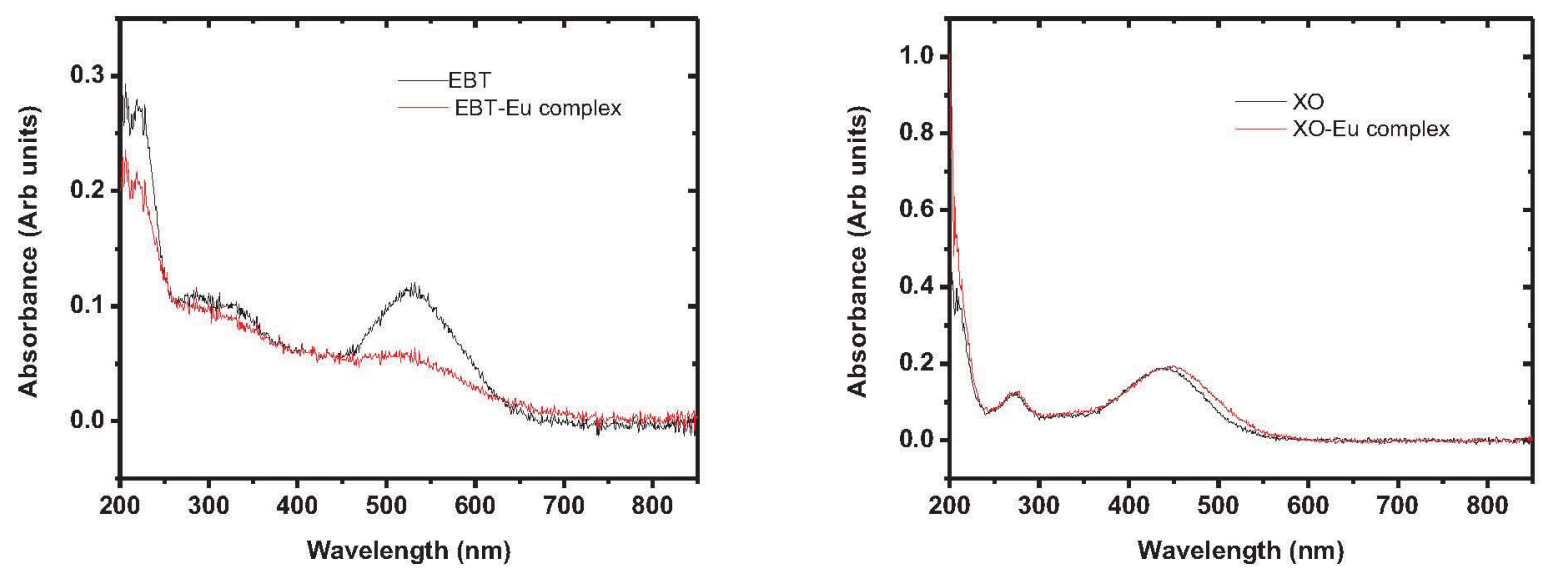

Figure 19 UV-visible spectra of a) bound and unbound EBT $[\mathrm{Eu}]=1.0 \times 10^{-5} \mathrm{M},[\mathrm{EBT}]=1 \times 10^{-5} \mathrm{M}$, and b) bound and unbound $\mathrm{XO}[\mathrm{Eu}]=1 \times 10^{-5} \mathrm{M},[\mathrm{XO}]=1 \times 10^{-5} \mathrm{M}$,
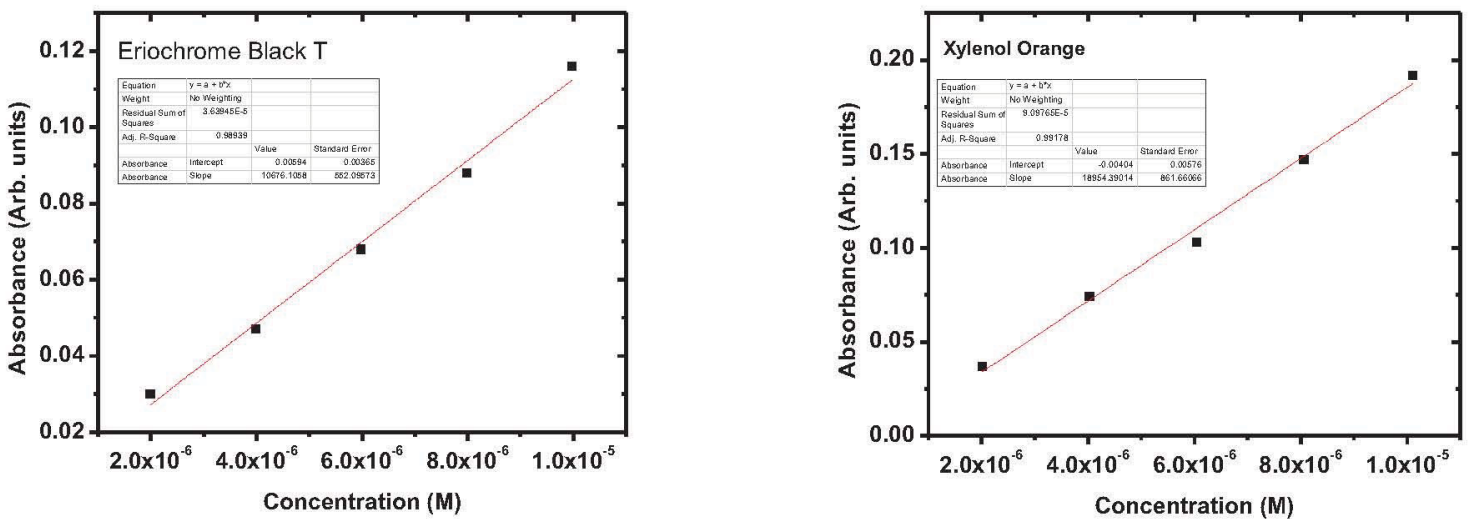

Figure 18 Beers law plots to determine the extinction coefficient for a)EBT and b) Xylenol orange

It was determined that due to the significant differences in spectra between the bound and unbound form of EBT, this dye would be carried forward into the stopped flow spectrophotometry experiments.

Examples of the stopped flow kinetics traces obtained on a HI-TECH SF-61 DX2 stopped flow spectrophotometer using EBT to monitor the kinetics of binding of DTPA to europium are shown in Figures 20 and 21. Similar to the results found with Arsenazo(III) previously, the kinetic traces could not be adequately fit using the first order rate equation, Equation 7 but instead required a double exponential growth, Equation 8, to better describe the kinetics observed.

$$
\begin{gathered}
y=A_{1} \times e^{\left(k_{1} x\right)}+B \\
y=A_{1} \times e^{\left(k_{1} x\right)}+A_{2} \times e^{\left(k_{2} x\right)}+B
\end{gathered}
$$$$
\text { Equation } 7
$$ 


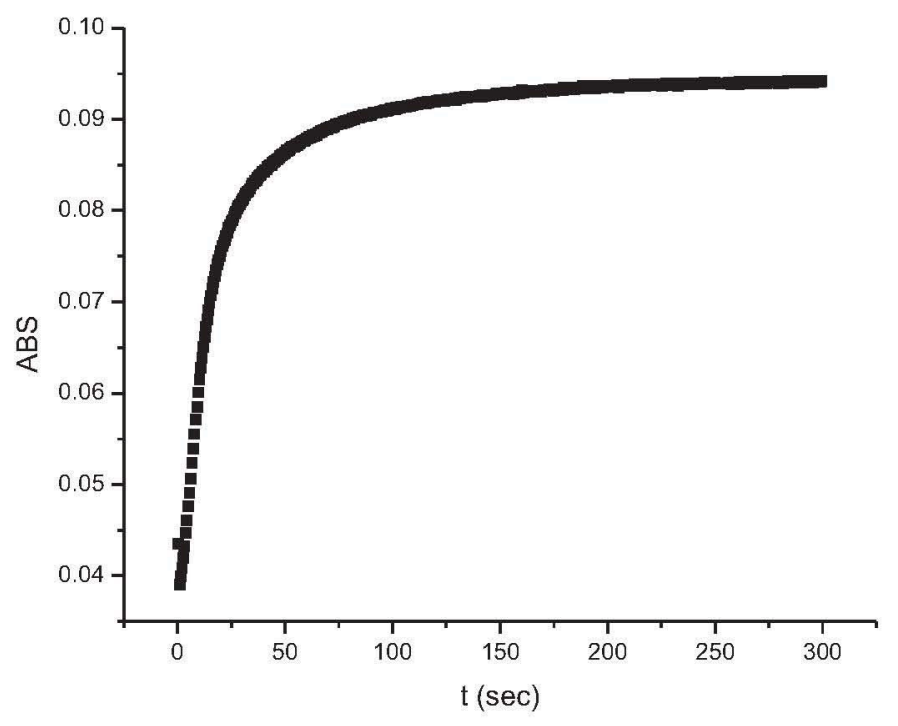

Figure 20. Sample kinetics trace from the stopped flow spectrophotometer measuring the rate of reaction of Eu-EBT complex with DTPA. $[\mathrm{Eu}]=5 \times 10^{-6} \mathrm{M},[\mathrm{EBT}]=1 \times 10^{-5} \mathrm{M},[\mathrm{DTPA}]=1.25 \times 10^{-3} \mathrm{M}, \mathrm{pH} 3.6$.

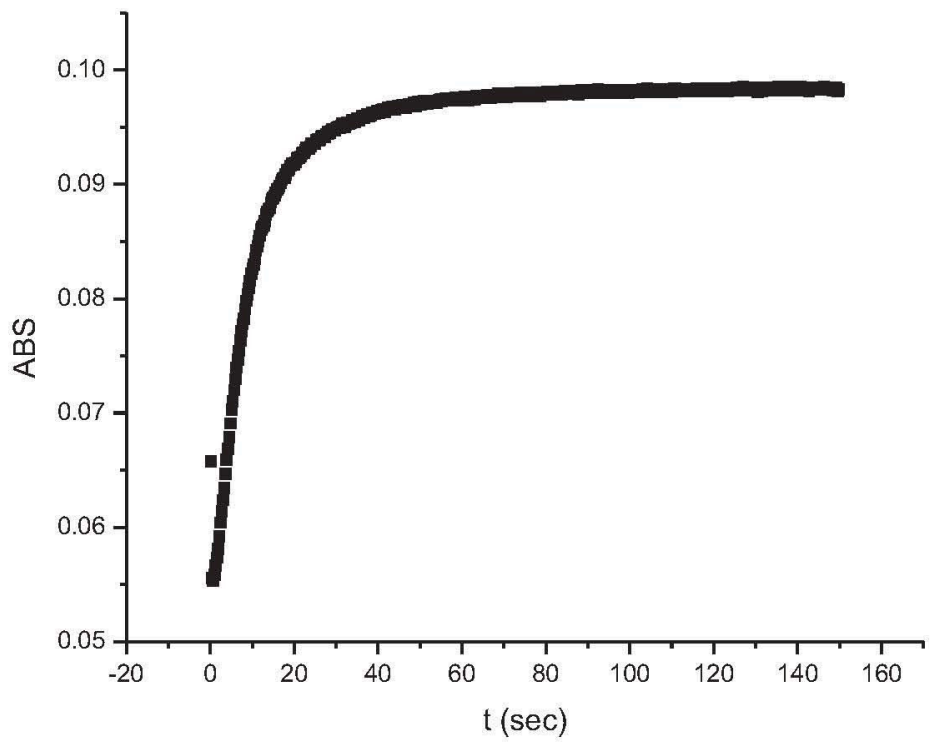

Figure 21. Sample kinetics trace from the stopped flow spectrophotometer measuring the rate of reaction of Eu-EBT complex with DTPA. $[\mathrm{Eu}]=5 \times 10^{-6} \mathrm{M},[\mathrm{EBT}]=1 \times 10^{-5} \mathrm{M},[\mathrm{DTPA}]=5.00 \times 10^{-3} \mathrm{M}, \mathrm{pH} 3.6$. DTPA 500, 5uM Eu, 10uM EBT (dye) 
For the above conditions described by Figures 20 and 21, the growth fit data are tabulated in the table below.

Table 2. Average kinetic parameters determined for the reaction of DTPA with Eu

\begin{tabular}{cccccc}
\hline$[\mathrm{DTPA}](\mu \mathrm{M})$ & $\mathrm{k}_{1}$ & $\mathrm{k}_{2}$ & $\mathrm{~A}_{1}$ & $\mathrm{~A}_{2}$ & $\mathrm{~B}$ \\
\hline 125 & $0.080 \pm 0.001$ & $0.015 \pm 0.001$ & $0.046 \pm 0.001$ & $0.014 \pm 0.001$ & $0.034 \pm 0.001$ \\
250 & $0.284 \pm 0.006$ & $0.035 \pm 0.001$ & $0.038 \pm 0.001$ & $0.019 \pm 0.001$ & $0.053 \pm 0.001$ \\
500 & $0.113 \pm 0.003$ & $0.018 \pm 0.006$ & $0.043 \pm 0.001$ & $0.004 \pm 0.001$ & $0.052 \pm 0.001$ \\
700 & $0.232 \pm 0.003$ & $0.027 \pm 0.001$ & $0.045 \pm 0.001$ & $0.008 \pm 0.001$ & $0.069 \pm 0.001$ \\
\hline
\end{tabular}

Furthermore, it has been identified during the course of these investigations that the propionic acid buffer used also interfered with the Eu-EBT complexation. Considerably slower kinetics were obtained in the absence of this buffer, but maintaining solution $\mathrm{pH}$ was very difficult. As it is of significant importance to more accurately describe the kinetics of complexation between DTPA and the trivalent lanthanides and actinides in solution, further experimentation that simplify the system is required. This further experimentation will include, but is not limited to, using different $\mathrm{pH}$ buffers such as hexamethylenetetramine that will not directly interact with the metals or DTPA and different dyes (methyl red, ABTS) that complex less strongly to the metal ions.

\subsubsection{TALSPEAK Solvent Extraction Kinetics}

Our FY10 studies into the kinetics of extraction of europium and cerium in a "standardized" TALSPEAK system lead to some interesting results that identified the kinetics of extraction of tracer cerium was not only dependent on the concentration of DTPA in the aqueous phase, but also the presence of macro europium had a significant effect on its extraction behavior. This phenomenon has not previously been observed, largely due to the fact that most literature studies have been performed using tracer metal ion instead of macro metal ions in solution. As such the investigations pursued in FY11 have moved to develop these ideas further by probing the effects of initial metal ion concentration in the aqueous phase. In summary, the experiments to study the kinetics of phase transfer of macro $\mathrm{Ce}$, tracer Eu and macro cerium/ macro europium were set up as follows:

Aqueous phase

- $1 \mathrm{M}$ total lactate $\mathrm{pH}$ adjusted to 3.8

- Varying DTPA concentration 10,20,4050 mM

- $10 \mathrm{mM} \mathrm{Ce}$, tracer Eu

Organic phase

- $0.17 \mathrm{M}$ HDEHP in n-dodecane

Mixing

- All organic phases were pre-equilibrated with appropriate aqueous phases

- A rotating bar was used to mix the two phases for contact times of $15,30,60,120$ and 180 mins. 
Distribution ratios of europium and cerium as a function of mixing time are presented in Figures 22 and 23 where the initial aqueous phase concentration of cerium was $10 \mathrm{mM}$.

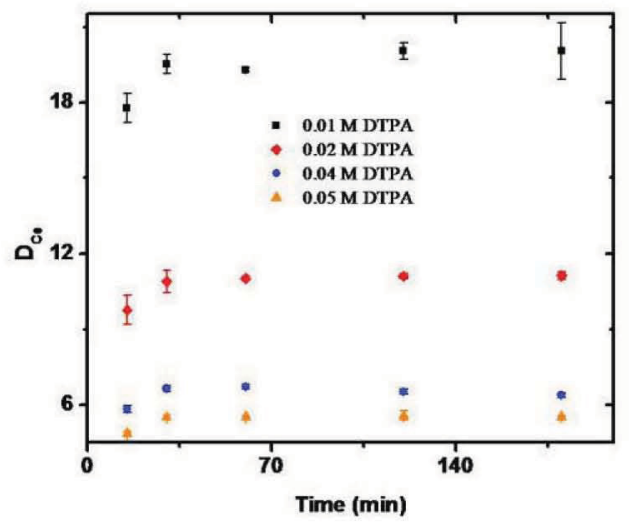

Figure 22. Influence of $[\mathrm{DTPA}]_{(\mathrm{aq})}$ on rate of extraction of cerium by $0.17 \mathrm{M}$ HDEHP in dodecane using a rotary mixer to agitate the two phases. Aqueous phase composition $1 \mathrm{M}$ lactic acid at $\mathrm{pH} 3.8,[\mathrm{DTPA}] 10-50 \mathrm{mM},[\mathrm{Ce}] 10 \mathrm{mM}$.

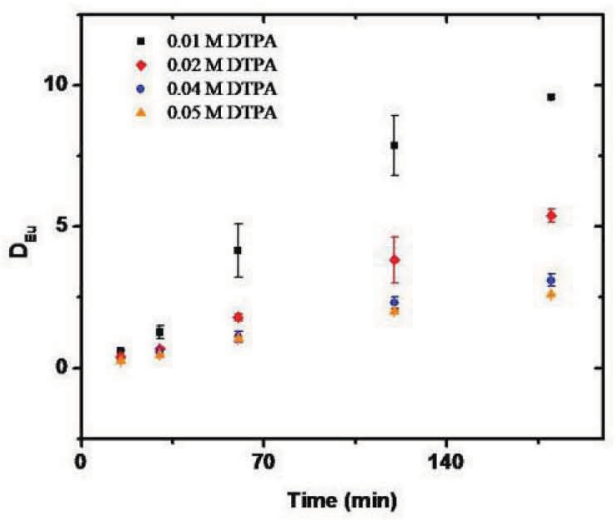

Figure 23. Influence of $[\mathrm{DTPA}]_{(\mathrm{aq})}$ on rate of extraction of tracer $\mathrm{Eu}$ by $0.17 \mathrm{M}$ HDEHP in dodecane using a rotary mixer to agitate the two phases. Aqueous phase composition 1.0 M lactic acid at $\mathrm{pH} 3.8$, [DTPA] $10-50 \mathrm{mM}$, [Ce] $10 \mathrm{mM}$.

The data presented in Figure 22 show when there is millimolar cerium present initially in the aqueous phase a more "classical" extraction profile is observed with the cerium increasing to an equilibrium point after about 30 mins contact time. This is in contrast to the behavior reported in FY10 [9] where the cerium is rapidly extracted and the distribution value decreases as more europium is extracted into the organic phase. Figure 23 also show a slightly different result for europium behavior in this system, with it taking almost an hour longer for the distribution to come to equilibrium. The cerium behavior observed here more closely resembles what would be expected from the prior literature i.e. rapidly reaching equilibrium.[7] However, the suppression of the europium extraction kinetics only serves to reiterate that the kinetics of extraction of metal ions in TALSPEAK type media is greatly reliant on the initial aqueous phase composition. It should be noted that the distribution values shown here are similar to those reported in FY 10. This would indicate that under the conditions studied here there is no significant loading of the organic phase.

The results of the experiments in which the aqueous phase contained $10 \mathrm{mM} \mathrm{Ce}$ and $10 \mathrm{mM} \mathrm{Eu}$ are shown in Figures 24 and 25. The first major point of note in this system is that both metal ions come to equilibrium between 1-2 hours of mixing on the rotating bar, this is significantly faster than either of the previous scenarios studied where only one metal ion is present at millimolar concentrations. However, on increasing the europium concentration in the aqueous phase there is a return to the trend observed in FY10 where cerium is extracted very quickly then back extracted into the aqueous phase as the chemical equilibria that govern the lanthanide extraction proceed to completion. 


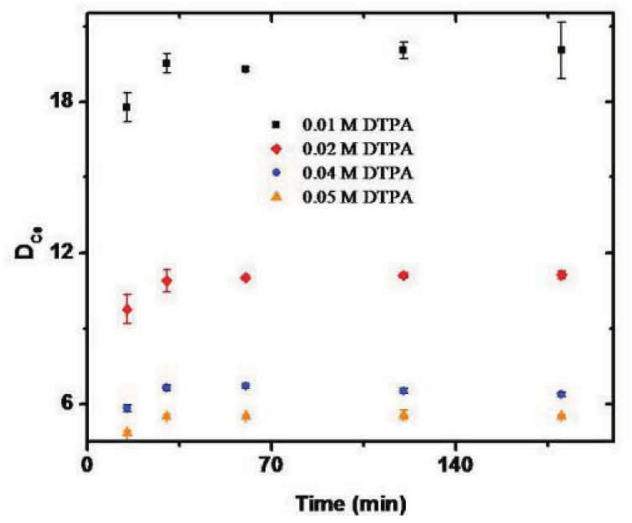

Figure 24. Influence of [DTPA $]_{(\mathrm{aq})}$ on rate of extraction of cerium by $0.17 \mathrm{M}$ HDEHP in dodecane using a rotary mixer to agitate the two phases. Aqueous phase composition $1 \mathrm{M}$ lactic acid at pH 3.8, [DTPA] 10-50 mM, [Ce] $10 \mathrm{mM},[\mathrm{Eu}] 10$ $\mathrm{mM}$.

It is not clear in these investigations if the equilibrium is reached more quickly due to the organic phase being loaded with metal ions under study. In these experiments the organic phase concentration of HDEHP is $0.17 \mathrm{M}$. However, HDEHP is known to dimerise in dodecane. This would mean that there is effectively only $0.085 \mathrm{M}$ of extractant available (Ce and $\mathrm{Eu}$ are extracted according to Equations 9 and 10 where the metal ion is extracted by three HDEHP dimers). Therefore, to completely extract all Eu and Ce from the aqueous phase would require $0.06 \mathrm{M}$ of HDEHP dimer, leaving only $0.025 \mathrm{M}$ of free HDEHP in the organic phase.

$$
\begin{gathered}
E u(D T P A)^{2-}+\overline{3(H A)_{2}} \Leftrightarrow \overline{E u\left(\mathrm{AHA}_{2}\right)_{3}}+H_{3} D T P A^{2-} \\
\operatorname{Ce}(D T P A)^{2-}+\overline{3(H A)_{2}} \Leftrightarrow \overline{\operatorname{Ce}\left(H_{2}\right)_{3}}+H_{3} D T P A^{2-}
\end{gathered}
$$

Equation 9

Equation 10

Although mathematically the organic phase is not loaded in this situation, it is entirely feasible that the organic phase has "chemically" reached the limit of metal loading. Further study is required with higher concentrations of HDEHP in the organic phase to identify the organic phase metal loading limits.

\subsubsection{Effect of HDEHP Concentration on Eu Solvent Extraction Kinetics}

To study the effects of HDEHP concentration on the Eu extraction kinetics, experiments were set up as follows:

Aqueous phase

- $1 \mathrm{M}$ total lactate $\mathrm{pH}$ adjusted to 3.6

- Varying DTPA concentration $50 \mathrm{mM}$ 
- $10 \mathrm{mM} \mathrm{Eu}$, tracer Am

Organic phase

- $\quad 0.2,0.5$ and 1.0M M HDEHP in n-dodecane

Mixing

- All organic phases were pre-equilibrated with appropriate aqueous phases

- A vortex mixer was used to contact the aqueous and organic phases for $0.5,1,1.5,2,3,6$, 10 and 20 minutes

The distribution ratios of europium as a function of mixing time and HDEHP concentration are presented in Figure 26. These data show that at all HDEHP concentrations, when using a vortex mixer for agitation, the extraction of europium reaches equilibrium between 10 and 20 mins mixing time. As the concentration of HDEHP in the organic phase is increased, the distribution of europium also increases. There was also little observed difference in the observed D value for macro europium to tracer europium.

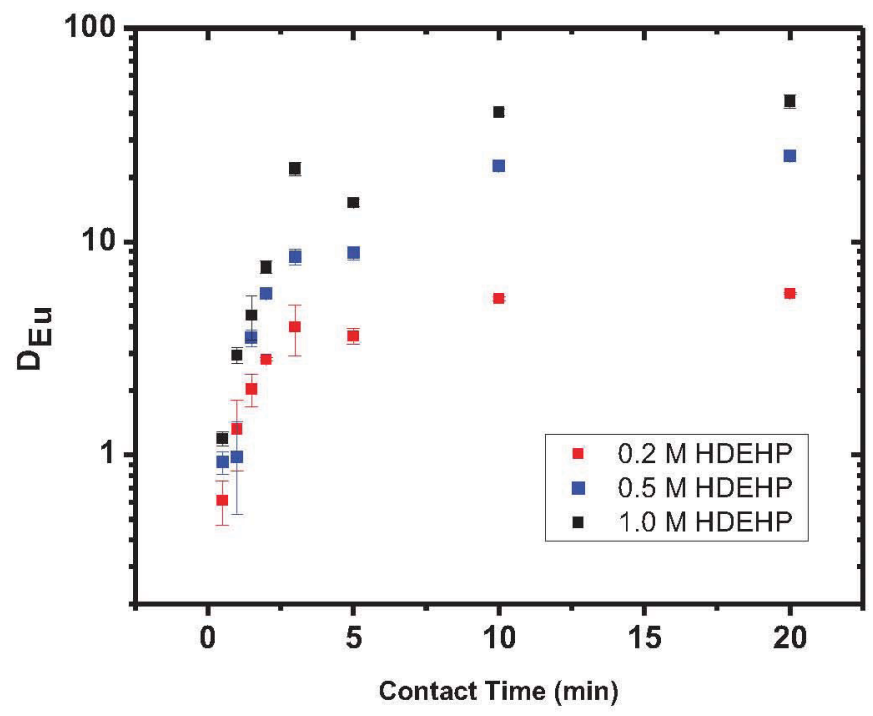

Figure 26. Influence of [HDEHP $]_{(\text {org })}$ on rate of extraction of Eu using a vortex mixer to agitate the two phases. Aqueous phase composition $1 \mathrm{M}$ lactic acid at $\mathrm{pH} 3.6,[\mathrm{DTPA}]=50 \mathrm{mM}$.

The transfer of $\mathrm{Eu}(\mathrm{III})$ from the aqueous to the organic phase (Equation 9) can be described as a reversible first order reaction with a rate constant $\mathrm{k}$ obtained from the slope of the plot of equation $11 \mathrm{vs.}$ time, where $\mathrm{D}_{f}$ is the distribution coefficient at equilibrium and $\mathrm{D}_{t}$ is the distribution coefficient at time $t$ shown in Figure 27a,b and c. [27]

$$
\log \left(\frac{D_{f}}{\left(1+D_{f}\right)\left[\left(\frac{D_{f}}{1+D_{f}}\right)-\left(\frac{D_{t}}{1+D_{t}}\right)\right]}\right)
$$


As expected, the results indicate that the kinetics of phase transfer increases as the concentration of HDEHP increases. Although measured, the kinetics of the americium extraction is not presented as the measured distribution values on this timeframe indicate americium comes to equilibrium after 3 minutes.
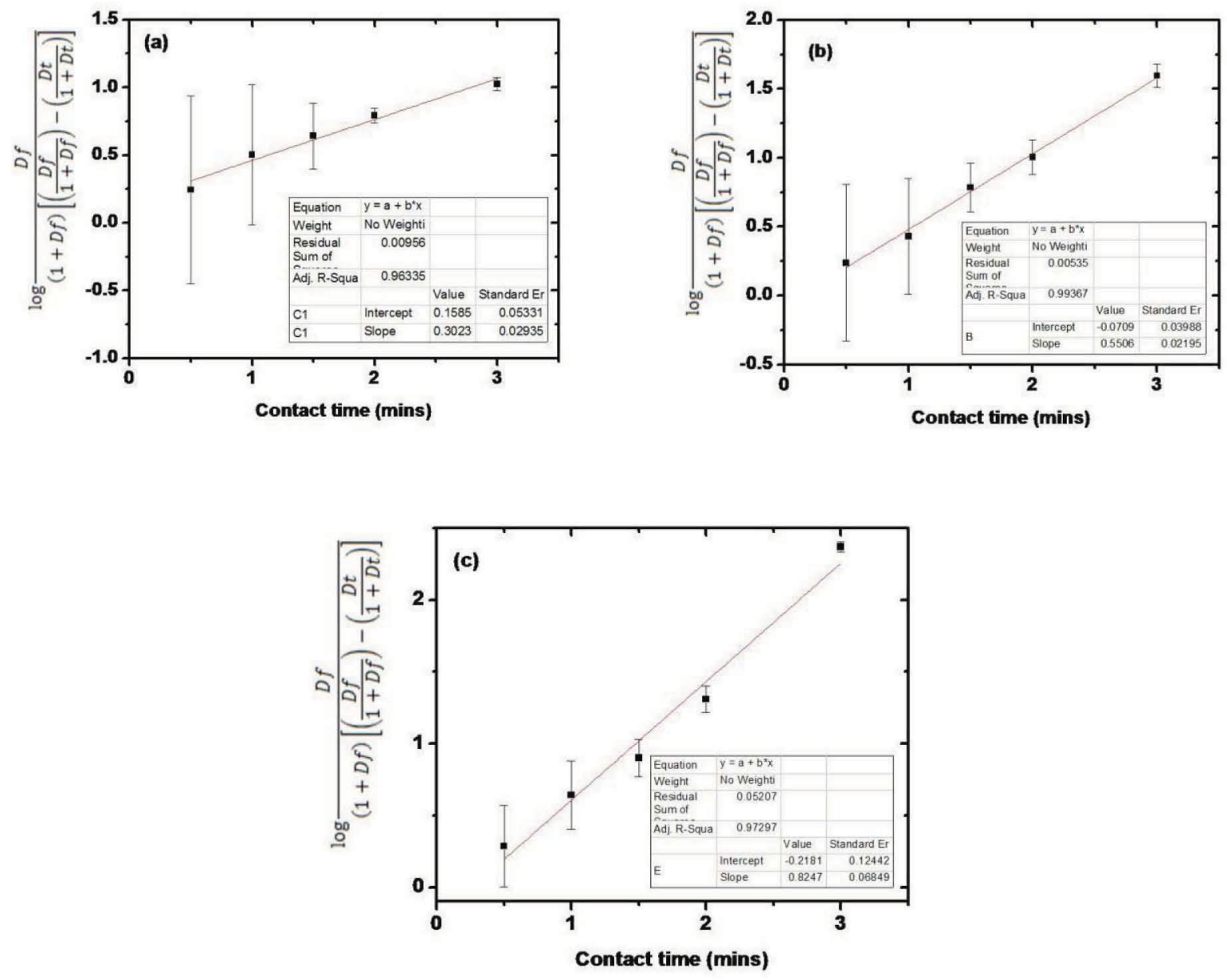

Figure27. Plots of distribution ratio as a function of Equation $11 \mathrm{vs}$ time for HDEHP concentrations of a) $0.2 \mathrm{M}$, b) $0.5 \mathrm{M}$ and c) $1.0 \mathrm{M}$. Aqueous phase composition $1 \mathrm{M}$ lactic acid at $\mathrm{pH} 3.6,[\mathrm{DTPA}]=50 \mathrm{mM}$.

Table 3 shows the individual rates determined from these experiments for each concentration of HDEHP. There is a general increase in the rate of extraction as the concentration of HDEHP is increased with only a small difference noted between 0.2M HDEHP and 0.5M HDEHP in the organic phase. This result somewhat agrees with previous studies performed by Kolarik.[27] The overall trend in $\log (\mathrm{k})$ vs $\log$ [HDEHP] follows closely with previous work having the shape $\log (y)=\log \left(1+x^{3}\right)$. In this study, the data show significantly higher rate constants than Kolarik's study, this can most likely be attributed to a more vigorous mixing speed. The slowing of the rate of extraction at lower organic phase HDEHP concentrations, is most likely explained by the increased ratio of Eu(III)-DTPA complexes in the aqueous phase to the available HDEHP in the organic phase. As the decomplexation of the europium from the DTPA is the rate determining step, and not the phase transfer itself, there could be a saturation effect on the HDEHP at the interface. It is also possible that a lamellar formation at the interface may impede the formation of dimers, resulting in slower complexation of europium and HDEHP. 
Table 3. Calculated kinetic parameters for Eu extraction at various HDEHP concentrations in the organic phase

\begin{tabular}{cc}
\hline [HDEHP] $]_{\text {org }}(\mathbf{M})$ & $\mathbf{k}\left(\mathbf{m i n}^{-1}\right)$ \\
\hline 0.2 & 0.30 \\
0.5 & 0.55 \\
1.0 & 0.82 \\
\hline
\end{tabular}

The leveling of the rate constant in the lower concentration region of this study can be explained by the fraction of Eu(III)-DTPA complexes becoming smaller with increasing HDEHP concentrations which follows a slower path. This is due to the fact that the dissociation of the Eu(III)-DTPA complex is the rate determining step, not the phase transfer itself. Kolarik ascribed this effect to a saturation of HDEHP at the interface.[27] It is also possible that a lamellar formation at the interface may impede the formation of dimers, resulting in slower complexation of europium and HDEHP.

In general, the results obtained in these studies agree well with previous work. The increase in the phase transfer kinetics of the system under study, suggests a change in aggregation state of the HDEHP molecules as concentration increases. HDEHP concentration plays a small role in the overall rate equation for the phase transfer process but it is not the rate determining step. The rate determining step is the dissociation of the Eu(III)-DTPA complex, not the phase transfer itself.

\section{Conclusions and Future Work}

The work presented in this report has demonstrated that, in TALSPEAK separations containing up to 0.1 M free lactate in solution, a classical ion-exchanging phase transfer mechanism predominates, where three HDEHP dimers solvate the metal ion in the organic phase dominates mechanism of metal extraction. This was determined using a graphical interpretation of the observed data that incorporated corrections for non-ideal behavior of HDEHP dimer in aliphatic diluent as well as sodium extraction equilibria. As the aqueous phase composition increases to high lactate concentration it is no longer possible to perform such slope analysis. Using normalized distribution ratios for the extracted lanthanides at high lactate concentrations a slope of -1 was observed. This has been attributed to either a lactate interference in the aqueous phase complexation of the lanthanide ions or the participation of a lactate molecule in the extracted complex. These findings agree with our initial hypothesis that the extraction mechanism changes as lactate concentration increases in the aqueous phase. The observed shape in the lanthanide distribution curve in our studies of TALSPEAK systems has been demonstrated to be the same for solutions containing no lactate or $2.3 \mathrm{M}$ lactate. As such it can be concluded that the mechanism of extraction is not altered dramatically but remains sensitive to effective charge density of the metal ion.

The ability to measure the heat of extraction of curium in a well defined system has been accomplished. The heat of extraction determined by isothermal titration calorimetry has been compared to a "conditional" heat of extraction determined by van't Hoff treatment of Cm distribution as a function of temperature. The two methods gave heats of extraction as $-10.0 \pm 1.0$ and $-7.21 \pm 0.38 \mathrm{~kJ} \mathrm{~mol}^{-1}$, respectively, which are in reasonable agreement with each other.

The phase transfer kinetics studies have further highlighted the difficulty of relying on tracer investigations alone to adequately describe the chemistry in liquid-liquid distributions. The research presented here has shown that metal concentration in a lactate based separations system could be critical 
in understanding the kinetics of extraction. Further, when probing the effects of HDEHP concentration in the organic phase, it has been observed that the rate of extraction increases with HDEHP concentration. As yet it is not clear if the slower extraction kinetics at low HDEHP concentrations are due to metal loading of the organic phase or that lamellar formation at the interface may impede the formation of dimers, resulting in slower complexation of europium by HDEHP.

In FY 2012 research will continue to develop thermodynamic and kinetic parameters that will assist in describing the advanced separations processes. In particular, investigations on the use of isothermal titration calorimetry to study the heat of extraction in non ion exchanging extraction systems will be studied. In addition to this, solution phase EXAFS measurements will be made to investigate the aqueous phase coordination environment around lanthanide ions. Data collection on solution phase complexation kinetics of the actinides will also continue.

\section{Indicators of Productivity and Project Quality}

N. J. Bridges, L. E. Roy, L. R. Martin "Alternative Means of Investigation TALSPEAK Process", Presented by N. J. Bridges, before $35^{\text {th }}$ Actinide Separation Conference (2010), Charlotte, NC

L. E. Roy, N. J. Bridges, L. R. Martin "Theoretical Study of the Separation of Am" from Nd" with Diethylenetriaminepentaacetic Acid" in preparation

Guoxin Tian, Leigh R. Martin., Zhang, Z. and Linfeng Rao. Thermodynamic, Spectroscopic and Computational Studies of Lanthanide Complexation with Diethylenetriaminepentaacetic acid: Temperature Effect and Coordination Modes. Inorganic Chemistry, (2011), Vol 50, pp 3087-3096.

Leigh R. Martin, Thermodynamics and Kinetics of Advanced Separations Systems, National Nuclear Laboratory, UK, May $10^{\text {th }}$ 2011. Invited Talk.

Tamara L. Griffiths, Clint Sharrad, Louise Natrajan, Georgina Roughley, Mark Sarsfield, Peter R. Zalupski and Leigh R. Martin. Investigations of ternary actinide complexes relevant to the nuclear fuel cycle. $35^{\text {th }}$ Actinide Separations Conference, Charlotte, NC, May $23^{\text {rd }}-27^{\text {th }} 2011$.

Richard D. Tillotson, Peter R. Zalupski and Leigh R. Martin,. The role of DTPA in the unusual extraction kinetics of TALSPEAK. $35^{\text {th }}$ Actinide Separations Conference, Charlotte, NC, May $23^{\text {rd }}$ $-27^{\text {th }} 2011$.

Leigh R. Martin, Thermodynamics and Kinetics of Advanced Separations Systems, Invited Poster, 2011 Separations and Heavy Element Chemistry Research Meeting, Baltimore, MD, April 26-29, 2011.

Tamara L. Griffiths, Clint Sharrad, Louise Natrajan, Georgina Roughley, Mark Sarsfield, Peter R. Zalupski and Leigh R. Martin. Investigations of ternary actinide complexes relevant to the nuclear fuel cycle. Abstracts of Papers, $241^{\text {st }}$ ACS National Meeting, Anaheim, CA, United States, March 27-31, 2011.

Peter R. Zalupski and Leigh R. Martin, Studying the less-Predictable Realms of TALSPEAK Chemistry, OECD Nuclear Energy Agency (NEA) Actinide and Fission Product Partitioning and Transmutation $11^{\text {th }}$ Information Exchange Meeting, San Francisco., United States, November 1-4, 2010. 


\section{Collaborators and Participants}

1. Leigh R. Martin, Idaho National Laboratory (PI)

2. Peter R. Zalupski, Idaho National Laboratory, co-researcher

3. Travis S. Grimes, Idaho National Laboratory, Post doctoral researcher

4. Richard D. Tillotson, Idaho National Laboratory.

5. Linfeng Rao, Lawrence Berkeley National Laboratory.

6. Guoxin Tian, Lawrence Berkeley National Laboratory.

7. Nicholas Bridges, Savannah River National Laboratory.

8. Tracy Rudisil, Savannah River National Laboratory.

9. Artem Guelis, Argonne National Laboratory.

10. Clint Sharrad, University of Manchester.

11. Louise Natrajan, University of Manchester.

12. Tamara Griffiths, University of Manchester.

13. Stephen P. Mezyk, Cal State Long Beach.

14. Keri Campbell, student, University of Nevada Las Vegas

15. Joseph Lapka, student, University of Oregon

\section{References Cited}

1. Cox, M. and Rydberg, J.: Introduction to Solvent Extraction. In Solvent Extraction Principles and Practice $2{ }^{\text {nd }}$ Edition, Rydberg, J.Cox, M. and Choppin, G.R. (eds), pp-1-25. Marcel Dekker Inc., New York, USA (2004).

2. Gannaz, B., Chiarizia, R.; Antonio, M. R.; Hill, C. and Cote, G. Solv. Extr. Ion Exch., 25, 313 (2007).

3. Hill, C., Guillaneux, D., Berthon, L. and Madic, C. J. Nucl. Sci. Technol. 309, (2002).

4. Magnusson, D., Christiansen, B., Foreman, M. R. S., Geist, A., Glatz, J. P., Malmbeck, R., Modolo, G., Serrano-Purroy, D. and Sorel, C, Solv. Extr. Ion Exch. 27, 97, (2009).

5. Weaver, B; Kappelmann, F., TALSPEAK, A new method of separating americium and curium from the lanthanides by extraction from an aqueous solution of an aminopolyacetic acid complex with a monoacetic organophosphate or phosphonate. ORNL-3559, (1964).

6. Nilsson, M. and Nash, K.L., Solv. Extr. Ion Exch., 25, 665 (2007).

7. Nilsson, M. and Nash, K.L., Solv. Extr. Ion Exch., 27, 354 (2009).

8. Martin, L.R., Mincher, B.J. and Schmitt, J. Radioanal. Nucl. Chem., 282, 523 (2009).

9. Martin, L.R. and Zalupski, P.R., Thermodynamics and Kinetics of Advanced Separations Systems - FY2010 Summary Report. INL Report FCRD-SEPA-2010-000174.

10. Danesi, P.R. and Vandergrift, G.F. . Inorg. Nucl. Chem. Letters 17, 109 (1981).

11. Danesi, P.R., Cianetti, C. and Horwitz, E.P. Sep. Sci. Technol. 17, 507 (1982).

12. McDowell, W.J. and Coleman, C.P. J. Inorg. Nucl. Chem. 27, 1117 (1965).

13. Grimes, T., Nilsson, M. and Nash, K.L. Sep. Sci. Technol. 45, 1725 (2010).

14. Leggett, C.J., Guokui, L. and Jensen, M.P. Solvent Extr. Ion Exch. 28, 313 (2010).

15. Kosyakov, V.N. and Yerin, E.A. J. Radioanal. Chem. 56, 93 (1980).

16. Weaver, B. and Kappelmann, F.A. J. Inorg. Nucl. Chem. 30, 263(1968).

17. Choppin G.R., J. Alloys Comp. 344, 55 (2002).

18. Choppin G.R., J. Alloys Comp. 223, 174-179 (1995).

19. Peppard D.F., Bloomquist C.A.A., Horwitz E.P., Levey, S. and Mason G.W., J. Inorg. Nucl. Chem. 32, 339 (1970).

20. Martell AE, Smith RM NIST Critically Selected Stability Constants of Metal Complexes Database; Version 8.0. (2004)

21. Shannon, R.D., Acta Cryst. A32, 751 (1976) 
22. Kolarik Z (1971) Interactions of acidic organophosphorus extractants in the organic phase. In: Marcus Y (ed) Solvent extraction reviews. Marcel Dekker, New York, pp. 1-62.

23. Kolarik, Z. and Pankova, H., J. Inorg. Nucl. Chem. 28, 2325 (1966).

24. Mason G.W, Lewey S. and Peppard D.F., J. Inorg. Nucl. Chem. 26, 2271 (1964).

25. Kalina, D.G., Mason, G.W. and Horwitz, E.P., J. Inorg. Nucl. Chem. 43, 579 (1980)

26. Zalupski, P.R. and Nash, K.L.: Solv. Extr. Ion Exch. 26, 514-533 (2008).

27. Kolarik, Z. and Kuhn, W., Acidic Organophosphorus Extractants - XXI. Kinetics and Equilibria of Extraction of Eu(III) by Di(2-ethylhexyl) Phosphoric Acid From Complexing Media. Proceeding International Solvent Extraction Conference Vol. 3, 2593 (1974).

\section{Summary of Collaborators Efforts in FY2011}

The thermodynamics and kinetics program is a multi national laboratory effort for the development of the necessary expertise and capabilities in actinide solution chemistry that will assist in describing the fundamental chemistry that drives advanced aqueous separations processes. Although the current focus is primarily towards trivalent actinide/ lanthanide separations, the expertise and capabilities are being developed in such a manner that they could be deployed to any separations technique proposed.

Each lab has their own main focus area, with some overlap into the INL program for consistency in parameter measurements. The PI's at LBNL, SRNL and ANL all produce their own end of year report for its focus area; however in this section the main highlights of the research from each contributor is summarized to show how this program is developing. Detailed reports from each contributor are presented in Appendix A-D.

\subsection{LBNL}

At Lawrence Berkeley National Laboratory (LBNL) the team lead by Dr. Linfeng Rao focuses on the determination of thermodynamic parameters of aqueous complexes between $\mathrm{Ln}(\mathrm{III}) / \mathrm{An}$ (III) and TALSPEAK related ligands. These studies are designed to assist our understanding of the actual speciation in the complex aqueous phase of TALSPEAK. The techniques applied for these studies are potentiometry, absorption spectrophotometry, luminescence spectrophotometry and calorimetry. In FY11, the focus has been on the coordination modes of DTPA with lanthanides ( $\mathrm{Nd}$ and Eu) in solution, and studying the spectroscopic properties of $\mathrm{Cm}$ aqua ion as a function of temperature.

\subsubsection{Summary of LBNL results}

Spectroscopic data on the complexation of DTPA to Eu and Nd have been compared with DFT calculations in an attempt to identify the coordination mode of HDTPA ${ }^{4-}$ to these metal ions in aqueous solution. By using both theory and experiment it has been determined that the HDTPA ${ }^{4-}$ metal complex in aqueous solution remains octa-dentate like the fully deprotonated DTPA ${ }^{5-}$ metal complex is in solution.

The fluorescent lifetime of $\mathrm{Cm}$ (III) has been studied over the range $10-85^{\circ} \mathrm{C}$. Reliable information on the quantum yield and luminescence lifetime of curium in aqueous solution has been obtained and a correlation between the hydration number and luminescence lifetime has been developed. This correlation will be utilized in subsequent studies to determining the hydration number for $\mathrm{Cm}$ when it is bound to TALSPEAK ligands. 


\subsection{ANL}

At Argonne Nationional Laboratory (ANL) the team lead by Dr. Artem Guelis has focused on the use of microfluidic devices for the study of phase transfer kinetics. Kinetic data can be collected to allow for the calculation of metal diffusion coefficients since the surface area of the interface can be precisely calculated. In FY 11 the kinetics of extraction of the lanthanides in a citrate based TALSPEAK separation have been studied.

\subsubsection{Summary of ANL results}

A new device has been produced to study the kinetics of extraction of the lanthanide elements in a citrate buffered TALSPEAK separation. The majority of the experiments were performed using a device that was comprised of an $88 \mathrm{~mm}$ tube with an internal diameter of $126 \mu \mathrm{m}$; the flow rates were varied to obtain different residence times in the tubing. The concentration of the lanthanides were determined by ICP-MS. The initial metal concentration was either $10 \mathrm{mM}$ or $1 \mathrm{mM}$.

The results obtained from this study identified that the rate of extraction of the lanthanide elements decreases from lanthanum to dysprosium. The rate of extraction is then seen to increase again out to lutetium. This kinetics effect has not been previously observed for the heavier lanthanides. It has been suggested that the buffer in the separation system may play a large role in determining the rate of extraction in TALSPEAK-type systems. Some tests were performed to investigate the effects of metal interference in the kinetics of extraction but no evidence of this was observed under the conditions studied.

\subsection{SRNL}

At Savannah River Nationional Laboratory (SRNL) there are two teams investigating slightly different aspects of TALSPEAK chemistry. The effort lead by Dr. Nicholas Bridges uses electrochemistry and computational studies to provide a new approach to understanding metal-ligand interactions between the actinides and TALSPEAK relevant ligands. Dr. Tracy Rudisill has been investigating the therochemical behavior of other actinides within TALSPEAK separations.

\subsubsection{Electrochemical and Computation Investigation of TALSPEAK Chemistry}

Complexation of $\mathrm{Pu}$ in both the +3 and +4 oxidation state has been studied using electrochemical methods and UV-visible spectroscopy. Although there are several free DTPA species present in solution in the $\mathrm{pH}$ range studied, it was identified that for both $\mathrm{Pu}^{+3}$ and $\mathrm{Pu}^{+4}$ there was only a $1: 1 \mathrm{Pu}$ :DTPA complex present in solution. It has been identified that when DTPA is complexed to Pu there is a significant shift in the potential of the $\mathrm{Pu}^{\mathrm{IIIV}}$ couple, as such the measured potential can be used to evaluate the ratio of the $\log \beta$ of Pu-DTPA (for both the +3 and +4 ions) in the TALSPEAK pH range. Finally, it was observed that $\mathrm{Pu}(\mathrm{IV})$ can be reduced to Pu(III) when complexed by DTPA using electrochemical methods, this is of note as in FY10 it was observed that once Pu(IV) was complexed by DTPA chemical methods could not be used to reduce it.

Molecular modeling has identified that Am complexation with DTPA is preferred over Nd by $\sim 1 \mathrm{kcal}$ $\mathrm{mol}^{-1}$. The preference results from a combination of subtle effects including the larger radial extent of the $5 f$ orbitals in causing increased covalency with preference for the oxygen chelates. This interpretation has been supported by Mulliken population and AIM analysis. In addition, a small amount of covalent behavior between the $\mathrm{M}-\mathrm{N}$ bonds also allows for additional stabilization of the americium complex. 


\subsubsection{Solvent extraction}

The stability of $\mathrm{Pu}(\mathrm{III})$ and $\mathrm{Np}(\mathrm{V})$ in TALSPEAK buffer media has been studied using time-dependent UV-vis spectrophotometry. It has been identified that the Pu(IV)-DTPA and Np(IV)-DTPA complexes are the most stable in $1 \mathrm{M}$ lactate solution. It was found in these studies that the oxidation of plutonium was significantly faster than the reduction process of the neptunium. This is not necessarily unexpected as the neptunium reduction process requires the neptunyl $\mathrm{Np}=\mathrm{O}$ bonds to be broken, whereas the oxidation of plutonium is a simple one electron transfer process.

Distribution measurements of $\mathrm{Np}$ (IV), Pu(IV) and Am(III) into 1.0 M HDEHP from $50 \mathrm{mM}$ DTPA in $1 \mathrm{M}$ lactic acid at $\mathrm{pH} 3.5$ have been measured as a function of temperature. The actinides in the +4 oxidation state were less well extracted into the organic phase than americium, this was attributed to the stronger interaction between the +4 ions and the DTPA complexant in the aqueous phase. The conditional enthalpies of extraction of $\mathrm{Np}$ (IV) and $\mathrm{Pu}$ (IV) were calculated by van't Hoff analysis and identified as $-12.5 \pm 3.3$ and $1.3 \pm 1.1 \mathrm{~kJ} \mathrm{~mol}^{-1}$ respectively. 


\title{
Appendix A
}

\section{Understanding Actinide/Lanthanide Speciation under TALSPEAK Conditions}

\author{
Dr. Linfeng Rao
}

Lawrence Berkeley National Laboratory 


\section{Fuel Cycle Research and Development Separations UNDERSTANDING ACTINIDE/LANTHANIDE SPECIATION UNDER TALSPEAK CONDITIONS}

\section{INTRODUCTION}

To achieve accurate prediction and precise control of the behavior of actinides and lanthanides in the TALSPEAK process, we have started systematic studies to determine the thermodynamic parameters of the reactions in the TALSPEAK system, including the complexation of lanthanides and actinides with key TALSPEAK ligands, including lactate, DTPA and nitrate. The effect of operating conditions (e.g. temperature) on the thermodynamic parameters is evaluated. In FY11, the focus has been on the coordination modes and structure of Ln(III)/DTPA complexes, and the spectroscopic properties and hydration of $\mathrm{Cm}$ (III) ion in aqueous solutions.

\section{RESULTS}

\subsection{Complexation of $\mathrm{Nd}(\mathrm{III})$ and $\mathrm{Eu}(\mathrm{III})$ with DTPA $(10-70 \circ \mathrm{C})-$ Coordination Modes and Structure of Ln(HDTPA)- Complex}

In the studies conducted in FY10, stability constants of the Eu(III)/DTPA complexes at $10-70$ 。C were determined by analyzing the luminescence emission spectra obtained. Representative spectra at $25 \circ \mathrm{C}$ are shown in Figure 1. To reveal the coordination modes and the structure of the lanthanide DTPA complexes, the work in FY 11 was focused on (1) the determination of the hydration numbers of Eu(III) in the DTPA complexes by luminescence lifetime measurements, and (2) identification of the optimal structure(s) of Ln/DTPA complexes by Density Functional Theory (DFT) calculations.

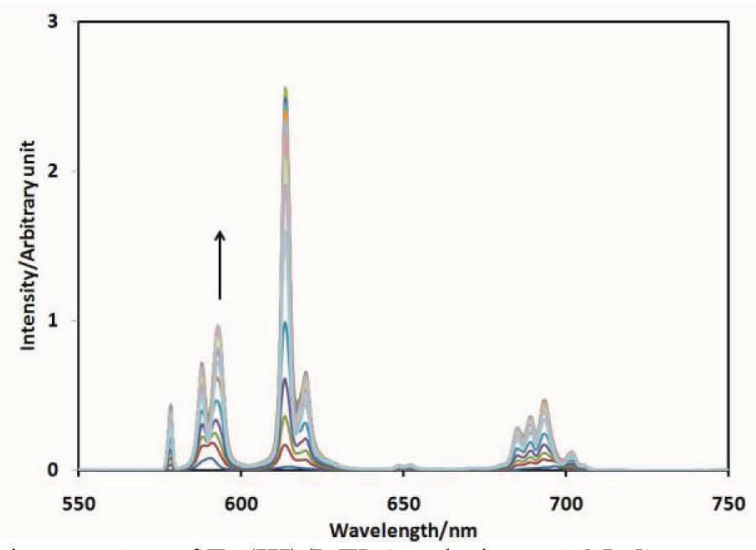

Figure 1. Luminescence emission spectra of Eu(III)/DTPA solutions at 25。C.

\subsubsection{The hydration number of Eu(III) in the DTPA complexes: measurement of luminescence lifetime for the Eu(III)/DTPA system $(10-70$ 。C)}

The luminescence lifetime of $\mathrm{Eu}(\mathrm{III})$ in aqueous solutions ([Eu(III)] $=2 \mathrm{mM}$, [DTPA] $=0-3.3 \mathrm{mM}$ ) were measured on a HORIBA Jobin Yvon IBH FluoroLog-3 fluorometer adapted for time-resolved 
measurements. $10 \mathrm{~mm}$ quartz cells were used. A sub-microsecond Xenon flash lamp (Jobin Yvon, $5000 \mathrm{XeF}$ ) was the light source and coupled to a double grating excitation monochromator for spectral selection. The input pulse energy ( $100 \mathrm{nF}$ discharge capacitance) was about $50 \mathrm{~mJ}$ and the optical pulse duration was less than $300 \mathrm{~ns}$ at fwhm. A thermoelectrically cooled single photon detection module (HORIBA Jobin Yvon IBH, TBX-04-D) that incorporates a fast rise time PMT, a wide bandwidth preamplifier, and a picosecond constant fraction discriminator was used as the detector. Signals were acquired using an IBH Data Station Hub and data were analyzed using the commercially available DAS 6 decay analysis software package from HORIBA Jobin Yvon IBH. The goodness of fit was assessed by minimizing the reduced function, $\chi_{2}$, and visually inspecting the weighted residuals. Each trace contained at least 10,000 points, and the reported lifetime values resulted from at least three independent measurements.

Figure 2 shows the luminescence decay of Eu(III)/DTPA solutions at $25_{\circ} \mathrm{C}$. It is evident that, as the concentration of DTPA is increased (from curves 1 to 17), the luminescence lifetime becomes longer. Single exponential functions were used to fit the data to obtain the luminescence lifetimes $(\tau)$, and the data are shown in Table 1.

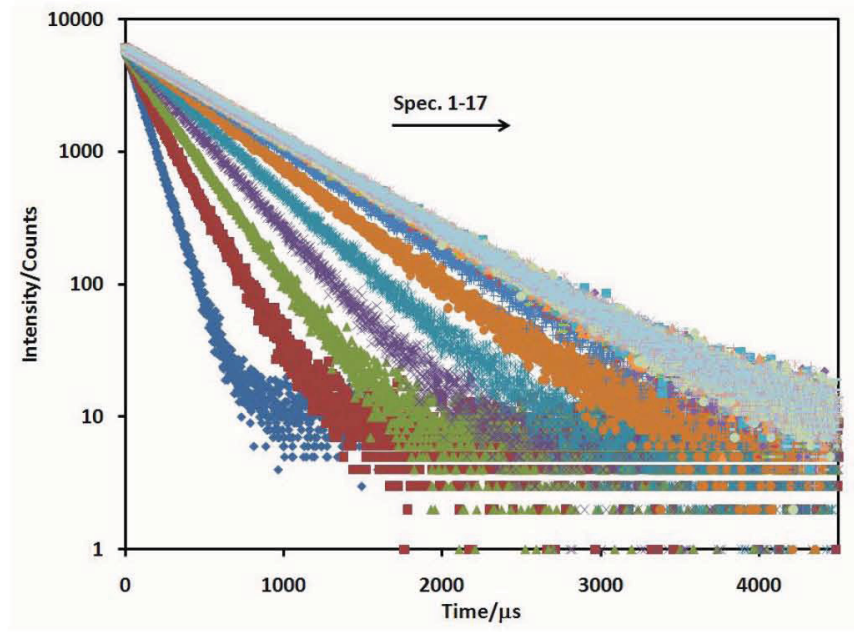

Figure 2. Luminescence decay of $\mathrm{Eu}(\mathrm{III}) / \mathrm{DTPA}$ solutions, from curves 1 to 17 , the ratio of $\mathrm{CDTPA}_{\mathrm{DE}} \mathrm{CEu}_{\text {increases }}$ from 0 to 3 .

It is known that the quenching of the luminescence of Eu(III) in aqueous solutions is due to the efficient energy transfer from the excited state $(5 \mathrm{D} 0)$ of $\mathrm{Eu}$ (III) to the $\mathrm{O}-\mathrm{HI}$ vibration of the coordinated water molecules. Therefore, there is a correlation between the luminescence lifetime and the hydration number of Eu(III) in the inner coordination sphere [1]. Using the lifetime data from this work (Table 1) and the previously established linear correlation between the luminescence lifetime and the hydration number of $\mathrm{Eu}$ (III) $\left(n \mathrm{H}_{20}=1.05 \times \tau_{-1}-0.7\right.$, where $\tau$ is in milliseconds [2]), the hydration number per Eu(III) in the solution, $n$ H2O,exp, was determined (Table 1). The gradual decrease in the hydration number per $\mathrm{Eu}$ (III) with the increase in the concentration of DTPA, as shown in Table 1, indicates that the water molecules in the inner coordination sphere of Eu3 + is gradually replaced by DTPA. 


\begin{tabular}{|c|c|c|c|c|c|c|c|c|}
\hline \multirow[b]{2}{*}{$C_{\mathrm{L}} / C_{\mathrm{En}}$} & \multirow[b]{2}{*}{$\tau_{\mathrm{exp}} \mu \mathrm{S}$} & \multirow{2}{*}{$\begin{array}{c}n_{\mathrm{H} 20} \\
\exp \\
( \pm 0.5)\end{array}$} & \multicolumn{3}{|c|}{$\%$ Eu species } & \multicolumn{3}{|c|}{$n_{\text {HzO }}$ cal } \\
\hline & & & $\mathrm{Eu}^{3+}$ & $\mathrm{EuL}^{2-}$ & $\mathrm{Eu}(\mathrm{HL})^{-}$ & $\begin{array}{c}\text { Assuming } \\
\mathrm{Eu}(\mathrm{HL}) \cdot 3 \mathrm{H}_{2} \mathrm{O}\end{array}$ & $\begin{array}{c}\text { Assuming } \\
\mathrm{Eu}(\mathrm{HL}) \cdot 1.5 \mathrm{H}_{2} \mathrm{O}\end{array}$ & $\begin{array}{c}\text { Assuming } \\
\mathrm{Eu}(\mathrm{HL}) \cdot \mathrm{H}_{2} \mathrm{O}\end{array}$ \\
\hline 1.118 & 560.0 & 1.33 & 2.8 & 34.7 & 62.6 & 2.47 & 1.53 & 1.22 \\
\hline 1.304 & 601.0 & 1.20 & 0.5 & 41.6 & 57.9 & 2.20 & 1.33 & 1.04 \\
\hline 1.491 & 616.0 & 1.15 & 0.1 & 49.5 & 50.4 & 2.02 & 1.26 & 1.01 \\
\hline 1.677 & 626.0 & 1.13 & 0 & 57.4 & 42.5 & 1.85 & 1.21 & 1.00 \\
\hline 1.863 & 629.0 & 1.12 & 0 & 64.6 & 35.4 & 1.71 & 1.18 & 1.00 \\
\hline 2.050 & 633.0 & 1.11 & 0 & 70.8 & 29.2 & 1.58 & 1.15 & 1.00 \\
\hline 2.236 & 633.0 & 1.11 & 0 & 76.1 & 23.9 & 1.48 & 1.12 & 1.00 \\
\hline 2.422 & 635.0 & 1.10 & 0 & 80.6 & 19.4 & 1.39 & 1.10 & 1.00 \\
\hline 2.609 & 638.0 & 1.10 & 0 & 84.4 & 15.6 & 1.31 & 1.08 & 1.00 \\
\hline 2.795 & 639.0 & 1.09 & 0 & 87.6 & 12.4 & 1.25 & 1.06 & 1.00 \\
\hline 2.981 & 638.4 & 1.09 & 0 & 90.3 & 9.7 & 1.19 & 1.05 & 1.00 \\
\hline
\end{tabular}

The values of $n \mathrm{H} 2 \mathrm{O}$,exp represent the average hydration number of all Eu(III) species in solution, including free $\mathrm{Eu}_{3+}$, EuL2- and EuHL-. Of these species, the free $\mathrm{Eu}_{3}+$ is known to have 9 water molecules in the inner coordination sphere $\left(n \mathrm{H}_{20}=9\right)[2,3]$. The 1:1 Eu(III)/DTPA complex, EuL2-, has been shown to be an octa-dentate chelate complex in which the DTPA coordinates to $\mathrm{Eu(III)}$ with five carboxylate oxygen atoms and three amine nitrogen atoms, leaving one position for a water molecule ( $n \mathrm{H} 2 \mathrm{O}=1)$ [3-7]. In contrast, the hydration number in the protonated Eu(III)/DTPA complex, EuHL-, remains uncertain. A hexa-dentate chelate complex for LaHL- or YbHL-, implying $n \mathrm{H} 2 \mathrm{O}=3$, has previously been suggested but not substantiated with experimental evidence [8]. The luminescence lifetime data in conjunction with the DFT calculations in this work, as discussed below, could help to clarify the coordination mode and hydration number of the protonated Eu(III)/DTPA complex.

\subsubsection{The structure of Eu(HDTPA)-Complex: DFT Calculations}

Geometry optimization of the structures of EuL2- and EuHL- complexes (where L stands for DTPA) was performed by DFT calculations. The optimized structures and the bond lengths are shown in Figure 3 and Table 2, respectively. The structure of EuL2- (Figure 3a) is optimized from the crystallographic data for this complex in the literature without the counter ions [7]. Using the optimized structure of EuL2- as a starting point, the structures of EuHL-were further optimized by placing the proton on either the nitrogen of one amine group (Figure $3 b$ ) or the oxygen of one carboxylate group (Figure $3 c$ ). 


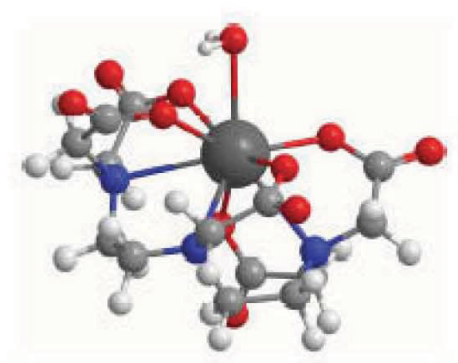

(a)

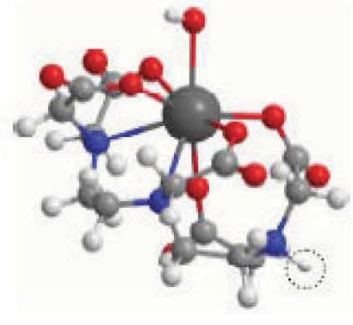

(b)

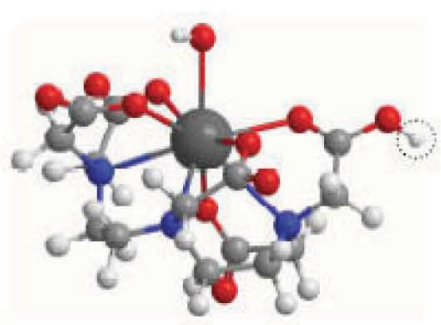

(c)

Figure 3. Optimized structures of the octa-dentate Eu(DTPA) $)^{2-}$ complex (a), the protonated $\mathrm{Eu}(\mathrm{HDTPA})^{-}$complex where the protonation occurs on the amine nitrogen (b), and the protonated $\mathrm{Eu}(\mathrm{HDTPA})^{-}$complex where the protonation occurs on the carboxylate oxygen (c). Black - Eu, red oxygen. Blue - nitrogen, gray - carbon, white - hydrogen. The position of protonation is indicated with a dashed circle. The energy of structure (b) is higher than that of the structure (c) by about $0.62 \mathrm{eV}$.

Table 2 Calculated Eu-N and Eu-O bond lengths $(\AA)$ in the Eu(III)/DTPA complexes

\begin{tabular}{lccc}
\hline & $\begin{array}{c}\text { EuDTPA }^{-2} \\
\text { (Figure 7a) }\end{array}$ & $\begin{array}{c}\text { EuH(DTPA) with } \mathrm{H} \text { on N } \\
\text { (Figure 7b) }\end{array}$ & $\begin{array}{c}\text { EuH(DTPA) with H on O } \\
\text { (Figure 7c) }\end{array}$ \\
\hline Eu-N1 & 2.893 & 4.129 & 3.042 \\
Eu-N2 & 2.761 & 2.917 & 2.709 \\
Eu-N3 & 2.873 & 2.747 & 2.734 \\
Eu-O1 & 2.365 & 2.435 & 2.616 \\
Eu-O2 & 2.435 & 2.357 & 2.382 \\
Eu-O3 & 2.402 & 2.381 & 2.370 \\
Eu-O4 & 2.505 & 2.401 & 2.402 \\
Eu-O5 & 2.448 & 2.389 & 2.403 \\
Eu-O6 & 2.673 & 2.530 & 2.550 \\
\hline
\end{tabular}

In the structure of EuL2, the DTPA ligand is octa-dentate with five oxygen atoms from the carboxylate groups and three nitrogen atoms from the amine groups coordinating to $\mathrm{Eu}(\mathrm{III})$. One water molecule is in the inner coordination sphere of $\mathrm{Eu}(\mathrm{III})$, satisfying the total coordination number of 9 (Figure $3 \mathrm{a}$ ). The oxygen atoms from the carboxylate groups and water are considerably closer to the central Eu atom (REu-O $=2.365$ to $2.673 \AA$ ) than the nitrogen atoms from the amine groups $\left(R_{\mathrm{Eu}-\mathrm{N}}=2.761\right.$ to $2.893 \AA$ ).

In the optimized structure of EuHL- with the proton on the nitrogen of one amine group (Figure $3 \mathrm{~b}$ ), the nitrogen atom moves away from the Eu atom with $R_{\mathrm{Eu}-\mathrm{N} \mathrm{l}}=4.129 \AA$. This is understandable because the basicity of this amine group would be greatly reduced upon protonation and amine coordination to $\mathrm{Eu}(\mathrm{III})$ becomes impossible. Therefore, the EuHL- complex can be described essentially as being hepta-dentate with DTPA. The hydration number of the hepta-dentate EuHL-complex could remain to be $n$ H2O $=1$, because the Eu(III) is well-"enveloped" by the DTPA ligand and is not readily accessed by additional water molecules.

If the protonation occurs on the oxygen of one carboxylate group (Figure 3c), considerable changes in some of the Eu-O and Eu-N distances are observed in the optimized structure of EuHL-. Most notably, the Eu-O1 and Eu-N1 distances become longer ( $R$ Eu-O1 from 2.365 to $2.616 \AA, R$ Eu-N1 from 2.893 to 3.042 ), indicating significant weakening of these two coordination bonds. However, all three nitrogen atoms and five oxygen atoms remain coordinated with Eu, the DTPA ligand is still octa-dentate in the EuHLcomplex, with the hydration number $n$ н $20=1$.

Besides optimizing the structures of the EuHL-complex, DFT calculations in this work also show that the energy of the EuHL-complex with the proton on the amine nitrogen (Figure $3 b$ ) is higher than that of the EuHL-complex with the proton on the carboxylate oxygen (Figure $3 \mathrm{c}$ ) by about $0.62 \mathrm{eV}$. Thus the latter, 
in which an octa-dentate ligand (DTPA) and a water molecule coordinating with $\mathrm{Eu}(\mathrm{III})$, is the more probably structure of the EuHL- complex.

By assuming the EuHL- complex has a hydration number of 1.0 as the DFT calculation shows, or 3.0 as a previous study [8] suggests, the average hydration number of $\mathrm{Eu}(\mathrm{III})$ in the $\mathrm{Eu}(\mathrm{III}) / \mathrm{DTPA}$ solutions could be obtained by speciation calculation using the stability constants from this work. The speciation of $\mathrm{Eu}(\mathrm{III})$ and the calculated $n \mathrm{H} 2 \mathrm{O}$, cal are shown in Table 2. The values of $n \mathrm{H} 2 \mathrm{O}$, cal are compared with those of $n \mathrm{H} 2 \mathrm{O}$,exp determined by luminescence decay in Figure 4. It is evident that the experimental data do not support the formation of a hexa-dentate EuHL- complex with $n \mathrm{H} 2 \mathrm{O}=3$ suggested in the literature [8]. The assumption of a EuHL- complex with $n \mathrm{H} 2 \mathrm{O}=1$, based on the results of DFT calculations, provides reasonably good agreement with the experimental data.

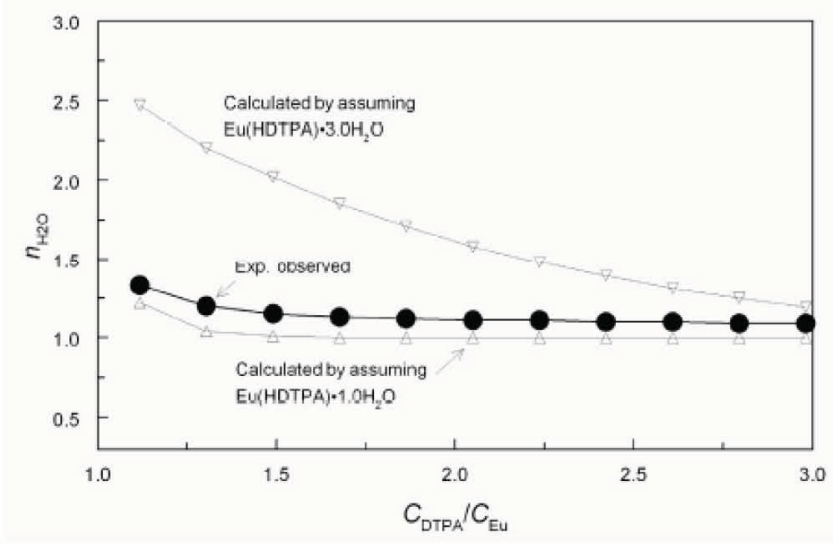

Figure 4. Comparison between the average hydration number determined by luminescence decay, $n_{\text {Ho }}$ axp and the average hydration number calculated from speciation, $n_{\text {H2O,cal. }}$

In summary, for the first time, integration of the experimental data from luminescence spectroscopy and the results of DFT calculations has lead to the conclusion that the DTPA ligand in the MHL-complex is probably octa-dentate with one protonated carboxylate group and one water molecule in the inner coordination sphere.

\subsection{Spectroscopic Properties and Hydration of the $\mathrm{Cm}$ (III) Aqua Ion from 10 to 85 。C}

After completing the studies of the complexation between $\mathrm{Ln}(\mathrm{III})$ (Nd and Eu) with TALSPEAK ligands (lactic acid and DTPA), our focus was on the complexation of Cm(III) with these ligands at different temperatures. In order to understand the coordination modes and structure of the $\mathrm{Cm}$ (III) complexes at different temperatures using the luminescence spectroscopy, it is necessary to know the correlation between the luminescence life time of $\mathrm{Cm}$ (III) and the number of water molecules in the first primary hydration sphere at different temperatures. In the literature, only one empirical correlation at $25 \mathrm{oC}$ is available [9]. Therefore, before we study the Cm(III) complexation with the TALSPEAK ligands, we must first study the spectroscopic properties and hydration of $\mathrm{Cm}$ (III) in water at different temperatures. The luminescence properties of aqua $\mathrm{Cm}$ (III) complex in $\mathrm{H}_{2} \mathrm{O}-\mathrm{D}_{2} \mathrm{O}$ systems were studied in the temperature range of 10 to $85^{\circ} \mathrm{C}$. The absorption and excitation spectra of aqua $\mathrm{Cm}$ (III) in $0.001 \mathrm{M}$ $\mathrm{HClO}_{4}$ were measured at six different carefully controlled temperatures in this range. The luminescence lifetimes and emission spectra were also measured in varying $\mathrm{H}_{2} \mathrm{O}-\mathrm{D}_{2} \mathrm{O}$ mixtures in order to determine the correlation between the lifetime and the hydration number of $\mathrm{Cm}$ (III) at different temperatures. Also, 
for the first time, the luminescence quantum yield $\Phi$ of the $\mathrm{Cm}$ (III) aqua ion was measured, and the radiative decay constants of the excited $6 \mathrm{D}^{\prime} 7 / 2$ multiplet of $\mathrm{Cm}(\mathrm{III})$ were obtained as a function of temperature. A very weak temperature dependence was observed for the non-radiative decay rates in the 10 to $85^{\circ} \mathrm{C}$ temperature range from lifetime measurements of $\mathrm{Cm}$ (III) in the $\mathrm{H}_{2} \mathrm{O}-\mathrm{D}_{2} \mathrm{O}$ system after corrections for the measured radiative decay rate. An Arrhenius type temperature-dependent quenching mechanism of the luminescence of $\mathrm{Cm}$ (III) is proposed.

\subsubsection{The excitation and emission spectra of $\mathrm{Cm}(\mathrm{III})$ in $\mathrm{H}_{2} \mathrm{O}$ and $\mathrm{D}_{2} \mathrm{O}$ at different temperatures}

The absorption spectrum of $\mathrm{Cm}(\mathrm{III})$ in $0.001 \mathrm{M} \mathrm{DClO}_{4}$ in $\mathrm{D}_{2} \mathrm{O}(>99.9 \%)$ at $25^{\circ} \mathrm{C}$ is identical to the spectra of $\mathrm{Cm}(\mathrm{III})$ in $0.1 \mathrm{M}$ and $1 \mathrm{M} \mathrm{HClO}_{4}$, indicating there is no $\mathrm{H} / \mathrm{D}$ isotope effect on the absorption of $\mathrm{Cm}$ (III) aqua ion, and the perchlorate anion is not involved in the inner coordination sphere of $\mathrm{Cm}$ (III) in the $\mathrm{ClO}_{4}$ in this concentration range. The positions of all absorption bands in UV-visible region remain unchanged as the temperature varies, but the absorbance decreases by $\sim 15 \%$ from 10 to $85{ }^{\circ} \mathrm{C}$. As expected, the excitation spectra are very similar to the absorption spectra, and there is very little change in the excitation spectra as the temperature is increased from 10 to $85^{\circ} \mathrm{C}$ as shown in Figure 5 (left). The luminescence spectra change with temperature. The right part of Figure 5 shows that as the temperature is raised the band width of the emission increases, and the peak position shows a slight red-shift.
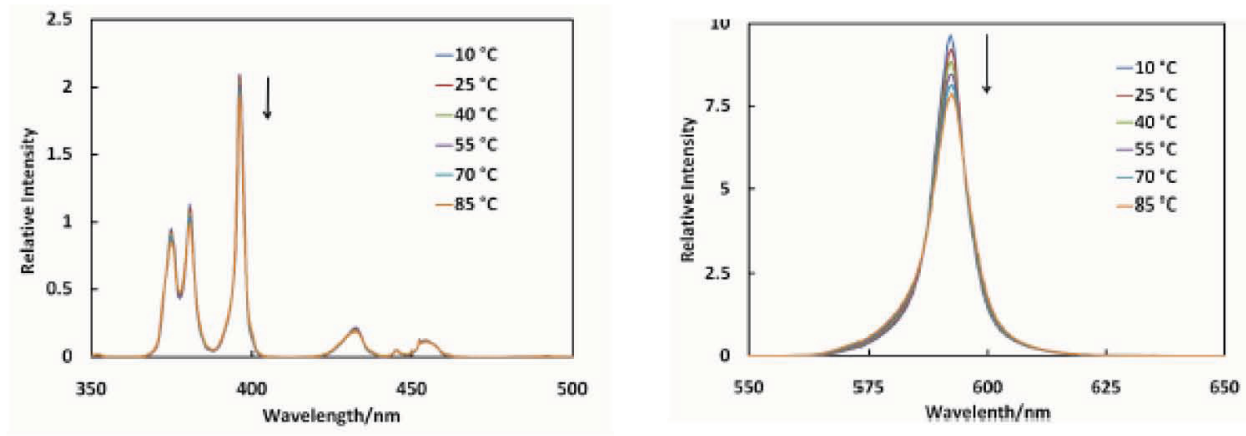

Figure 5. Excitation (left) and emission (right) spectra of $\mathrm{Cm}$ (III) in $0.001 \mathrm{M} \mathrm{HClO}_{4}$ in $\mathrm{H}_{2} \mathrm{O}$ at different temperatures.

\subsubsection{Quantum yield, radiative rate constant and the correlation for calculating the hydration number}

In this work, the quantum yield, $\Phi_{C m(I I I)}$, was measured in pure $\mathrm{H}_{2} \mathrm{O}$ and $\mathrm{D}_{2} \mathrm{O}$ systems from 10 to $85{ }^{\circ} \mathrm{C}$. The experimentally determined $\Phi_{\mathrm{Cm}(\mathrm{III})}$ and corresponding radiative rate constant, $k_{r}$, are given in Table 3 . The data show that the $\Phi_{\mathrm{Cm} \text { (III) }}$ differ by more than one order of magnitude between the pure $\mathrm{H}_{2} \mathrm{O}$ and $\mathrm{D}_{2} \mathrm{O}$ systems but that there is only a small temperature effect for $\Phi_{\mathrm{Cm}(\mathrm{III})}$ in either the $\mathrm{H}_{2} \mathrm{O}$ or the $\mathrm{D}_{2} \mathrm{O}$ systems. However, little temperature or isotope effect was observed for the radiative decay rate constant, $k$. Therefore, we have used the average values of $0.459 \mathrm{~ms}-1$ for $k r$, and $2.18 \mathrm{~ms}$ for $\tau_{r}$ for the temperature range of 10 to $85^{\circ} \mathrm{C}$ in this work. 
Table 3 Measured quantum yields and radiative rate constants of $\mathrm{Cm}(\mathrm{III})$ in $\mathrm{H}_{2} \mathrm{O}$ and $\mathrm{D}_{2} \mathrm{O}$ as a function of temperature. *The measured values are estimated to have an accuracy of $\pm 10 \%$ in $\mathrm{H}_{2} \mathrm{O}$, and $5 \%$ in $\mathrm{D}_{2} \mathrm{O}$.

\begin{tabular}{ccccc}
\hline $\mathrm{t} /{ }^{\circ} \mathrm{C}$ & $\Phi_{\mathrm{Cm}(\mathrm{II})}{ }^{*}\left(\mathrm{H}_{2} \mathrm{O}\right)$ & $k_{r}\left(\mathrm{H}_{2} \mathrm{O}\right) \mathrm{ms}^{-1}$ & $\Phi_{\mathrm{Cm}(\mathrm{III})}{ }^{*}\left(\mathrm{D}_{2} \mathrm{O}\right)$ & $k_{r}\left(\mathrm{D}_{2} \mathrm{O}\right) \mathrm{ms}^{-1}$ \\
\hline 10 & 0.0323 & 0.466 & 0.621 & 0.467 \\
25 & 0.0296 & 0.434 & 0.620 & 0.470 \\
40 & 0.0319 & 0.473 & 0.615 & 0.469 \\
55 & 0.0288 & 0.436 & 0.605 & 0.464 \\
70 & 0.0296 & 0.455 & 0.594 & 0.459 \\
85 & 0.0289 & 0.452 & 0.590 & 0.457 \\
Average & & 0.453 & & 0.464 \\
\hline
\end{tabular}

\begin{tabular}{|c|c|c|c|}
\hline \multicolumn{4}{|c|}{$\begin{array}{l}\text { Table } 4 \text { Values of the rate constant per } \mathrm{H}_{2} \mathrm{O} \text { molecule, the ratio of the radiative rate constant } \\
\text { to the rate constant per } \mathrm{H}_{2} \mathrm{O} \text { molecule, and the correlation between } k_{0 b 5} \text { and } \eta_{\omega_{2}} \text { of } \mathrm{Cm}(\mathrm{II}) \\
\text { at different temperatures }\end{array}$} \\
\hline $\mathrm{t}^{\prime /} \mathrm{C}$ & $\left.\left(1 / k_{\mathbb{H}_{2}}^{t}\right)\right) m s$ & $k_{r} / k_{H_{R} Q}^{\prime} Q$ & Correlation between $k_{o b s}$ and $\eta_{H_{g} Q}$ of $\mathrm{Cm}$ (III) \\
\hline 10 & 0.64 & 0.30 & $N_{H_{H_{z}}}=0.64 \times k_{o b s}-0.30$ \\
\hline 25 & 0.63 & 0.29 & $W_{N_{Q} Q}=0.63 \times k_{o b s}-0.29$ \\
\hline 40 & 0.62 & 0.29 & $n_{H_{z}}=0.62 \times k_{o b s}-0.29$ \\
\hline 55 & 0.61 & 0.28 & $n_{H_{g} 0}=0.61 \times k_{o b s}-0.28$ \\
\hline 70 & 0.60 & 0.28 & $N_{N_{W_{z}} Q}=0.60 \times k_{o b s}-0.28$ \\
\hline 85 & 0.59 & 0.27 & $n_{H_{H_{L}} 0}=0.59 \times k_{a b s}-0.27$ \\
\hline
\end{tabular}

The correlations between $k_{o b s}$ and of $\mathrm{Cm}$ (III) in Table 4 are derived by correcting $k_{o b s}$ with the radiative rate constant that was derived from $\Phi$, the experimentally measured quantum yield. On the contrary, the empirical correlations previously reported in the literature are obtained by fitting $k_{o b s}$ as a function of for a number of Cm(III)-spiked lanthanide solids with known, without the correction for the radiative rate constant [9].. The empirical correlations at $25 \mathrm{oC}$ in the literature $\left(=0.65 \times k_{o b s}-0.88\right)$ is in fairly good agreement with the correlation derived from this work $\left(=0.63 \times k_{o b s}-0.29\right)$, because $k_{r}(\sim 0.45 \mathrm{~ms}-1)$ is much smaller than $k_{o b s}(\sim 15 \mathrm{~ms}-1)$ for $\mathrm{Cm}(\mathrm{III})$ in pure $\mathrm{H}_{2} \mathrm{O}$ solutions.

\subsubsection{Mechanism of temperature dependency of non-radiative decay}

The fluorescent lifetime of $\mathrm{Cm}$ (III) was found to be only weakly temperature dependent in the limited temperature range of our measurements $\left(10\right.$ to $\left.85^{\circ} \mathrm{C}\right)$. Following the equation

$$
k_{o b s}=k_{r}+A \exp (-\Delta E / R T)
$$

the plots of $\ln \left(k_{\text {obs }}-k_{\mathrm{r}}\right)$ against $1 / T$ are drawn for pure $\mathrm{H}_{2} \mathrm{O}$ and $\mathrm{D}_{2} \mathrm{O}$ systems in Figure 6 . Two linear lines with small and nearly identical slopes were obtained $\left(A=\mathrm{e}_{9.92}, \Delta E / R=102 \mathrm{~K}\right.$ in $\mathrm{H}_{2} \mathrm{O}$, and $A=\mathrm{e}_{6.06}, \Delta E / R$ $=106 \mathrm{~K}$ in $\mathrm{D}_{2} \mathrm{O}$ ), suggesting that the de-excitation mechanisms for the ${ }_{6} \mathrm{D}^{\prime}{ }_{7 / 2}$ emission of the $\mathrm{Cm}(\mathrm{III})$ aqua ion in $\mathrm{H}_{2} \mathrm{O}$ and $\mathrm{D}_{2} \mathrm{O}$ have the same weak temperature dependence. The observed activation energy is 
almost the same in $\mathrm{H}_{2} \mathrm{O}$ and $\mathrm{D}_{2} \mathrm{O}$, about $0.9 \mathrm{~kJ}$ mol-1, which is very close to the energy gap between the first and second ( $\mathrm{A}_{1}$ and $\mathrm{A}_{2}$ ) levels of the metastable ${ }_{6} \mathrm{D}^{\prime}{ }_{7 / 2}$ multiplets for $\mathrm{Cm}$ (III) in aqueous solution and in a hydrated triflate crystal, as reported by Lindqvist, et al. [10]. Hence, the weak temperaturedependence of the luminescence quenching of $\mathrm{Cm}$ (III) aqua ion in $\mathrm{H}_{2} \mathrm{O}$ and $\mathrm{D}_{2} \mathrm{O}$ can be ascribed to the thermal population within the ${ }_{6} \mathrm{D}_{7 / 2}$ multiplet. In this case, as the temperature is increased, the thermal population of the $\mathrm{A}_{1}$ level is reduced and the thermal population of the $\mathrm{A}_{2}$ level (and higher levels) are increased resulting in a shortened observed lifetime of the $A_{1}$ emitting level.

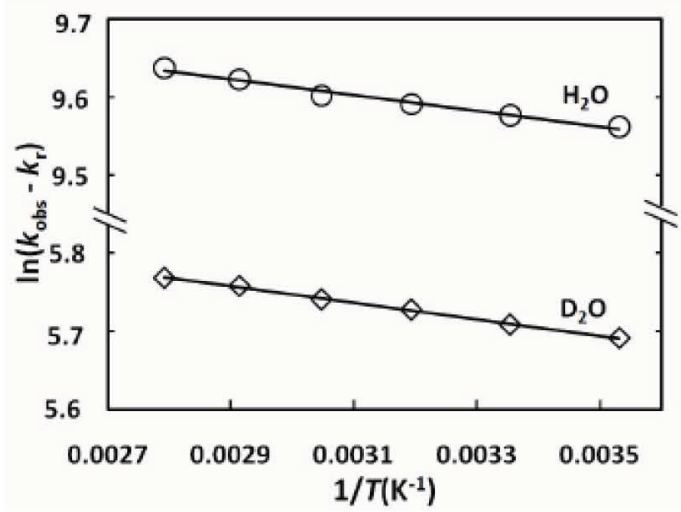

Figure 6 Thermal quenching of $\mathrm{Cm}$ (III) in pure $\mathrm{H}_{2} \mathrm{O}$ and $\mathrm{D}_{2} \mathrm{O}$ (with $k_{\text {ots }}$ and $k_{r}$ in units of $s^{-1}$ )

In conclusion, by measuring the quantum yield and the luminescence life time, we have established the correlation between the hydration number and luminescence lifetime in the temperature range from 10 to $85^{\circ} \mathrm{C}$. These correlations will be used to calculate the hydration numbers for $\mathrm{Cm}$ (III) aqua species at different temperatures in the studies of the complexation of $\mathrm{Cm}$ (III) with TALSPEAK ligands.

\section{PUBLICATIONS}

- G. Tian, L. R. Martin, Z. Zhang, L. Rao, Thermodynamic, Spectroscopic and Computational Studies of Lanthanide Complexation with Diethylenetriaminepentaacetic acid: Temperature Effect and Coordination Modes, Inorg. Chem., 2011, 50 (7), 3087-3096.

- G. Tian, N. M. Edelstein, L. Rao, Spectroscopic Properties and Hydration of the Cm(III) Aqua Ion from 10 to 85 .C, J. Phys. Chem. A, 2011, 115 (10), 1933-1938.

- L. Rao, G. Tian, Effect of Temperature on the Complexation of Cm(III) with Nitrate in Aqueous Solutions, Dalton Trans. 2011, 40 (4), 914 - 918.

- G. Tian, L. R. Martin, L. Rao, Complexation of Lactate with Nd(III) and Eu(III) at Variable

Temperatures: Studies by Potentiometry, Microcalorimetry, Optical Absorption and Luminescence Spectroscopy, Inorg Chem. 49, 10598-10605 (2010).

- G. Tian, L. Rao, Effect of Temperature on the Protonation of the TALSPEAK Ligands: Lactic and Diethylenetrinitropentaacetic Acids, Separation Science and Technology, 45, 1718-1724 (2010).

\section{FUTURE PLAN}

\subsection{Complexation of $\mathrm{Cm}(\mathrm{III})$ with lactate and DTPA at variable temperatures}

Now that we have obtained a better understanding of the hydration state of $\mathrm{Cm}$ (III) in aqueous solutions and measured the complexation of Cm(III) with nitrate (a "background" ligand in TALSPEAK) at 10 to $85 . \mathrm{C}$, we are ready to investigate the complexation of $\mathrm{Cm}(\mathrm{III})$ with lactic acid and DTPA, in comparison with the previous studies of $\mathrm{Nd}(\mathrm{III})$ and $\mathrm{Eu}(\mathrm{III})$. Luminescence spectroscopy will still be the major 
technique for these studies. The thermodynamic data on $\mathrm{Cm}$ (III), together with those on the lanthanides, will provide more accurate prediction of the behavior of actinides/lanthanides in TALSPEAK as the operation temperature changes.

\subsection{Extension of the thermodynamic and speciation studies from aqueous phase to organic phase, and to the two-phase (org/aq) system}

On the basis of the studies of the interactions between $\mathrm{Ln}(\mathrm{III}) / \mathrm{Cm}(\mathrm{III})$ and the TALSPEAK ligands (nitrate, lactate and DTPA) in the aqueous phase, the chemical speciation of $\operatorname{Ln}(\mathrm{III}) / \mathrm{Cm}$ (III) in the aqueous phase and at variable temperatures is clearly understood. We will extend the thermodynamic and speciation studies of $\mathrm{Ln}(\mathrm{III}) / \mathrm{Cm}$ (III) under TALSPEAK process from the aqueous phase to the organic phase and the org/aq two phase systems. Optical absorption and luminescence spectroscopic techniques will be used. Integration of the data for the aqueous and organic phases will allow us to propose the most likely mechanism of the extraction.

\section{REFERENCES}

1. Horrocks Jr., W. DeW.; Sudnick, D. R. J. Am. Chem. Soc. 1979, 101, 334.

2. Barthelemy, P. P.; Choppin, G. R. Inorg. Chem. 1989, 28, 3354.

3. Kimura, T.; Choppin, G. R. J. Alloys and Compd. 1994, 213, 313.

4. Mondry, A.; Starynowicz, P. Polyhedron, 2000, 19, 771

5. Inomata, Y.; Sunakawa, T.; Howell, F. S. J. Mol. Struct. 2003, 648, 81.

6. Bryden, C. C.; Reilley, C. N. Anal. Chem. 1982, 54, 610.

7. Liu B.; Wang Y. F.; Wang J.; Gao J.; Xu R.; Kong Y. M.; Zhang L. Q.; Zhang X. D. J. Structural Chemistry 2009, 50, 880-886.

8. Harder, R.; Chaberek, S. J. Inorg. Nucl. Chem. 1959, 11, 197-209.

9. Kimura, T.; Choppin, G. R. Journal of Alloys and Compounds 1994, 213/214, 313.

10. Lindqvist-Reis, P.; Apostolidis, C.; Rebizant, J.; Morgenstern, A.; Klenze, R.; Walter, O.; Fanghanel, T.; Haire, R. G. Angewandte Chemie-International Edition 2007, 46, 919. 


\section{Appendix B}

\section{Separation Process Thermodynamics and Kinetics: Development of microfluidic devices for solvent extraction studies and radioanalytical applications}

Dr. Artem Guelis

Argonne National Laboratory 


\section{SUMMARY}

One bottleneck in the design of next-generation solvent extraction-based nuclear fuel reprocessing schemes is a lack of interfacial mass transfer rate constants obtained under well-controlled conditions for lanthanide and actinide ligand complexes - such rate constants are a prerequisite for mechanistic understanding of the extraction chemistries involved, and are of great assistance in the design of new chemistries. In addition, rate constants obtained under conditions of known interfacial area have immediate, practical utility in models required for the scaling-up of laboratory-scale demonstrations to industrial-scale solutions. Existing experimental techniques for determining these rate constants suffer from two key drawbacks: either slow mixing, or unknown interfacial area. The volume of waste produced by traditional methods is an additional, practical concern in experiments involving radioactive elements, both from disposal cost and experimenter safety standpoints. In this project, we test a plugbased microfluidic system that uses flowing plugs (droplets) in microfluidic channels to determine absolute interfacial mass transfer rate constants under conditions of both rapid mixing and controlled interfacial area. We utilize this system to determine, for the first time, the rate constants for interfacial transfer of all lanthanides, minus promethium, plus yttrium, under TALSPEAK process conditions, as a first step towards testing the molecular mechanism of this separation process.

\section{Introduction and Significance}

Solvent extraction kinetics experiments have three primary uses of interest to the FCR\&D program: 1) providing insight into the mechanism of complex solvent extraction chemistries; 2) establishing reaction rates for kinetically limited extraction chemistries for use in process modeling and design; and 3) characterizing the efficiency of solvent extraction process equipment.

First, the mechanism of complex solvent extraction chemistries can be determined through sufficiently detailed, well-controlled kinetics experiments. By varying the relevant experimental parameters $(\mathrm{pH}$, extractant concentration, etc.) the role of each individual reaction component can be deduced. While Dvalues alone are sufficient to gain such insight for many experimental systems, rate constants provide more useful information, particularly for complex chemical systems.

Second, modeling of non-equilibrium solvent extraction processes requires mass-transfer coefficient data. Process modeling for solvent extraction chemistries attempts to predict the output of each contactor stage based on the system's inputs and process conditions. For kinetically limited (slow) solvent extraction chemistries, processes cannot be assumed to be at equilibrium in each stage. To realistically model and design such processes, experiments are required to measure the mass transfer coefficients for the system. Precise values would permit accurate modeling, which could allow the eventual scale-up of chemistries with desirable D-values but slow extraction, such as the TALSPEAK process.

Third, solvent extraction kinetics can be used to characterize process equipment itself, even when operated in steady-state conditions. The "efficiency" term commonly used to describe process equipment can be inferred experimentally by calculating the apparent average specific interfacial area of a process tool by running a kinetically well-defined process at a known residence time.

\section{Comparison of Kinetics Experimental Methods}

Currently, there are no satisfactory methods to quantify, with known interfacial areas, the rate constants of interfacial mass transfer (i.e. $k_{a o}$ and $k_{o a}$ in Figure 1A) for radioactive species that move slowly across interfaces during solvent extraction. Existing systems suffer from a combination of practical limitations, and fundamental deficiencies. Two types of systems are routinely used to measure the kinetics of solvent 
extraction and interfacial mass transfer, but do not necessarily provide data that can provide mechanistic insight, or translate to industrial systems. In addition, both methods use bulk quantities of fluids and therefore can be difficult to use safely with highly radioactive materials, which may require high dilutions, shielding, or even remote operation, and are expensive to dispose of as experimental waste. ${ }^{14}$ The first system is a highly stirred tank or a vortex mixing system (Figure 1Bi), and is typically analyzed by gamma counting or ICP-MS to determine the metal content of aliquots of each phase. Mixing is rapid, so relative reaction rates can be identified. However, the data are device-specific, interfacial area is unknown and unpredictable, and removing aliquots for analysis affects the specific interfacial area. These tanks also generate large volumes of waste $(10-100 \mathrm{~mL}$ volumes), so both safety and cost of disposal of experimental waste can be problematic. Because the interfacial area is unknown, it is typically not feasible to carefully vary the experimental conditions to determine the specific reaction mechanism.

A) TALSPEAK metal solvent extraction

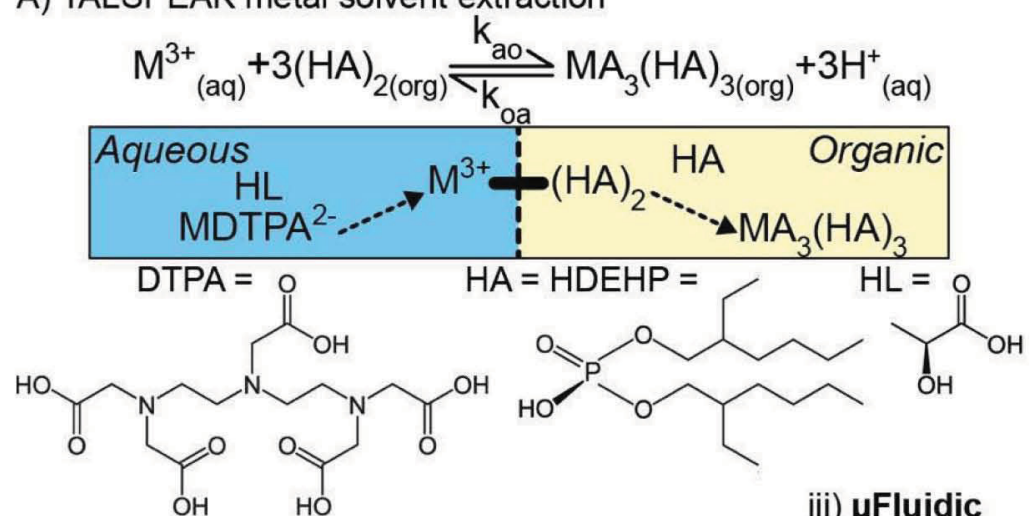

B) i) Stirred Tank

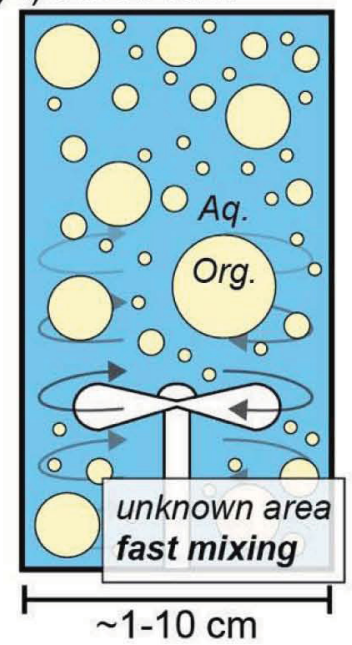

ii) Lewis Cell

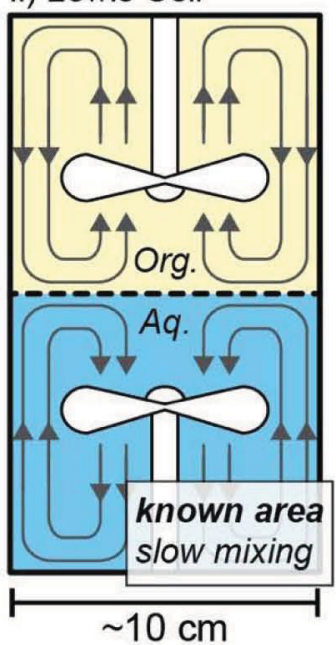

iii) $\boldsymbol{\mu}$ Fluidic

(this work)

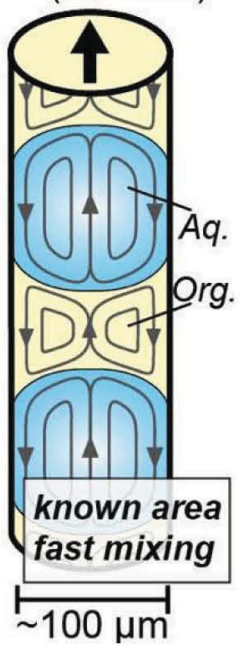

Figure 1. A) Chemistry of metal-ion separation via solvent extraction under TALSPEAK conditions. The TALSPEAK process is one step in a proposed recycling scheme of nuclear fuel, which separates lanthanides $\left(\mathrm{M}^{3+}\right)$ from actinides by extracting the lanthanides from an aqueous phase (blue) to an organic phase (yellow). B) Schematic illustration comparing the traditional methods for measuring kinetics (i,ii) with the method tested here (iii). (i) Highly-stirred tanks provide turbulent convective mixing and rapid mixing, but the specific interfacial area is unknown and uncontrolled. (ii) A Lewis cell provides known specific interfacial area (1-100 $\left.\mathrm{m}^{-1}\right)$ with slow diffusive mixing. (iii) Droplet-based microfluidic devices provide rapid mixing, known and large interfacial area $\left(10000 \mathrm{~m}^{-1}\right)$, and absolute quantification with high time resolution. 


\section{Highly stirred tank}

The first system is a highly stirred tank or a vortex mixing system (Figure 1Bi), and is typically analyzed by gamma counting or ICP-MS to determine the metal content of aliquots of each phase. Mixing is rapid, so relative reaction rates can be identified. However, the data are device-specific, interfacial area is unknown and unpredictable, and removing aliquots for analysis affects the specific interfacial area. These tanks also generate large volumes of waste $(10-100 \mathrm{~mL}$ volumes), so both safety and cost of disposal of experimental waste can be problematic. Because the interfacial area is unknown, it is typically not feasible to carefully vary the experimental conditions to determine the specific reaction mechanism.

\section{Lewis cell}

The second system commonly used to measure the kinetics of solvent extraction is a Lewis cell (Figure 1Bii), a slow, laminar mixer operated at relatively low Reynolds numbers to maintain a quiescent interface. Lewis cells are typically analyzed by continuous optical or radiometric monitoring, and have a known and constant interfacial area. However, the specific interfacial areas that are feasible are small (1 $100 \mathrm{~m}^{2} / \mathrm{m}^{3}$ ) and result in slow mixing and slow rates of reaction. Processes with small rate constants, such as TALSPEAK, can take days to reach equilibrium in a Lewis cell, but this is the same timescale as the half-lives of some of the radionuclide tracers of interest. Again, safety concerns and disposal costs can be prohibitively high. In addition, only a few cations can be distinguished by continuous monitoring, only a few lanthanides have characteristic bands in the UV-VIS to near-IR range, making Lewis cells impractical for real-time monitoring of realistic mixtures of cations. These systems have a non-negligible diffusion zone, where no active mixing takes place between the two phases. This diffusion zone is typically estimated to be between approximately $50 \mu \mathrm{m}$ and $100 \mu \mathrm{m} . \frac{15}{}$ It can take $2-20$ seconds for a metal cation to cross via diffusion, and significantly longer for ions complexed with extractants. Thus the main problem of slow mixing in this system is that interfacial transfer becomes limited not by intrinsic kinetics, but rather by diffusion of molecules to the interface, and therefore it becomes impossible to obtain correct rate constants for reactions with rapid transfer kinetics.

\section{Droplet-based Microfluidics}

Droplet-based microfluidic techniques are well-suited for obtaining absolute rate constants for interfacial mass transfer, due to their unique ability to maintain a constant interfacial area while simultaneously achieving rapid mixing. Droplet microfluidics is well-established ${ }^{17}$ as a method to quantify chemical kinetics using microliter volumes of sample, and has several advantages that make it suitable for the TALSPEAK system. Both fast (millisecond) and slow (hours to days) reactions have been measured, $\frac{18-22}{}$ and rapid mixing eliminates the effects of diffusion as a confounding factor. ${ }^{23}$ Because the interfacial area is measured directly from the droplet size, the results are generalizable, and additional mechanistic insights can be obtained. Additionally, each nL-volume droplet can be considered to be a separate experimental trial, which allows for hundreds or thousands of trials to be performed with just a few $\mu \mathrm{L}$ of sample. While many droplet-based microfluidic devices have been made in soft elastomers such as PDMS, other materials have also been used to handle harsh chemical systems. ${ }^{24}, 25$ Microfluidic methods have also been developed both to separate an aqueous phase from an organic phase in a short (subsecond) timeframe $e^{26}$ and to perform the reverse process of phase injection. ${ }^{27}$ Rapid, complete isolation of the aqueous phase at a known time point provides high temporal resolution. Microfluidic systems are especially suitable for solvent extraction studies with used nuclear fuel because the small volumes required minimize exposure of lab personnel to radioactive solutions and reduce the high costs of handling and disposing of radioactive waste. Microfluidic systems have also been demonstrated for micro-scale solvent extraction intended for sample pre-treatment or small-scale production purposes. $, \underline{26}, \underline{28-}$ $\underline{32}$

In this project, we tested a plug-based microfluidic system that relies on aqueous droplets surrounded by an organic phase to measure absolute rate constants for interfacial mass transfer under TALSPEAK 
conditions for each metal ion in a model mixture of used nuclear fuel. The device has the mixing speed, large specific interfacial area, and temporal resolution of highly stirred tanks, the control of a Lewis cell, and the rapid and complete phase separation that is unique to microfluidics. We first tested the device by comparing to previously published data obtained in Lewis cells. We further used it to determine, for the first time, the rate constants for 15 elements under TALSPEAK conditions (all lanthanides, minus promethium, plus yttrium) in a first step towards testing the molecular mechanism of the separation process.

\section{Approach}

\section{Device Design and Characterization}

In order to measure the rate constant for interfacial mass transfer in the TALSPEAK system, the device must have three key properties: 1) constant interfacial area (uniform droplet generation); 2) rapid mixing to operate in the "kinetic" regime; and 3) rapid, high purity phase separation. To generate the droplets, we used a flow-focusing geometry $\frac{33-35}{3}$ in laser micromachined tubing with two concentric pieces of FEP tubing (Figure 2i). To separate the aqueous phase from the organic phase at the end of the reactor, we used laser micromachining to create holes in the inner tubing (see Figure S1, Supporting Information). These holes had precise dimensions that were calculated to exclude the aqueous phase and completely separate the organic phase at the applied pressures (Figure 2ii). Details of device fabrication are provided in the Supporting Information (see Experimental Section and Figure S2). A syringe pump controlled the flow rates of organic and aqueous streams into the outer and inner tubing respectively. The organic-toaqueous ratio can be controlled by varying syringe sizes, or by using two independent syringe pumps. The flow rate determined the residence time in the system, and varying flow rates allowed multiple contact times to be probed. A second syringe pump was used to set the flow rate of withdrawal from the phase separator; the withdrawn fluid was mostly or purely organic solution. Pure aqueous ( $>99.99 \%$ aqueous) outflow was collected in aliquots from an open outlet for offline analysis. Phase separation was rapid, taking place over less than $300 \mu \mathrm{m}$ of channel length. Each time point for an experiment that characterized the kinetics of extraction of $1 \mathrm{mM}$ total metal ions (all lanthanides minus promethium, plus yttrium, ) in $1 \mathrm{M}$ ammonium citrate, and $0.05 \mathrm{M}$ DTPA, $\mathrm{pH}=3.55$, with an organic phase of $1 \mathrm{M}$ HDEHP in n-dodecane required $10 \mu \mathrm{L}$ of aqueous phase for ICP-MS analysis, and produced a total of $<30 \mu \mathrm{L}$ of liquid waste (total of aqueous and oil).

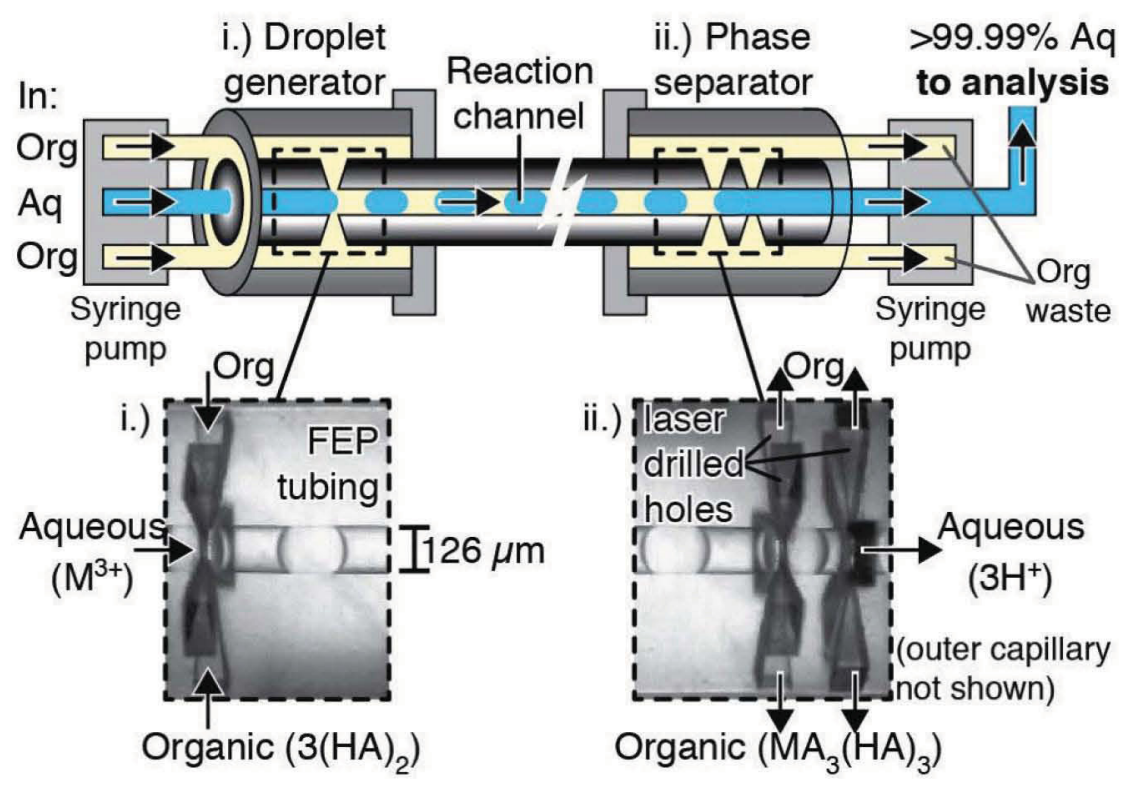


Figure 2. Device design and operation to measure rate constants of interfacial mass transfer in solvent extraction. (top) A schematic shows the design of the device. A single piece of Teflon ${ }^{\circledR}$ FEP tubing was micromachined to contain a droplet generator (i), a channel where the extraction occurs (reaction channel), and a phase separator (ii). One syringe pump (left, gray box) is used to control the inflow rates, and a second syringe pump (right, gray box) is used to set the rate of outflow of separated organic phase. The aqueous outflow is collected in aliquots for offline analysis. Additional Swagelok hardware (not shown above but detailed in SI) is utilized to properly seal the multiple pieces of tubing, and direct the output capillaries from the syringe pump (bottom) Photos of the regions in the schematic outlined by black dashes show the micromachined holes in the inner FEP tubing: one set of five holes for the droplet generator (i) and two parallel sets of five holes for the phase separator (ii).

\section{Uniform Droplet Generation}

Previous work in droplet microfluidics demonstrated that droplets can be generated with regular volumes and that the volumes can be controlled for a wide range of biologically relevant aqueous solutions. $0,36,37$ When forming droplets at a $\mathrm{T}$-junction, the volume of the droplet is a function of the capillary number, $\mathrm{Ca}$, with three distinct droplet-volume regimes. Therefore, for a specific interfacial area, $A / V$ (surface area/volume, $\mathrm{mm}^{2} / \mathrm{mm}^{3}$ ), as a function of capillary number, there should be two regimes with constant interfacial area and one regime with a continuously variable interfacial area $\frac{20,37}{3}$ In this project, only the constant interfacial area regimes were utilized. However, the variable interfacial area regime could in principle also be utilized, with additional experimental controls.

Solutions used in solvent extraction of lanthanides and actinides contain a variety of surface-active agents such as HDEHP, which could affect the dynamics of droplet formation. To verify that these surface-active agents would not negatively affect droplet formation, we first measured the specific interfacial area of droplets composed of these solutions formed in this flow-focusing device over the full range of flow rates required for kinetics experiments (Figure 3). The flow-focusing junction was a series of five $1 \mu \mathrm{m} \times 10 \mu \mathrm{m}$ slits laser machined around the circumference of a $63 \mu \mathrm{m}$-radius Teflon ${ }^{\circledR}$ FEP tube. Droplets were generated at the indicated flow rates and visually inspected under a microscope to ensure that jetting was not occurring. Then the flow was stopped, and droplets were photographed to measure volume. The dependence of interfacial surface area on flow rate was sigmoidal, with the inflection point occurring at $\mathrm{Ca} \sim 0.5$, in agreement with previously published results for droplet formation in flow-focusing geometries. ${ }^{38}$ For a given flow rate, the droplet volume and therefore the specific interfacial was very regular (Figure 3 inset), with $<7 \%$ coefficient of variation. All further experiments were carried out in the regime of constant low specific interfacial area, i.e. at low flow rates $(\leq 33 \mu \mathrm{L} / \mathrm{min})$. 


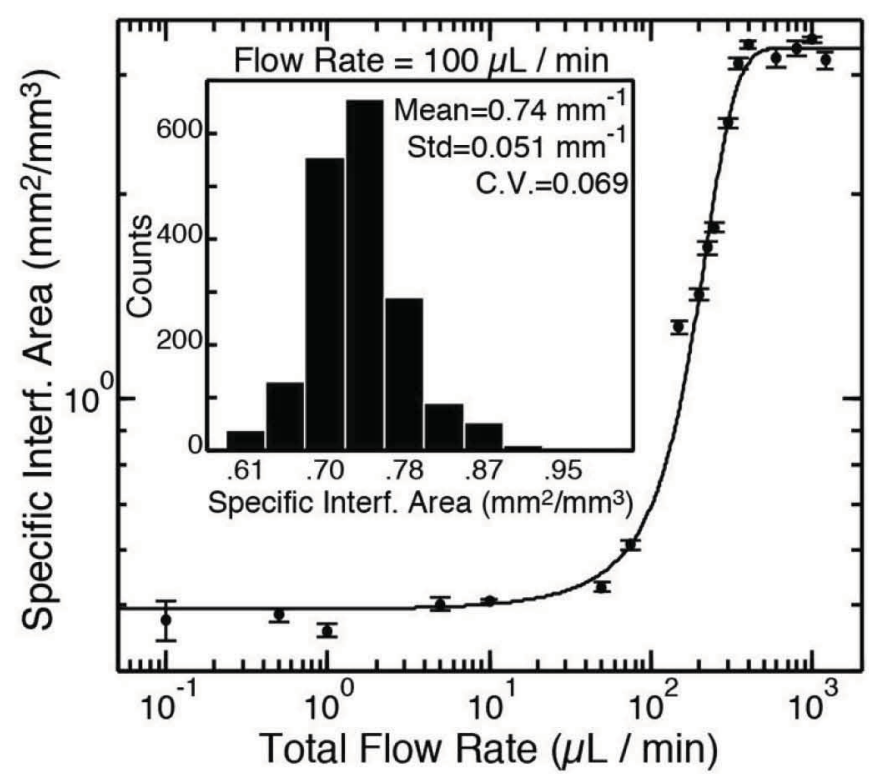

Figure 3. The specific interfacial area of droplets formed in the device is constant for a wide range of flow rates/time points. The graph shows the specific interfacial areas obtained at flow rates for aqueous droplets consisting of $1 \mathrm{mM}$ total metal ions (all lanthanides minus promethium, plus yttrium,), $1 \mathrm{M}$ ammonium citrate, and $0.05 \mathrm{M}$ DTPA, $\mathrm{pH}=3.55$, in an organic phase of $1 \mathrm{M}$ HDEHP in n-dodecane. The organic to aqueous ratio was $1: 2$. The ambient temperature was $20.0^{\circ} \mathrm{C} \pm 0.5^{\circ} \mathrm{C}$. The error bars indicate two standard deviations from the mean $(\mathrm{N}=15)$. The fit line is shown to guide the eye. The $\mathrm{x}$-axis shows total inflow rate $(\mathrm{aq}+\mathrm{org}$ ). In the inset bar graph, a computer vision system was used to individually measure the size of 1,800 droplets under identical conditions, to determine the specific interfacial with a greater confidence than can be obtained with the $\mathrm{N}=15$ measurements. The computer vision system was not utilized at flow rates greater than $100 \mu \mathrm{L} /$ minute due to frame rate limitations.

\section{Rapid Mixing: Operation in the Kinetic Regime}

Analysis of interfacial mass transfer rate constants is greatly simplified when convective mixing is much faster than diffusion to the interface or the rate of interfacial mass transfer. In this case, the rate-limiting process is mass transport across the interface and the system is in the "kinetic" regime as opposed to the "diffusion-limited" regime, $\frac{14}{}$ enabling measurements of chemically meaningful rate constants. In a Lewis cell (Figure 1Bii), the standard experiment to demonstrate operation in the kinetic regime is to increase the speed of the propeller and measure the rate constant as a function of propeller (mixing) speed. As the mixing speed increases, the rate constant reaches an asymptote where mixing is considered sufficiently faster than diffusion or the reaction of interest.

To achieve rapid mixing in the plug-based microfluidic device, we chose the flow rate, size of the channels, and size of droplets such that convection strongly accelerates diffusive mixing. Mixing is rapid in plug-based microfluidics: the small dimensions result in short diffusion lengths, and the shear forces at the channel walls induce rapid convective recirculation within each droplet and segment of carrier fluid. 41 While the microfluidics community sometimes uses the words "plugs" and "droplets" interchangeably, we emphasize that rapid mixing requires plugs - droplets that are pushing against walls of the channel to a sufficient extent to cause recirculation inside the droplet phase $e^{42,43}$ - and we used plugs for all experiments reported here. For a given channel geometry and molecular diffusion coefficient, the mixing speed is a nonlinear function of flow rate and plug length ${ }^{20,39-41}$ Millisecond-scale mixing is readily achieved in such systems as long as the cross-sectional dimensions are small, and can be accelerated by introducing chaotic flows. $\frac{18,19}{19}$ To facilitate rapid mixing, we used tubing with an inner diameter of $\sim 125$ 
$\mu \mathrm{m}$ and short plug lengths (length/width ratio $\sim 1.5-2$ ). In this device, the analytically predicted mixing time $^{41}\left(\mathrm{t}_{\text {mix }}\right)$ ranged from an absolute high of 0.5 seconds to a low of 0.012 seconds for flow rates ranging from 1 to $100 \mu \mathrm{L} / \mathrm{min}$ (equations used to generate predictions are detailed in the Supporting Information). As described below, the fastest reactions we measured were $\sim 5 \mathrm{~s}$ (half time of reaction) for $\mathrm{La}^{3+}$, from an aqueous phase with $0.05 \mathrm{M}$ DTPA and $1 \mathrm{M}$ ammonium citrate $(\mathrm{pH}=3.55)$ into an organic phase of $1 \mathrm{M}$ HDEHP in n-dodecane at an organic to aqueous ratio of $1: 2$ and an ambient temperature of $20.0^{\circ} \mathrm{C} \pm 0.5$ ${ }^{\circ} \mathrm{C}$. Such rapid experiments are performed with shorter residence times at higher flow rates, and therefore with faster mixing. This indicates that mixing should not be rate-limiting in our system. To test this prediction experimentally, we compared rate constants $\left(k_{a o}\right)$ obtained for extraction of $\mathrm{La}^{3+}$ as a function of mixing time. The details of these experimental measurements are described in subsequent sections. We varied mixing time experimentally by varying the flow rate, while varying the length of the reaction channel proportionally to keep the time of reaction constant. As shown in Table 1, we found that the rate constant for interfacial mass transfer, $k_{a o}$, was the same for all mixing times tested, indicating that our device is operating in the kinetic regime. Thus, the kinetics of interfacial mass transport reactions must indeed be rate-limiting.

\section{Rapid, Pure Phase Separation}

The kinetics of extraction of rare earth metals are typically determined by measuring the aqueous metal concentration over time using highly-accurate off-line analysis methods such as ICP-MS. This method requires isolation of the aqueous phase from the organic phase at a known time point, to prevent further extraction and to obtain an accurate measurement of lanthanide concentration. Recent microfluidic devices have shown excellent phase separation capabilities using long, extended membrane regions, ${ }^{26}$ gravity, ${ }^{28}$ a "guide structure", separation with optimal temporal resolution, here we used an analytical approach that calculated the flow rates and capillary forces necessary to separate the aqueous and organic phases with absolute purity in the shortest period of time (Figure 4).

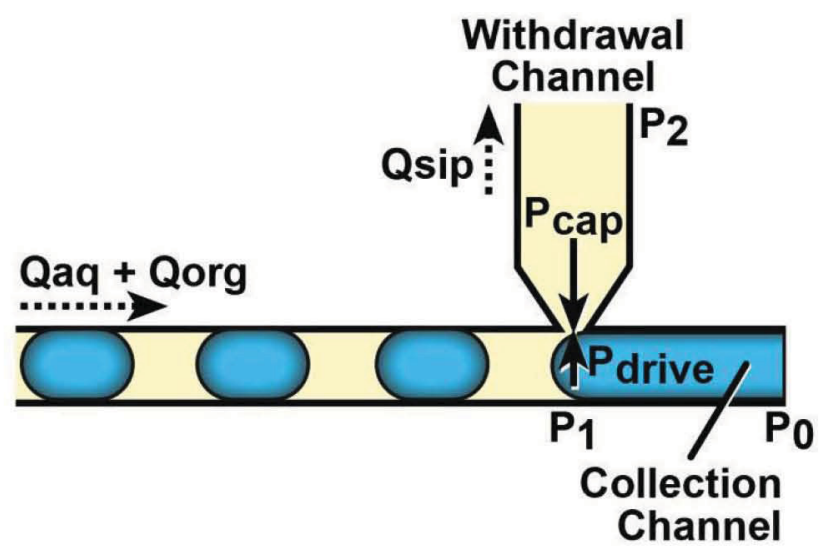

Figure 4. A schematic drawing explaining the physics of phase separation by capillary forces. Droplets of aqueous solution (blue) are moving in organic carrier fluid (yellow). Flow directions (dashed arrows) are indicated for total inflow, $Q_{a q}+Q_{o r g}$, and controlled outflow of the siphoned organic stream in the withdrawal channel, $Q_{\text {sip }}$. The aqueous sample fluid is collected from the collection channel with an open outlet. The relevant pressures are shown with solid arrows. Siphoning of aqueous solution into the withdrawal channel is regulated by the balance of the driving pressure, $\Delta P_{\text {drive }}=\left(\Delta P_{1}-\Delta P_{0}\right)-\left(\Delta P_{1}-\Delta P_{2}\right)$, against the capillary pressure at the entry to the withdrawal channel, $\Delta P_{\text {cap }}$.

The system can operate in several regimes of phase purity based on the relative rates of outflow of the siphoned stream $\left(Q_{s i p}\right)$ and inflow of the aqueous and organic phases $\left(Q_{a q}, Q_{\text {org }}\right)$ and conservation of volume. When $Q_{s i p}=0$, all fluid should bypass the withdrawal channel and be collected. In order to siphon all of the organic into the withdrawal channel, $Q_{s i p}$ must be greater than or equal to $Q_{\text {org. }}$. If $Q_{s i p}>$ 
$Q_{\text {org }}$, some aqueous solution will also be siphoned along with the organic. Perfect separation of organic and aqueous is possible in principle only if $Q_{s i p}=Q_{\text {org }}$, but this flow condition is not sufficient to provide separation. When $Q_{s i p}=Q_{\text {org }}$, the balance of capillary pressure at the entry to the withdrawal channel $\left(\Delta P_{\text {cap }},[\mathrm{Pa}]\right)$ and the driving pressure $\left(\Delta P_{\text {drive }}=\left(\Delta P_{1}-\Delta P_{0}\right)-\left(\Delta P_{I}-\Delta P_{2}\right),[\mathrm{Pa}]\right)$ determines whether or not aqueous is siphoned along with organic.

To predict the conditions under which the aqueous is excluded from the withdrawal channel, we adapted a previously published description of capillary pressure and driving pressure in microfluidic devices. .26 .45 The aqueous droplet is prevented from entering the withdrawal channel by capillary pressure, $\Delta P_{c a p}$, at the entrance:

$\Delta P_{\text {cap }}=-2 \gamma \cos \theta\left(\frac{1}{R_{o}}\right)$

Eq. 1

where $\gamma$ is the aq-org interfacial surface tension $[\mathrm{N} / \mathrm{m}], \theta$ is the contact angle of the aqueous droplet on the surface of the device in the bulk organic phase [radians], and $R_{o}$ is the radius of curvature [m] of the opening into the withdrawal channel.

The aqueous droplet is driven into the withdrawal channel by a driving pressure, $\Delta P_{\text {drive }}$, which opposes $\Delta P_{\text {cap }}$. The driving pressure is the pressure difference between a positive pressure due to flow resistance downstream in the main channel $\left(\Delta \mathrm{P}_{1}-\Delta \mathrm{P}_{0}\right)$, and a negative pressure set by withdrawing syringe pump $\left(\Delta \mathrm{P}_{1}-\Delta \mathrm{P}_{2}\right) . \Delta P_{i}$ refers to gauge pressure (pressure above ambient atmosphere) at position $P i$ (see Figure 4 for positions). If the main tubing is open to the atmosphere, then $\Delta \mathrm{P}_{0}$ is zero, and thus for a cylindrical channel such as is used here:

$$
\left(\Delta P_{1}-\Delta P_{0}\right)=\frac{8 \mu_{a q} L_{1}\left(Q_{o r g}+Q_{a q}-Q_{s i p}\right)}{\pi R_{1}^{4}} \quad \text { Eq. } 2
$$

where $\mu_{a q}$ is the viscosity of aqueous phase [Pa.s], and $L_{I}$ and $R_{I}$ are the length [m] and radius [m] of the main tubing downstream from the siphon point. The withdrawing pump sets the negative pressure in the withdrawal channel by controlling the outflow rate $Q_{s i p}>0$, such that

$$
\left(\Delta P_{1}-\Delta P_{2}\right)=-\frac{8\left(f_{\text {org }} \mu_{\text {org }}+f_{\text {aq }} \mu_{a q}\right) L_{2}\left(Q_{\text {sip }}\right)}{\pi R_{2}^{4}} \text { Eq. } 3
$$

where $\mu_{\text {org }}$ is the viscosity of organic phase [Pa·s], $f_{i}$ is the fraction of siphoned fluid consisting of phase $i$, and $L_{2}$ and $R_{2}$ are the length [m] and radius [m], respectively, of the withdrawal channel. During perfect separation only the organic phase is siphoned, so $f_{o r g}=1$ and $f_{a q}=0$. The driving pressure is given by the difference between these terms:

$$
\Delta P_{\text {drive }}=\left(\Delta P_{1}-\Delta P_{0}\right)-\left(\Delta P_{1}-\Delta P_{2}\right) \quad \text { Eq. } 4
$$

In the regime $Q_{\text {sip }} \leq Q_{\text {org }}$, this analysis predicts that the aqueous stream could be excluded from the withdrawal channel if and only if $\Delta P_{\text {drive }}<\Delta P_{\text {cap }}$; otherwise, both aqueous and organic would be siphoned. The maximum size of the opening of the withdrawal channel predicted to provide perfect separation was found by equating Eq. 1 and Eq. 4 and solving for $R_{o}$ when $Q_{s i p}=Q_{o r g}$ :

$R_{o}=-2 \gamma \cos \theta /\left(\frac{8 \mu_{a q} L_{1} Q_{a q}}{\pi R_{1}^{4}}+\frac{8 \mu_{o r g} L_{2} Q_{s i p}}{\pi R_{2}^{4}}\right)$

Eq. 5

We used the dimensions of our device and the properties of the aqueous and organic solutions used for subsequent extraction experiments (See Fluid Properties and Table S1 in Supporting Information) to 
predict the maximum size of the opening that would provide perfect separation. For flow rates up to $Q_{s i p}=$ $Q_{\text {org }}=100 \mu \mathrm{L} / \mathrm{min}$, we found that the critical $R_{o}$ was $\sim 1 \mu \mathrm{m}$. We utilized this dimension as a fabrication target for laser micromachining.

We tested the accuracy of the predictions for separation of the aqueous and organic phases by quantifying the composition of the stream exiting the collection channel as a function of the inflow and outflow rates (Figure 5). The dimensions of the device are given in Table S1 (see Supporting Information). The device had 10 exit holes for phase separation fabricated by an excimer laser (as shown in Fig. 2ii), each with $\sim 1$ $\mu \mathrm{m} \times 10 \mu \mathrm{m}$ rectangular cross-sections at the tip where they contacted the lumen of the extraction channel. Phase separation was characterized using flow rates that would produce residence times between 0.5 seconds and 1 minute. In our $88 \mathrm{~mm}$-long reaction channel with an inner radius of $63 \mu \mathrm{m}$, this corresponded to flow rates between 1 and $100 \mu \mathrm{L} / \mathrm{min}$.

As expected, with an outflow rate of zero $\left(Q_{\text {sip }}=0\right)$, both the aqueous and organic phases bypassed the separation channels and exited the system (Figure 5Bi). For $Q_{s i p}>\left(Q_{a q}+Q_{o r g}\right)$, all fluid was siphoned and no flow was observed through the sample outlet. These results agree with conservation of volume and indicated that dead volumes were small or negligible in the device. Perfect separation was predicted to occur at $Q_{s i p}=Q_{\text {org }}$ (Figure 5A) when the entry to the withdrawal channel is restricted to $\mathrm{R}_{0} \sim 1 \mu \mathrm{m}$ as discussed above. Although the aqueous stream was successfully excluded from the withdrawal channel at $Q_{\text {sip }} \leq Q_{\text {org }}$, we found that at $Q_{\text {sip }}=Q_{\text {org }}$, the aqueous stream in the collection channel still contained some of the organic phase. A higher outflow rate, $Q_{\text {sip }} \geq 1.3\left(Q_{\text {org }}\right)$, was required for the collection channel to have an aqueous stream with $>99.99 \%$ purity (Figure 5A black circles, also Figure 5Biii). There are several potential factors, not included in the model, which may be responsible for imperfect agreement between theory and experiment: i) additional resistance terms, such as flow through the narrow laserdrilled inlets to the withdrawal channels; ii) additional capillary pressure produced by droplets of aqueous solution that were siphoned into the withdrawal channel; and iii) imperfect control of the dimensions of the $1 \times 10 \mu \mathrm{m}$ opening, which is at the limit of the capabilities for excimer laser machining in soft materials. Yet, the agreement was sufficiently good that we could confidently design devices and carry out kinetic measurements. Conservation of volume predicts that some aqueous solution would be siphoned into the withdrawal channel at these flow rates, so the withdrawn organic stream was not pure. Nevertheless, because the kinetics of extraction were analyzed by measuring the lanthanide concentration in the aqueous stream, as long as the capillary pressure was sufficient to provide a pure aqueous stream, kinetic data was easily obtained. 

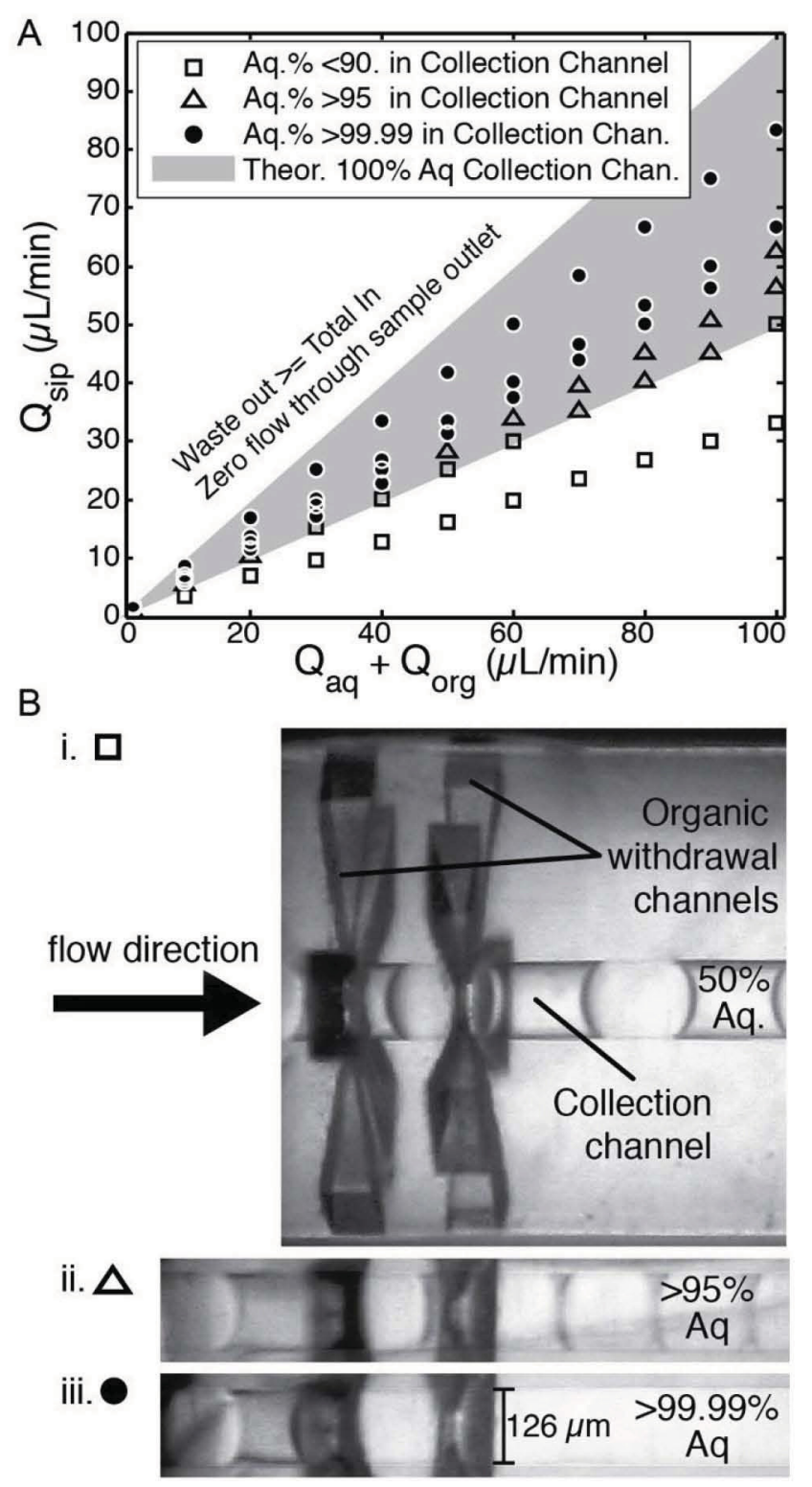

Figure 5. Characterization of purity of the collected aqueous stream after phase separation and comparison to theoretical predictions. (A) A phase diagram showing the composition of the collected sample stream (aqueous collected volume / total collected volume) as a function of inlet and withdrawal channel flow rates. The inlet rate shown corresponds to total fluid inflow ( $\left.Q_{\text {org }}+Q_{\text {aq }}\right)$. Experimental data was obtained with droplets of an aqueous solution of a mixture of 14 lanthanides and yttrium (each at $1 \mathrm{mM})$ with $1 \mathrm{M}$ ammonium citrate and $0.05 \mathrm{M}$ DTPA $(\mathrm{pH}=3.55)$ in a carrier fluid of $1 \mathrm{M}$ HDEHP in dodecane. Organic to aqueous ratio was $1: 2$. Ambient temperature was $20.0^{\circ} \mathrm{C} \pm 0.5{ }^{\circ} \mathrm{C}$. (B) Photos of the device during use at three different inlet/outlet ratios, resulting in i) zero flow through the phase separation channels, i.e. $50 \%$ aqueous in the sample outflow; ii) $>95 \%$ aqueous in the sample outflow; and iii) $>99.9 \%$ aqueous in the sample outflow. The device consisted of 10 phase separation channels; two rows of 5 radially arranged phase separation channels are visible in the photos, each with $\sim 1 \mu \mathrm{m} x$ $10 \mu \mathrm{m}$ cross-section at the narrow end. Scale is the same for all photos. 


\section{Results}

\section{Comparison with previously published results}

After verifying that the device could provide rapid, pure phase separation, we used the device to determine the concentration of lanthanide ions in the aqueous phase, $C$, vs. time during extraction (see Experimental Section in Supporting Information for additional details). All experiments, except those specifically designed to test the effects of mixing channel length, were carried out in $88 \mathrm{~mm}$-long tubing with an internal radius of $63 \mu \mathrm{m}$. In this $88 \mathrm{~mm}$-long tubing, different flow rates were utilized to set different residence times, and the final concentrations in the aqueous phase were individually plotted. Typically, 15 points were taken to cover the range from $\sim 2$ to $\sim 450 \mathrm{~s}$, with the majority of the points taken towards the beginning of the experiment. For example, Figure 6 shows the transfer of $\mathrm{Eu}^{3+}$ from an aqueous phase $(0.05 \mathrm{M}$ (2hydroxyethyl)ethylenediamine-N, N, N' -triacetic acid (HEDTA), $1 \mathrm{M} \mathrm{NaCl}$, $0.2 \mathrm{mM}$ lactic acid, $\mathrm{p}[\mathrm{H}] 3.00$ ) into an organic phase containing 0.01 M HDEHP in dodecane. The organic to aqueous ratio was $1: 1$. The ambient temperature was $20.0^{\circ} \mathrm{C} \pm 0.5^{\circ} \mathrm{C} .10 \mu \mathrm{L}$ of aqueous solution was collected for each data point. Since each aqueous droplet is approximately $2 \mathrm{~nL}$, this $10 \mu \mathrm{L}$ sample represents approximately 5,000 individual trials averaged together.

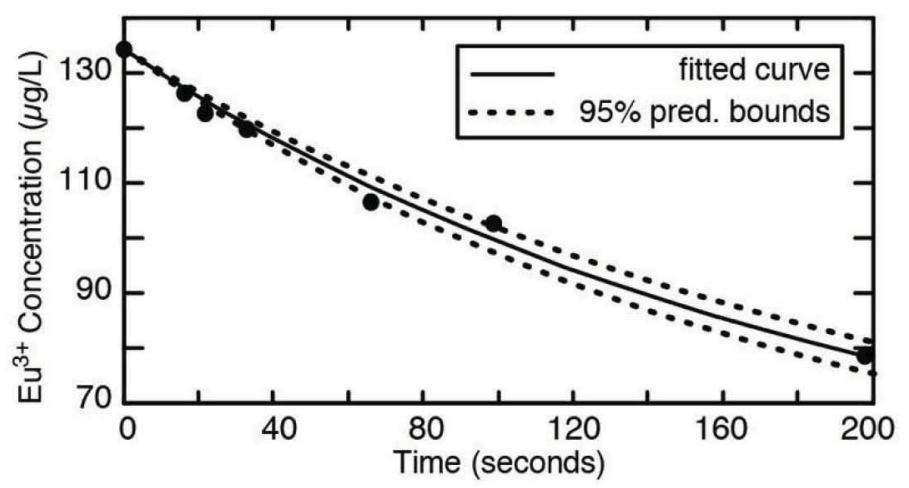

Figure 6. Primary concentration vs. time data for extraction obtained using the tested device under conditions that duplicated previously published results: $: \frac{46}{}$ the aqueous phase initially contained $\mathrm{Eu}^{3+}$ at 134 $\mu \mathrm{g} / \mathrm{L}$ and $0.05 \mathrm{M}$ HEDTA, $1 \mathrm{M} \mathrm{NaCl}, 0.2 \mathrm{mM}$ lactic acid at a $\mathrm{p}[\mathrm{H}]$ of 3.00. The organic to aqueous volumetric ratio was $1: 1$. The ambient temperature was $20.0^{\circ} \mathrm{C} \pm 0.5^{\circ} \mathrm{C}$. The decay shows the transfer into an organic phase containing $0.01 \mathrm{M}$ HDEHP in dodecane. Circles indicate primary data points obtained using the tested method and ICP-MS. The curves are fit using Eq. 7. Prediction bounds were calculated using the predint function in MATLAB 7.6 and indicate the upper and lower $95 \%$ prediction bounds for the curve fit using Eq. 7.

All data were analyzed according to a first order decay equation for the equilibrium $C \leftrightarrows C_{o r g}$, with forward and backward interfacial mass transfer rate constants $k_{a o}$ and $k_{o a}$ respectively, distribution ratio $K_{D}=k_{a d} k_{o a}=C_{o r g, e q} / C_{e q}$, and initial aqueous concentration $C_{0}$. Assuming that $C_{o r g}=0$ initially and that there are no side reactions to consume the aqueous metal ions, the rate equation is

$\mathrm{d} C / \mathrm{d} t=(A / V)\left(k_{o a} C_{o r g}-k_{a o} C\right) \quad$ Eq. 6

Integration over $t$ from 0 to $t$ and over $C$ from $C_{0}$ to $C$ gives ${ }^{47.48}$ 


$$
\frac{C_{e q}-C}{C_{e q}-C_{0}}=e^{-k_{o a}(A / V)\left(1+\left(V_{o r g} / V_{a q}\right) K_{D}\right) t}
$$

\section{Eq. 7}

Here, $V_{o r g} / V_{a q}$ is the ratio of the volumetric flowrate of the organic phase to the aqueous phase. Concentration vs. time data from experiments was fit according to Eq. 7, and 95\% prediction intervals were plotted (Figure 6). All parameters except $k_{o a}[\mathrm{~mm} / \mathrm{s}]$ are known from independent experimental measurements (see Experimental Section in Supporting Information), therefore $k_{o a}$ can be determined by fitting the data to Eq. 7. From the fitted $k_{o a}$ and known $K_{D}$, we solved for the forward interfacial mass transfer rate constant $k_{a o}[\mathrm{~mm} / \mathrm{s}]$

$k_{a o}=k_{o a} K_{D} \quad$ Eq. 8

The device-independent interfacial mass transfer rate constants are multiplied by the specific interfacial area $A / V[1 / \mathrm{mm}]$ to obtain the device-specific rate of interfacial mass transfer $[1 / \mathrm{s}]$.

First, we tested whether the device produced data that was consistent with results obtained by traditional methods. We used the device to measure the kinetics of extraction of europium ions $\left(\mathrm{C}_{0}=1 \mu \mathrm{M}\right)$ from the aqueous phase (0.05 M HEDTA, $1 \mathrm{M} \mathrm{NaCl}, 0.2 \mathrm{mM}$ lactic acid, $\mathrm{p}[\mathrm{H}]$ 3.00) by $0.01 \mathrm{M}$ HDEHP in dodecane (Figure 6). $k_{a o}$ was determined to be $9.1 \times 10^{-5} \pm 0.8 \times 10^{-5} \mathrm{~cm} / \mathrm{s}$ and $k_{o a}$ was $4.7 \times 10^{-5} \pm 2.2 \times 10^{-5} \mathrm{~cm} / \mathrm{s}$; the literature value, extracted from a graph, of $k_{a o}$ for this extraction was $3 \times 10^{-}$ $5 \mathrm{~cm} / \mathrm{s}$. $\stackrel{46}{ }$ The literature value was obtained using a centimeter-scale Lewis cell; contemporaneous modeling $\frac{15}{}$ predicted that a $60 \mu \mathrm{m}$ diffusion zone was located in the center of that device. Each droplet in the two-phase system tested here was only $\sim 160 \mu \mathrm{m}$ long under tested conditions and $125 \mu \mathrm{m}$ wide, with rapid internal mixing. We would therefore expect to find a higher $k_{a 0}$ than measured previously by constant-interfacial area cells.

\section{TALSPEAK Kinetics Initial Results}

Next, we used the device to test the kinetics of extraction of a mixture of yttrium and the full series of stable lanthanides under TALSPEAK conditions. $C_{0}$ of each cation was $0.01 \mathrm{mM}(0.15 \mathrm{mM}$ total metal ion) in $0.05 \mathrm{M}$ DTPA and $1 \mathrm{M}$ ammonium citrate $(\mathrm{pH}=3.55)$; the organic phase was $1 \mathrm{M}$ HDEHP in $\mathrm{n}$ dodecane. The ambient temperature was $20.0^{\circ} \mathrm{C} \pm 0.5^{\circ} \mathrm{C}$. The organic to aqueous ratio was $1: 2$. The collected aqueous sample stream was analyzed at each time point using ICP-MS to quantify all cations simultaneously (Figure 7), and interfacial mass transport rate constants were determined for each cation (Figure 8, Table 2). The true rate constant should be independent of the initial concentration of lanthanides. We verified that the rate constants remained the same after decreasing the concentration of each cation ten-fold to $0.015 \mathrm{mM}$ (Figure 8). These results indicated that $\mathrm{Dy}^{3+}$ has the slowest extraction kinetics under the TALSPEAK conditions tested. 

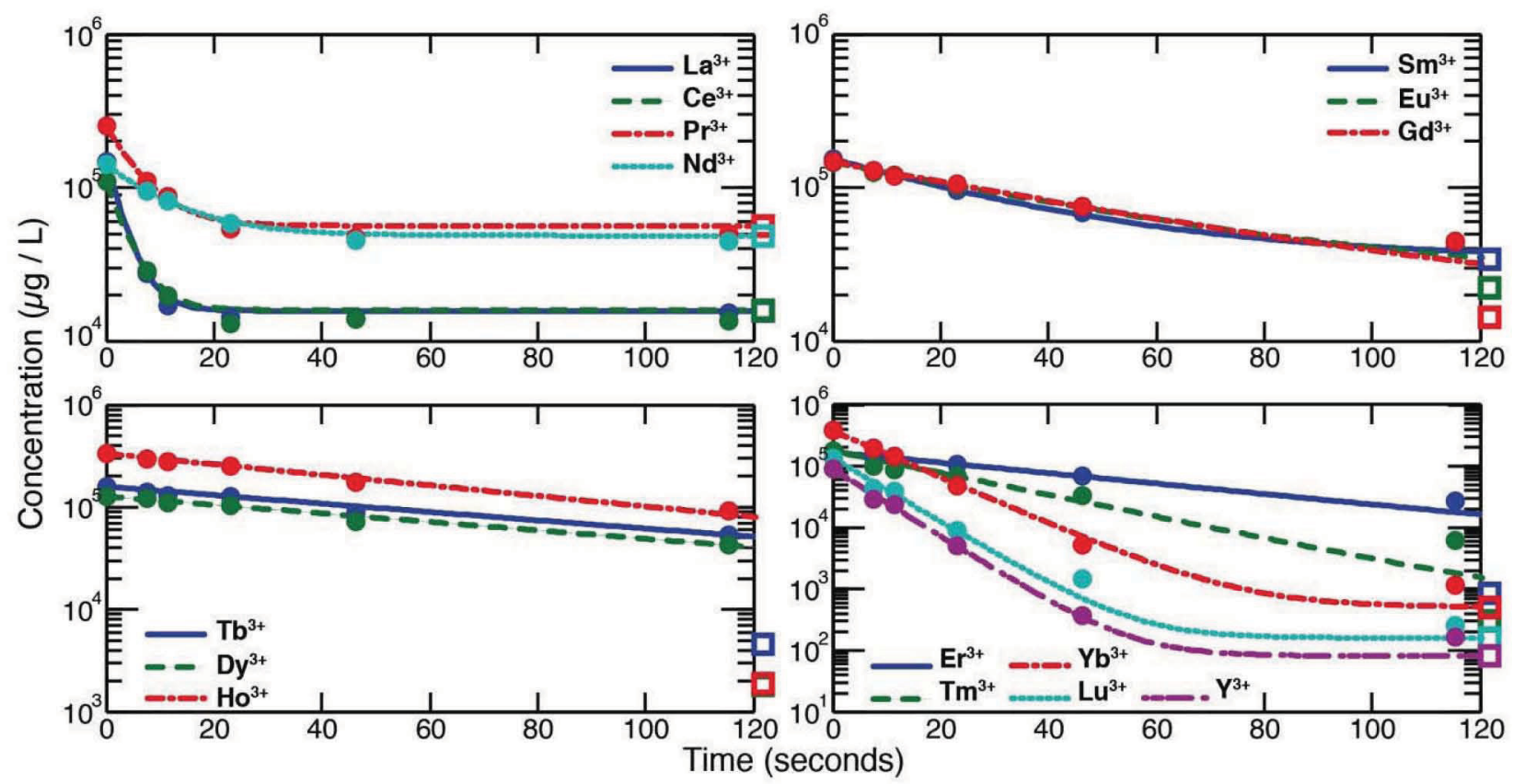

Figure 7. Plots of concentration vs. time for extraction obtained using the tested device under TALSPEAK conditions: the aqueous phase was $0.05 \mathrm{M}$ DTPA and $1 \mathrm{M}$ ammonium citrate $(\mathrm{pH}=3.55)$; the organic phase was $1 \mathrm{M}$ HDEHP in n-dodecane. Organic to aqueous ratio was 1:2. The ambient temperature was $20.0^{\circ} \mathrm{C} \pm 0.5^{\circ} \mathrm{C}$. The first 120 seconds of primary data is plotted. This data were used to obtain the constants in Table 2, plotted in Figure 8. Circles indicate primary data points obtained using the tested device and ICP-MS. Squares indicate equilibrium points, obtained via test-tube experiments and identical ICP-MS processing. The curves were fit using Eq. 7.

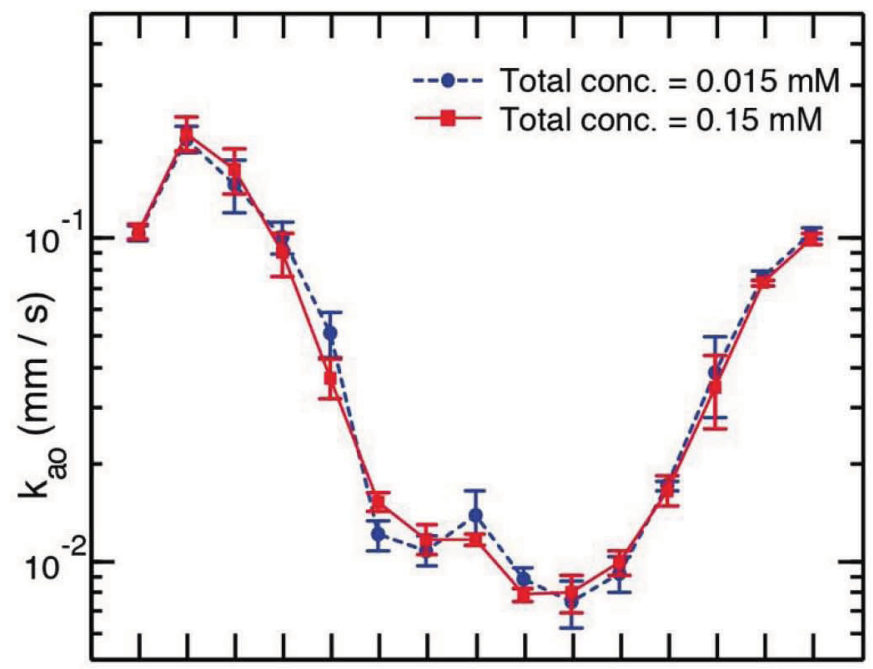

3+: Y La Ce Pr NdSmEu Gd Tb Dy Ho Er Tm Yb Lu

Figure 8. Kinetic data obtained using the device under TALSPEAK conditions. $\mathrm{C}_{0}$ of each cation was either $0.01 \mathrm{mM}$ (red squares) or $0.001 \mathrm{mM}$ (blue circles) in $0.05 \mathrm{M}$ DTPA and $1 \mathrm{M}$ ammonium citrate $(\mathrm{pH}=3.55)$; the organic phase was $1 \mathrm{M}$ HDEHP in n-dodecane. The organic to aqueous ratio was $1: 2$. The ambient temperature was $20.0{ }^{\circ} \mathrm{C} \pm 0.5^{\circ} \mathrm{C}$. A plot of the $k_{a o}$ obtained from each regression for each cation. The error bar on each $k_{a o}$ indicates the $95 \%$ confidence interval. Error bars were calculated from single experiments (Figure 7), and indicate the confidence interval calculated based upon the deviation from the fit to individual measurements of concentration vs. time. Such error bars were determined in 
MATLAB 7.6 using the inverse $\mathrm{R}$ factor from the $\mathrm{QR}$ decomposition of the Jacobian, the degrees of freedom for error, and the root mean squared error. Table 2 presents $k_{a o}$ and $k_{o a}$ values corresponding to the red curve.

Table 2. Kinetics obtained using the device under TALSPEAK conditions, for the conditions shown as a graph in Figure $8 . \mathrm{C}_{0}$ of each cation was $0.01 \mathrm{mM}$ in $0.05 \mathrm{M}$ DTPA and $1 \mathrm{M}$ ammonium citrate, $\mathrm{pH}=$ 3.55; the organic phase was $1 \mathrm{M}$ HDEHP in $\mathrm{n}$-dodecane. The organic to aqueous ratio was $1: 2$. The ambient temperature was $20.0^{\circ} \mathrm{C} \pm 0.5^{\circ} \mathrm{C}$.

\begin{tabular}{|l|l|l|}
\hline Cation & $\mathbf{k}_{\mathbf{a}}(\mathbf{m m} / \mathbf{s})$ & $\mathbf{k}_{\mathbf{0 a}}(\mathbf{m m} / \mathbf{s})$ \\
\hline $\mathbf{Y}$ & $1.0 \times 10^{-01} \pm 5.5 \times 10^{-03}$ & $1.9 \times 10^{-04} \pm 1.0 \times 10^{-05}$ \\
\hline La & $2.1 \times 10^{-01} \pm 2.6 \times 10^{-02}$ & $5.0 \times 10^{-02} \pm 6.1 \times 10^{-03}$ \\
\hline Ce & $1.6 \times 10^{-01} \pm 2.6 \times 10^{-02}$ & $5.5 \times 10^{-02} \pm 8.8 \times 10^{-03}$ \\
\hline Pr & $9.0 \times 10^{-02} \pm 1.4 \times 10^{-02}$ & $5.2 \times 10^{-02} \pm 7.9 \times 10^{-03}$ \\
\hline Nd & $3.7 \times 10^{-02} \pm 5.3 \times 10^{-03}$ & $3.9 \times 10^{-02} \pm 5.6 \times 10^{-03}$ \\
\hline Sm & $1.5 \times 10^{-02} \pm 9.6 \times 10^{-04}$ & $8.7 \times 10^{-03} \pm 5.5 \times 10^{-04}$ \\
\hline Eu & $1.2 \times 10^{-02} \pm 1.3 \times 10^{-03}$ & $4.2 \times 10^{-03} \pm 4.5 \times 10^{-04}$ \\
\hline Gd & $1.2 \times 10^{-02} \pm 4.7 \times 10^{-04}$ & $2.5 \times 10^{-03} \pm 1.0 \times 10^{-04}$ \\
\hline Tb & $7.8 \times 10^{-03} \pm 3.8 \times 10^{-04}$ & $4.6 \times 10^{-04} \pm 2.2 \times 10^{-05}$ \\
\hline Dy & $7.9 \times 10^{-03} \pm 1.1 \times 10^{-03}$ & $2.3 \times 10^{-04} \pm 3.1 \times 10^{-05}$ \\
\hline Ho & $9.9 \times 10^{-03} \pm 8.6 \times 10^{-04}$ & $1.1 \times 10^{-044} \pm 9.9 \times 10^{-06}$ \\
\hline Er & $1.6 \times 10^{-02} \pm 1.7 \times 10^{-03}$ & $1.7 \times 10^{-04} \pm 1.7 \times 10^{-05}$ \\
\hline Tm & $3.4 \times 10^{-02} \pm 8.9 \times 10^{-03}$ & $1.2 \times 10^{-04} \pm 3.1 \times 10^{-05}$ \\
\hline Yb & $7.2 \times 10^{-02} \pm 1.7 \times 10^{-03}$ & $1.9 \times 10^{-04} \pm 4.5 \times 10^{-06}$ \\
\hline Lu & $9.9 \times 10^{-02} \pm 3.8 \times 10^{-03}$ & $2.3 \times 10^{-04} \pm 8.8 \times 10^{-06}$ \\
\hline
\end{tabular}

In a mixture of cations, the components could interfere with one another; for example, if more rapidlyextracted cations dominate the available interfacial HDEHP binding sites, thereby slowing the extraction of other cations. We tested whether interference affected the kinetics observed in the device by measuring the interfacial mass transfer rate constant of the metal ions with the lowest rate constant, $\mathrm{Dy}^{3+}$, alone instead of in a mixture. $C_{0}$ of each cation was $0.01 \mathrm{mM}$ in $0.05 \mathrm{M}$ DTPA and $1 \mathrm{M}$ ammonium citrate $(\mathrm{pH}$ = 3.55); the organic phase was $1 \mathrm{M}$ HDEHP in n-dodecane. The organic to aqueous ratio was $1: 2$. The ambient temperature was $20.0{ }^{\circ} \mathrm{C} \pm 0.5^{\circ} \mathrm{C}$. We found the rate constant was unchanged (Table 3); therefore, cation interference is not a significant effect in this system, as expected from the high HDEHP:metal ion molar ratio.

Table 3. Interfacial mass transfer rate constants for dysprosium in a mixture of 14 other metal cations and without additional metal cations. Error bars were calculated after non-linear regression to Eq. 7 and indicate the confidence interval calculated based upon the deviation from the fit to individual measurements.

\begin{tabular}{|l|l|}
\hline & $k_{a 0}(\mathrm{~mm} / \mathrm{s})$ \\
\hline $\mathrm{Dy}^{3+}$ (in lanthanide mixture) & $0.0083 \pm 0.0016$ \\
\hline $\mathrm{Dy}^{3+}$ (without lanthanide mixture) & $0.0091 \pm 0.0010$ \\
\hline
\end{tabular}




\section{Conclusions}

Here, we have tested a microfluidic method to measure the kinetics of metal ion extraction under conditions that are in development for separation of streams of used nuclear fuel. This device, for the first time, measured individual absolute interfacial mass transport rate constants for 15 nuclides extracted from a mixture while surface area, mixing rate, and other parameters were under total control. Furthermore, the high specific interfacial area enabled rapid rates of reaction, which is especially valuable for reactions with high rate constants because it allows i) measurement of the extent of reaction from short contact times to equilibrium; and ii) the measurement of true rate constants was not obscured by mixing limitations. For a series of experiments such as in Figures 7 and 8 , each time point required $10 \mu \mathrm{L}$ of aqueous phase for ICP-MS analysis (which produced data for all metal ions at each time point), and produced a total of less than $30 \mu \mathrm{L}$ of liquid waste (total of aqueous and organic), therefore addressing the significant experimental radiation safety concerns.

The data obtained by this method revealed unexpected kinetic information that is relevant to mechanistic insight and industrial models of the TALSPEAK process. We observed that $\mathrm{Dy}^{3+}$ has the slowest extraction kinetics under the TALSPEAK conditions, and that the heavier lanthanides are actually extracted faster under the tested conditions. Previously, it was believed that the extraction rate slows down as the lanthanide atomic number increases, ${ }^{49}$ but the heavy lanthanides (beyond Gd) were not probed in previous studies. The extraction mechanism of TALSPEAK includes several steps that could affect the overall rate of interfacial mass transfer, such as decomplexation of the Ln-DTPA complex, transfer of $\mathrm{Ln}^{3+}$ to the interface (believed to be rapid in the device tested here), and multi-step formation of the Ln-HDEHP complex in the organic phase. ${ }^{14}$ The buffer (here citrate, though lactate is commonly used as well) may also play a role in determining the rate of extraction, which requires further investigation. Further mechanistic understanding and optimization of TALSPEAK chemistry and its analogues will be greatly enhanced by this methodology, facilitating reprocessing of nuclear waste with the potential to address a problem of significance to energy, environment, and national security.

\section{Indicators of Project Quality}

The results of this project were recently reported in the Journal of the American Chemical Society under the title "Toward Mechanistic Understanding of Nuclear Reprocessing Chemistries by Quantifying Lanthanide Solvent Extraction Kinetics via Microfluidics with Constant Interfacial Area and Rapid Mixing." This publication is available online, as of September 2, 2011, at the following address: http://dx.doi.org/10.1021/ja206020u

Additionally, this project was accepted for a presentation at the $15^{\text {th }}$ International Conference on Miniaturized Systems for Chemistry and Life Sciences.

\section{References}

(1) Crowley, K. D. Phys. Today 1997, 50, 32-39.

(2) Todd, T. A.; Wigeland, R. A. In Separations for the Nuclear Fuel Cycle in the 21st Century; American Chemical Society: 2006; Vol. 933, p 41-55.

(3) Fukasawa, T.; Yamashita, J.; Hoshino, K.; Fujimura, K.; Sasahira, A. In ASME Conference Proceedings Orlando, Florida, 2008; Vol. 2008, p 105-109.

(4) Andrews, A. Nuclear Fuel Reprocessing: US Policy Development, Congressional Research Service, 2006.

(5) Gelis, A. V.; Vandegrift, G. F.; Bakel, A.; Bowers, D. L.; Hebden, A. S.; Pereira, C.; Regalbuto, M. Radiochim. Acta 2009, 97, 231-232. 
(6) Vandegrift, G. F., et al. In Advances for Future Nuclear Fuel Cycles International Conference Nimes, France, 2004.

(7) Vandegrift, G. F., et al. In Waste Management '04 Conference Tucson, AZ, 2004.

(8) Nash, K. L. Solvent Extr. Ion Exc. 1993, 11, 729-768.

(9) Nash, K. L. Key features of the TALSPEAK and similar trivalent actinide-lanthanide partitioning processes, Chemical Sciences Division, Oak Ridge National Laboratory (United States), 2008.

(10) Nilsson, M.; Nash, K. L. Solvent Extr. Ion Exc. 2007, 25, 665-701.

(11) Nilsson, M.; Nash, K. L. Solvent Extr. Ion Exc. 2009, 27, 354-377.

(12) Grimes, T. S.; Nilsson, M.; Nash, K. L. The behavior and importance of lactic acid complexation in Talspeak extraction systems, Chemical Sciences Division, Oak Ridge National Laboratory (United States), 2008.

(13) Zalupski, P. R.; Nash, K. L.; Nakamura, Y.; Yamamoto, M.; Martin, L. R. In IOP Conf. Ser.: Mater. Sci. Eng. 2010; Vol. 9, p 012076.

(14) Danesi, P. R.; Chiarizia, R.; Coleman, C. Crit. Rev. Anal. Chem. 1980, 10, 1-126.

(15) Danesi, P. R.; Vandegrift, G. F.; Horwitz, E. P.; Chiarizia, R. J. Phys. Chem. 1980, 84, 3582-

3587.

(16) Kubán, V. Crit. Rev. Anal. Chem. 1991, 22, 477-477.

(17) Song, H.; Chen, D. L.; Ismagilov, R. F. Angew. Chem. Int.-Ed. 2006, 45, 7336-7356.

(18) Bringer, M. R.; Gerdts, C. J.; Song, H.; Tice, J. D.; Ismagilov, R. F. Philos. T. R. Soc. A. 2004, $362,1087-1104$.

(19) Song, H.; Bringer, M. R.; Tice, J. D.; Gerdts, C. J.; Ismagilov, R. F. Appl. Phys. Lett. 2003, 83, 4664-4666.

(20) Tice, J. D.; Song, H.; Lyon, A. D.; Ismagilov, R. F. Langmuir 2003, 19, 9127-9133.

(21) Song, H.; Li, H. W.; Munson, M. S.; Van Ha, T. G.; Ismagilov, R. F. Anal. Chem. 2006, 78, 4839-4849.

(22) Chen, D. L.; Gerdts, C. J.; Ismagilov, R. F. J. Am. Chem. Soc. 2005, 127, 9672-9673.

(23) Song, H.; Ismagilov, R. F. J. Am. Chem. Soc. 2003, 125, 14613-14619.

(24) Kreutz, J. E.; Shukhaev, A.; Du, W.; Druskin, S.; Daugulis, O.; Ismagilov, R. F. J. Am. Chem. Soc. 2010, 132, 3128-3132.

(25) Begolo, S.; Colas, G.; Viovy, J. L.; Malaquin, L. Lab Chip 2011, 11, 508-512.

(26) Kralj, J. G.; Sahoo, H. R.; Jensen, K. F. Lab Chip 2007, 7, 256-263.

(27) Li, L.; Boedicker, J. Q.; Ismagilov, R. F. Anal. Chem. 2007, 79, 2756-2761.

(28) Kralj, J. G.; Schmidt, M. A.; Jensen, K. F. Lab Chip 2005, 5, 531-535.

(29) Kreutzer, M. T.; Gunther, A.; Jensen, K. F. Anal. Chem. 2008, 80, 1558-1567.

(30) Mary, P.; Studer, V.; Tabeling, P. Anal. Chem. 2008, 80, 2680-2687.

(31) Priest, C.; Zhou, J.; Sedev, R.; Ralston, J.; Aota, A.; Mawatari, K.; Kitamori, T. Int. J. Miner. Process. 2011, 98, 168-173.

(32) Silvestre, C. I. C.; Santos, J. L. M.; Lima, J. L. F. C.; Zagatto, E. A. G. Anal. Chim. Acta 2009, $652,54-65$.

(33) Abate, A. R.; Thiele, J.; Weitz, D. A. Lab Chip 2011, 11, 253-258.

(34) Anna, S. L.; Bontoux, N.; Stone, H. A. Appl. Phys. Lett. 2003, 82, 364-366.

(35) Utada, A. S.; Lorenceau, E.; Link, D. R.; Kaplan, P. D.; Stone, H. A.; Weitz, D. A. Science 2005, $308,537-541$.

(36) Teh, S. Y.; Lin, R.; Hung, L. H.; Lee, A. P. Lab Chip 2008, 8, 198-220.

(37) Tice, J. D.; Lyon, A. D.; Ismagilov, R. F. Anal. Chim. Acta 2004, 507, 73-77.

(38) Abate, A. R.; Poitzsch, A.; Hwang, Y.; Lee, J.; Czerwinska, J.; Weitz, D. A. Phys. Rev. E 2009, 80

(39) Handique, K.; Burns, M. A. J. Micromech. Microeng. 2001, 11, 548-554. 
(40) Kashid, M. N.; Gerlach, I.; Goetz, S.; Franzke, J.; Acker, J. F.; Platte, F.; Agar, D. W.; Turek, S. Ind. Engr. Chem. Res. 2005, 44, 5003-5010.

(41) Rhee, M.; Burns, M. A. Langmuir 2008, 24, 590-601.

(42) King, C.; Walsh, E.; Grimes, R. Microfluid. Nanofluid. 2007, 3, 463-472.

(43) Song, H.; Chen, D. L.; Ismagilov, R. F. Angew. Chem.-Int. Edit. 2006, 45, 7336-7356.

(44) Scheiff, F.; Mendorf, M.; Agar, D.; Reis, N.; Mackley, M. Lab Chip 2011, 11, 1022-1029.

(45) Li, L.; Karymov, M. A.; Nichols, K. P.; Ismagilov, R. F. Langmuir 2010, 26, 12465-12471.

(46) Danesi, P. R.; Cianetti, C. Separ. Sci. Technol. 1982, 17, 969-969.

(47) Danesi, P. R.; Vandegrift, G. F. J. Phys. Chem. 1981, 85, 3646-3651.

(48) Ting, H. P.; Bertrand, G. L.; Sears, D. F. Biophys. J. 1966, 6, 813-823.

(49) Kolarik, Z.; Koch, G.; Kuhn, W. J Inorg Nuc Chem 1974, 36, 905-909. 


\title{
Appendix C
}

\section{Electrochemical and Computation Investigation of the TALSPEAK Processes}

\author{
Dr. Nicholas Bridges
}

Savannah River National Laboratory 


\section{SUMMARY}

As a viable scheme to separate the trivalent actinides from the lanthanides, the TALSPEAK process (Trivalent Actinide-Lanthanide Separation by Phosphorous-reagent Extraction from Aqueous Komplexes) has shown the most promising results, yet is still not fully understood. For implementation of the TALSPEAK process all possible deviations must be understood (e.g., any breakthrough of plutonium from the head end process) and a better understanding of the chemistry which drives the separation of the $\mathrm{Ln}^{\text {III }}$ from the $\mathrm{An}^{\mathrm{III}}$. The majority of published TALSPEAK work is based on measuring the $\mathrm{Ln}^{\text {III }} / \mathrm{An}^{\mathrm{III}}$ separation factor with less research on plutonium interactions or the theoretical basis of the $\mathrm{Ln}^{\text {III }} / \mathrm{An}^{\text {III }}$ separation. Here, the separation is explored through electrochemical methods to examine the relative log $\beta$ as a function of plutonium's oxidation state, and through computational modeling to better understand the thermodynamics of DTPA interactions with $\mathrm{Ln}^{\mathrm{III}}$ and $\mathrm{An}{ }^{\mathrm{III}}$.

The Pu-DTPA complex favors the same coordination environment with both $\mathrm{Pu}^{\mathrm{III}}$ and $\mathrm{Pu}^{\mathrm{IV}}$ in the $\mathrm{pH}$ range investigated here. The ratio of the $\log \beta$ for $\mathrm{Pu}^{\mathrm{IV}}$ to $\mathrm{Pu}^{\mathrm{III}}$ is constant over the investigated $\mathrm{pH}$ range, supporting that the same coordination environment is preferred by both oxidation states in the Pu-DTPA complex.

Computational theory is able to predict the experimental observation that $\mathrm{Am}^{\mathrm{III}}$ complexation with the $\mathrm{DTPA}^{5-}$ ligand is preferred over $\mathrm{Nd}$ by $\sim 1 \mathrm{kcal} \mathrm{mol}^{-1}$. The larger radial extent of the $5 f$ orbitals causes an increase in the covalency of the bonds, and a preference for the oxygen chelates.

\section{INTRODUCTION}

The separation of transplutonium elements from the lanthanide series may be accomplished with the Trivalent Atinide-Lanthanide Separations by Phosphorous-reagent Extraction from Aqueous Komplexes (TALSPEAK) processes. ${ }^{1,2}$ Separation of transplutonium elements from lanthanides is complicated by the similar liquid extraction behavior of americium, curium, and the lanthanides, due to their similar size and predominance in the +3 oxidation state. For the TALSPEAK process to become viable on a production scale, a stronger fundamental understanding of all the components and how they interact is needed.

From the pilot scale demonstration of the TALSPEAK process in a closed fuel cycle conducted at Oak Ridge National Laboratory (ORNL) an unexpected result was reported. In the TALSPEAK processes conducted at ORNL, significant amounts of early actinides (i.e., uranium, $4.3 \mathrm{~g}$; neptunium, $0.23 \mathrm{~g}$; and plutonium, $0.91 \mathrm{~g}$ ) were present in the feed stream, and thus were carried through the TALSPEAK process. ${ }^{3}$

In this study, electrochemical techniques were used to investigate the aqueous phases of diethylenetriamine-N,N,N',N",N"'-pentaacetic acid (DTPA) with plutonium, to better understand the TALSPEAK process's interactions with the actinides. Experimental work was also complimented by the addition of computational modeling investigating the strength of the coordination of DTPA with a lanthanide and americium.

\section{SIGNIFICANCE}

Today, most of the chemistry studies performed on the TALSPEAK processes have centered on the separation of transplutonium and lanthanide elements. The most recent pilot process of a closed fuel cycle was successful, but not without some interesting results. One of these results 
was the presence of uranium, neptunium, and plutonium carryover into the TALSPEAK processes. Though neptunium and plutonium followed the americium and curium, their interactions with the TALSPEAK processes are not as well studied as americium. Uranium was extracted by the organic phase and was unable to be stripped due to the strong interactions of uranium with bis-(2-ethylhexyl) phosphoric acid. It is well known that the transplutonium elements are not easily oxidized/reduced, and thus are found in solution in the +3 oxidation state. However, plutonium has a wide range of oxidation potentials in aqueous systems thus making its chemistry more complicated. Understanding the chemical and oxidation stabilities of the TALSPEAK process will benefit further research and operation of the TALSPEAK process. Computational work can provide a gateway into understanding the chemistry of the TALSPEAK process based around the fundamental thermodynamics of the system.

\section{APPROACH}

Details of the electrochemical experiments and the preparation of the stock solutions have been provided in previous reports. ${ }^{4}$ Spectroscopic measurements were conducted using fiber optic cables which transmit the source light (tungsten filament) into the glovebox, and which returns the transmitted light to the detector. The detector is a AvaSpec that was calibrated with a $\mathrm{Ar}(\mathrm{Hg})$ source.

Computational modeling was conducted using with Gaussian 09 (Version B.01), additional details about the computational methods are provided under Section 8.

\section{SUMMARY OF RESULTS}

\section{Electrochemical Method}

As previously reported, ${ }^{4}$ DTPA is present predominantly as three different species in the investigated $\mathrm{pH}$ range (3.50-4.00); the speciation diagram of DTPA is shown in Figure 1. Because electrochemical proton exchange is too fast for any of the reported techniques to resolve, it can not be determined which carboxylic acid groups are protonated, and thus only one averaged species is observed in the CV shown in Figure 2. The amplitude of the reduction current for the change from a carboxylic acid to an aldehyde is related to the amount, or chemical activity, of the reduced species. The change in the reduction potential as a function of $\mathrm{pH}$ from -0.6 to $-0.7 \mathrm{~V}$ vs $\mathrm{Ag} / \mathrm{AgCl}$ is related to the changes in DTPA speciation. As the $\mathrm{pH}$ changes from $\mathrm{pH} 3.5$ to $\mathrm{pH} 4.0$ a decrease in the concentration of mono-deprotonated DTPA $\left(\mathrm{DTPAH}_{4}^{-}\right)$is observed concurrent with an increase in the concentration of tri-deprotonated DTPA $\left(\mathrm{DTPAH}_{2}{ }^{-3}\right)$. The relative amount of the di-deprotonated DTPA $\left(\mathrm{DTPAH}_{3}{ }^{-2}\right)$ is less affected by $\mathrm{pH}$ changes in this window, as it is always the predominant species with minimal changes in concentration within the investigated $\mathrm{pH}$ range.

The presence of multiple DTPA species complicates the analysis of the system, as it is impossible to know for sure which species is chelating the plutonium. This complex system is also most likely the reason why the reduction of the $\mathrm{Pu}^{\mathrm{IV}}$-DTPA complex is quasi-reversible with a significant effect due to scan rate and $\mathrm{pH}$ as shown in Figure 3. In Table 1, the slower the scan rate, the more reversible the $\mathrm{Pu}^{\text {IV/III }}$ couple. This increase in reversibility can be attributed to providing enough time for the $\left[\mathrm{DPTAH}_{\mathrm{x}}-\mathrm{Pu}^{\mathrm{IV}}\right]^{\mathrm{m}+}$ to change to $\left[\mathrm{DTPAH}_{\mathrm{y}}-\mathrm{Pu}^{\mathrm{III}}\right]^{\mathrm{n}+}$ (where $\mathrm{x} \& \mathrm{y}$ are $2-4$ and $m \& n$ are $0-3$ ). If the DTPA-Pu complex has sufficient time to revert (i.e., removal or addition of a proton to the DTPA complex) back to the original orientation before the 
subsequent $\mathrm{CV}$ sweep, the $\mathrm{Pu}^{\text {IV/III }}$ couple would appear to be reversible. Control of time is achieved through changing the scan rate; a slower scan rate means more time is available for reorientation.

There is a general trend of greater electrochemical reversibility with higher $\mathrm{pH}$. Overall, the lower the $\mathrm{pH}(3.50)$, the larger the difference between observed oxidation and reduction peaks indicative of a more electrochemically irreversible $\mathrm{Pu}^{\text {IV/III }}$ couple. Higher $\mathrm{pHs}$ of 3.75 and 4.00 display a more electrochemically reversible $\mathrm{Pu}^{\mathrm{IV} / I I I}$ couple, with a difference between peaks closer to the ideal $58 \mathrm{mV}$. The largest difference in the DTPA systems, between $\mathrm{pH} 3.50$ and 3.75 , is most likely due to the relative concentration of the $\mathrm{DTPAH}_{2}{ }^{3-}$, which increases significantly in this $\mathrm{pH}$ window. The change in the relative concentration of $\mathrm{DTPAH}_{2}{ }^{3-}$ is less between $\mathrm{pH} 3.75$ and 4.00. Currently, no other evidence exists to confirm this theory, but it does support the observed changes in reversibility.

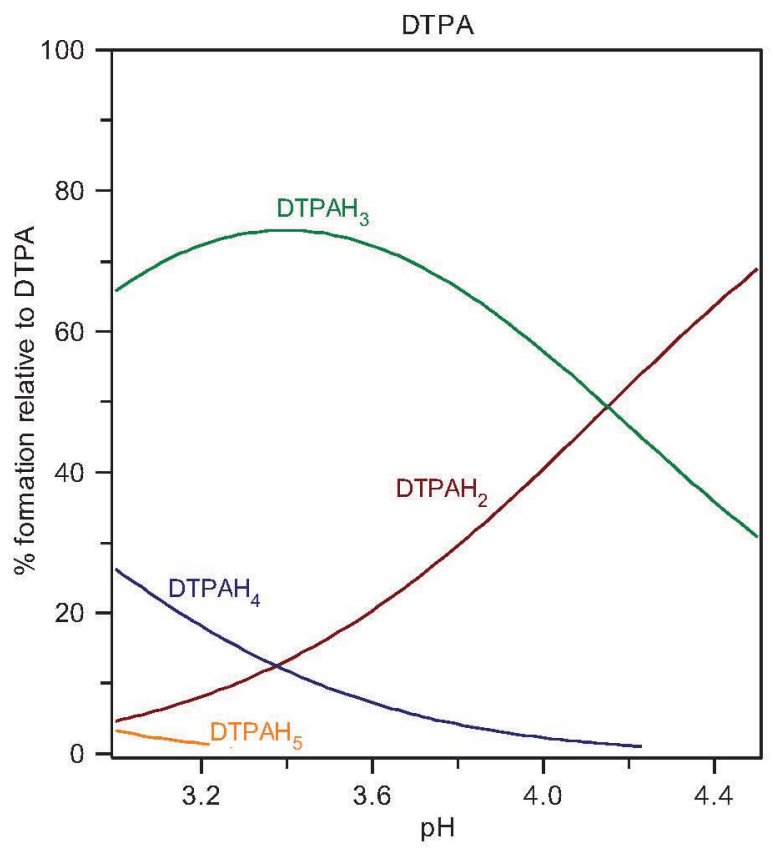

Figure 1. Speciation of DTPA in investigated $\mathrm{pH}$ range. DTPA $\mathrm{pK}_{\mathrm{A}}(\mathrm{DTPA})=1.68,2.1,2.6,4.15,8.2,9.9$. 


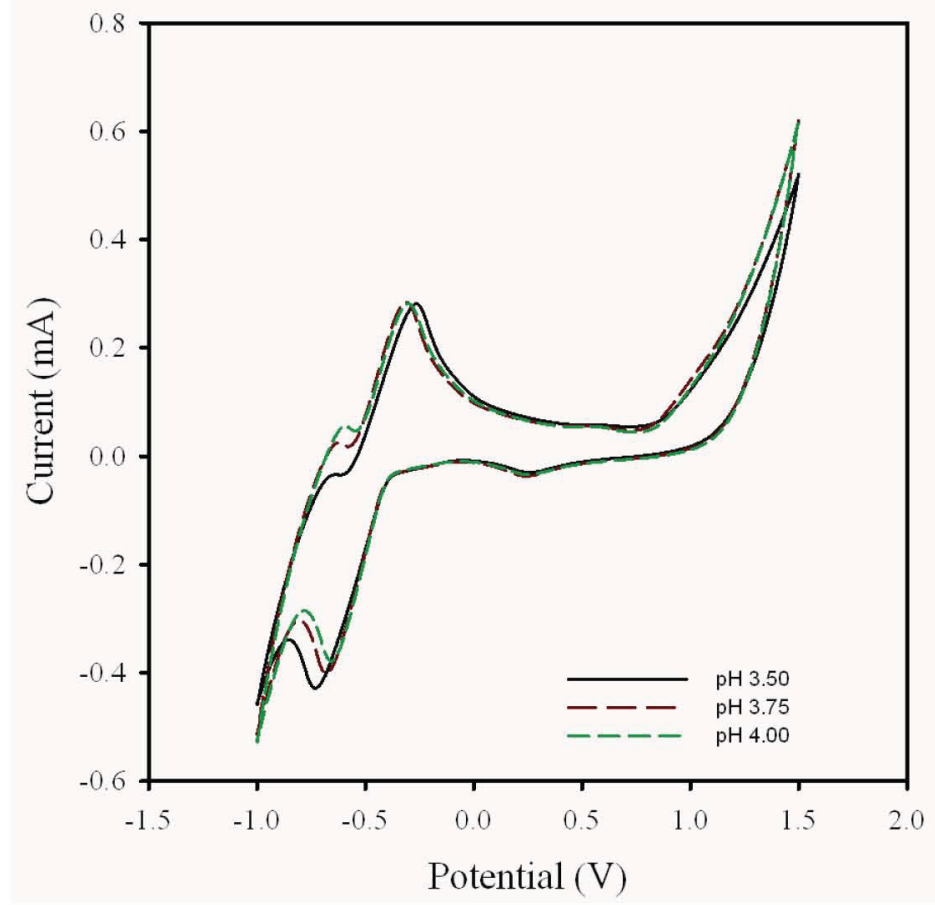

Figure 2. Cyclic voltammetry of DTPA as a function of $\mathrm{pH}$

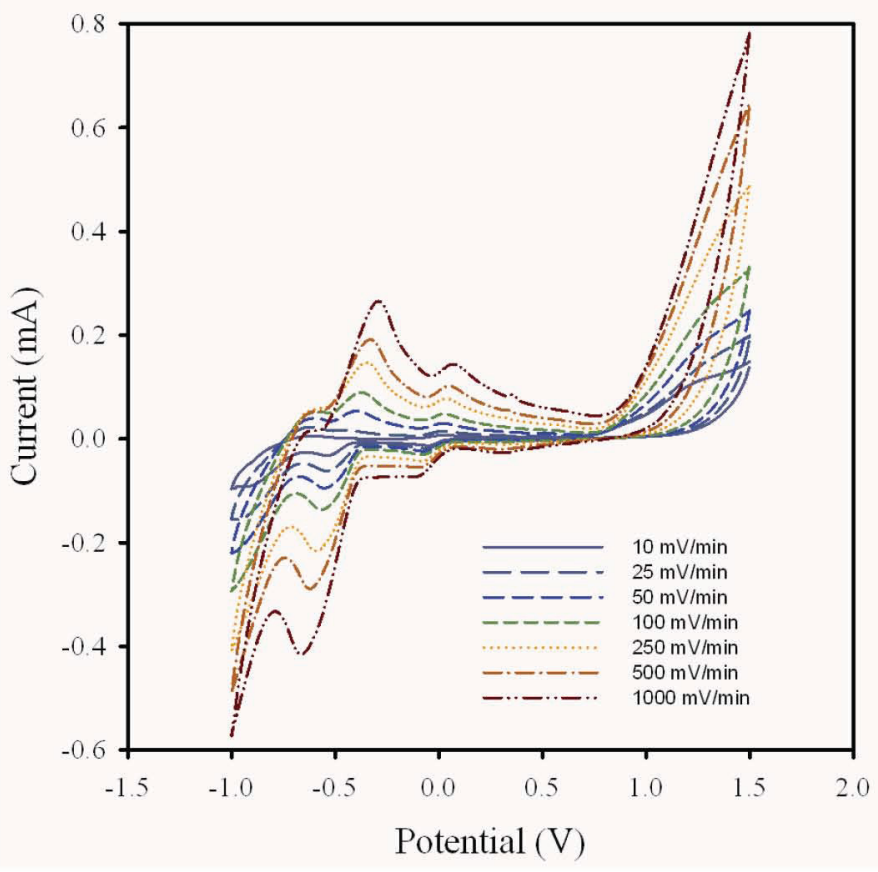

Figure 3. Cyclic voltametery of $50 \mathrm{mM}$ DTPA in $1.0 \mathrm{M} \mathrm{NaClO}_{4}$ with variable scan rates with $7.92 \mathrm{mM} \mathrm{Pu}, \mathrm{pH}$ $3.75, \mathrm{Pt} / / \mathrm{Pt} / / \mathrm{Ag} / \mathrm{AgCl}$. 
Table 1. Comparison of electrochemical reversibility (electrochemically reversible system is $58 \mathrm{mV}$ ) of $\mathrm{Pu}^{\mathrm{III} / \mathrm{V}}$ as a function of DTPA concentration and $\mathrm{pH}$.

\begin{tabular}{|c|c|c|c|c|c|c|c|c|c|}
\hline & \multicolumn{3}{|c|}{$\mathrm{pH}=3.50$} & \multicolumn{3}{c|}{$\mathrm{pH}=3.75$} & \multicolumn{3}{c|}{$\mathrm{pH}=4.0$} \\
\hline $\begin{array}{c}\text { Scan } \\
\text { Rate } \\
(\mathrm{mV} / \mathrm{S})\end{array}$ & $\begin{array}{c}25 \mathrm{mM} \\
\text { DTPA } \\
(3.2: 1) \\
(\mathrm{mV})\end{array}$ & $\begin{array}{c}50 \mathrm{mM} \\
\text { DTPA } \\
(6.3: 1) \\
(\mathrm{mV})\end{array}$ & $\begin{array}{c}75 \mathrm{mM} \\
\text { DTPA } \\
(9.5: 1) \\
(\mathrm{mV})\end{array}$ & $\begin{array}{c}25 \mathrm{mM} \\
\text { DTPA } \\
(3.2: 1) \\
(\mathrm{mV})\end{array}$ & $\begin{array}{c}50 \mathrm{mM} \\
\text { DTPA } \\
(6.3: 1) \\
(\mathrm{mV})\end{array}$ & $\begin{array}{c}75 \mathrm{mM} \\
\text { DTPA } \\
(9.5: 1) \\
(\mathrm{mV})\end{array}$ & $\begin{array}{c}25 \mathrm{mM} \\
\text { DTPA } \\
(3.2: 1) \\
(\mathrm{mV})\end{array}$ & $\begin{array}{c}50 \mathrm{mM} \\
\text { DTPA } \\
(6.3: 1) \\
(\mathrm{mV})\end{array}$ & $\begin{array}{c}75 \mathrm{mM} \\
\text { DTPA } \\
(9.5: 1) \\
(\mathrm{mV})\end{array}$ \\
\hline 10 & 64 & 76 & 94 & 63 & 76 & 73 & 61 & 66 & 88 \\
\hline 25 & 85 & 78 & 98 & 81 & 84 & 84 & 82 & 81 & 87 \\
\hline 50 & 97 & 88 & 118 & 98 & 88 & 90 & 104 & 85 & 103 \\
\hline 100 & 128 & 103 & 113 & 119 & 108 & 101 & 114 & 110 & 118 \\
\hline 250 & 136 & 123 & 144 & 143 & 121 & 125 & 137 & 122 & 133 \\
\hline 500 & 146 & 158 & 191 & 151 & 150 & 155 & 145 & 151 & 145 \\
\hline 1000 & 178 & 205 & 229 & 183 & 191 & 166 & 180 & 172 & - \\
\hline
\end{tabular}



Table 2. Ratio of $\log \beta$ of $\mathrm{Pu}^{\mathrm{IV}}$ to $\mathrm{Pu}{ }^{\mathrm{III}}$ DTPA complex at various $\mathrm{pHs}$ and concentration ranges.

\begin{tabular}{|c|c|c|c|c|c|c|c|}
\hline \multirow[b]{2}{*}{ DTPA } & \multirow[b]{2}{*}{$\begin{array}{l}\text { Scan } \\
\text { Rate } \\
(\mathrm{mV} / \mathrm{s})\end{array}$} & \multicolumn{2}{|c|}{$\mathrm{pH}=3.5$} & \multicolumn{2}{|c|}{$\mathrm{pH}=3.75$} & \multicolumn{2}{|c|}{$\mathrm{pH}=4.0$} \\
\hline & & $\begin{array}{c}E 1 / 2 \\
(\mathrm{Ag} / \mathrm{AgCl}) \\
(\mathrm{mv})\end{array}$ & $\begin{array}{c}\log (\beta \mathrm{Pu}(\mathrm{IV}) / \\
\beta \mathrm{Pu}(\mathrm{III}))\end{array}$ & $\begin{array}{c}E 1 / 2 \\
(\mathrm{Ag} / \mathrm{AgCl}) \\
(\mathrm{mV})\end{array}$ & $\begin{array}{c}\log (\beta \mathrm{Pu}(\mathrm{IV}) / \\
\beta \mathrm{Pu}(\mathrm{III}))\end{array}$ & $\begin{array}{c}E 1 / 2 \\
(\mathrm{Ag} / \mathrm{AgCl}) \\
(\mathrm{mV})\end{array}$ & $\begin{array}{c}\log (\beta \mathrm{Pu}(\mathrm{IV}) / \\
\beta \mathrm{Pu}(\mathrm{III}))\end{array}$ \\
\hline \multirow{7}{*}{$25 \mathrm{mM}$} & 10 & -12 & 15.97 & -9.5 & 15.93 & -11.5 & 15.96 \\
\hline & 25 & -21.5 & 16.13 & -20.5 & 16.11 & -21 & 16.12 \\
\hline & 50 & -25.5 & 16.20 & -27 & 16.23 & -25 & 16.19 \\
\hline & 100 & -34 & 16.34 & -32.5 & 16.32 & -31 & 16.29 \\
\hline & 250 & -30 & 16.28 & -36.5 & 16.38 & -31.5 & 16.30 \\
\hline & 500 & -25 & 16.19 & -23.5 & 16.17 & -23.5 & 16.17 \\
\hline & 1000 & -22 & 16.14 & -25.5 & 16.20 & -26 & 16.21 \\
\hline \multirow{7}{*}{$50 \mathrm{mM}$} & 10 & -24 & 16.17 & -12 & 15.97 & -13 & 16.00 \\
\hline & 25 & -23 & 16.15 & -22 & 16.14 & -18.5 & 16.08 \\
\hline & 50 & -21 & 16.12 & -26 & 16.21 & -25.5 & 16.20 \\
\hline & 100 & -22.5 & 16.14 & -26 & 16.21 & -28 & 16.24 \\
\hline & 250 & -15.5 & 16.03 & -23.5 & 16.17 & -25 & 16.19 \\
\hline & 500 & -19 & 16.09 & -25 & 16.19 & -28.5 & 16.25 \\
\hline & 1000 & -17.5 & 16.06 & -27.5 & 16.23 & -19 & 16.09 \\
\hline \multirow{7}{*}{$75 \mathrm{mM}$} & 10 & -1 & 15.78 & -19.5 & 16.10 & -15 & 16.02 \\
\hline & 25 & -24 & 16.17 & -25 & 16.19 & -29.5 & 16.27 \\
\hline & 50 & -20 & 16.11 & -24 & 16.17 & -34.5 & 16.35 \\
\hline & 100 & -13.5 & 16.00 & -27.5 & 16.23 & -35 & 16.36 \\
\hline & 250 & -18 & 16.07 & -23.5 & 16.23 & -31.5 & 16.30 \\
\hline & 500 & -21.5 & 16.13 & -23.5 & 16.17 & -29.5 & 16.27 \\
\hline & 1000 & -18.5 & 16.08 & -9 & 15.92 & - & - \\
\hline
\end{tabular}

Through additional probing of the shifts in the standard potentials, the difference in the $\log \beta$ for $\mathrm{Pu}^{\mathrm{IV}}$-DTPA and $\mathrm{Pu}^{\mathrm{III}}$-DTPA can be examined. There is no statistical change in the $\log \beta$ for PuDTPA as a function of $\mathrm{pH}$ or concentration, as displayed in Table 2. The ratio is constant around 16.1, favoring the $\mathrm{Pu}^{\mathrm{IV}}$ over the $\mathrm{Pu}{ }^{\mathrm{III}}$, due to the increased charge density of the harder $\mathrm{Pu}^{\mathrm{IV}}$. The lack of change in the $\log \beta$ ratio is supported by the quasi-reversibility observed in the electrochemical reactions. The literature $\log \beta$ for $\mathrm{Pu}^{\mathrm{IV}}-\mathrm{DTPA}^{5-}$ is 29.5 , and $\mathrm{Pu}^{\mathrm{III}}-\mathrm{DTPA}^{5-}$ is $21.5 .^{5}$ It should be noted that the literature values are with DTPA ${ }^{5-}$, and here the predominate species is DTPAH ${ }_{3}^{2-}$. The significant difference in the literature $\log \beta$ and the measured $\log \beta$ is probably related to the difference in charge of the DTPA species in $\mathrm{pH} 3.5-4.0$ range, the TALSPEAK range. The full impact of the difference in $\log \beta$ values because of $\mathrm{pH}$ can not be fully addressed here, as the TALSPEAK process is also affected by the organic phase which is not investigated here.

The reversibility of the $\mathrm{Pu}^{\mathrm{IV} / I I I}$-DTPA complex indicates that the reorientation of the complex is slow on the electrochemical time scale but faster than the UV/vis timescan as seen in Figure 4. Here the bulk electrolysis of $\mathrm{Pu}^{\mathrm{VI}}$-DTPA was reduced electrochemically to Pu ${ }^{\mathrm{III}}$-DTPA (no difference was observed as a function of $\mathrm{pH}$ or DTPA concentration). The spectra shows $\mathrm{Pu}^{\mathrm{IV}}$ being reduced electrochemically to $\mathrm{Pu}$ III as the DTPA complex, which was not possible with chemical reducing agents. ${ }^{6}$ The spectra is similar to the standard for $\mathrm{Pu}^{\mathrm{III}}$, in $1 \mathrm{M} \mathrm{HClO}_{4}$, and the small differences between the spectra are due to the coordination of DTPA with Pu. 


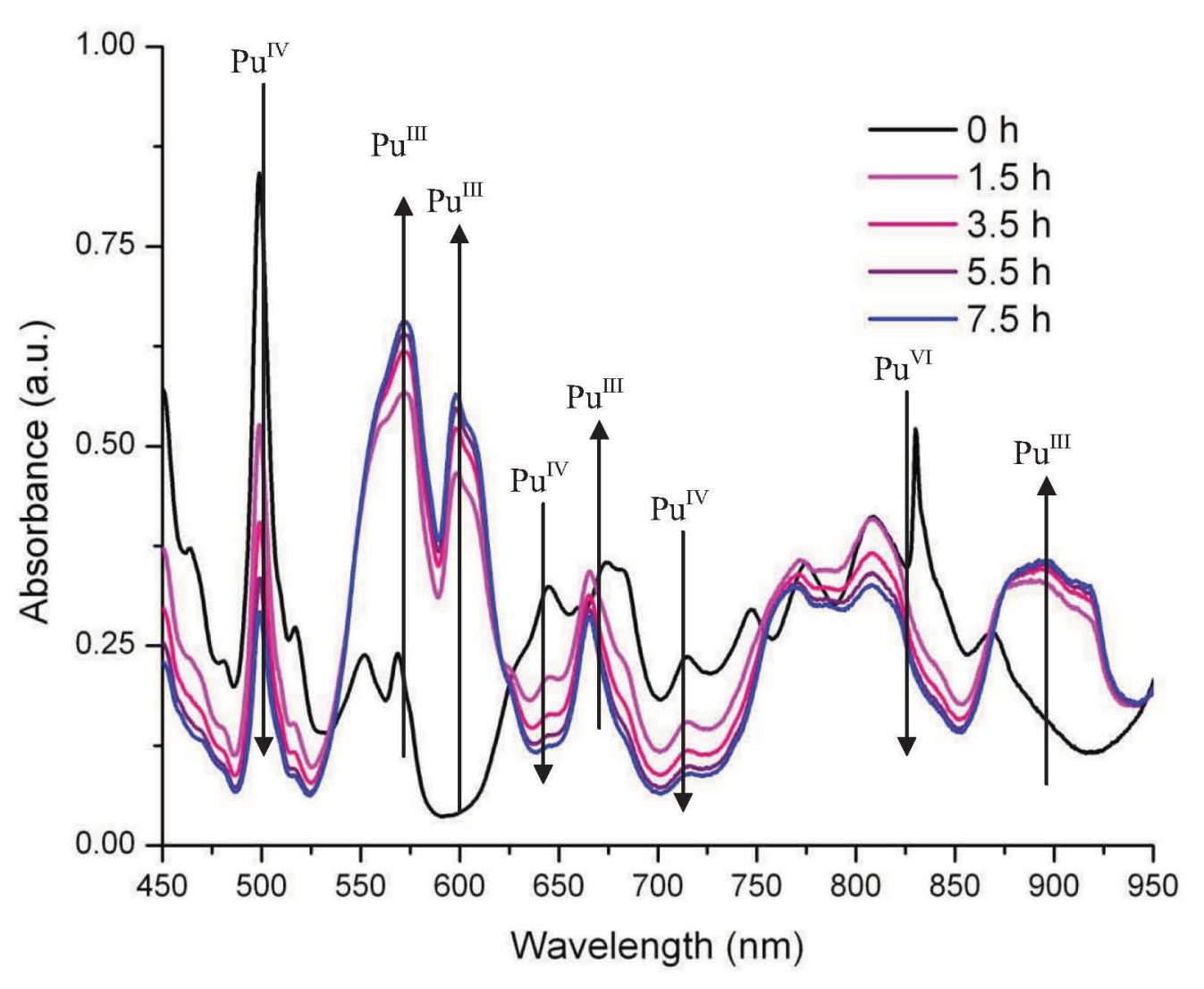

Figure 4. Controlled potential electrolysis reduction of $\mathrm{Pu}^{\mathrm{IV}}$-DTPA to $\mathrm{Pu}$-DTPA, at $\mathrm{pH} 3.75$.

\section{Computational Methods}

\section{Structures of the Complexes [M"'(DTPA)- $\mathrm{H}_{2} \mathrm{O}^{2-}(\mathrm{M}=\mathrm{Nd}, \mathrm{Am})$.}

Selected structural parameters of the optimized structures, as well as available experimental data, are listed in Table $3 .^{7}$ The optimized geometry, as shown using the Nd analogue in Figure 5, reveals that the coordination environment around the metal center can be defined as a distorted monocapped square antiprism. The non-capped base consists of the nitrogen atoms and O1, and the remaining carboxylate oxygen atoms on the ligand form the capped plane wherein an inner sphere water molecule completes the coordination. This structure balances a hydrophilic region at the carboxylate side with a hydrophobic region at the ligand backbone. Compared to experimental data, the calculated bond lengths are marginally longer (by $\leq 0.1 \AA$ ) but still within the standard deviation of density function theory (DFT) for an anionic complex. ${ }^{8}$ The only exception occurs with the $\mathrm{Nd}-\mathrm{OH}_{2}$ coordination, where the position of the water molecule is significantly different from that observed experimentally. In the experimental structure, the water molecule is normal to the O2-O3-O4-O5 plane; the calculations, however, indicate hydrogen bonding of the water's hydrogen atoms to the carboxylate units, thereby shifting the water to two neighboring vertices of the plane and lengthening the $\mathrm{Nd}-\mathrm{OH}_{2}$ bond. While this effect may significantly influence some calculated properties such as acidity constants, the shift should not affect our thermodynamic analysis in the discussion below. 
Table 3 shows the optimized structures of the $\mathrm{Nd}^{\text {III }}$ and $\mathrm{Am}^{\text {III }}$ analogues are virtually identical, as expected given the similar ionic radii of both metal centers (i.e. $1.123 \AA$ for $\mathrm{Nd}^{\mathrm{III}}$ and $1.115 \AA$ for $A m^{\mathrm{III}}$ ). Calculated bond distances and angles of the ligand are only marginally affected by the substitution of the metal center. Variations of the $\mathrm{C}-\mathrm{N}$ and $\mathrm{C}-\mathrm{O}$ bond lengths are at most 0.001 and $0.003 \AA$, respectively. The $\mathrm{M}^{\mathrm{III}}(\mathrm{M}=\mathrm{Nd}, \mathrm{Am})$ atom is situated above the center of the square antiprism with a preference towards the hydrophilic carboxylate plane over the nitrogen atoms in the antiprism base. As with the $\mathrm{Nd}^{\mathrm{III}}$ analogue, the water molecule in the optimized

Table 3. Selected bond lengths $(\AA)$ and angles $\left({ }^{\circ}\right)$ of the complexes $\left[\mathrm{M}^{\mathrm{III}}(\mathrm{DTPA})-\mathrm{H}_{2} \mathrm{O}\right]^{2-}(\mathrm{M}=\mathrm{Nd}, \mathrm{Am})$ calculated at the DFT/B3LYP level in comparison with available experimental data (in parentheses). ${ }^{7}$

\begin{tabular}{|c|c|c|}
\hline & $\mathrm{Nd}$ & $\mathrm{Am}$ \\
\hline$R(\mathrm{M}-\mathrm{N} 1)$ & $2.854(2.751 \pm 0.005)$ & 2.893 \\
\hline$R(\mathrm{M}-\mathrm{N} 2)$ & $2.763(2.732 \pm 0.005)$ & 2.753 \\
\hline$R(\mathrm{M}-\mathrm{N} 3)$ & $2.807(2.686 \pm 0.005)$ & 2.802 \\
\hline$R(\mathrm{M}-\mathrm{O} 1)$ & $2.444(2.387 \pm 0.004)$ & 2.452 \\
\hline$R(\mathrm{M}-\mathrm{O} 2)$ & $2.469(2.454 \pm 0.004)$ & 2.444 \\
\hline$R(\mathrm{M}-\mathrm{O} 3)$ & $2.414(2.420 \pm 0.004)$ & 2.434 \\
\hline$R(\mathrm{M}-\mathrm{O} 4)$ & $2.414(2.426 \pm 0.004)$ & 2.402 \\
\hline$R(\mathrm{M}-\mathrm{O} 5)$ & $2.467(2.467 \pm 0.004)$ & 2.473 \\
\hline$R(\mathrm{M}-\mathrm{O} 6)$ & $2.704(2.449 \pm 0.004)$ & 2.698 \\
\hline$\angle \mathrm{O} 2-\mathrm{M}-\mathrm{O} 6$ & $61.9(77.2 \pm 0.2)$ & 62.4 \\
\hline$\angle \mathrm{O} 3-\mathrm{M}-\mathrm{O} 6$ & $87.3(75.2 \pm 0.2)$ & 88.0 \\
\hline$\angle \mathrm{O} 4-\mathrm{M}-\mathrm{O} 6$ & $97.6(79.5 \pm 0.2)$ & 97.5 \\
\hline$\angle \mathrm{O} 5-\mathrm{M}-\mathrm{O} 6$ & $62.0(68.3 \pm 0.2)$ & 61.7 \\
\hline
\end{tabular}

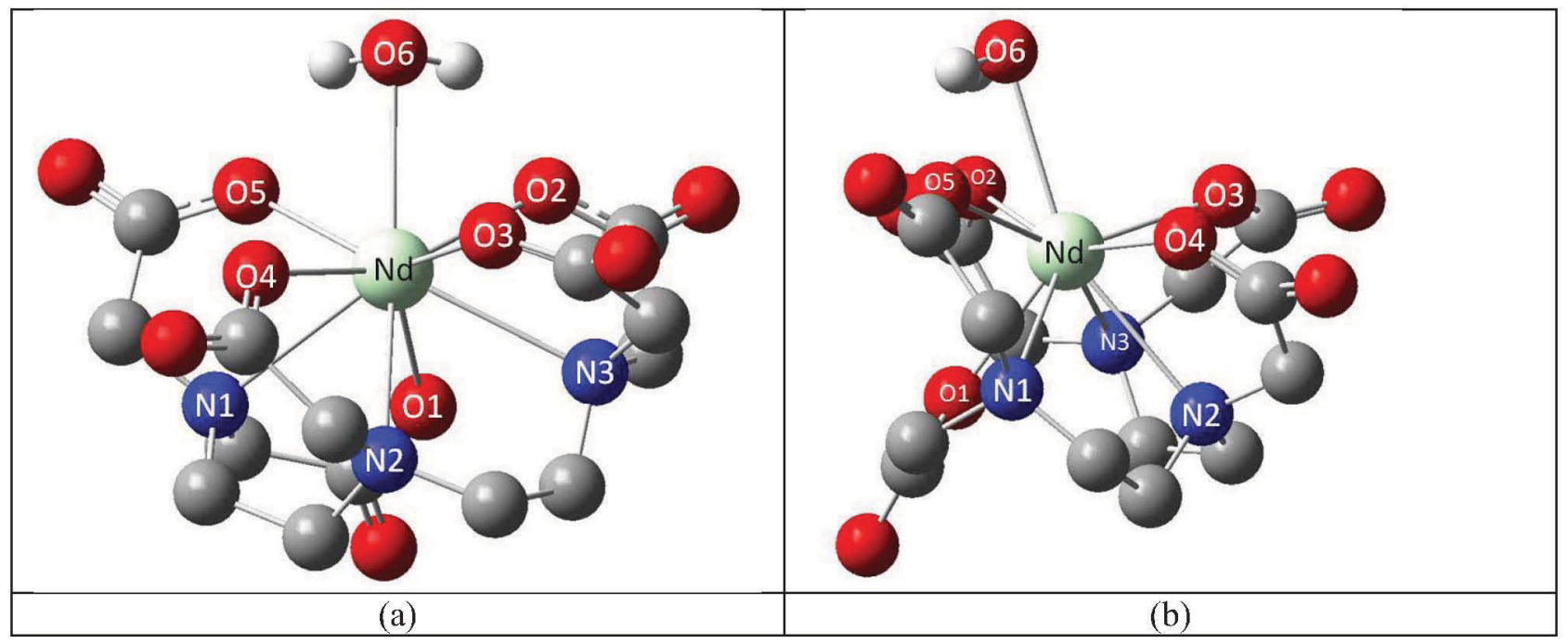

Figure 5. Front (a) and side (b) views of the optimized structure of $\left[\mathrm{Nd}^{\text {III }}(\mathrm{DTPA})\left(\mathrm{H}_{2} \mathrm{O}\right)\right]^{2-}$. Hydrogen atoms on the DTPA $^{5-}$ ligand are removed for clarity. The same atomic labels are used to describe the geometric parameters for the $\mathrm{Am}^{\mathrm{III}}$ analogue in Table 3.

$\mathrm{Am}^{\mathrm{III}}$ structure is off-center from the normal axis. Given the general similarities of these model systems, we conclude that the optimized $\left[\mathrm{Am}^{\mathrm{III}}(\mathrm{DTPA})-\mathrm{H}_{2} \mathrm{O}\right]^{2-}$ structure is an appropriate model for studying the complexation in aqueous solution. ${ }^{9,10}$ 


\section{Calculated Change in Gibbs Free Energy for the Complexation of $\left[M^{\mathrm{III}}(\mathrm{DTPA})\left(\mathrm{H}_{2} \mathrm{O}\right)\right]^{2-}(\mathrm{M}=\mathrm{Nd}, \mathrm{Am})$.}

Table 4 lists the Gibbs free energies for reaction (1) $\mathrm{M}^{\mathrm{III}}+\mathrm{H}_{5} \mathrm{DTPA}+\mathrm{H}_{2} \mathrm{O} \rightarrow\left[\mathrm{M}^{\mathrm{III}}(\mathrm{DTPA})\right.$ $\left.\mathrm{H}_{2} \mathrm{O}\right]^{2-}+5 \mathrm{H}^{+}(\mathrm{M}=\mathrm{Nd}, \mathrm{Am})$ in terms of the gas phase $\left(\Delta G_{g}^{\circ}\right)$, the aqueous solution $\left(\Delta G_{s o l v}^{\circ}\right)$, and the solvent contributions $\left(\Delta \Delta G_{s}^{\circ}\right)$. Calculations are based on the Born-Haber cycle found in Scheme 1 (see Section 8). The Gibbs free energies for the $\mathrm{Nd}^{\mathrm{III}}$ and $\mathrm{Am}^{\mathrm{III}}$ cations were evaluated with solvation shells of nine waters to simulate the aqueous environment. Similarly, the Gibbs free energies of $\mathrm{H}_{2} \mathrm{O}$ and $\mathrm{H}^{+}$(or $\mathrm{H}_{3} \mathrm{O}^{+}$) were based on a cluster model consisting of $4 \mathrm{H}_{2} \mathrm{O}$ molecules. Not only does reaction (1) represent a realistic interpretation of the chemical reaction in solution, a significant amount of error cancellation is expected when using the calculated Gibbs free energies of the solvated complexes rather than using experimental values. Also, because $\mathrm{Nd}^{\mathrm{III}}$ and $\mathrm{Am}^{\mathrm{III}}$ have similar ionic radii, solvation and entropy effects should be similar for both reactions. While these approximations affect our ability to obtain accurate absolute values for the Gibbs free energy terms, they provide a quantitative assessment of the trends found for both systems.

The calculated changes in Gibbs free energy in the gas phase for the complex [M ${ }^{\mathrm{III}}(\mathrm{DTPA})$ $\mathrm{H}_{2} \mathrm{O}^{2-}(\mathrm{M}=\mathrm{Nd}, \mathrm{Am})$ according to reaction (1) are -7.72 and $-8.34 \mathrm{kcal} \cdot \mathrm{mol}^{-1}$ for $\mathrm{Nd}$ and Am, respectively. Thus, formation of the product is spontaneous with Am being more stable than Nd by $\sim 0.5 \mathrm{kcal} \cdot \mathrm{mol}^{-1}$. These results are in agreement with experimental findings that DTPA complex is capable of successful separation of americium from lanthanides, but that the separation factor between $\mathrm{Am}$ and $\mathrm{Nd}$ is small. ${ }^{1}$ The solvation effects, $\Delta \Delta G_{s}^{\circ}$, follow the same trends as $\Delta G_{g}^{\circ}$ and favor the formation of $\left[\mathrm{Am}^{\mathrm{III}}(\mathrm{DTPA})-\mathrm{H}_{2} \mathrm{O}\right]^{2-}$ over $\left[\mathrm{Nd}^{\mathrm{III}}(\mathrm{DTPA})-\mathrm{H}_{2} \mathrm{O}\right]^{2-}$ by $\sim 0.5 \mathrm{kcal} \cdot \mathrm{mol}^{-1}$. The results indicate solvent interactions provide only a minor reduction to the free energy of complexation. Including solvation effects, complexation with Am is preferred over $\mathrm{Nd}$ by $\sim 1 \mathrm{kcal} \cdot \mathrm{mol}^{-1}$. As expected, the calculated Gibbs free energies are similar in both the gas phase and solution because of the similar ionic radii.

Table 4. Calculated change in the Gibbs free energy (kcal·mol ${ }^{-1}$ ) for the reactions $\mathrm{M}^{\mathrm{III}}+\mathrm{H}_{5} \mathrm{DTPA}+\mathrm{H}_{2} \mathrm{O} \rightarrow$ $\left[\mathrm{M}^{\mathrm{III}}(\mathrm{DTPA})-\mathrm{H}_{2} \mathrm{O}\right]^{2-}+5 \mathrm{H}^{+}(\mathrm{M}=\mathrm{Nd}, \mathrm{Am})$ in the gas phase and aqueous solution.

\begin{tabular}{|l|c|c|c|}
\hline & $\Delta G_{g}^{\circ}$ & $\Delta \Delta G_{s}^{\circ}$ & $\Delta G_{\text {solv }}^{\circ}$ \\
\hline $\mathrm{Nd}$ & -7.72 & -12.76 & -20.48 \\
\hline $\mathrm{Am}$ & -8.34 & -13.21 & -21.55 \\
\hline $\mathrm{Nd} \rightarrow \mathrm{Am}$ & -0.62 & -0.45 & -1.07 \\
\hline
\end{tabular}

It is important to point out that the predicted free energy of complexation $\left(-7.7 \sim-8.3 \mathrm{kcal} \cdot \mathrm{mol}^{-1}\right)$ is closer to weak associations (e.g., van der Walls, $\sim 2 \mathrm{kcal} \cdot \mathrm{mol}^{-1}$ ) rather than strong bonding (e.g., covalent bonds, $>17 \mathrm{kcal} \cdot \mathrm{mol}^{-1}$ ). Such small energy differences are rarely associated with dramatic changes in the molecular structure or properties. It is thought that weak covalent interactions between the "soft" Lewis base ligands such as DTPA" and the actinides are the source for the observed complexing preference over the lanthanides. ${ }^{11}$ To understand the preferred complexation with actinides, we examine the DFT results to determine the predicted nature of the interaction between the DTPA ${ }^{5-}$ ligand and the metal center.

Mulliken orbital population analysis for $\left[\mathrm{M}^{\mathrm{III}}(\mathrm{DTPA})\left(\mathrm{H}_{2} \mathrm{O}\right)\right]^{2-}(\mathrm{M}=\mathrm{Nd}, \mathrm{Am})$ shows that over the entire MOs, there is almost no additional orbital contributions to the $s, p, d$, and $f$ shells for the metal centers (Table 5), predicting that the interaction should be essentially ionic between the 
$\mathrm{M}^{\mathrm{III}}$ and DTPA ${ }^{5-}$ ligand. Upon analyzing the MOs that contain the $f$ orbitals, a clearer picture emerges as the enhanced spatial extent of the $5 f$ electron shell allows for more (albeit small) interaction with the "soft" donor groups. More importantly, the results predict a small amount of covalent interaction of the Am $5 f$ with the O $2 p$ shell, i.e., the contribution of the covalent bond to chemical bonds between nitrogen of DTPA and Am in the complex is smaller than the oxygen interaction. The contracted $4 f$ orbitals of the $\mathrm{Nd}$ analogue remain fairly contained among three MOs with little covalency with DTPA, as expected (Table 6). In the Am complex, the $f$ orbital occupations span a larger number of MOs because of its diffuse nature in comparison to the $4 f$ shell. Table 7 depicts the Mulliken orbital population of $\left[\mathrm{Am}^{\text {III }}(\mathrm{DTPA})\left(\mathrm{H}_{2} \mathrm{O}\right)\right]^{2-}$ on a per MO basis and the data show that there is weak participation in bonding occurring among the Am $5 f$ shell and the DTPA ${ }^{5-}$ ligand, manifesting as interactions between the metal center and the carboxylate chelates. Although not presented here, there is $5 f$ character $(>5 \%)$ spanning 19 different MOs and the $\mathrm{O} 2 p$ orbitals play a more pronounced role in the bonding than nitrogen. Clearly the results predict the radial extent of the $5 f$ shell is creating a small degree of covalency among the $\mathrm{Am}^{\mathrm{III}}$ and DTPA ${ }^{5-}$ ligand and this could be the driving force to explain the preferred actinide complexation over the lanthanides. However, the results are not conclusive as to the exact interaction (M-N or M-O) that allows for separation between the metal centers.

Table 5. Total Mulliken orbital populations $(s, p, d, f)$ and atomic charges $(Q)$ on the metal center for the complexes $\left[\mathrm{M}^{\mathrm{III}}(\mathrm{DTPA})\left(\mathrm{H}_{2} \mathrm{O}\right)\right]^{2-}(\mathrm{M}=\mathrm{Nd}, \mathrm{Am})$.

\begin{tabular}{|l|c|c|c|c|c|}
\hline Atom & $s$ & $p$ & $d$ & $f$ & $Q$ \\
\hline $\mathrm{Nd}$ & 0.04 & 0.01 & 0.02 & 3.00 & 2.71 \\
\hline $\mathrm{Am}$ & 0.05 & 0.02 & 0.06 & 5.95 & 2.69 \\
\hline
\end{tabular}

Table 6. Mulliken population analysis of the $\mathrm{Nd} 4 f, \mathrm{~N} 2 p$, and $\mathrm{O} 2 \mathrm{p}$ orbitals on a per MO basis for $\left[\mathrm{Nd}^{\text {III }}(\mathrm{DTPA})\left(\mathrm{H}_{2} \mathrm{O}\right)\right]^{2-}$. The $\mathrm{O} 2 p$ populations only refer to atoms bonded to the $\mathrm{Nd}$ atom.

\begin{tabular}{|l|c|c|c|c|}
\hline MO Number & Energy & $\mathrm{Nd} 4 f$ & $\mathrm{~N} 2 p$ & O 2p \\
\hline 107 & -2.65 & 0.90 & 0.01 & 0.04 \\
\hline 106 & -2.72 & 0.85 & 0.01 & 0.08 \\
\hline 105 & -2.79 & 0.82 & 0.03 & 0.07 \\
\hline
\end{tabular}

Table 7. Mulliken population analysis of the Am 5f, $\mathrm{N} 2 p$, and O $2 p$ orbitals on a per MO basis for $\left[\mathrm{Am}^{\text {III }}(\mathrm{DTPA})\left(\mathrm{H}_{2} \mathrm{O}\right)\right]^{2-}$. The $\mathrm{O} 2 p$ populations only refer to atoms bonded to the Am atom.

\begin{tabular}{|l|c|c|c|c|}
\hline MO Number & Energy & Am $5 f$ & $\mathrm{~N} 2 p$ & O 2p \\
\hline 128 -HOMO & -0.46 & 0.73 & 0.07 & 0.09 \\
\hline 127 & -0.57 & 0.77 & 0.03 & 0.11 \\
\hline 126 & -0.71 & 0.82 & 0.02 & 0.07 \\
\hline 125 & -0.76 & 0.85 & 0.01 & 0.10 \\
\hline 124 & -1.04 & 0.72 & 0.01 & 0.10 \\
\hline 118 & -1.50 & 0.53 & 0.01 & 0.05 \\
\hline
\end{tabular}


For a more complete picture, we probed the nature of the $\mathrm{M}-\mathrm{DTPA}^{5-}(\mathrm{M}=\mathrm{Nd}, \mathrm{Am})$ interaction using the atoms in the molecule (AIM) approach, which relies on an analysis of the electron density to study the bonding properties of the complexes. While the approach has only been used on a small number of actinide systems, it has been able to provide some insight into the covalent bonding nature of actinide complexes. ${ }^{12,13}$ Bond paths in AIM are defined as paths of electron density maxima in two dimensions, i.e. the electron density is higher on the path than on any neighboring line connecting the same two nuclei. In the third dimension along the optimum path, the electron density decreases away from the bonded nuclei. This minimum along the path is referred to as the bond critical point (BCP). The properties of the electron and energy densities along the BCP can be calculated in terms of the electron density, $\rho$, its Laplacian, $\nabla^{2} \rho$, the ellipticity, $\varepsilon$, and the total energy density, $H$. Covalent bonding behavior is described as values of $\rho$ greater than 0.2 and $\nabla^{2} \rho$ that are significantly less than zero. ${ }^{14}$ The total energy density at the BCP is negative for covalent interactions, and the ellipticity describes the degree of cylindrical symmetry in the bond. ${ }^{15}$

In Table 8 , the BCP data for $\left[\mathrm{M}^{\mathrm{III}}(\mathrm{DTPA})\left(\mathrm{H}_{2} \mathrm{O}\right)\right]^{2-}(\mathrm{M}=\mathrm{Nd}, \mathrm{Am})$ are presented and described in terms of the metal-ligand bonds; the metal-ligand data are averaged. Looking first at the metalnitrogen bonds, $\rho$ is a very small number, its Laplacian is positive, and the low $\varepsilon$ values indicate little deviation from cylindrical symmetry. These small values indicate very little metal-nitrogen covalency. The average energy densities, $H$, are also very small, but for $\left[\operatorname{Am}(\mathrm{DTPA})\left(\mathrm{H}_{2} \mathrm{O}\right)\right]^{2-} H$ changes sign and is more than 4 times larger in magnitude than the $\mathrm{Nd}$ analogue. These results suggest that the Am complex has slightly more covalent interactions with the nitrogen chelates, albeit small. Moving on to the metal-oxygen bonds, in particular those on DTPA ${ }^{5-}$, both molecules exhibit the same trends as seen with the nitrogen chelates, i.e. $\rho<0.2, \nabla^{2} \rho>0$, and $\varepsilon$ values are low. However, unlike the metal-nitrogen bonds, the average energy densities are negative for both complexes and the value is almost twice as large for the Am analogue. Looking specifically at $\left[\mathrm{Am}^{\mathrm{II}}(\mathrm{DTPA})\left(\mathrm{H}_{2} \mathrm{O}\right)\right]^{2-}$, the result that is surprising the average energy density is more than twice as large for the Am-O interaction than at the Am-N BCPs, suggesting that there is slightly more Am-O covalent behavior.

Table 8. Characteristics of the $\mathrm{M}-\mathrm{L}$ bond critical points $(\mathrm{BCP})$ in $\left[\mathrm{M}(\mathrm{DTPA})-\mathrm{H}_{2} \mathrm{O}\right]^{2-}(\mathrm{M}=\mathrm{Nd}, \mathrm{Am})$. The averaged values are presented for the $\mathrm{M}-\mathrm{N}$ and $\mathrm{M}-\mathrm{O}$ data.

\begin{tabular}{|l|c|c|c|}
\hline & & {$\left[\mathrm{Nd}^{\mathrm{III}}(\mathrm{DTPA})-\mathrm{H}_{2} \mathrm{O}^{2-}\right.$} & {$\left[\mathrm{Am}^{\mathrm{III}}(\mathrm{DTPA})-\mathrm{H}_{2} \mathrm{O}\right]^{2-}$} \\
\hline $\mathrm{M}-\mathrm{N}$ & $\rho$ & 0.0277 & 0.0293 \\
\hline & $\nabla^{2} \rho$ & 0.0802 & 0.0870 \\
\hline & $\varepsilon$ & 0.0871 & 0.0578 \\
\hline & $H$ & 0.000162 & -0.000734 \\
\hline $\mathrm{M}-\mathrm{O}$ & $\rho$ & 0.0504 & 0.0530 \\
\hline & $\nabla^{2} \rho$ & 0.1904 & 0.2124 \\
\hline & $\varepsilon$ & 0.0647 & 0.0648 \\
\hline & $H$ & -0.00109 & -0.00198 \\
\hline $\mathrm{M}-\mathrm{OH}_{2}$ & $\rho$ & 0.0288 & 0.0311 \\
\hline & $\nabla^{2} \rho$ & 0.0954 & 0.1037 \\
\hline & $\varepsilon$ & 0.0360 & 0.0381 \\
\hline & $H$ & 0.000156 & -0.001014 \\
\hline
\end{tabular}




\section{CONCLUSIONS}

Through the probing of plutonium-DTPA complex with a combination of electrochemical techniques and visible light spectroscopy, a better understanding of the Pu-DTPA complex has been developed. The reduction of $\mathrm{Pu}^{\mathrm{IV}}$ to $\mathrm{Pu}{ }^{\mathrm{III}}$ will alter the overall complex charge but the multiple $\mathrm{pK}_{\mathrm{A}}$ of DTPA allow for quick protonation/deprotonation. This ability of the DTPA to protonate/deprotonate makes the $\mathrm{Pu}^{\mathrm{IV} / \mathrm{III}}$ couple quasi-reversible, becoming more reversible as the sweep time increases. The Pu-DTPA complex favors the same coordination environment with $\mathrm{Pu}^{\mathrm{III}}$ and $\mathrm{Pu}^{\mathrm{IV}}$ in the $\mathrm{pH}$ range investigated here.

The chelation of $\mathrm{Pu}$ with DTPA causes a significant shift in the potential of the $\mathrm{Pu}{ }^{\mathrm{III} / \mathrm{IV}}$ couple, and the measured potential can be used to further evaluate the ratio of the $\log \beta$ of Pu-DTPA in the TALSPEAK $\mathrm{pH}$ range. The current literature values are for completely deprotonated DTPA, and might not accurately reflect the $\log \beta$ values at $\mathrm{pH} 3.5-4.0$ where there are up to four different species of DTPA present. The ratio of the $\log \beta$ for $\mathrm{Pu}^{\mathrm{IV}}$ to $\mathrm{Pu}{ }^{\mathrm{III}}$ is constant over the investigated $\mathrm{pH}$ range, supporting that one complex is the preferred complex.

The present study provides a quantitative look at the complexation and bonding of Am and $\mathrm{Nd}$ with DTPA ${ }^{5-}$. Theory is able to predict the experimental observation that Am complexation with the $\mathrm{DTPA}^{5-}$ ligand is preferred over $\mathrm{Nd}$ by $\sim 1 \mathrm{kcal} \mathrm{mol}^{-1}$. Based on the above results, preference of the DTPA ${ }^{5-}$ ligand for Am over Nd is actually a subtle combination of effects. The larger radial extent of the $5 f$ orbitals is causing increased covalency with preference for the oxygen chelates. This interpretation is supported by Mulliken population and AIM analysis. In addition, a small amount of covalent behavior between the $\mathrm{M}-\mathrm{N}$ bonds is also allowing for additional stabilization of the complex. Understanding the thermodynamic trends of these two molecules and the reasons behind their separation will aid in future experimental design.

\section{REFERENCES}

1 Weaver, B.; Kappelmann, F. A. Talkspeak: A new method of separating americium and curium from the lanthanides bu extraction from an aqueous solution of an aminopolyacetic acid complex with a monoacidic organophosphate or phosphonate; ORNL-3559; Union Carbide Corporation, Orak Ridges, TN, August 1964.

2 Nilsson, M.; Nash, K. L. Solvent Extr. Ion Exch. 2007, 25, 665.

3 Felker, L.K.; Bnder, J. L.; Benker, D. E.; Collins, E.D.; Bailey, P. D.; Bell, G. L.; Judin, R. T.; Spencer, B.B.; Del Cul, G. D.; Vedder, R. J.; Walker, E. A.; Voit, S. L. "Results, Evaluation, and Lessons Learned from the Coupled End-to-End Project Campaign 1 Using Dresden BWR Used Fuel,"ORNL/GNEP/LTR-2008-044, June 2008.

4 Bridges, N. J. Electrochemical Investigation of TALSPEAK Process SRNL-TR-2010-00259; Savannah River Nuclear Solutions, Aiken, SC, Sept. 2010.

5 Clark, D. L.; Hecker, S. S.; Jarvinen, G. D.; Neu, M. P. Plutonium. In The Chemistry of the Actinide and Transactinide Elements, Morss, L. R., Edelstein, N. M., Fuger, J., Katz, J. J., Eds.; Springer: Netherlands, 2006; Vol. 2, pp. 1113-1180.

6 Rudisill, T. S.; Kyser III, E. A. Distribution of Actinides between the Aqueous and Organic Phases in the TALSPEAK Process SRNL-STI-2010-00502, Savannah River Nuclear Solutions, Aiken, SC, Sept. 2010.

7 Petrie, S.; Stranger, R. Inorg. Chem. 2004, 43, 2597.

8 Gagliardi, L.; Cramer, C. J. Inorg. Chem. 2006, 45, 9442.

9 Cao, X.; Heidelberg, D.; Ciupka, J.; Dolg, M. Inorg. Chem. 2010, 49, 10307.

10 Petit, L.; Joubert, L.; Maldivi, P.; Adamo, C. J. Am. Chem. Soc. 2006, 128, 2190.

11 Nash, K. L. Sol. Extr. Ion Exch. 1993, 11, 729. 
12 Tassel1, M. J.; Kaltsoyannis, N. Dalton Trans. 2010, 39, 6719.

13 Matta, C. F.; Boyd, R. J. The Quantum Theory of Atoms in Molecules. Wiley-VCH: Weinheim, 2007; pp. 1-34.

14 Cremer, D.; Kraka, E. Angew. Chem. Int. Ed. 1984, 23, 627.

\section{INDICATORS OF PROJECT QUALITY AND PRODUCTIVITY}

N. J. Bridges, A. E. Visser "Understanding the Electrochemical Behavior of Actinides to aid in their Separation," Presented by A. E. Visser, before $240^{\text {th }}$ ACS National Meeting (2010), Boston, MA, Abstract I\&EC-51.

N. J. Bridges, L. E. Roy, L. R. Martin "Alternative Means of Investigation TALSPEAK Process" , Presented by N. J. Bridges, before $35^{\text {th }}$ Actinide Separation Conference (2010), Charlotte, $\mathrm{NC}$

L. E. Roy, N. J. Bridges, L. R. Martin "Theoretical Study of the Separation of Am" from $\mathrm{Nd}^{\mathrm{III}}$ with Diethylenetriaminepentaacetic Acid" in preparation 


\section{Appendix D}

\section{Thermodynamic and Kinetic Studies of the TALSPEAK Solvent Extraction Process}

Dr. Tracy Rudisill

Savannah River National Laboratory 


\section{SUMMARY}

A primary objective of DOE-NE's Nuclear Energy R\&D Roadmap is the development of sustainable nuclear fuel cycles which improve uranium resource utilization, maximize energy generation, minimize waste generation, improve safety, and complement institutional measures limiting proliferation risks.[1] Activities in progress which support this objective include the development of advanced separation technologies to recycle the actinides from used nuclear fuels. With the increased interest in the development of technology to allow closure of the nuclear fuel cycle, the TALSPEAK process is being considered for the separation of $\mathrm{Am}$ and $\mathrm{Cm}$ from the lanthanide fission products in a next generation reprocessing plant. However, at this time, the level of understanding associated with the chemistry and the control of the process variables is not acceptable for deployment of the process on an industrial scale. To address these issues, the Fuel Cycle Research and Development (R\&D) program is supporting basic scientific studies focused on developing a fundamental understanding of the thermodynamic and kinetic driving forces of the process.[2] These studies are also structured to develop the necessary competences at DOE national laboratories to investigate and understand the chemical phenomena associated with any separation process.

To better understand the stability of $\mathrm{Pu}(\mathrm{III})$ and $\mathrm{Np}(\mathrm{V})$ in the TALSPEAK process, an experimental study was performed at the Savannah River National Laboratory (SRNL) to measure the oxidation/reduction kinetics in a $1 \mathrm{M}$ buffered lactate solution containing $0.05 \mathrm{M}$ diethylenetriaminepentaacetic acid (DTPA). A small amount of $\mathrm{Pu}$ and $\mathrm{Np}$ would be present in the TALSPEAK feed due to process losses from prior recovery operations. This study builds upon our previous work which showed that $\mathrm{Pu}(\mathrm{III})$ and $\mathrm{Np}(\mathrm{V})$ are not stable in the lactic acid/DTPA solutions used as the aqueous phase in the TALSPEAK process.[3] The stability of Pu(IV)-DTPA and Np(IV)-DTPA complexes are much greater than the stability of the $\mathrm{Pu}$ (III)-DTPA and $\mathrm{Np}$ (V)-DTPA complexes, and as a result, $\mathrm{Pu}$ is slowly oxidized to $\mathrm{Pu}(\mathrm{IV})$ and $\mathrm{Np}$ is slowly reduced to $\mathrm{Np}$ (IV) due to the reduced activity of the more stable complexes.

In the present study, time-dependent ultraviolet-visible (UV-vis) spectra were used to calculate first order rate constants for the oxidation of $\mathrm{Pu}$ (III) to $\mathrm{Pu}(\mathrm{IV})$ and the reduction of $\mathrm{Np}$ (V) to $\mathrm{Np}$ (IV). The rate constants for the oxidation/reduction reactions were calculated from changes in absorbance at peaks in the spectra which are characteristic of Pu(III)-DTPA and Np(V)-DTPA complexes. The oxidation/reduction rate constants are specific to the $\mathrm{Pu}(\mathrm{III})$ and $\mathrm{Np}(\mathrm{V})$-DTPA complexes which absorb at these wavelengths. The time-dependent spectra for both elements had four to five isosbestic points which indicate the presence of multiple Pu and Np species in the lactic acid/DTPA solution. Additional experiments and analysis would be required to develop speciation diagrams that would allow the calculation of the actinide/DTPA concentrations as functions of time. The rate constants calculated for Pu resulted in halftimes for the oxidation of $\mathrm{Pu}(\mathrm{III})$ between approximately 8 and $20 \mathrm{~h}$. The half-times for the reduction of $\mathrm{Np}(\mathrm{V})$ were 17 and $171 \mathrm{~h}$. The difference in magnitude of the $\mathrm{Np}$ reduction rates was attributed to the difference in the stability of the Np(V)-DTPA complexes which absorbed at the two wavelengths of interest. A slower rate for the reduction of $\mathrm{Np}(\mathrm{V})$ than the oxidation of $\mathrm{Pu}(\mathrm{III})$ is not unexpected. The kinetics for a one electron transfer is generally faster than those where a metal oxygen bond must also be broken.

To examine the thermodynamic driving force for the extraction of actinide elements in the TALSPEAK process, an experimental study was performed to measure the distribution of $\mathrm{Np}$ (IV), $\mathrm{Pu}$ (IV), $\mathrm{Am}$ (III), and $\mathrm{Pa}(\mathrm{V})$ between the aqueous and organic phases as a function of temperature. This study extends our previous work in which distribution coefficients were measured for $\mathrm{Np}(\mathrm{V}), \mathrm{Pu}(\mathrm{III}), \mathrm{Am}(\mathrm{III})$, and $\mathrm{Pa}(\mathrm{V})$ as a function of temperature and conditional enthalpies and entropies of extraction were calculated by van't Hoff analysis.[3] In the present study, we found that $\mathrm{Am}$ (III) and especially $\mathrm{Pa}(\mathrm{V})$ were more strongly extracted into the bis-(2-ethylhexyl)phosphoric acid (HDEHP) solvent than $\mathrm{Np}$ (IV) or $\mathrm{Pu}$ (IV). The decreased extent of extraction for the 4+ actinides was attributed to the stability of the An(IV)-DTPA complexes (compared to either the $5+$ or $3+$ complexes) which limits their extraction into the HDEHP 
solvent. Conditional enthalpies and entropies of extraction for $\mathrm{Np}$ (IV) and $\mathrm{Pu}$ (IV) were calculated by van't Hoff analysis. The correlation of the distribution data for Am(III) and $\mathrm{Pa}(\mathrm{IV})$ was poor and thermodynamic properties could not be calculated. The poor correlation of the distribution data was attributed to the low concentration of HDEHP used in the experiment which resulted in an insufficient amount of activity in the organic phase to obtain consistent results. The conditional enthalpies of extraction for $\mathrm{Np}(\mathrm{IV})$ and $\mathrm{Pu}(\mathrm{IV})$ were more positive than values previously measured for $\mathrm{Np}(\mathrm{V})$ and $\mathrm{Pu}(\mathrm{III})$ which is consistent with more stable DTPA complexes limiting the extraction of $4+$ actinides.

\section{INTRODUCTION}

\section{Background}

Used reactor fuels are currently reprocessed in multiple countries using the PUREX process [4] to recover the $\mathrm{U}$ and $\mathrm{Pu}$ for future use. However, the small amounts of $\mathrm{Np}, \mathrm{Am}$, and $\mathrm{Cm}$ (minor actinides) produced during fuel irradiation are not recovered and are currently discarded with the fission products as high level waste. If desired, $\mathrm{Np}$ produced in irradiated fuels can be recovered by making slight modifications to the PUREX process to control the $\mathrm{Np}$ valence and extraction into the tributyl phosphate solvent.[5,6] For complete closure of the nuclear fuel cycle, the Am and $\mathrm{Cm}$ isotopes produced during fuel irradiation must also be recovered. The isolation and purification of the minor actinides would allow additional energy production following fabrication of fuels or targets for advanced reactors using these materials. The elimination of essentially all of the actinide materials from the high level waste generated during fuel reprocessing would also improve the performance of a geologic repository by removing radiotoxic, longlived transuranic isotopes from the waste and reducing the heat generated by radioactive decay.

An efficient separation process to recover Am and $\mathrm{Cm}$ during the reprocessing of nuclear reactor fuels has not been demonstrated on an industrial scale. One of the issues with the recovery of Am and $\mathrm{Cm}$ in a single process is the similarity of the chemistry of the lanthanide fission products. Solvent extraction processes have been developed which allow the separation of $\mathrm{Am}, \mathrm{Cm}$, and the lanthanide elements from other waste components using extractants such as malonamids (the DIAMEX process) or phosphine oxides (the TRUEX or TRPO processes).[7] The subsequent separation of the Am and Cm from the lanthanide fission products requires a separate process. In 1964, Weaver and Kappelmann [8] reported the development of a process to separate transplutonium elements from the lanthanides by preferentially extracting the lanthanides from an aqueous solution containing lactic acid and the sodium salt of DTPA into HDEHP. The process was titled TALSPEAK for Trivalent Actinide Lanthanide Separation with Phosphorous-reagent Extraction from Aqueous Komplexes. Under optimal conditions for the TALSPEAK process, $\mathrm{Nd}$ was the least extractable of the lanthanides and Cf was the most extractable of the transplutonium elements. The process can also be performed in a reverse mode in which the transplutonium elements and the lanthanide fission products are extracted into the HDEHP solvent and the transplutonium elements are stripped using a solution containing lactic acid and DTPA.

With the increased interest in the development of technology to allow closure of the nuclear fuel cycle, the TALSPEAK process is one of several options being considered for the separation of Am and Cm from the lanthanide fission products in a future generation reprocessing plant. However, at this time, the level of understanding associated with the phase equilibria and the control of the process variables is not acceptable for deployment of the process on an industrial scale. To address these issues, the Fuel Cycle $\mathrm{R} \& \mathrm{D}$ program is supporting basic scientific studies focused on developing a fundamental understanding of the thermodynamic and kinetic driving forces of the process.[2] These studies are also structured to develop the necessary competences at DOE national laboratories to investigate and understand the chemical phenomena associated with any separation process. 


\section{Objectives}

In FY10, we performed a series of experiments to measure the distribution of $\mathrm{Np}(\mathrm{V}), \mathrm{Pu}(\mathrm{III}), \mathrm{Am}(\mathrm{III})$, and $\mathrm{Pa}(\mathrm{V})$ between the aqueous and organic phases of the TALSPEAK process as a function of temperature.[3] The van't Hoff equation was then used to calculate the conditional enthalpies and entropies of extraction. During these studies, UV-vis spectroscopy was used to demonstrate that Np(V) and $\mathrm{Pu}(\mathrm{III})$ are not stable in the lactic acid/DTPA solutions used as the aqueous phase in the TALSPEAK process. The stability of $\mathrm{Np}$ (IV)-DTPA and Pu(IV)-DTPA complexes are much greater than the stability of the $\mathrm{Np}$ (V)-DTPA and Pu(III)-DTPA complexes, and as a result, $\mathrm{Np}$ is slowly reduced to Np(IV) and $\mathrm{Pu}$ is slowly oxidized to $\mathrm{Pu}(\mathrm{IV})$ due to the reduced activity of the more stable complexes.

In a continuation of our FY10 work, we have undertaken a more extensive study of the oxidation/reduction kinetics of $\mathrm{Pu}$ (III) and $\mathrm{Np}(\mathrm{V})$ in the TALSPEAK process. In this study, $\mathrm{Pu}$ (III) and $\mathrm{Np}(\mathrm{V})$ solutions were prepared in a $1 \mathrm{M}$ buffered lactate solution containing $0.05 \mathrm{M}$ DTPA. The UV-vis spectrum of the solutions was then recorded as a function of time until changes in the spectra were no longer observed. Rate constants for the reduction of $\mathrm{Np}(\mathrm{V})$ and the oxidation of $\mathrm{Pu}(\mathrm{III})$ were subsequently calculated from changes in peak absorbance. For a broader understanding of the thermodynamics of the extraction of $\mathrm{Np}$ and $\mathrm{Pu}$ in the TALSPEAK process, we also performed additional distribution coefficient measurements. In these experiments the distributions of $\mathrm{Np}$ (IV) and $\mathrm{Pu}$ (IV) between the aqueous and organic phases were measured as a function of temperature. $\mathrm{An}^{241} \mathrm{Am}$ tracer was also added to the actinide solutions which allowed us to obtain data for $\mathrm{Np}$ (IV), $\mathrm{Pu}$ (IV), $\mathrm{Am}$ (III), and $\mathrm{Pa}(\mathrm{V})$ (the decay product of $\mathrm{Np}$ ). The distribution data were used to calculate the conditional enthalpies and entropies of extraction. The experimental methods used to measure the oxidation/reduction kinetics and perform the actinide distribution coefficient measurements and discussions of the results are presented in the following sections.

\section{EXPERIMENTAL}

\section{Oxidation/Reduction Kinetics of $\mathrm{Pu}(\mathrm{III})$ and $\mathrm{Np}(\mathrm{V})$}

Actinide elements such as $\mathrm{Np}$ and $\mathrm{Pu}$ have multiple stable oxidation states in aqueous solutions; however, in previous studies, we have demonstrated that $\mathrm{Np}$ (IV) and $\mathrm{Pu}(\mathrm{IV})$ are the stable species in the aqueous phase of TALSPEAK process[3]. Qualitative experiments were performed in which UV-vis spectroscopy was used to demonstrate that $\mathrm{Np}(\mathrm{V})$ was reduced to $\mathrm{Np}$ (IV) and $\mathrm{Pu}(\mathrm{III})$ was oxidized to $\mathrm{Pu}(\mathrm{IV}$ ) over a timescale of approximately one week. In the present study, we prepared solutions containing $\mathrm{Np}(\mathrm{V})$ or $\mathrm{Pu}$ (III) in $1 \mathrm{M}$ lactic acid/ammonium lactate containing $0.05 \mathrm{M}$ DTPA at $\mathrm{pH} 3.6$ for additional spectroscopic measurements. The UV-vis spectra of the actinide solutions were recorded as a function time. The absorbances of the actinide species were then used to calculate rate constants for the oxidation/reduction kinetics. A baseline spectrum of $\mathrm{Np}$ (IV) and $\mathrm{Pu}$ (IV) solutions was also recorded to aid in the interpretation of the spectra obtained during the kinetic studies. The details of the experimental procedures are discussed in the following sections.

\section{Nuclear Materials}

The Np and Pu solutions used for the kinetic studies were previously purified by anion exchange. A characterization of each solution is provided in the Table 1 . The Pu used in the study was weapons grade (i.e., nominally $94 \%{ }^{239} \mathrm{Pu}$ and $6 \%{ }^{240} \mathrm{Pu}$ ). 
Table 1 Actinide stock solution characterizations

\begin{tabular}{cccc}
\hline \hline Element & $\begin{array}{c}\text { Activity } \\
(\mathrm{dpm} / \mathrm{mL})\end{array}$ & $\begin{array}{c}\text { Concentration } \\
(\mathrm{g} / \mathrm{L})\end{array}$ & $\begin{array}{c}\mathrm{HNO}_{3} \\
(\mathrm{M})\end{array}$ \\
\hline $\mathrm{Np}$ & $5.00 \mathrm{E}+07$ & 32.0 & 1.5 \\
$\mathrm{Pu}$ & $6.65 \mathrm{E}+09$ & 45.3 & 1.0 \\
\hline
\end{tabular}

The Np solution described in Table 1 was used in the kinetic measurements; however, the UV-vis spectrum of a $\mathrm{Np}(\mathrm{IV})$ solution in a $1 \mathrm{M}$ buffered lactate solution containing $0.05 \mathrm{M}$ DTPA at pH 3.6 was desired for reference. Since the $\mathrm{Np}$ in the stock solution was predominately in the $5+$ valence, preparation of a $\mathrm{Np}$ (IV) solution was required. The $\mathrm{Np}$ (IV) was prepared by anion exchange. The $\mathrm{Np}$ and a small quantity of ${ }^{238} \mathrm{Pu}$ in the feed solution were initially reduced with ferrous sulfamate. The concentration of ${ }^{238} \mathrm{Pu}$ was adjusted to a value which was appropriate for use as a tracer in $\mathrm{Pu}$ distribution coefficient measurements. It should be noted that the ${ }^{238} \mathrm{Pu}$ (III) readily oxidizes to ${ }^{238} \mathrm{Pu}$ (IV) (unless a holding reductant is added to the feed) and is retained by the anion exchange resin. The loaded column was washed with 7-8 $\mathrm{M}$ nitric acid to remove Fe and sulfate and was eluted using a $0.17 \mathrm{M} \mathrm{HNO}_{3}$ solution containing $0.05 \mathrm{M}$ hydrazine. The hydrazine was added to hold the $\mathrm{Np}$ in the +4 valence. The product from the column run is characterized in Table 2.

Table 2 Characterization of $\mathrm{Np}$ (IV) stock solution

\begin{tabular}{cccc}
\hline \hline Component & $\begin{array}{c}\text { Activity } \\
(\mathrm{dpm} / \mathrm{mL})\end{array}$ & $\begin{array}{c}\text { Concentration } \\
(\mathrm{g} / \mathrm{L})\end{array}$ & $\begin{array}{c}\text { Concentration } \\
(\mathrm{M})\end{array}$ \\
\hline${ }^{238} \mathrm{Pu}$ & $7.57 \mathrm{E}+07$ & 48.3 & \\
$\mathrm{HNO}_{3}$ & $6.47 \mathrm{E}+09$ & 0.17 & 1.5 \\
\hline
\end{tabular}

\section{Solution Preparation}

The Np solution used in the kinetic measurements was prepared by adding a $1-\mathrm{mL}$ aliquot of either the $\mathrm{Np}$ (IV) or $\mathrm{Np}$ (V) stock solution to $10 \mathrm{~mL}$ of a buffered lactate solution containing DTPA. When the lactic acid/ammonium lactate buffer and the DTPA were prepared, the concentrations were adjusted to account for all dilutions. The final concentrations were nominally $1 \mathrm{M}$ lactate and $0.05 \mathrm{M}$ DTPA. The $\mathrm{pH}$ of the solutions was adjusted to 3.6 by the dropwise addition of $7.8 \mathrm{M}$ ammonium hydroxide $\left(\mathrm{NH}_{4} \mathrm{OH}\right)$. The solution used to obtain the $\mathrm{Np}$ (IV) reference spectrum, was prepared using the $\mathrm{Np}$ (IV) stock solution stabilized with hydrazine (see Table 2). The Np solution used for the kinetic measurements was prepared using $\mathrm{Np}(\mathrm{V})$ (see Table 1). The preparation of the $\mathrm{Np}$ solutions including the $\mathrm{pH}$ adjustments is described in Appendix A.

Since the Pu in the stock solution (see Table 1) was predominately in the 4+ valence, an adjustment was not required prior to recording the $\mathrm{Pu}(\mathrm{IV})$ reference spectra. However, it was necessary to reduce the $\mathrm{Pu}(\mathrm{IV})$ in the stock solution to $\mathrm{Pu}$ (III) for the kinetics measurements using hydroxylamine nitrate (HAN). A $0.65-\mathrm{mL}$ aliquot of $1.77 \mathrm{M}$ HAN was combined with a $1-\mathrm{mL}$ aliquot of the Pu solution to perform the reduction. The $\mathrm{Pu}(\mathrm{III}) / \mathrm{HAN}$ solution was then combined with $9.35 \mathrm{~mL}$ of a buffered lactate solution containing DTPA. The concentrations of the lactic acid/ammonium lactate buffer and DTPA were adjusted during preparation to account for all dilutions. The final concentrations were nominally $1 \mathrm{M}$ lactate and $0.05 \mathrm{M}$ DTPA. The final concentration of HAN in the solution was approximately $0.1 \mathrm{M}$. The $\mathrm{pH}$ of the solutions was adjusted to 3.6 by the dropwise addition of $7.8 \mathrm{M} \mathrm{NH}_{4} \mathrm{OH}$. To maintain the same Pu and nitrate concentrations in all solutions, we replaced the HAN in the solution used for the 
$\mathrm{Pu}(\mathrm{IV})$ reference spectrum with an equal molar concentration of ammonium nitrate $\left(\mathrm{NH}_{4} \mathrm{NO}_{3}\right)$. The preparation of the Pu solutions including the $\mathrm{pH}$ adjustments is described in Appendix A.

\section{UV-vis Spectroscopy}

The UV-vis spectra were recorded using a Zeiss diode array spectrometer for the Pu data and an AvaSpec diode array spectrometer for the $\mathrm{Np}$ data. We used a $1 \mathrm{~cm}$ path length cuvette holder with $1 \mathrm{~cm}$ disposable cuvettes inside a radiological glovebox. The cuvette holder was connected to the spectrometer using fiber optic cables. Detailed information concerning the spectrometer components is provided below.

- Spectrometer (Pu data): Diode array spectrometer based on the Zeiss MCS module (190-1024 nm range, approximately $0.8 \mathrm{~nm} /$ pixel); interfaced to computer through Hamamatsu C4070 driver/amplifier board; power supply - Condor D.C. Power Supplies model MTLL-5W-A, computer Texas Micro industrial PC, IPC-6806P.233MHz operating Windows NT.

- Fiber optic cable: Ceramoptec or Polymicro, IR grade, 400 micron low-OH core with SMA fittings on each end.

- Data acquisition card: National Instruments AT-AI-16XE-10 Multiple I/O Board (16-bit resolution, 16 analog and 8 digital inputs).

- Light source: Ocean Optics Tungsten Halogen Lamp Housing, LS-1.

- Cuvette block: SRNL fabricated Plexiglas ${ }^{\text {TM }}$ cuvette holders with two lenses.

- Spectrometer (Np Data): Thermo-Electric Cooled Fiber Optic Spectrometer, 75 mm Avabench, 2048 pixel TE cooled and regulated CCD detector, with a $>150 \mathrm{~nm}$ Deep UV detector coating, $25 \mu \mathrm{m}$ slit size, sorting coating with 350 and $590 \mathrm{~nm}$ longpass filter for UA grating, wavelength range 200$1100 \mathrm{~nm}$ using a USB2 high speed interface to a laptop computer operating Windows XP.

Initially, the UV-vis spectrometers were set to record a $\mathrm{Pu}$ or $\mathrm{Np}$ spectrum every few minutes. As changes in the spectra became less pronounced, the recording frequency was systematically lengthened.

\section{Distribution Coefficient Measurements}

The distribution of $\mathrm{Np}$ (IV), $\mathrm{Pu}(\mathrm{IV}), \mathrm{Am}(\mathrm{III})$, and $\mathrm{Pa}(\mathrm{V})$ between $0.17 \mathrm{M}$ HDEHP (in dodecane) and an aqueous phase containing a $1 \mathrm{M}$ lactic acid/ammonium lactate buffer and $0.05 \mathrm{M}$ DTPA was measured as a function of temperature. The experimental methods used to perform this work are described below.

\section{Nuclear Materials}

The actinide distribution coefficient measurements were made using dilute solutions of the nuclear materials. To achieve good counting statistics, the desired range of activities for the $\mathrm{Np}, \mathrm{Pu}$, and $\mathrm{Am}$ were $10^{5}-10^{6}, 10^{8}-10^{9}$, and $10^{5}-10^{6} \mathrm{dpm} / \mathrm{mL}$, respectively. The Pa distribution coefficients were measured using the activity $\left(10^{5}-10^{6} \mathrm{dpm} / \mathrm{mL}\right)$ which was in secular equilibrium with the ${ }^{237} \mathrm{~Np}$.

The Np(IV) stock solution prepared for the UV-vis spectroscopy (see Table 2) was used to perform the distribution coefficient measurements. However, the $0.05 \mathrm{M}$ hydrazine used to elute the $\mathrm{Np}$ (IV) from the anion exchange resin did not prevent the oxidation of the $\mathrm{Np}(\mathrm{IV})$ to $\mathrm{Np}(\mathrm{V})$ over several months. A UV-vis spectrum of the solution recorded prior to use showed that $\mathrm{Np}(\mathrm{V})$ was the predominate valence in the solution. Rather than refreshing the $\mathrm{Np}$ (IV) by anion exchange, we chose to add the $\mathrm{Np}$ to the buffered lactate solution containing DTPA and allow the reduction to occur naturally over 2-3 weeks. The stability of the Np(IV)-DTPA complex results in the slow reduction of $N p(V)$ to $N p(I V)$. [3] The

${ }^{238} \mathrm{Pu}$ present in the solution was used as a tracer for measurement of the distribution of $\mathrm{Pu}$ between the HDEHP and the buffered lactate solution containing DTPA. 
The ${ }^{241} \mathrm{Am}$ used for the distribution coefficient measurements was initially purified using a chromatographic resin. A stock solution was prepared by diluting a $0.1 \mathrm{~mL}$ aliquot of a $1.30 \times 10^{9} \mathrm{dpm} / \mathrm{mL}$ solution with $10 \mathrm{~mL}$ of $0.1 \mathrm{M} \mathrm{HNO}_{3}$. The final Am and $\mathrm{HNO}_{3}$ concentrations were $1.28 \times 10^{7} \mathrm{dmp} / \mathrm{mL}\left(1.7 \times 10^{-3} \mathrm{~g} / \mathrm{L}\right)$ and $0.11 \mathrm{M}$, respectively.

\section{Solvent Preparation}

The organic phase used for all distribution coefficient measurements was 0.17 M HDEHP. This concentration was used to support collaborative work at the Idaho National Laboratory where the same concentration is currently being used.[2] The desired concentration of HDEHP was prepared by diluting with dodecane. To prepare the solution, the target mass of HDEHP was transferred to a volumetric flask which was diluted to volume with the diluent.

\section{Extraction Experiments}

The aqueous phase used for the actinide distribution coefficient measurements was prepared by initially adding a $1-\mathrm{mL}$ aliquot of the $48.3 \mathrm{~g} / \mathrm{L} \mathrm{Np}$ stock solution (see Table 2) to $11.4 \mathrm{~mL}$ of a buffered lactate solution containing DTPA. The Np solution contained $0.17 \mathrm{~g} / \mathrm{L}{ }^{238} \mathrm{Pu}$ which was used as tracer to measure the $\mathrm{Pu}(\mathrm{IV})$ distribution coefficients. Once the solutions were combined, the distribution coefficient measurements were deferred for 18 days to allow the natural reduction of the $\mathrm{Np}(\mathrm{V})$ to $\mathrm{Np}$ (IV) which was desired for the distribution coefficient measurements. Previous valence adjustment studies [3] and the kinetics experiments performed as part of this work showed that 18 days is a sufficient time for essentially complete reduction of the $\mathrm{Np}(\mathrm{V})$ to $\mathrm{Np}(\mathrm{IV})$. Following valence adjustment, a $250-\mu \mathrm{L}$ aliquot of the Am stock solution was added to the lactate solution containing the $\mathrm{Np}$ and $\mathrm{Pu}$. All transfers of solution were performed using calibrated pipettes with precisions and accuracies typically less than $1 \%$. Following the addition of the Am tracer, the $\mathrm{pH}$ of the solution was adjusted to 3.6 by the dropwise addition of $3.9 \mathrm{M} \mathrm{NH}_{4} \mathrm{OH}$. The $\mathrm{pH}$ of the solution was measured using an Accumet ${ }^{\circledR}$ Basic $\mathrm{AB} 15 \mathrm{pH}$

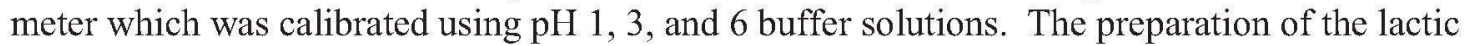
acid/DTPA solution and calculations which summarize the final composition of the aqueous phase used in the TALSPEAK extraction experiments are presented in Appendix B.

The actinide distribution coefficients were measured as a function of temperature. The temperatures were selected to bracket the potential operating range. The conditions for each series of measurements are summarized in Table 3.

Table 3 Temperatures for TALSPEAK extraction experiments

\begin{tabular}{cc}
\hline \hline Experiment & $\begin{array}{c}\text { Temperature } \\
\left({ }^{\circ} \mathrm{C}\right)\end{array}$ \\
\hline TAL-7 & 39 \\
TAL-8 & 15 \\
TAL-9 & 27 \\
\hline
\end{tabular}

To perform a series of extractions, $2 \mathrm{~mL}$ of the aqueous phase (containing the actinide elements in the $1 \mathrm{M}$ lactic acid/ammonium lactate buffer and $0.05 \mathrm{M}$ DTPA) were combined with $2 \mathrm{~mL}$ of the HDEHP solvent. A 1:1 organic to aqueous ratio was used for all extractions. Two milliliters of the aqueous and organic phases were added to six, $15 \mathrm{~mL}$ centrifuge tubes. The centrifuge tubes were capped and placed in an Eppendorf Thermomixer R heating/cooling block to maintain the extraction temperature at the desired value. The heating/cooling block also provided agitation at a maximum of $750 \mathrm{RPM}$. Once the extraction temperature was reached, the aqueous and organic phases were mixed for $1 \mathrm{~h}$. Since the intensity of the mixing was not sufficient to form an emulsion at the conical end of the centrifuge tubes, each tube was removed from heating/cooling block and manual mixed by shaking the tube. Manual 
mixing was performed at 15,30 , and 45 min into the extraction period. Once the extraction period was complete, the phases were allowed to equilibrate at temperature for $1 \mathrm{~h}$. At the beginning of the equilibration period, a cap was removed from one of the centrifuge tubes to allow insertion of a calibrated thermometer. The extraction temperatures given in Table 3 were recorded using this thermometer.

To ensure that the extraction temperature remained constant during sampling, the organic and aqueous phases were sampled using a disposable transfer pipette without removing the centrifuge tube from the heating/cooling block. The procedure involved premarking pipettes at a height which placed the tip at the middle of the organic phase when the mark was even with the top of the centrifuge tube. This procedure allowed the removal of an approximate $1 \mathrm{~mL}$ sample of the organic phase. To remove a sample of the aqueous phase, the pipette bulb was compressed and inserted into the centrifuge tube until the tip touched the bottom. An approximate $1 \mathrm{~mL}$ sample of the aqueous phase was then removed from the bottom of the tube by releasing the bulb. The ratios of the $\mathrm{Np}, \mathrm{Pu}, \mathrm{Am}$, and $\mathrm{Pa}$ activities in the aqueous and organic phases were measured by gamma spectroscopy.

\section{RESULTS AND DISCUSSION}

\section{Oxidation/Reduction Kinetics of $\mathrm{Pu}(\mathrm{III})$ and $\mathrm{Np}(\mathrm{V})$}

\section{$\mathrm{Pu}(\mathrm{III})$ Oxidation Kinetics}

During the Pu(III) oxidation experiments, the UV-vis spectrum of the buffered lactate solutions containing DTPA were recorded as a function of time. A reference spectrum was also obtained for $\mathrm{Pu}(\mathrm{IV})$ in the same solution matrix for use in interrupting the time-dependent spectra which were recorded. To illustrate the changes which occurred in the spectrum, the $\mathrm{Pu}(\mathrm{IV})$ reference spectrum and the initial spectrum recorded during each oxidation experiment were plotted on Figures 1 and 2. The $\mathrm{Pu}$ (III) oxidation experiments differed in the time which elapsed prior to recording the initial spectrum after combining the $\mathrm{Pu}(\mathrm{III})$ and the lactic acid/DTPA solutions due to the sequence in which the $\mathrm{pH}$ was adjusted and the solutions combined. The composition of both solutions was the same. The initial spectrum from Experiment 1 was not recorded until approximately $30 \mathrm{~min}$ after the solutions were combined. In this experiment, the $\mathrm{pH}$ was adjusted to 3.6 following the addition of the $\mathrm{Pu}$ (III) solution to the buffered lactate solution. In the second experiment, the initial spectrum was recorded within 2-3 min after combining the solutions. To decrease the time required to obtain the initial spectrum, we added the same volume of $\mathrm{NH}_{4} \mathrm{OH}$ used to adjust the $\mathrm{pH}$ of the solution used in Experiment 1 to the lactic acid/DTPA solution used in Experiment 2 prior to adding the Pu(III) solution. The initial Pu(III) spectra on both Figures 1 and 2 show the presence of Pu(IV); however, the peaks attributed to the presence of $\mathrm{Pu}(\mathrm{IV})$ at 448 and $499 \mathrm{~nm}$ are more pronounced for Experiment 1 (Figure 1) which is indicative of a higher $\mathrm{Pu}(\mathrm{IV})$ concentration in the solution.

To investigate the rate of oxidation of $\mathrm{Pu}$ (III) to $\mathrm{Pu}$ (IV) in a buffered lactate solution containing DTPA, we used the change in absorbance at 448 and $499 \mathrm{~nm}$ (i.e., $\mathrm{Pu}(\mathrm{IV})$ ) and at two wavelengths attributed to the presence of $\mathrm{Pu}$ (III) (574 and $595 \mathrm{~nm}$ ). In Experiment 1, spectra were recorded for $164 \mathrm{~h}$; however, little change in the spectra was observed after approximately $50 \mathrm{~h}$. Therefore, spectra were only recorded for approximately $45 \mathrm{~h}$ during Experiment 2. Figure 3 shows a plot of absorbance versus time for each wavelength of interest. The absorbances at 448 and $499 \mathrm{~nm}$ are proportional to the concentration of $\mathrm{Pu}(\mathrm{IV})$-DTPA species in the solution for constant molar absorptivities. The data from both experiments show a steady rise until approximately $25 \mathrm{~h}$. Slightly beyond this point in time the absorbances actually peak and show both upward and downward variations. The change in the data can likely be attributed to deviations in Beer's law where the absorbance of the Pu(IV)-DTPA species at these wavelengths is no longer proportional to concentration due to changes in the molar absorptivity. Shifts in the spectra baseline over time may have also caused some of the variability in the data. The absorbances at 574 and $595 \mathrm{~nm}$ are proportional to the concentration of $\mathrm{Pu}(\mathrm{III})$-DTPA species in the solution and steadily 
decrease as the $\mathrm{Pu}(\mathrm{III})$ is oxidized to $\mathrm{Pu}(\mathrm{IV})$. It is unclear why the absorbance data from Experiment 1 show a slight increase at approximately $25 \mathrm{~h}$ unless the variability is due to temporary shift in the spectra baseline.

It is noteworthy that the oxidation of $\mathrm{Pu}$ (III) proceeded at a faster rate in Experiment 2 than it did in Experiment 1 (Figure 3). We hypothesize that the difference in rate was due to the difference in $\mathrm{pH}$ when the Pu(III) was combined with the lactic acid and DTPA. In Experiment 1, the buffered lactate solution containing the DTPA was initially at a $\mathrm{pH}$ of 3.6. Adding the $\mathrm{Pu}$ (III) solution reduced the $\mathrm{pH}$ to a value of 3.3. The $\mathrm{pH}$ was subsequently adjusted back to a value of 3.6 by the addition of $\mathrm{NH}_{4} \mathrm{OH}$. The addition of an equal volume of $\mathrm{NH}_{4} \mathrm{OH}$ prior to adding the $\mathrm{Pu}$ (III) to the lactic acid/DTPA solution in Experiment 2 resulted in a $\mathrm{pH}$ greater than 3.6. The higher $\mathrm{pH}$ would increase the extent of dissociation of the DTPA (and the lactic acid) which would likely have an effect on the initial Pu(III)-DTPA complexation. The complexes which initially formed in Experiment 2 appear to have facilitated a more rapid oxidation of the $\mathrm{Pu}$ (III) to Pu(IV). It should also be noted that the final absorbance at both 448 and $499 \mathrm{~nm}$ was approximately the same for both experiments; therefore, it appears that the Pu(IV)-DTPA species associated with each absorbance was likely the same.

The data in Figure 3 for absorbances at 574 and $595 \mathrm{~nm}$ were used to calculate Pu(III) oxidation rates by assuming the rate of reaction depends only on the concentration of $\mathrm{Pu}$ (III) (i.e., first-order reaction). The first order rate law is given by equation (1),

$$
-\frac{\mathrm{dC}_{\mathrm{Pu}(\mathrm{III})}}{\mathrm{dt}}=\mathrm{kC}_{\mathrm{Pu}(\mathrm{II})}
$$

where $\mathrm{C}_{\mathrm{Pu}(\mathrm{III})}$ is the concentration of $\mathrm{Pu}$ (III) and $\mathrm{k}$ is the rate constant. If the initial concentration of $\mathrm{Pu}$ (III) is $\mathrm{C}_{0 \mathrm{Pu}(I I)}$, then the concentration of $\mathrm{Pu}(\mathrm{III})$ at time $\mathrm{t}$ is given by equation (2).

$$
\ln \left(\frac{\mathrm{C}_{\mathrm{Pu}(\mathrm{III})}}{\mathrm{C}_{0 \mathrm{Pu}(\mathrm{III})}}\right)=-\mathrm{kt}
$$

Inspection of equation (2) shows that a plot of $\ln \left(\mathrm{C}_{\mathrm{Pu}(\mathrm{III})} / \mathrm{C}_{\mathrm{OPu}(\mathrm{III})}\right)$ versus time ( $\mathrm{t}$ ) should be linear with a slope of - $\mathrm{k}$. To calculate the concentration of Pu(III) (from Beer's law), the molar absorptivity of the $\mathrm{Pu}$ (III)-DTPA complexes at the two wavelengths must be known. However, the ratio of concentrations in equation (2) can be replaced by the ratio of absorbances measured in Experiments 1 and 2 assuming the molar absorptivity remains constant (equation (3)).

$$
\ln \left(\frac{A_{\text {Pu(III) }}}{A_{0 \text { Pu(III) }}}\right)=-k t
$$

In equation (3), $\mathrm{A}_{\mathrm{Pu}(\mathrm{III})}$ is the absorption of the $\mathrm{Pu}$ (III)-DTPA complex at time $t$ and $\mathrm{A}_{0 \mathrm{Pu}(\mathrm{III})}$ is the initial absorbance at time zero. The rate constants calculated in this manner are specific to the Pu(III)-DTPA complexes which absorb at 574 and $595 \mathrm{~nm}$. The time-dependent spectra recorded in both experiments show at least four and perhaps five isosbestic points (Figures 4 and 5). An isosbestic point is a wavelength where the molar absorptivity is the same for two materials that are interconvertible without regard to the equilibrium position of the reaction between them.[9] The presence of isosbestic points indicates that multiple Pu species are present in the lactic acid/DTPA solution; therefore, additional experiments and analysis would be required to develop a speciation diagram that would allow the calculation of the $\mathrm{Pu}(\mathrm{III} / \mathrm{IV})$-DTPA concentrations as a function of time.

First order rate constants for the oxidation of $\mathrm{Pu}(\mathrm{III})$ to $\mathrm{Pu}(\mathrm{IV})$ were calculated from the absorbance data on Figure 3. The absorbance measured in the first spectrum at each wavelength was used as the initial absorbance. Plots of $\ln \left(\mathrm{A} / \mathrm{A}_{0}\right)$ versus time are shown in Figures 6 and 7, for Experiments 1 and 2, 
respectively. The first order rate constants were calculated by linear regression. The data which tails away from the linear region of the curves were not used in the analysis to calculate the rate constants. The rate constants for the $\mathrm{Pu}$ (III) oxidation are given in Table 4. The calculations used to obtain the rate constants are summarized in Appendix C.

Table 4 First order rate constants for the oxidation of $\mathrm{Pu}(\mathrm{III})$

\begin{tabular}{ccccccc}
\hline \hline Experiment & $\mathrm{k}$ & $\begin{array}{c}574 \mathrm{~nm} \\
\text { Standard } \\
\text { Deviation }\end{array}$ & $\begin{array}{c}\text { Relative } \\
\text { Standard } \\
\text { Deviation }\end{array}$ & $\mathrm{k}$ & $\begin{array}{c}595 \mathrm{~nm} \\
\text { Standard } \\
\text { Deviation }\end{array}$ & $\begin{array}{c}\text { Relative } \\
\text { Standard } \\
\text { Deviation } \\
(\%)\end{array}$ \\
\hline 1 & $\left(\mathrm{~h}^{-1}\right)$ & $\left(\mathrm{h}^{-1}\right)$ & $\left(\mathrm{h}^{-1}\right)$ & $\left(\mathrm{h}^{-1}\right)$ & $\begin{array}{c}\text { (\%) } \\
2\end{array}$ \\
\hline & 0.0350 & 0.0003 & 0.86 & 0.0588 & 0.0006 & 1.0 \\
\hline
\end{tabular}

A comparison of the rate constants for Experiments 1 and 2 shows that the $\mathrm{Pu}(\mathrm{III})$ was more rapidly oxidized in Experiment 2. We attributed the difference in the oxidation rate (and the rate constants) to the difference in $\mathrm{pH}$ when the $\mathrm{Pu}$ (III) was combined with the lactic acid and DTPA. The rate constants measured using the data at $574 \mathrm{~nm}$ are about $60 \%$ of the values which were measured using the data at $595 \mathrm{~nm}$. The difference in the magnitude of the rate constants may be attributed to the oxidation of different Pu(III)-DTPA species (with different stabilities) which absorb at 574 and $595 \mathrm{~nm}$. The rate constants calculated at $574 \mathrm{~nm}$ are also biased low. To calculate the rate constants, we assumed that the absorbances at 574 and $595 \mathrm{~nm}$ returned to zero (i.e., the baseline) upon complete oxidation of the Pu(III). This assumption is not entirely correct for the absorbance at $574 \mathrm{~nm}$. A small peak between 574 and $575 \mathrm{~nm}$ is present in the $\mathrm{Pu}(\mathrm{IV})$ reference spectrum (Figures 1 and 2). A nonzero baseline artificially reduces the rate constant; therefore, the constant calculated from the data at $574 \mathrm{~nm}$ is conservatively low. The times required for one-half of the Pu(III) to oxidize to $\mathrm{Pu}(\mathrm{IV})$ based on the rate constants calculated from the absorption data are given in Table 5.

Table 5 Half-time for the oxidation of $\mathrm{Pu}(\mathrm{III})$ to $\mathrm{Pu}(\mathrm{IV})$

\begin{tabular}{ccc}
\hline \hline Experiment & $\begin{array}{c}\text { Half-time } \\
\text { at 574 nm } \\
\text { (h) }\end{array}$ & $\begin{array}{c}\text { Half-time } \\
\text { at 595 nm } \\
\text { (h) }\end{array}$ \\
\hline 1 & 19.8 & 11.8 \\
2 & 13.9 & 7.8 \\
\hline
\end{tabular}

\section{Np(V) Reduction Kinetics}

Time-dependent UV-vis spectra were recorded to evaluate the reduction kinetics of $N p(V)$ in a buffered lactate solution containing DTPA. A reference spectrum was also obtained for $\mathrm{Np}(\mathrm{IV})$ in the same solution matrix for use in interrupting the spectra which were recorded as a function of time. To illustrate the changes which occurred in the spectrum, the $\mathrm{Np}$ (IV) reference spectrum and the initial (i.e., $1 \mathrm{~h}$ ) and final (i.e., $330 \mathrm{~h}$ ) spectra recorded during the kinetics experiment are plotted on Figure 8. Due to the time required to adjust the $\mathrm{pH}$ of the $\mathrm{Np}(\mathrm{V})$ solution, the first spectrum was not recorded until approximately one hour following the combination of the $\mathrm{Np}(\mathrm{V})$ and the lactic acid/DTPA solutions. Inspection of the initial $\mathrm{Np}(\mathrm{V})$ spectrum shows the presence of $\mathrm{Np}(\mathrm{IV})$. A small peak at $739 \mathrm{~nm}$ (which is attributed to the presence of $\mathrm{Np}(\mathrm{IV})$ ) is already observed. Comparison of the $\mathrm{Np}(\mathrm{IV})$ reference spectrum and the final $\mathrm{Np}(\mathrm{V})$ spectrum shows the features of the two spectra are generally the same.

To evaluate the rate of reduction of $\mathrm{Np}(\mathrm{V})$ to $\mathrm{Np}(\mathrm{IV})$ in a buffered lactate solution containing DTPA, we used the change in absorbance at 739 and $866 \mathrm{~nm}$ (attributed to $\mathrm{Np}$ (IV)) and at two wavelengths 
attributed to the presence of $\mathrm{Np}(\mathrm{V})(618$ and $1029 \mathrm{~nm})$. Spectra were recorded for $330 \mathrm{~h}$; however, little change in the spectra were observed after approximately $200 \mathrm{~h}$. Figure 9 shows a plot of absorbance versus time for each wavelength of interest for this time period. The absorbances at 739 and $866 \mathrm{~nm}$ are proportional to the concentration of $\mathrm{Np}$ (IV)-DTPA species in the solution for constant molar absorptivities. However, the absorbance measured at $739 \mathrm{~nm}$ as time approaches $200 \mathrm{~h}$ is probably beyond the linear range due to the high concentration of $\mathrm{Np}$ (IV). The absorbances at 618 and $1029 \mathrm{~nm}$ are proportional to the concentration of $\mathrm{Np}(\mathrm{V})$-DTPA species and steadily decrease over time as the $\mathrm{Np}(\mathrm{V})$ is reduced to $\mathrm{Np}(\mathrm{IV})$.

The data in Figure 9 for absorbances at 618 and $1029 \mathrm{~nm}$ were used to calculate the $\mathrm{Np}(\mathrm{V})$ reduction rate constants by assuming the rate of reaction is first order. An equation analogous to equation (1) can be solved for the concentration of $\mathrm{Np}(\mathrm{V})$ as a function of time $\left(\mathrm{C}_{\mathrm{Np}(\mathrm{V})}\right)$ given the initial concentration of $\mathrm{Np}(\mathrm{V})\left(\mathrm{C}_{0 \mathrm{~Np}(\mathrm{~V})}\right)$ and the first order rate constant $(\mathrm{k})$. As shown by equation (2), a plot of $\ln \left(\mathrm{C}_{\mathrm{Np}(\mathrm{v})} / \mathrm{C}_{0 \mathrm{~Np}(\mathrm{~V})}\right)$ versus time $(t)$ should be linear with a slope of $-k$. Since the molar absorptivities for the Np(V)-DTPA species at 618 and $1029 \mathrm{~nm}$ are not known and concentrations cannot be calculated, the ratios of absorbances (see equation (3)) were used to calculate the rate constants for the $\mathrm{Np}(\mathrm{V})$ reduction. The rate constants calculated in this manner are specific to the $\mathrm{Np}(\mathrm{V})$-DTPA complexes which absorb at 618 and $1029 \mathrm{~nm}$. The time-dependent spectra for the $\mathrm{Np}(\mathrm{V})$ reduction show at least five isosbestic points (Figure 10), which indicates that multiple Np species are present in the lactic acid/DTPA solution. Additional experiments and analysis would be required to develop a speciation diagram that would allow the calculation of the $\mathrm{Np}(\mathrm{V} / \mathrm{IV})$-DTPA concentrations as a function of time.

First order rate constants for the reduction of $\mathrm{Np}(\mathrm{V})$ were calculated from the absorbance data on Figure 9. Since the absorbances at 618 and $1029 \mathrm{~nm}$ are not rapidly changing in the first several spectra, the absorbance measured in the first spectrum at each wavelength was used as the initial absorbance. Plots of $\ln \left(\mathrm{A} / \mathrm{A}_{0}\right)$ versus time are shown in Figure 11. The first order rate constants were calculated by linear regression. Since the 618 and $1029 \mathrm{~nm}$ peaks in the spectra were so small, the signal to noise ratio became an issue for both calculations. Changes in the spectra baseline also resulted in absorbances which were less than zero. The regression analysis for the absorption data at $618 \mathrm{~nm}$ only includes data up to approximately $160 \mathrm{~h}$. Only data up to approximately $60 \mathrm{~h}$ were used to calculate the rate constant for the reduction of the $\mathrm{Np}(\mathrm{V})$-DTPA species which absorbed at $1029 \mathrm{~nm}$. The rate constants for the $N p(\mathrm{~V})$ reduction are given in Table 6 . The calculations used to obtain the rate constants are summarized in Appendix C.

Table 6 First order rate constants for the reduction of $\mathrm{Np}(\mathrm{V})$

\begin{tabular}{|c|c|c|c|c|c|}
\hline \multicolumn{3}{|c|}{$618 \mathrm{~nm}$} & \multicolumn{3}{|c|}{$1029 \mathrm{~nm}$} \\
\hline $\mathrm{k}$ & Standard & Relative & $\mathrm{k}$ & Standard & Relative \\
\hline & Deviation & Standard & & Deviation & Standard \\
\hline$\left(\mathrm{h}^{-1}\right)$ & $\left(\mathrm{h}^{-1}\right)$ & $\begin{array}{c}\text { Deviation } \\
(\%)\end{array}$ & $\left(h^{-1}\right)$ & $\left(h^{-1}\right)$ & $\begin{array}{c}\text { Deviation } \\
(\%)\end{array}$ \\
\hline 0.00406 & 0.00014 & 3.4 & 0.0409 & 0.0007 & 1.7 \\
\hline
\end{tabular}

The rate constant measured using the data at $618 \mathrm{~nm}$ is an order of magnitude less than the value measured at $1029 \mathrm{~nm}$. The difference in the magnitude of the rate constants (and the reduction rate) can likely be attributed to the Np(V)-DTPA speciation. The species which absorbs at $618 \mathrm{~nm}$ likely has a greater stability than the species which absorbs at $1029 \mathrm{~nm}$. The times required for one-half of the $\mathrm{Np}(\mathrm{V})$ to reduce to $\mathrm{Np}$ (IV) based on the rate constants are given in Table 7 . 
Table 7 Half-time for the reduction of $\mathrm{Np}(\mathrm{V})$ to $\mathrm{Np}(\mathrm{IV})$

\begin{tabular}{cc}
\hline \hline $\begin{array}{c}\text { Half-time } \\
\text { at } 618 \mathrm{~nm} \\
(\mathrm{~h})\end{array}$ & $\begin{array}{c}\text { Half-time } \\
\text { at } 1029 \mathrm{~nm}\end{array}$ \\
\hline 171 & (h) \\
\hline
\end{tabular}

\section{Distribution Coefficient Measurements}

\section{Actinide Distribution during TALSPEAK Extractions}

The distribution coefficient for the actinides in the TALSPEAK process is defined as the ratio of the activity of the actinide element in the organic phase $\left(A_{A n, o}\right)$ to the activity of the actinide element in the aqueous phase $\left(\mathrm{An}_{\mathrm{An}, \mathrm{a}}\right)$ (equation 4$)$.

$$
\mathrm{D}_{\mathrm{An}, \mathrm{o} / \mathrm{a}}=\frac{\mathrm{A}_{\mathrm{An}, \mathrm{o}}}{\mathrm{A}_{\mathrm{An}, \mathrm{a}}}
$$

Based on this definition, the distribution coefficients for $\mathrm{Np}, \mathrm{Pu}, \mathrm{Am}$, and $\mathrm{Pa}$ in the TALSPEAK extraction experiments were calculated from the ratio of the total number of counts in the organic phase compared to the aqueous phase. The actual activity of each actinide element in each phase was not calculated to eliminate the uncertainty associated with calibrating the detector for a specific geometry. The actinide distribution coefficients for the triplicate extraction experiments at each temperature are given in Appendix D. The average values and relative standard deviations are shown in Tables 8 for each element.

Table 8 Actinide distribution coefficients for TALSPEAK extractions

\begin{tabular}{ccccccc}
\hline \hline Element & \multicolumn{2}{c}{$15{ }^{\circ} \mathrm{C}$} & \multicolumn{2}{c}{$27{ }^{\circ} \mathrm{C}$} & \multicolumn{2}{c}{$39{ }^{\circ} \mathrm{C}$} \\
& $\mathrm{D}_{\text {An, o/a }}$ & $\begin{array}{c}\text { Relative } \\
\text { Std. Dev. } \\
(\%)\end{array}$ & $\mathrm{D}_{\text {An, o/a }}$ & $\begin{array}{c}\text { Relative } \\
\text { Std. Dev. } \\
(\%)\end{array}$ & $\mathrm{D}_{\text {An, o/a }}$ & $\begin{array}{c}\text { Relative } \\
\text { Std. Dev. } \\
(\%)\end{array}$ \\
\hline $\mathrm{Np}(\mathrm{IV})$ & $9.79 \mathrm{E}-03$ & 12.6 & $7.76 \mathrm{E}-03$ & 6.4 & $6.61 \mathrm{E}-03$ & 20.2 \\
$\mathrm{Pu}(\mathrm{IV})$ & $2.60 \mathrm{E}-02$ & 5.8 & $2.73 \mathrm{E}-02$ & 4.9 & $2.71 \mathrm{E}-02$ & 0.0 \\
$\mathrm{Am}(\mathrm{III})$ & $5.05 \mathrm{E}-02$ & 5.2 & $5.11 \mathrm{E}-02$ & 6.2 & $4.76 \mathrm{E}-02$ & 15.1 \\
$\mathrm{~Pa}(\mathrm{~V})$ & $9.55 \mathrm{E}+00$ & 10.7 & $1.03 \mathrm{E}+01$ & 4.4 & $9.54 \mathrm{E}+00$ & 12.9 \\
\hline
\end{tabular}

Inspection of the data in Table 8 shows that $\mathrm{Am}(\mathrm{III})$ and especially $\mathrm{Pa}(\mathrm{V})$ were more strongly extracted into the HDEHP solvent than $\mathrm{Np}$ (IV) and $\mathrm{Pu}$ (IV). The decreased extent of extraction for the 4+ actinides can be attributed to the stability of the An(IV)-DTPA complexes (compared to either the $5+$ or $3+$ complexes) which limits their extraction into the HDEHP solvent. The equilibrium constants $(\log \mathrm{K})$ for the $\mathrm{Np}$ (IV)-DTPA complex in 0.5 and $1.0 \mathrm{M} \mathrm{NaClO}_{4}$ are $29.29 \pm 0.02$ and $30.33 \pm 0.12$, respectively.[10] The equilibrium constant for the $\mathrm{Np}(\mathrm{V})$-DTPA complex is not reported; however, equilibrium constants for both the $\mathrm{Np}$ (IV) and $\mathrm{Np}(\mathrm{V})$-ethylenediaminetetraacetic acid (EDTA) complexes are available. The ( $\log \mathrm{K}$ ) value for the $\mathrm{Np}(\mathrm{IV})$ complex in $1.0 \mathrm{M} \mathrm{NaClO}_{4}$ is $24.55 \pm 0.03$ while the $(\log \mathrm{K}$ ) value for the $\mathrm{Np}$ (V) complex in $0.1 \mathrm{M} \mathrm{NaClO}_{4}$ is only $7.33 \pm 0.06$.[10] By analogy, the equilibrium constant for the $\mathrm{Np}$ (IV)-DTPA complex should also be orders of magnitude larger than the equilibrium constant for the $\mathrm{Np}(\mathrm{V})$-DTPA complex. The Pu(IV)-DTPA complex is more stable than the Pu(III)-DTPA complex. The equilibrium constants $(\log \mathrm{K})$ for the $\mathrm{Pu}(\mathrm{IV})$ - and $\mathrm{Pu}(\mathrm{III})$-DTPA complexes are 35.39 and 29.5, respectively.[11] 
The actinide distribution data are not a strong function of temperature over the range studied. The Np(IV) distribution coefficients decrease with temperature while the $\mathrm{Pu}(\mathrm{IV}), \mathrm{Am}(\mathrm{III})$, and $\mathrm{Pa}$ (V) are nearly constant within the accuracy of the measurements. A decrease in the distribution coefficients with temperature is due to the exothermic nature of the extraction process. Conditional enthalpies of extraction determined by van't Hoff analysis are calculated in the following section using the distribution data. The uncertainty in the distribution coefficients generally increases at the high and low temperatures used for the extractions. This is likely due to the increased temperature gradient in the Thermomixer $\mathrm{R}$ heating/cooling block. Below $20^{\circ} \mathrm{C}$ and above $45^{\circ} \mathrm{C}$, the uncertainty in the temperature control increases from $\pm 0.5^{\circ} \mathrm{C}$ to $\pm 2{ }^{\circ} \mathrm{C}$.

\section{van't Hoff Analysis}

The actinide distribution coefficients measured at different temperatures can be used to calculate conditional enthalpies $\left(\Delta \mathrm{H}^{0}\right)$ and entropies $\left(\Delta \mathrm{S}^{0}\right)$ of extraction. The thermodynamic properties are considered conditional, since they are based on the variation of distribution coefficients as a function of temperature, rather than the extraction equilibrium constant $(\mathrm{K})$.[7]

The free energy of extraction is defined by equations 5 and 6 .

$$
\begin{gathered}
\Delta \mathrm{G}^{0}=\Delta \mathrm{H}^{0}-\mathrm{T} \Delta \mathrm{S}^{0} \\
\Delta \mathrm{G}^{0}=-\mathrm{RT} \ln \mathrm{K}
\end{gathered}
$$

Combining the equations and rearrangement results in equation 7 ,

$$
\ln \mathrm{K}=-\frac{\Delta \mathrm{H}^{0}}{\mathrm{RT}}+\frac{\Delta \mathrm{S}^{0}}{\mathrm{R}}
$$

which shows that a plot of the natural log of the extraction equilibrium constant as a function of the reciprocal thermodynamic temperature should be linear with a slope of $-\Delta H^{0} / R$ and a y-intercept of $\Delta S^{0} / R$. To calculate the conditional enthalpies and entropies of extraction for the TALSPEAK process, the natural $\log$ of the actinide distribution coefficients were plotted as a function of the reciprocal thermodynamic extraction temperature (see Figures 12-15 for $\mathrm{Np}(\mathrm{IV}), \mathrm{Pu}(\mathrm{IV}), \mathrm{Am}(\mathrm{III})$, and $\mathrm{Pa}(\mathrm{V})$, respectively) and linear regressions were performed. The thermodynamic properties calculated from the

\begin{tabular}{|c|c|c|c|c|c|c|}
\hline Element & $\begin{array}{c}\Delta \mathrm{H}^{0} \\
(\mathrm{~kJ} / \mathrm{mol})\end{array}$ & $\begin{array}{c}\text { Std. Dev. } \\
\Delta \mathrm{H}^{0} \\
(\mathrm{~kJ} / \mathrm{mol})\end{array}$ & $\begin{array}{c}\text { Rel. Std. Dev. } \\
\Delta \mathrm{H}^{0} \\
(\%)\end{array}$ & $\begin{array}{c}\Delta \mathrm{S}^{0} \\
(\mathrm{~J} / \mathrm{mol} \mathrm{K})\end{array}$ & $\begin{array}{c}\text { Std. Dev. } \\
\Delta \mathrm{S}^{0} \\
(\mathrm{~J} / \mathrm{mol} \mathrm{K})\end{array}$ & $\begin{array}{c}\text { Rel. Std. Dev. } \\
\Delta \mathrm{S}^{0} \\
(\%)\end{array}$ \\
\hline$N p(I V)$ & -12.5 & 3.3 & 26.3 & -81.9 & 11 & 13.4 \\
\hline $\mathrm{Pu}(\mathrm{IV})$ & 1.3 & 1.1 & 81.9 & -25.6 & 3.7 & 14.4 \\
\hline
\end{tabular}
van't Hoff analysis are provided in Table 9 (see Appendix D for calculations).

Table 9 Thermodynamic properties calculated by van't Hoff analysis

The correlations of the Am and Pa data were so poor that thermodynamic properties were not calculated. The uncertainty in the conditional enthalpies of extraction for $\mathrm{Np}$ and $\mathrm{Pu}$ were also larger than desired. The poor correlation of the distribution data can likely be attributed to the low concentration of HDEHP which was used to perform the extractions (i.e., only $0.17 \mathrm{M}$ ). Since tracer level concentrations of ${ }^{238} \mathrm{Pu}$ and ${ }^{241} \mathrm{Am}$ (and only 3-4 g/L ${ }^{237} \mathrm{~Np}$ ) were utilized in the experiments, the total counts extracted into the organic phase were low. Based on the distribution coefficients in Table 4, the estimated total counts of Am in the organic phase was only about $1000 \mathrm{dpm} / \mathrm{mL}$. To quantify the activity levels of ${ }^{241} \mathrm{Am}$ and ${ }^{238} \mathrm{Pu}$, it was necessary to use a low level detector to count the samples. Reducing the uncertainty in the distribution data would require the use of higher actinide activities in the extraction experiments and/or a 
higher concentration of solvent. (The use of higher activities in the extractions was precluded by the total activity which can be handled in a radiochemical hood by safety procedure restrictions.) Therefore, future measurements should be performed in a glovebox where higher concentrations of the actinides can be used. The use of concentrations which are representative of those expected in the TALSPEAK process is also recommended which would eliminate concerns that extractions performed at tracer levels may not completely represent the results obtained at higher concentrations.

A summary of the conditional enthalpies and entropies of extraction for $\mathrm{Np}(\mathrm{IV}), \mathrm{Np}(\mathrm{V}), \mathrm{Pu}(\mathrm{III}), \mathrm{Pu}(\mathrm{IV})$, $\mathrm{Am}(\mathrm{III})$, and $\mathrm{Pa}(\mathrm{V})$ is given in Table 10. These data include experimentally measured values from this work and a previous study.[3]

Table 10 Conditional enthalpies and entropies of extractions for actinide elements

\begin{tabular}{cccccccc}
\hline \hline Element & $\Delta \mathrm{H}^{0}$ & $\begin{array}{c}\text { Std. Dev. } \\
\Delta \mathrm{H}^{0} \\
(\mathrm{~kJ} / \mathrm{mol})\end{array}$ & $\begin{array}{c}\Delta \mathrm{H}^{0} \\
(\%)\end{array}$ & $\begin{array}{c}\text { Rel. Std. Dev. } \\
(\mathrm{kJ} / \mathrm{mol})\end{array}$ & $\begin{array}{c}\Delta \mathrm{S}^{0} \\
\Delta \mathrm{S}^{0} \\
(\mathrm{~J} / \mathrm{mol} \mathrm{K})\end{array}$ & $\begin{array}{c}\text { Std. Dev. Std. Dev. } \\
\Delta \mathrm{S}^{0}\end{array}$ & $\begin{array}{c}\text { Ref. } \\
(\%)\end{array}$ \\
\hline $\mathrm{Np}(\mathrm{IV})$ & -12.5 & 3.3 & 26.3 & -81.9 & 11 & 13.4 & \\
$\mathrm{~Np}(\mathrm{~V})$ & -39.6 & 4.9 & 12.3 & -122 & 16 & 12.9 & {$[3]$} \\
$\mathrm{Pu}(\mathrm{III})$ & -10.5 & 5.0 & 47.4 & -60.4 & 16.0 & 26.6 & {$[3]$} \\
$\mathrm{Pu}(\mathrm{IV})$ & 1.3 & 1.1 & 81.9 & -25.6 & 3.7 & 14.4 & \\
$\mathrm{Am}(\mathrm{III})$ & -10.7 & 1.9 & 17.5 & -41.6 & 6.1 & 14.6 & {$[3]$} \\
$\mathrm{Pa}(\mathrm{V})$ & -48.3 & 9.0 & 18.7 & -125 & 29 & 23.3 & {$[3]$} \\
\hline
\end{tabular}

The data from reference 3 were obtained using an aqueous phase which consisted of a $1.5 \mathrm{M}$ lactic acid/ammonium lactate buffer containing $0.05 \mathrm{M} \mathrm{DTPA}$ at $\mathrm{pH} 3.5$ and an organic phase containing $1 \mathrm{M}$ HDEHP. The temperature range of the experiments was 20 to $60^{\circ} \mathrm{C}$. One interesting observation from Table 10 is the endothermic nature of the Pu(IV) extraction. Although, due to the large uncertainty in the calculation, it is possible that the extraction of Pu(IV) into HDEHP could be slightly exothermic.

\section{CONCLUSIONS}

To better understand the stability of $\mathrm{Pu}(\mathrm{III})$ and $\mathrm{Np}(\mathrm{V})$ in the TALSPEAK solvent extraction process, an experimental study was performed to measure the oxidation/reduction kinetics in a $1 \mathrm{M}$ buffered lactate solution containing $0.05 \mathrm{M}$ DTPA. Time-dependent UV-vis spectra were used to calculate first order rate constants for the oxidation of $\mathrm{Pu}$ (III) to $\mathrm{Pu}$ (IV) and the reduction of $\mathrm{Np}$ (V) to $\mathrm{Np}$ (IV). The stability of $\mathrm{Pu}$ (IV)-DTPA and Np(IV)-DTPA complexes are much greater than the stability of the Pu(III)-DTPA and $\mathrm{Np}(\mathrm{V})$-DTPA complexes, and as a result, $\mathrm{Pu}$ is slowly oxidized to $\mathrm{Pu}(\mathrm{IV})$ and $\mathrm{Np}$ is slowly reduced to $\mathrm{Np}$ (IV) due to the reduced activity of the stable complexes. The rate constants for the oxidation of Pu(III) and reduction of $\mathrm{Np}(\mathrm{V})$ were calculated from changes in absorbance at peaks in the spectra which are characteristic of $\mathrm{Pu}(\mathrm{III})$-DTPA and $\mathrm{Np}(\mathrm{V})$-DTPA complexes. The oxidation/reduction rate constants are specific to the $\mathrm{Pu}(\mathrm{III})$ and $\mathrm{Np}(\mathrm{V})$-DTPA complexes which absorb at these wavelengths. The timedependent spectra for both elements had four to five isosbestic points which indicate the presence of multiple Pu and $\mathrm{Np}$ species in the lactic acid/DTPA solution. Additional experiments and analysis would be required to develop speciation diagrams that would allow the calculation of the actinide-DTPA concentrations as functions of time. The rate constants calculated for $\mathrm{Pu}$ resulted in half-times for the oxidation of $\mathrm{Pu}(\mathrm{III})$ between approximately 8 and $20 \mathrm{~h}$. The half-times for the reduction of $\mathrm{Np}(\mathrm{V})$ were 17 and $171 \mathrm{~h}$. The difference in magnitude of the $\mathrm{Np}$ reduction rates was attributed to the difference in the stability of the $\mathrm{Np}(\mathrm{V})$-DTPA complexes which absorbed at the two wavelengths of interest.

To examine the thermodynamic driving force for the extraction of actinide elements in the TALSPEAK process, an experimental study was performed to measure the distribution of $\mathrm{Np}$ (IV), $\mathrm{Pu}$ (IV), $\mathrm{Am}$ (III), and $\mathrm{Pa}(\mathrm{V})$ between the aqueous and organic phases as a function of temperature. We found that Am(III) 
and especially $\mathrm{Pa}(\mathrm{V})$ were more strongly extracted into the HDEHP solvent than $\mathrm{Np}(\mathrm{IV})$ or $\mathrm{Pu}(\mathrm{IV})$. The decreased extent of extraction for the 4+ actinides was attributed to the stability of the An(IV)-DTPA complexes (compared to either the $5+$ or $3+$ complexes) which limits their extraction into the HDEHP solvent. The actinide distribution data were not a strong function of temperature. The Np(IV) distribution coefficients decreased with temperature while the $\mathrm{Pu}(\mathrm{IV}), \mathrm{Am}(\mathrm{III})$, and $\mathrm{Pa}$ (V) were nearly invariant over the range studied (and within the measurement uncertainty). Conditional enthalpies and entropies of extraction for $\mathrm{Np}(\mathrm{IV})$ and $\mathrm{Pu}$ (IV) were calculated by van't Hoff analysis. The correlation of the distribution data for $\mathrm{Am}$ (III) and $\mathrm{Pa}(\mathrm{IV})$ was poor and thermodynamic properties could not be calculated. The poor correlation of the distribution data was attributed to the low concentration of HDEHP used in the experiment which resulted in an insufficient amount of activity in the organic phase to obtain consistent results. The conditional enthalpies of extraction for $\mathrm{Np}$ (IV) and $\mathrm{Pu}(\mathrm{IV})$ were more positive than values previously measured for $\mathrm{Np}(\mathrm{V})$ and $\mathrm{Pu}(\mathrm{III})$ which is consistent with more stable DTPA complexes limiting the extraction of $4+$ actinides.

\section{RECOMMENDATIONS FOR FUTURE WORK}

The time-dependent spectra for the oxidation of $\mathrm{Pu}$ (III) to $\mathrm{Pu}$ (IV) and the reduction of $\mathrm{Np}$ (V) to $\mathrm{Np}$ (IV) indicate that multiple $\mathrm{Pu}$ and $\mathrm{Np}$ complexes are present in the lactic acid/DTPA solutions. Additional experiments and analysis should be performed to develop speciation diagrams that would allow a more complete interpretation of the UV-vis spectroscopy which was observed. Reducing the uncertainty in the distribution data will require the use of higher actinide activities in the extraction experiments or a higher concentration of solvent. Since the use of higher activities with the existing methods is limited by the total activity which can be handled in a radiochemical hood, we recommend performing the distribution coefficient measurements in a glovebox where higher concentrations of the actinides can be safely used. The use of actinide concentrations representative of those expected in the TALSPEAK process is also recommended which would eliminate concerns that extractions performed at tracer levels may not completely represent the results obtained at higher concentrations.

\section{REFERENCES}

1. Nuclear Energy Research and Development Roadmap - Report to Congress, United States Department of Energy Office of Nuclear Energy, Washington, DC (2010).

2. L. R. Martin and P. R. Zalupski, Thermodynamics and Kinetics of Advanced Separations Systems FY2010 Summary Report, Report FCR\&D-SEPA-2010-000174, Idaho National Laboratory, Idaho Falls, ID (September 2010).

3. T. S. Rudisill and E. A. Kyser III, Distribution of Actinides between the Aqueous and Organic Phases in the TALSPEAK Process, Report FCRD-SEPA-2010-000136 (SRNL-STI-2010-00502), Savannah River National Laboratory, Aiken, SC (September 2010).

4. L. R. Morss, N. M. Edelstein, and J. Fuger, Eds., The Chemistry of the Actinide and Transactinide Elements, $3^{\text {rd }}$ ed., Springer, Dordrecht, The Netherlands, Vol. 2, pp 841-844 (2006).

5. L. R. Morss et al., pp 704-705.

6. M. L. Hyder, W. C. Perkins, M. C. Thompson, G. A. Burney, E. R. Russell, H. P. Holcomb, and L. F. Landon, Processing of Irradiated Enriched Uranium Fuels at the Savannah River Plant, DP-1500, E. I. du Pont de Nemours \& Co., Aiken, SC (1979).

7. M. Nilsson and K. L. Nash, Review Article: A Review of the Development and Operational Characteristics of the TALSPEAK Process, Solvent Extr Ion Exc, 25, pp 665-701 (2007).

8. B. Weaver and F. A. Kappelmann, Talspeak, A new method of separating americium and curium from the lanthanides by extraction from an aqueous solution of an aminopolyacetic acid complex with 
a monoacetic organophosphate or phosphonate, ORNL-3559, Union Carbide Corporation, Oak Ridge, TN (1964).

9. H. H. Willard, L. L. Merrit, Jr., J. A. Dean, and F. A. Settle, Jr., Instrumental Methods of Analysis, $6^{\text {th }}$ Ed., Wadsworth Publishing Company, Belmont, CA p. 70 (1981).

10. L. R. Morss et al., p 780.

11. L. R. Morss et al., p 1178.

12. J. M. Cleveland, The Chemistry of Plutonium, American Nuclear Society, La Grange Park, IL, p 12 (1979).

Figure 1 Comparison of $\mathrm{Pu}(\mathrm{IV})$ reference spectrum and initial $\mathrm{Pu}(\mathrm{III})$ spectrum from Experiment 1

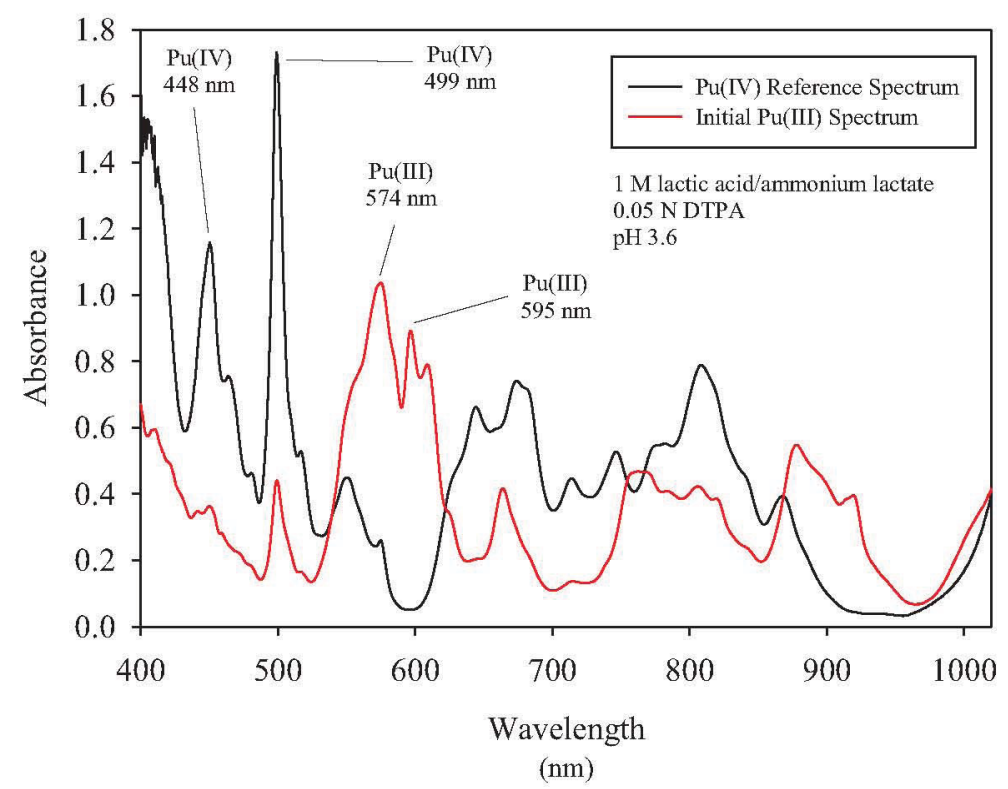

Figure 2 Comparison of $\mathrm{Pu}(\mathrm{IV})$ reference spectrum and initial $\mathrm{Pu}(\mathrm{III})$ spectrum from Experiment 2

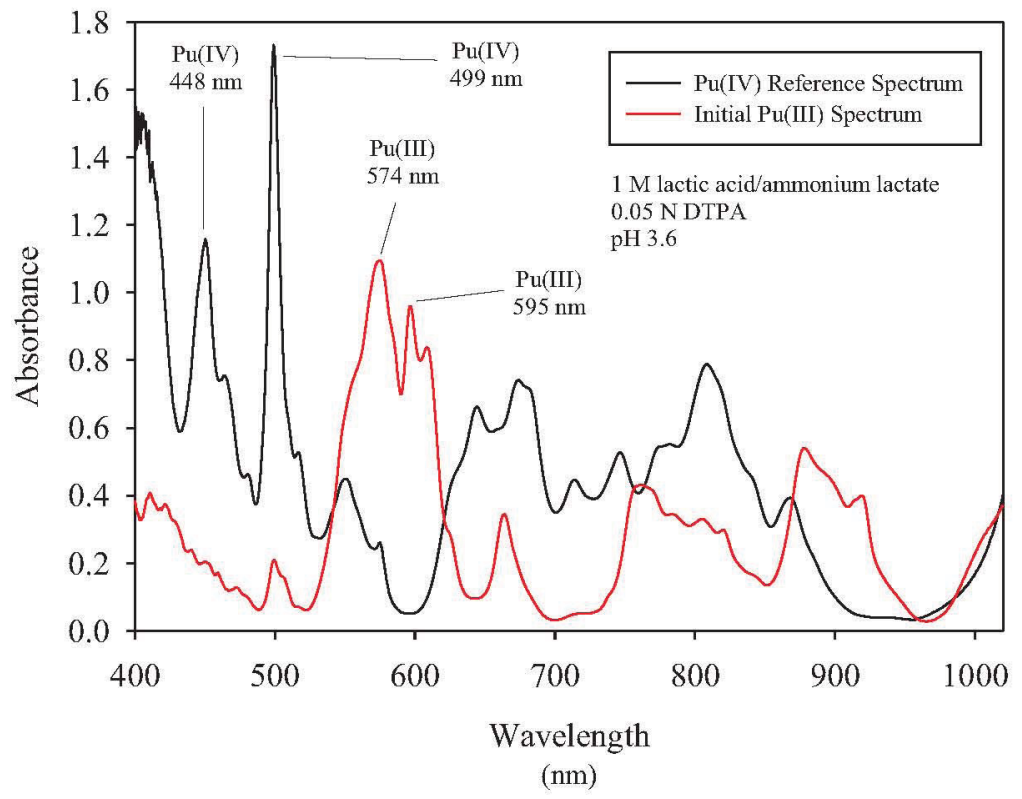


Figure 3 Absorbance at selected wavelengths during the oxidation of $\mathrm{Pu}$ (III) to $\mathrm{Pu}(\mathrm{IV})$

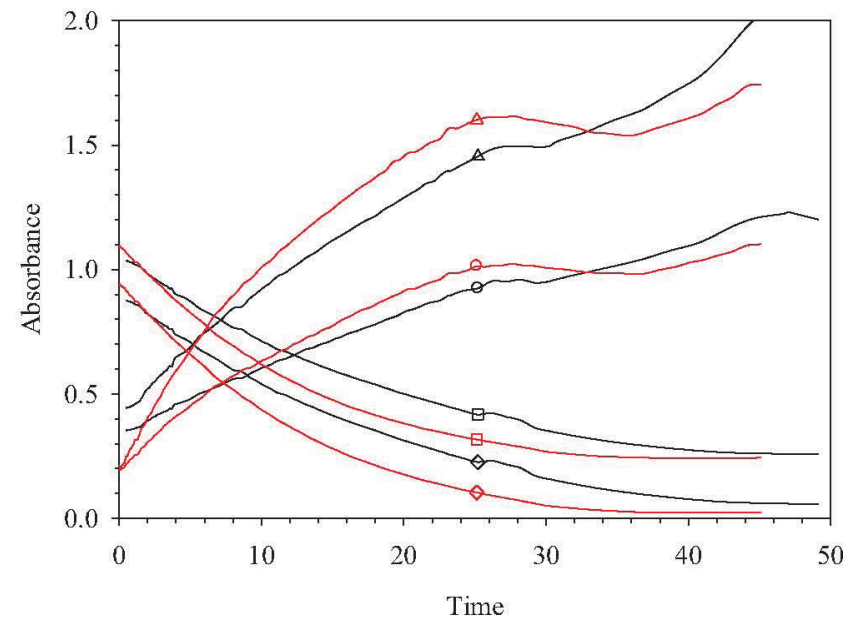

(h)

$$
\begin{array}{ll}
\circ & 448 \mathrm{~nm} \text { Pu(IV) - Exp } 1 \\
\Delta & 499 \mathrm{~nm} \text { Pu(IV) - Exp 1 } \\
\square & 574 \mathrm{~nm} \text { Pu(III) - Exp 1 } \\
\diamond & 595 \mathrm{~nm} \text { Pu(III) - Exp 1 } \\
0 & 448 \mathrm{~nm} \text { Pu(IV) - Exp 2 } \\
\Delta & 499 \mathrm{~nm} \text { Pu(IV) - Exp 2 } \\
\square & 574 \mathrm{~nm} \text { Pu(III) - Exp 2 } \\
\diamond & 595 \mathrm{~nm} \mathrm{Pu} \text { (III) - Exp 2 }
\end{array}
$$

Figure 4 UV-vis spectra for oxidation of $\mathrm{Pu}(\mathrm{III})$ to $\mathrm{Pu}(\mathrm{IV})$ - Experiment 1

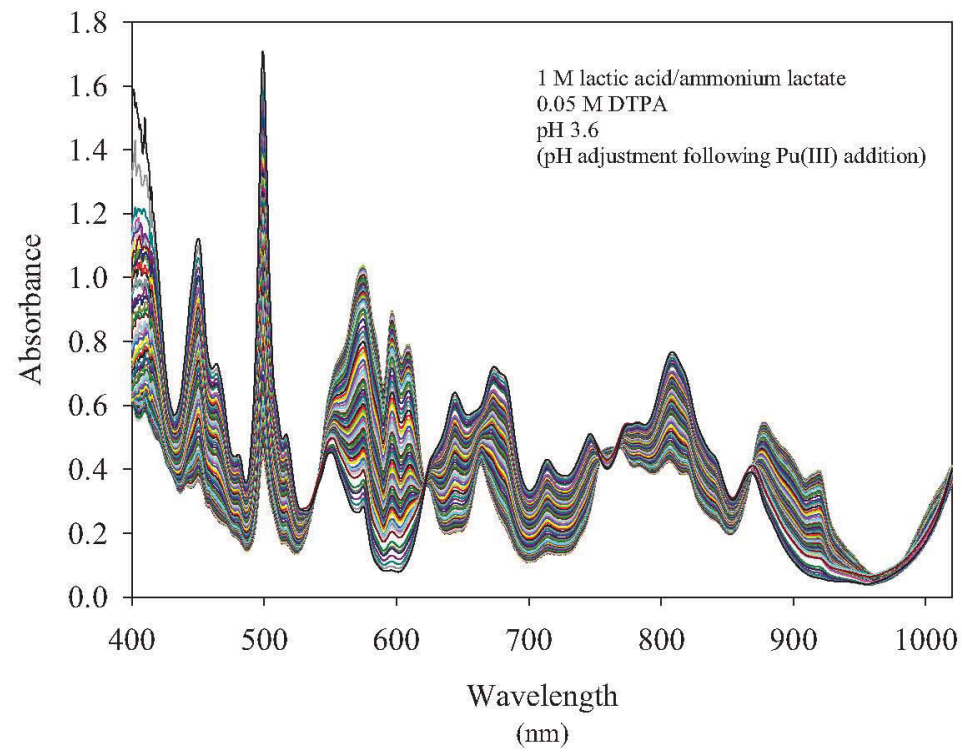


Figure 5 UV-vis spectra for oxidation of $\mathrm{Pu}(\mathrm{III})$ to $\mathrm{Pu}(\mathrm{IV})$ - Experiment 2

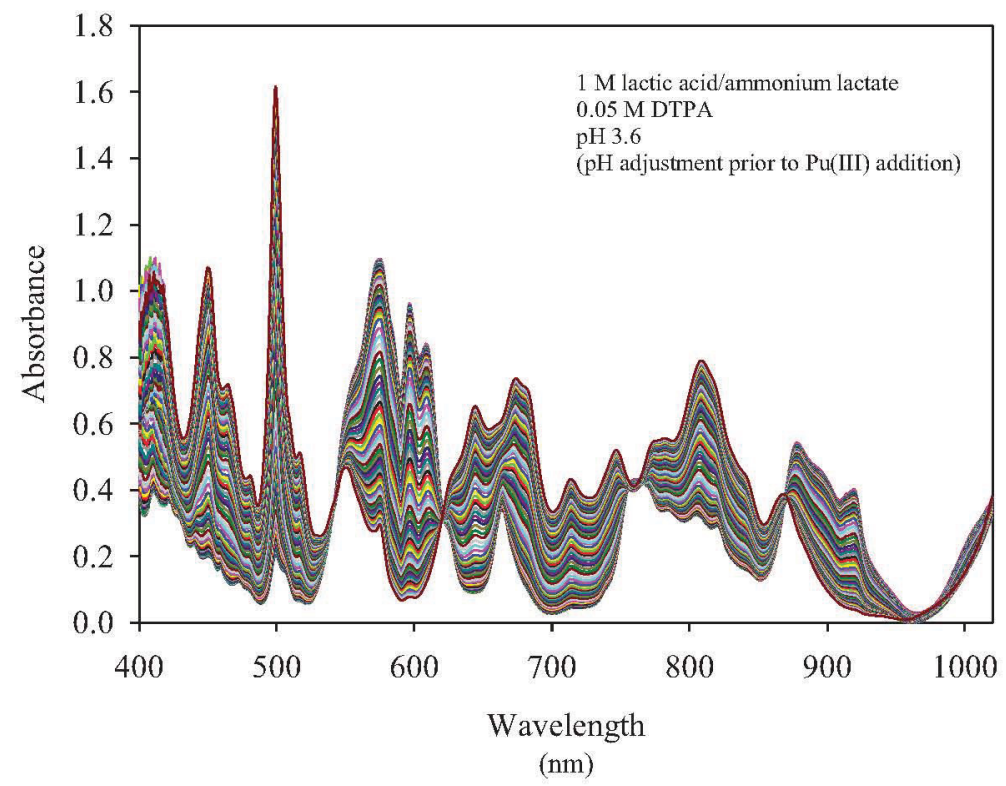

Figure 6 Calculation of rate constants for the oxidation of Pu(III) - Experiment 1

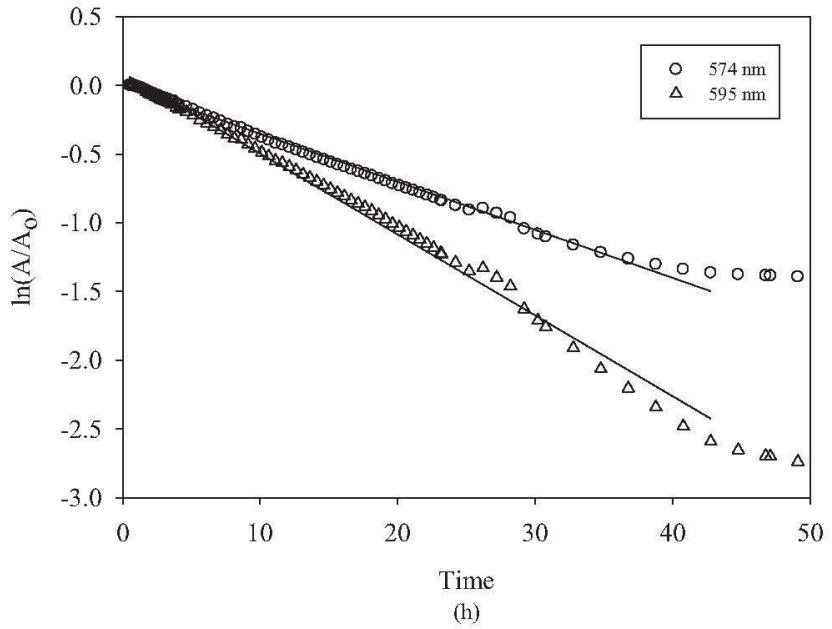


Figure 7 Calculation of rate constants for the oxidation of $\mathrm{Pu}(\mathrm{III})$ - Experiment 2

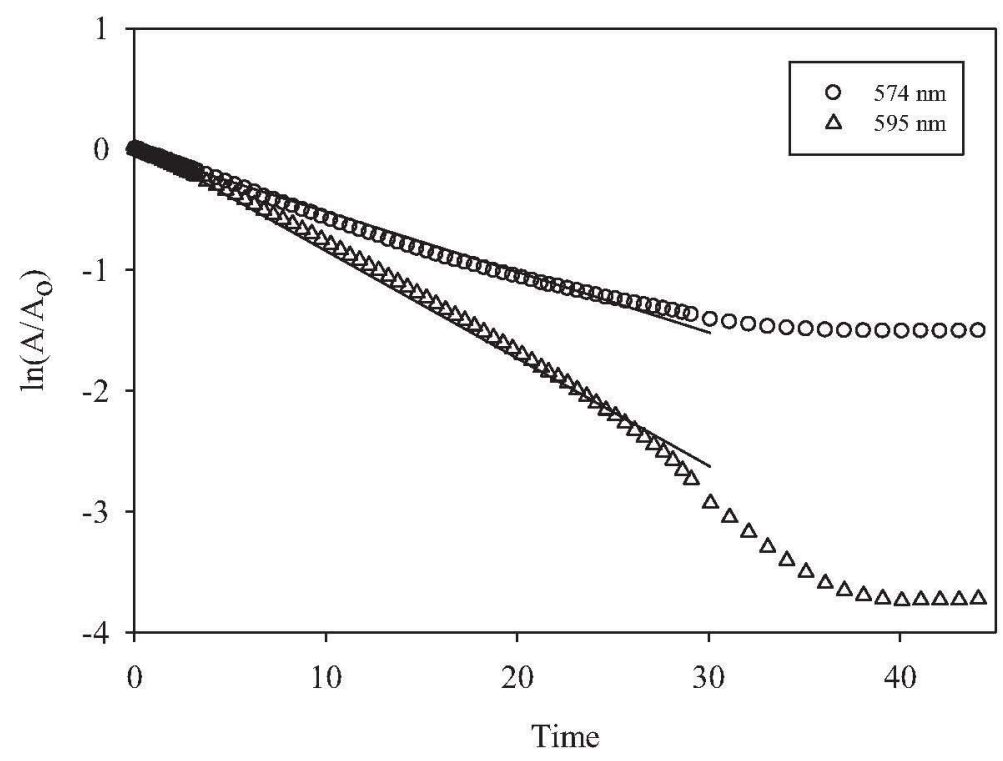

(h)

Figure $8 \mathrm{UV}$-vis reference spectra for $\mathrm{Np}(\mathrm{V})$ reduction

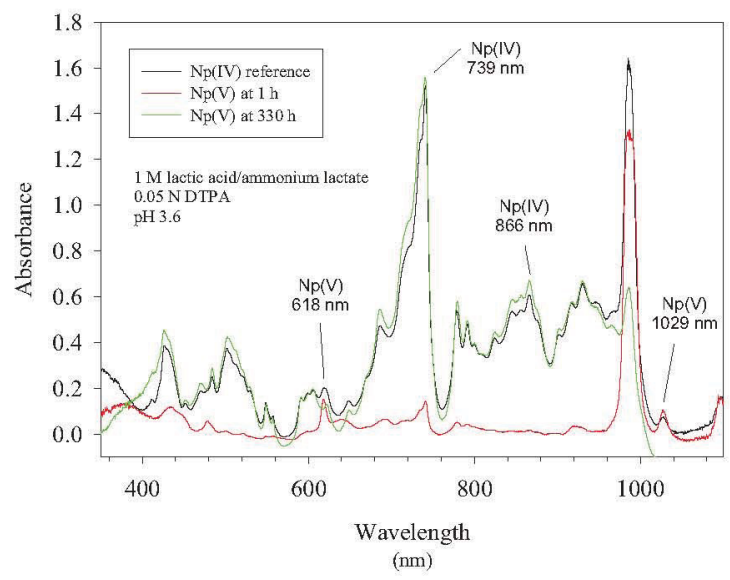


Figure 9 Absorbance at selected wavelengths in $\mathrm{Np}(\mathrm{V}) \mathrm{UV}$-vis spectra

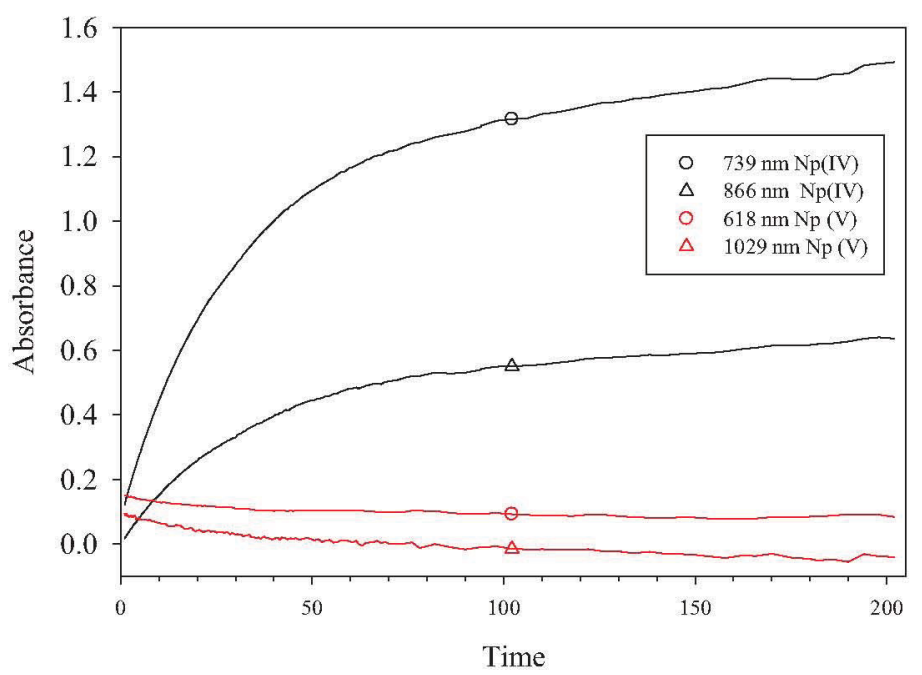

(h)

Figure $10 \mathrm{UV}$-vis spectra for reduction of $\mathrm{Np}(\mathrm{V})$ to $\mathrm{Np}(\mathrm{IV})$

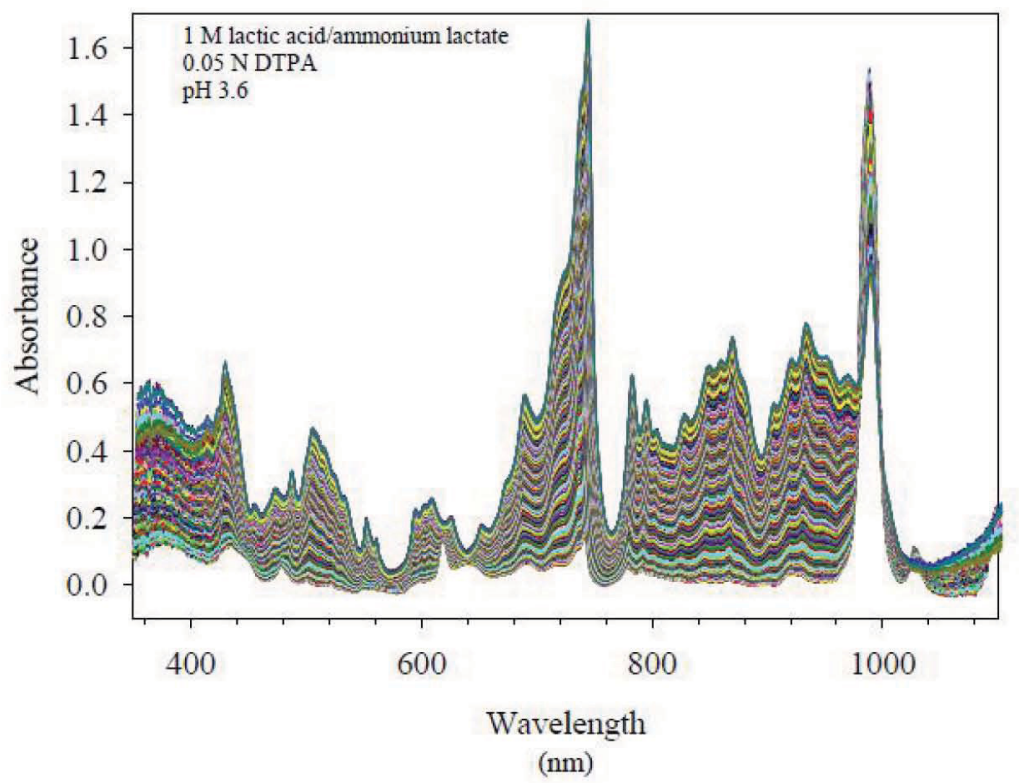


Figure 11 Calculation of rate constants for the reduction of $\mathrm{Np}(\mathrm{V})$

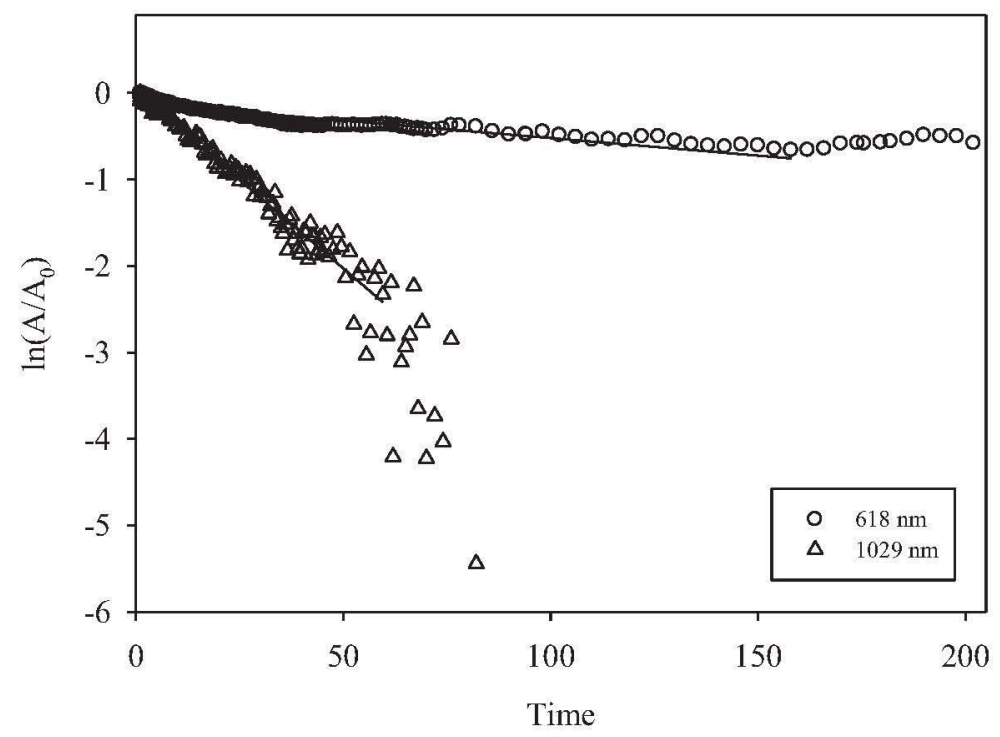

(h)

Figure 12 van’t Hoff analysis for $\mathrm{Np}$ extraction

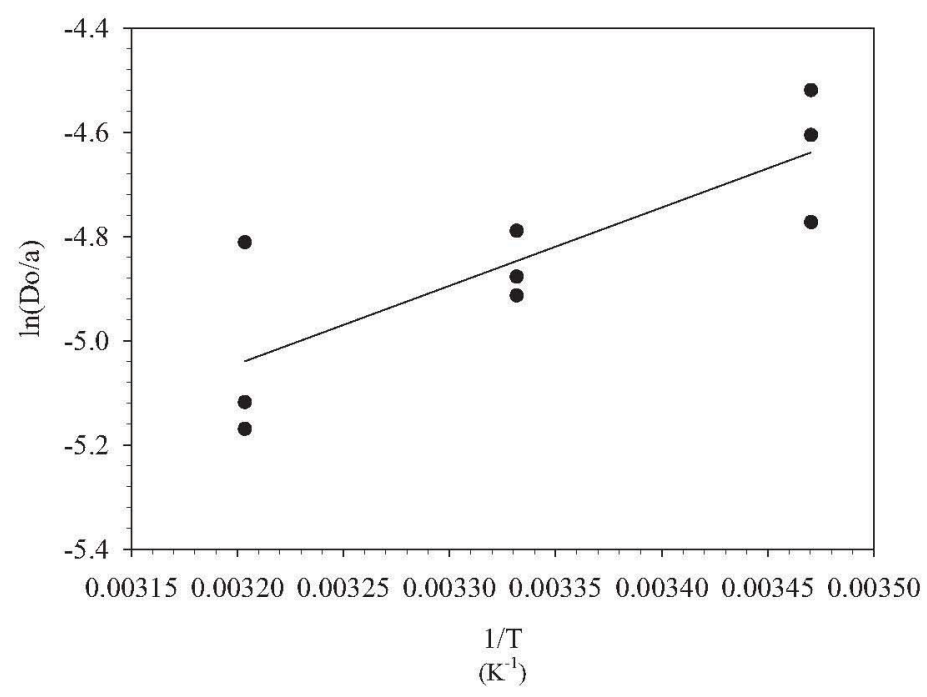


Figure 13 van't Hoff analysis for Pu extraction

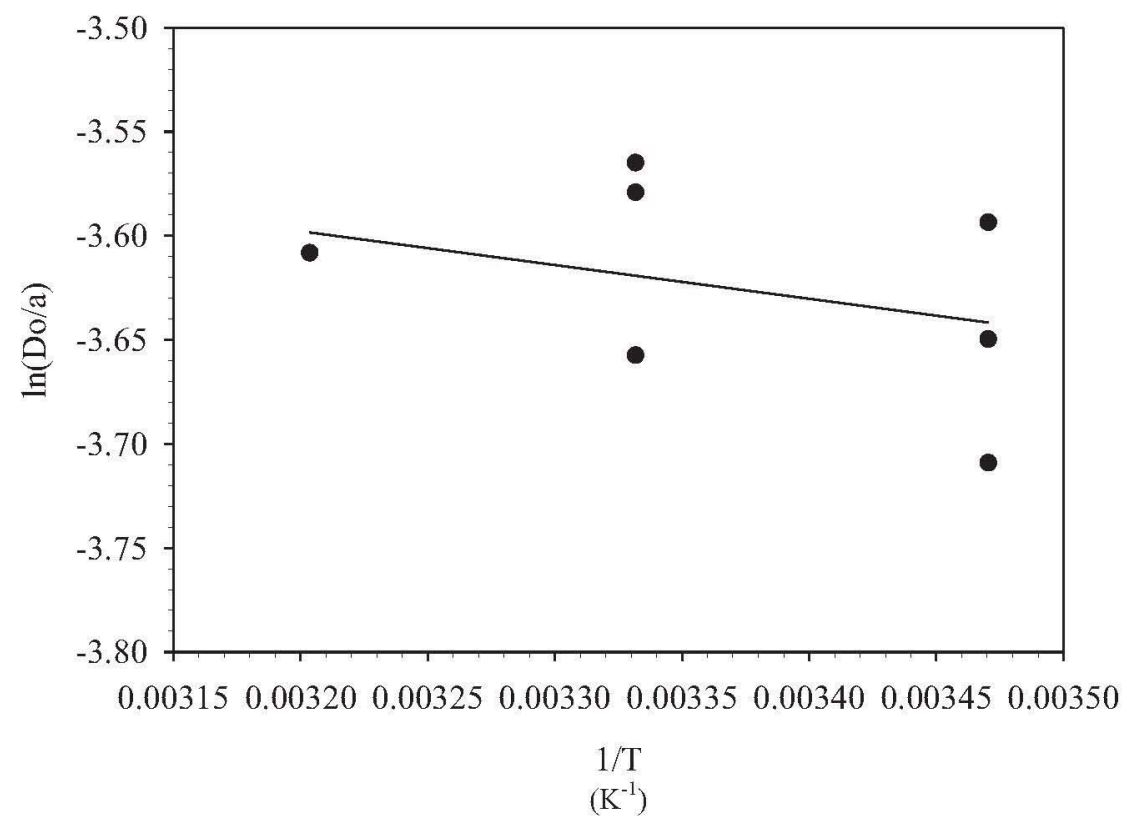

Figure 14 van't Hoff analysis for Am extraction

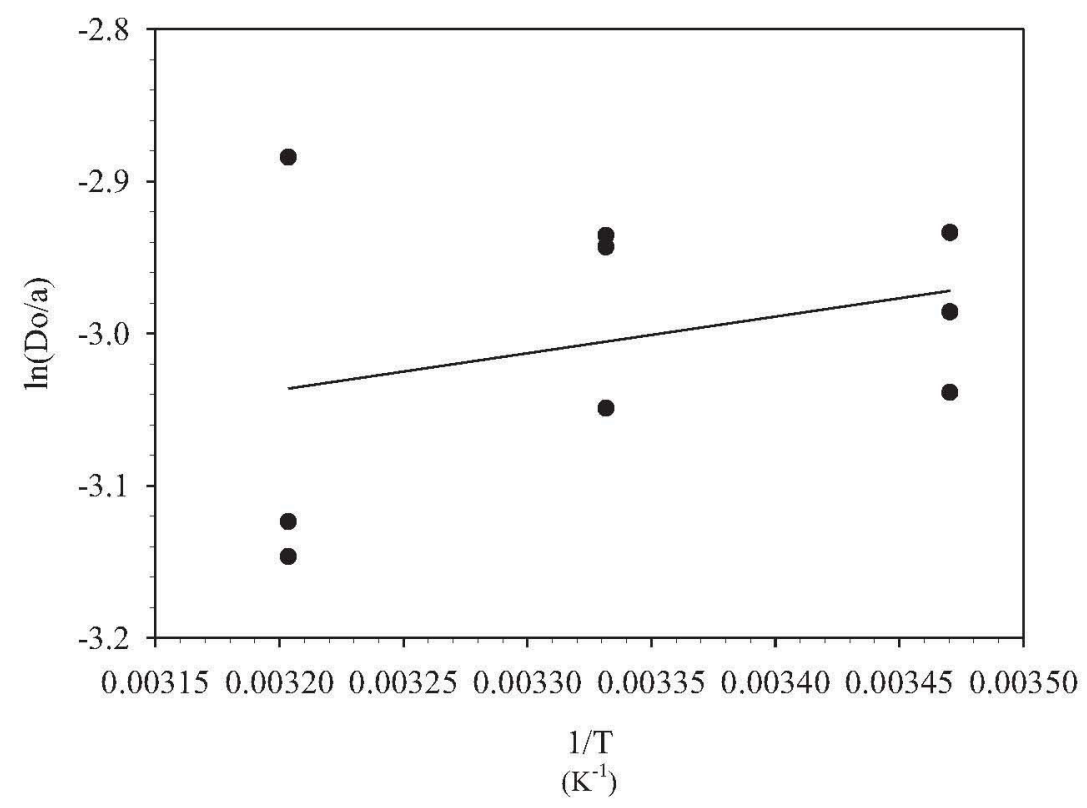


Figure 15 van't Hoff analysis for Pa extraction

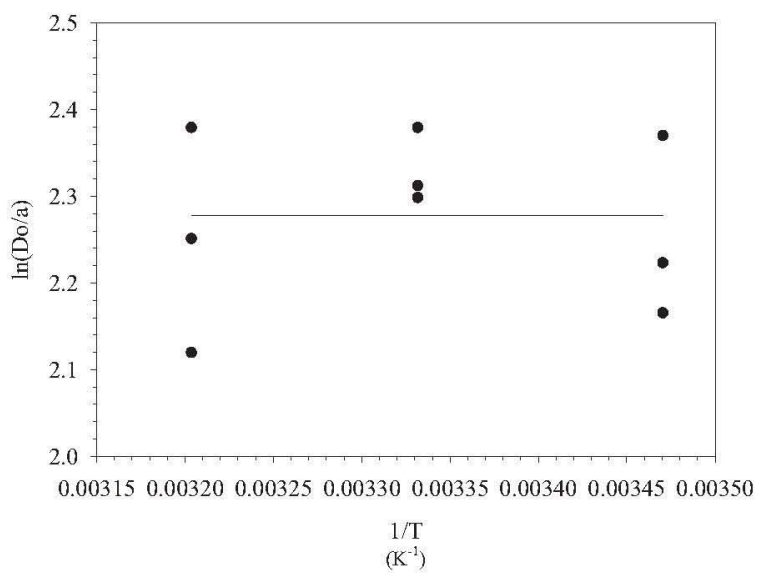

